\title{
Exotic symmetric spaces of higher level - Springer correspondence for complex reflection groups -
}

\author{
Toshiaki Shoji
}

\begin{abstract}
Let $G=G L(V)$ for a $2 n$-dimensional vector space $V$, and $\theta$ an involutive automorphism of $G$ such that $H=G^{\theta} \simeq S p(V)$. Let $G_{\text {uni }}^{\iota \theta}$ be the set of unipotent elements $g \in G$ such that $\theta(g)=g^{-1}$. For any integer $r \geq 2$, we consider the variety $G_{\text {uni }}^{\iota \theta} \times V^{r-1}$, on which $H$ acts diagonally. Let $W_{n, r}=S_{n} \ltimes(\mathbf{Z} / r \mathbf{Z})^{n}$ be a complex reflection group. In this paper, generalizing the known result for $r=2$, we show that there exists a natural bijective correspondence (Springer correspondence) between the set of irreducible representations of $W_{n, r}$ and a certain set of $H$-equivariant simple perverse sheaves on $G_{\text {uni }}^{\iota \theta} \times V^{r-1}$. We also consider a similar problem for $G \times V^{r-1}$, on which $G$ acts diagonally, where $G=G L(V)$ for a finite dimensional vector space $V$.
\end{abstract}

\section{INTRODUCTION}

Let $V$ be a $2 n$ dimensional vector space over $\mathbf{k}$, where $\mathbf{k}$ is an algebraically closed field with char $\mathbf{k} \neq 2$. Let $G=G L(V)$ and $\theta: G \rightarrow G$ an involutive automorphism such that $G^{\theta} \simeq S p(V)$. Let $\iota: G \rightarrow G$ be the anti-automorphism $g \mapsto g^{-1}$, and put $G^{\iota \theta}=\left\{g \in G \mid \theta(g)=g^{-1}\right\}$. We consider the variety $\mathcal{X}=G^{\iota \theta} \times V$, on which $H=G^{\theta}$ acts diagonally. Let $G_{\text {uni }}^{\iota \theta}$ be the set of unipotent elements in $G^{\iota \theta}$, and define a closed subvariety $\mathcal{X}_{\text {uni }}$ of $\mathcal{X}$ by $\mathcal{X}_{\text {uni }}=G_{\text {uni }}^{\iota \theta} \times V$. $\mathcal{X}_{\text {uni }}$ is nothing but the exotic nilpotent cone introduced by Kato $[\mathrm{K}]$. It is known that $\mathcal{X}_{\text {uni }}$ is $H$-stable, and the set of $H$-orbits in $\mathcal{X}_{\text {uni }}$ is in bijection with the set $\mathcal{P}_{n, 2}$ of double partitions of $n$ $([\mathrm{K}])$. Let $B$ be a $\theta$-stabel Borel subgroup of $G, U$ the unipotent radical of $B$, and $\left(M_{i}\right)_{1 \leq i \leq n}$ be an isotropic flag in $V$ such that whose stabilizer in $H$ is $B^{\theta}$. We define a variety $\widetilde{\mathcal{X}}_{\text {uni }}$ by

$$
\widetilde{\mathcal{X}}_{\text {uni }}=\left\{\left(x, v, g B^{\theta}\right) \in G_{\text {uni }}^{\iota \theta} \times V \times H / B^{\theta} \mid g^{-1} x g \in U^{\iota \theta}, g^{-1} v \in M_{n}\right\},
$$

and define a map $\pi_{1}: \widetilde{\mathcal{X}}_{\text {uni }} \rightarrow \mathcal{X}_{\text {uni }}$ by the projection on the first two factors. In [K], [SS1], the Springer correspondence between the set of $H$-orbits in $\mathcal{X}_{\text {uni }}$ and the set of irreducible representations of the Weyl group $W_{n, 2}$ of type $C_{n}$ was studied. More precisely, it is stated as follows; $\left(\pi_{1}\right) ! \overline{\mathbf{Q}}_{l}\left[\operatorname{dim} \mathcal{X}_{\text {uni }}\right]$ is a semisimple perverse sheaf on $\mathcal{X}_{\text {uni }}$, equipped with $W_{n, 2^{-}}$action and is decomposed as

$$
\left(\pi_{1}\right)_{!} \overline{\mathbf{Q}}_{l}\left[\operatorname{dim} \mathcal{X}_{\text {uni }}\right] \simeq \bigoplus_{\boldsymbol{\lambda} \in \mathcal{P}_{n, 2}} V(\boldsymbol{\lambda}) \otimes \operatorname{IC}\left(\overline{\mathcal{O}}_{\boldsymbol{\lambda}}, \overline{\mathbf{Q}}_{l}\right)\left[\operatorname{dim} \mathcal{O}_{\boldsymbol{\lambda}}\right],
$$

where $V(\boldsymbol{\lambda})$ is the irreducible representation of $W_{n, 2}$ and $\mathcal{O}_{\boldsymbol{\lambda}}$ is the $H$-orbit in $\mathcal{X}_{\text {uni }}$ corresponding to $\boldsymbol{\lambda} \in \mathcal{P}_{n, 2}$. 
In this paper, we consider the variety $G^{\iota \theta} \times V^{r-1}$ for a positive integer $r \geq 2$, with the diagonal action of $H$. We call it the exotic symmetric space of level $r$. Let $W_{n, r}=$ $S_{n} \ltimes(\mathbf{Z} / r \mathbf{Z})^{n}$ be the complex reflection group $G(r, 1, n)$, where $S_{n}$ is the symmetric group of degree $n$. We will generalize the previous result to the correspondence between the set of irreducible representations of $W_{n, r}$ and a certain set of simple perverse sheaves on $G_{\text {uni }}^{\iota \theta} \times V^{r-1}$. Let $\mathcal{Q}_{n, r}$ be the set of $\mathbf{m}=\left(m_{1}, \ldots, m_{r}\right) \in \mathbf{Z}_{\geq 0}^{r}$ such that $\sum m_{i}=n$, and $\mathcal{Q}_{n, r}^{0}$ the subset of $\mathcal{Q}_{n, r}$ consisting of $\mathbf{m}$ such that $m_{r}=0$. For each $\mathbf{m} \in \mathcal{Q}_{n, r}$, we define varieties

$$
\begin{aligned}
& \widetilde{\mathcal{X}}_{\mathbf{m}, \text { uni }}=\left\{\left(x, \mathbf{v}, g B^{\theta}\right) \in G_{\text {uni }}^{\iota \theta} \times V^{r-1} \times H / B^{\theta} \mid g^{-1} x g \in U^{\iota \theta}, g^{-1} \mathbf{v} \in \prod_{i=1}^{r-1} M_{p_{i}}\right\}, \\
& \mathcal{X}_{\mathbf{m}, \text { uni }}=\bigcup_{g \in H} g\left(U^{\iota \theta} \times \prod_{i=1}^{r-1} M_{p_{i}}\right),
\end{aligned}
$$

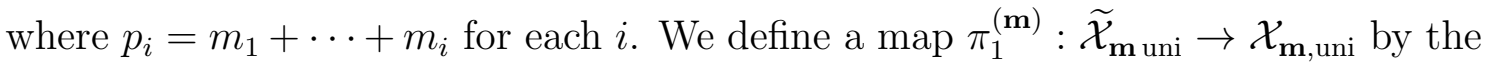
projection on the first two factors. In the case where $\mathbf{m}=(n, 0, \ldots, 0)$, we write $\widetilde{\mathcal{X}}_{\mathbf{m} \text {,uni }}, \mathcal{X}_{\mathbf{m} \text {,uni }}$ and $\pi_{1}^{(\mathbf{m})}$ simply by $\widetilde{\mathcal{X}}_{\text {uni }}, \mathcal{X}_{\text {uni }}$ and $\pi_{1}$. Note that even in this case, the map $\widetilde{\mathcal{X}}_{\text {uni }} \rightarrow G_{\text {uni }}^{\iota \theta} \times V^{r-1}$ is not surjective. For each $\mathbf{m} \in \mathcal{Q}_{n, r}$ we consider a map $\bar{\pi}_{\mathbf{m}, 1}: \pi_{1}^{-1}\left(\mathcal{X}_{\mathbf{m}, \text { uni }}\right) \rightarrow \mathcal{X}_{\mathbf{m} \text {,uni }}$. Note that $\widetilde{\mathcal{X}}_{\mathbf{m} \text {,uni }} \subset \pi_{1}^{-1}\left(\mathcal{X}_{\mathbf{m}, \text { uni }}\right) \subset \widetilde{\mathcal{X}}_{\text {uni }}$. Let $\mathcal{P}_{n, r}$ be the set of $r$-tuples of partitions $\boldsymbol{\lambda}=\left(\lambda^{(1)}, \ldots, \lambda^{(r)}\right)$ such that $\sum_{i}\left|\lambda^{(i)}\right|=n$. For $\mathbf{m} \in \mathcal{Q}_{n, r}^{0}$, let $\widetilde{\mathcal{P}}(\mathbf{m})$ be the set of all $\boldsymbol{\lambda}=\left(\lambda^{(1)}, \ldots, \lambda^{(r)}\right) \in \mathcal{P}_{n, r}$ such that $\left|\lambda^{(i)}\right|=m_{i}$ for $i=1, \ldots, r-2$ (hence $\left|\lambda^{(r-1)}\right|=k$ for $0 \leq k \leq m_{r-1}$ ). As a generalization of (1), we prove the following result, which is regarded as the Springer correspondence for $W_{n, r}$. Assume that $\mathbf{m} \in \mathcal{Q}_{n, r}^{0}$. Then the complex $\left(\bar{\pi}_{\mathbf{m}, 1}\right)_{!} \overline{\mathbf{Q}}_{l}\left[\operatorname{dim} \mathcal{X}_{\mathbf{m}, \text { uni }}\right]$ is a semisimple perverse sheaf on $\mathcal{X}_{\mathbf{m} \text {,uni }}$, equipped with a $W_{n, r}$-action, and is decomposed as

$$
\left(\bar{\pi}_{\mathbf{m}, 1}\right)_{!} \overline{\mathbf{Q}}_{l}\left[\operatorname{dim} \mathcal{X}_{\mathbf{m}, \text { uni }}\right] \simeq \bigoplus_{\boldsymbol{\lambda} \in \widetilde{\mathcal{P}}(\mathbf{m})} V(\boldsymbol{\lambda}) \otimes \operatorname{IC}\left(\bar{X}_{\boldsymbol{\lambda}}, \overline{\mathbf{Q}}_{l}\right)\left[\operatorname{dim} X_{\boldsymbol{\lambda}}\right]
$$

where $V(\boldsymbol{\lambda})$ is an irreducible representation of $W_{n, r}$, and $X_{\boldsymbol{\lambda}}$ is a certain smooth

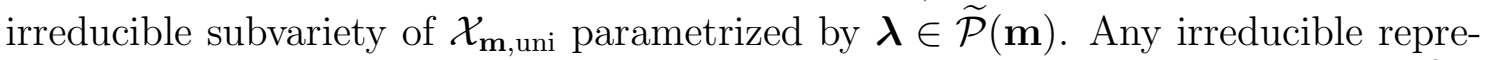
sentation of $W_{n, r}$ is realized in this way uniquely for a suitable choice of $\mathbf{m} \in \mathcal{Q}_{n, r}^{0}$. We can determine the varieties $X_{\boldsymbol{\lambda}}$ explicitly. Note that in the case $r \geq 3, G_{\text {uni }}^{\iota \theta} \times V^{r-1}$ has infinitely many $H$-orbits. So the description of $X_{\boldsymbol{\lambda}}$ becomes more complicated compared to the case where $r=2$.

In the course of the proof, we show a weaker version of the Springer correspondence. For each $\mathbf{m} \in \mathcal{Q}_{n, r}^{0}$, we define a subgroup $\mathcal{W}_{\mathbf{m}}^{\natural}$ of $W_{n, 2}$ by

$$
\mathcal{W}_{\mathbf{m}}^{\natural}=S_{m_{1}} \times \cdots \times S_{m_{r-2}} \times W_{m_{r-1}, 2} .
$$


For $\boldsymbol{\lambda} \in \widetilde{\mathcal{P}}(\mathbf{m})$, one can associate an irreducible representation $\rho_{\boldsymbol{\lambda}}$ of $\mathcal{W}_{\mathbf{m}}^{\natural}$ in a canonical way. We show that the complex $\left(\pi_{1}^{(\mathbf{m})}\right)_{!} \overline{\mathbf{Q}}_{l}\left[\operatorname{dim} \mathcal{X}_{\mathbf{m} \text {,uni }}\right]$ is a semisimple perverse sheaf on $\mathcal{X}_{\mathbf{m} \text { uni }}$, equipped with $\mathcal{W}_{\mathbf{m}}^{\natural}$-action, and is decomposed as

$$
\left(\pi_{1}^{(\mathbf{m})}\right)_{!} \overline{\mathbf{Q}}_{l}\left[\operatorname{dim} \mathcal{X}_{\mathbf{m}, \text { uni }}\right] \simeq \underset{\boldsymbol{\lambda} \in \widetilde{\mathcal{P}}(\mathbf{m})}{\bigoplus} \rho_{\boldsymbol{\lambda}} \otimes \operatorname{IC}\left(\bar{X}_{\boldsymbol{\lambda}}, \overline{\mathbf{Q}}_{l}\right)\left[\operatorname{dim} X_{\boldsymbol{\lambda}}\right]
$$

Any irreducible representation of $\mathcal{W}_{\mathbf{m}}^{\natural}$ is realized in this way uniquely for a suitable choice of $\mathbf{m} \in \mathcal{Q}_{n, r}^{0}$. Note that the group $W_{n, r}$ is not directly relatd to the geometry of $H / B^{\theta}$, while $\mathcal{W}_{\mathbf{m}}^{\natural}$ behaves well since it is a subgroup of $W_{n, 2}$. So first we show (3), and then prove (2) by making use of (3).

We also consider the variety $\mathcal{X}=G \times V$, where $V$ is an $n$-dimensional vector space over $\mathbf{k}$ (of any characteristic), and $G=G L(V)$. $G$ acts diagonally on $\mathcal{X}$. Put $\mathcal{X}_{\text {uni }}=G_{\text {uni }} \times V$, where $G_{\text {uni }}$ is the set of unipotent elements in $G$. The variety $\mathcal{X}_{\text {uni }}$ is called the enhanced nilpotent cone by Achar-Henderson $[\mathrm{AH}]$. It is known by $[\mathrm{AH}],[\mathrm{T}]$ that $\mathcal{X}_{\text {uni }}$ is $G$-stable, and the set of $G$-orbits is in bijection with $\mathcal{P}_{n, 2}$. For each $\mathbf{m}=\left(m_{1}, m_{2}\right) \in \mathcal{Q}_{n, 2}$, one can define a similar map $\pi_{1}^{(\mathbf{m})}: \widetilde{\mathcal{X}}_{\mathbf{m} \text {,uni }} \rightarrow \mathcal{X}_{\mathbf{m} \text {,uni as }}$ in the exotic case. Achar-Henderson [AH] and Finkelberg-Ginzburg-Travkin [FGT] proved the Springer correspondence between the set of irreducible representaions of $S_{m_{1}} \times S_{m_{2}}$ and the set of simple perverse sheaves associated to the $G$-orbits in $\mathcal{X}_{\mathbf{m} \text {,uni }}$, which are direct summands of $\left(\pi_{1}^{(\mathbf{m})}\right)_{!} \overline{\mathrm{Q}}_{l}\left[\operatorname{dim} \mathcal{X}_{\mathbf{m}, \text { uni }}\right]$. In this paper, we consider the variety $\mathcal{X}=G \times V^{r-1}$ with the diagonal $G$-action, which we call the enhanced space of level $r$. The arguments used to prove the Springer correspondence (3) in the exotic case can be applied also to the enhanced case, step by step, by a suitable modification. Actually the argument becomes drastically simple. We show that the Springer correspondence holds for $\mathcal{W}_{\mathbf{m}}^{\natural}=S_{m_{1}} \times \cdots \times S_{m_{r}}$ for any $\mathbf{m} \in \mathcal{Q}_{n, r}$. (This result was announced by the author in 2009, but was not published.) In [Li], Li established the Springer correspondence for such $\mathcal{W}_{\mathbf{m}}^{\natural}$ in connection with certain perverse sheaves arising from the framed Jordan quiver. Considering the framed Jordan quiver is essentially the same as considering the enhanced variety. So in this case our result is regarded as an alternate approach for his result.

\section{Contents}

1. Intersection cohomology on $G_{\text {reg }}^{\iota \theta} \times V^{r-1}$ (exotic case)

2. Intersection cohomology on $G^{\iota \theta} \times V^{r-1}$ (exotic case)

3. A variant of Theorem 2.2

4. Intersection cohomology on $G^{\iota \theta} \times V^{r-1}$ (enhanced case)

5. Unipotent variety of enhanced type

6. Unipotent variety of exotic type

7. Springer correspondence

8. Determiniation of the Springer correspsondence 


\section{Intersection COHOMOlogy on $G_{\mathrm{reg}}^{\iota \theta} \times V^{r-1}$ (EXotic CASE)}

1.1. Let $\mathbf{k}$ be an algebraically closed field. In this paper, we consider the following two cases.

\section{(I) The exotic case.}

Let $V$ be a $2 n$ dimensional vector space over $\mathbf{k}$ (with char $\mathbf{k} \neq 2$ ), with basis $\left\{e_{1}, \ldots e_{n}, f_{1}, \ldots f_{n}\right\}$. Let $G=G L_{2 n}$. Consider an involutive automorphism $\theta: G \rightarrow$ $G$ given by

$$
\theta(g)=J^{-1}\left({ }^{t} g^{-1}\right) J \quad \text { with } J=\left(\begin{array}{cc}
0 & 1_{n} \\
-1_{n} & 0
\end{array}\right)
$$

where $1_{n}$ is the identity matrix of degree $n$, and put $H=G^{\theta}$. Then $H$ is the symplectic group $S p_{2 n}$ with respect to the symplectic form $\langle u, v\rangle={ }^{t} u J v$ for $u, v \in V$ under the identification $V \simeq \mathbf{k}^{2 n}$ via the basis $\left\{e_{1}, \ldots, e_{n}, f_{1}, \ldots f_{n}\right\}$, which gives rise to a symplectic basis.

Let $\iota: G \rightarrow G$ be the anti-automorphism $g \rightarrow g^{-1}$. We consider the set $G^{\iota \theta}=\left\{g \in G \mid \theta(g)=g^{-1}\right\}$. It is known that $G^{\iota \theta}=\left\{g \theta(g)^{-1} \mid g \in G\right\}$, and so $G^{\iota \theta} \simeq G / H$. Let $T \subset B$ be a pair of a $\theta$-stable maximal torus and a $\theta$-stable Borel subgroup of $G$. Let $M_{1} \subset \cdots \subset M_{n}$ be an isotropic flag in $V$ such that its stabilizer in $H$ coincides with $B^{\theta}$. We assume that $M_{i}=\left\langle e_{1}, \ldots, e_{i}\right\rangle$ for $i=1, \ldots n$, and that $e_{i}, f_{j}$ are weight vectors for $T$.

(II) The enhanced case.

Let $\widetilde{V}=V \oplus V$, where $V$ is an $n$-dimensional vector space over $\mathbf{k}$, and $G=$ $G_{0} \times G_{0}$ a subgroup of $G L(\widetilde{V})$ with $G_{0}=G L(V)$. Let $\theta: G \rightarrow G$ be an involution defined by $\theta\left(g_{1}, g_{2}\right)=\left(g_{2}, g_{1}\right)$. Put $H=G^{\theta} \simeq G_{0}$. Then $H$ acts naturally on $V$. Let $G^{\iota \theta}$ be a subset of $G$ defined similarly to the case (I). Then $G^{\iota \theta} \simeq G_{0}$ (as a set) and $H \simeq G_{0}$ acts on $G^{\iota \theta}$ by conjugation. Let $T \subset B$ be a pair of a $\theta$-stable maximal torus and a $\theta$-stable Borel subgroup of $G$. We can write $T=T_{0} \times T_{0}$ and $B=B_{0} \times B_{0}$ so that $B^{\theta} \simeq B_{0}, T^{\theta} \simeq T_{0}$. Let $M_{1} \subset \cdots \subset M_{n}=V$ be a complete flag in $V$ such that its stabilizer in $H$ coincides with $B^{\theta}$. We fix a basis $\left\{e_{1}, \ldots, e_{n}\right\}$ of $V$ such that $M_{i}=\left\langle e_{1}, \ldots, e_{i}\right\rangle$ for $i=1, \ldots n$, and that $e_{i}$ are weight vectors for $T^{\theta}$. Let $\left\{e_{1}, \ldots, e_{n}, f_{1}, \ldots, f_{n}\right\}$ be a basis of $\tilde{V}$, where $f_{i}=e_{i}$ for each $i$, and define a symplectic form $\langle$,$\rangle on \widetilde{V}$ so that $\left\{e_{i}, f_{j}\right\}$ gives a symplectic basis of $\widetilde{V}$.

1.2. For an integer $r \geq 1$, we consider the variety $G^{\iota \theta} \times V^{r-1}$ on which $H$ acts diagonally. We call $G^{\iota \theta} \times V^{r-1}$ the exotic symmetric space of level $r$ in the case (I), and the enhanced space of level $r$ in the case (II). Let $Q_{n, r}=\left\{\mathbf{m}=\left(m_{1}, \ldots, m_{r}\right) \in\right.$ $\left.\mathbf{Z}_{\geq 0}^{r} \mid \sum_{i} m_{i}=n\right\}$. We define $\mathcal{Q}_{n, r}^{0}=\left\{\mathbf{m} \in \mathcal{Q}_{n, r} \mid m_{r}=0\right\}$. For each $\mathbf{m} \in \mathcal{Q}_{n, r}$, we define $\mathbf{p}(\mathbf{m})=\left(p_{1}, p_{2}, \ldots, p_{r}\right)$ by $p_{i}=m_{1}+\cdots+m_{i}$ for each $i$. We define varieties

$$
\begin{aligned}
& \widetilde{\mathcal{X}}_{\mathbf{m}}=\left\{\left(x, \mathbf{v}, g B^{\theta}\right) \in G^{\iota \theta} \times V^{r-1} \times H / B^{\theta} \mid g^{-1} x g \in B^{\iota \theta}, g^{-1} \mathbf{v} \in \prod_{i=1}^{r-1} M_{p_{i}}\right\} \\
& \mathcal{X}_{\mathbf{m}}=\bigcup_{g \in H} g\left(B^{\iota \theta} \times \prod_{i=1}^{r-1} M_{p_{i}}\right) .
\end{aligned}
$$


We define a map $\pi^{(\mathbf{m})}: \widetilde{\mathcal{X}}_{\mathbf{m}} \rightarrow G^{\iota \theta} \times V^{r-1}$ by $\pi^{(\mathbf{m})}\left(x, \mathbf{v}, g B^{\theta}\right)=(x, \mathbf{v})$. Clearly $\mathcal{X}_{\mathbf{m}}=\operatorname{Im} \pi^{(\mathbf{m})}$. Since $\widetilde{\mathcal{X}}_{\mathbf{m}} \simeq H \times^{B^{\theta}}\left(B^{\iota \theta} \times \prod_{i} M_{p_{i}}\right), \widetilde{\mathcal{X}}_{\mathbf{m}}$ is smooth and irreducible. Since $\pi^{(\mathbf{m})}$ is proper, $\mathcal{X}_{\mathbf{m}}$ is a closed irreducible subvariety of $G^{\iota \theta} \times V^{r-1}$. In the case where $\mathbf{m}=(n, 0, \ldots, 0)$, namely, $\mathbf{p}(\mathbf{m})=(n, \ldots, n)$, we write $\widetilde{\mathcal{X}}_{\mathbf{m}}, \mathcal{X}_{\mathbf{m}}$ and $\pi^{(\mathbf{m})}$ by $\widetilde{\mathcal{X}}, \mathcal{X}$ and $\pi$, respectively. Note that for any $\mathbf{m} \in \mathcal{Q}_{n, r}, \mathcal{X}_{\mathbf{m}}$ is contained in $\mathcal{X}$. The dimension of $\widetilde{\mathcal{X}}_{\mathbf{m}}$ is computed as follows;

$$
\operatorname{dim} \widetilde{\mathcal{X}}_{\mathbf{m}}= \begin{cases}2 n^{2}+\sum_{i=1}^{r}(r-i) m_{i} & \text { exotic case } \\ n^{2}+\sum_{i=1}^{r}(r-i) m_{i} & \text { enhamced case }\end{cases}
$$

In fact, in the exotic case, by [SS1; (3.1.1)], we have

$$
\begin{aligned}
\operatorname{dim} \widetilde{\mathcal{X}}_{\mathbf{m}} & =\operatorname{dim} H / B^{\theta}+\operatorname{dim} B^{\iota \theta}+\sum_{i=1}^{r-1} \operatorname{dim} M_{p_{i}} \\
& =2 n^{2}+\sum_{i=1}^{r-1}\left(m_{1}+\cdots+m_{i}\right) \\
& =2 n^{2}+\sum_{i=1}^{r}(r-i) m_{i}
\end{aligned}
$$

The computation for the enhanced case is similar (in this case, $\operatorname{dim} B^{\theta}=\operatorname{dim} B^{\iota \theta}$ ).

Let $T_{\text {reg }}^{\iota \theta}$ be the set of regular semisimple elements in $T^{\llcorner\theta}$, namely, the set of elements in $T^{\llcorner\theta}$ such that all the eigenspaces in $V$ have dimension 2 (resp. dimension 1 ) in the exotic case (resp. in the enhanced case). We put $G_{\mathrm{reg}}^{\iota \theta}=\bigcup_{g \in H} g T_{\mathrm{reg}}^{\iota \theta} g^{-1}$, $B_{\text {reg }}^{\iota \theta}=G_{\text {reg }}^{\iota \theta} \cap B$. We define varieties $\widetilde{\mathcal{Y}}_{\mathbf{m}}, \mathcal{Y}_{\mathbf{m}}$ by

$$
\begin{aligned}
& \widetilde{\mathcal{Y}}_{\mathbf{m}}=\left\{\left(x, \mathbf{v}, g B^{\theta}\right) \in G_{\mathrm{reg}}^{\iota \theta} \times V^{r-1} \times H / B^{\theta} \mid g^{-1} x g \in B_{\mathrm{reg}}^{\iota \theta}, g^{-1} \mathbf{v} \in \prod_{i=1}^{r-1} M_{p_{i}}\right\} \\
& \mathcal{Y}_{\mathbf{m}}=\bigcup_{g \in H} g\left(B_{\mathrm{reg}}^{\iota \theta} \times \prod_{i=1}^{r-1} M_{p_{i}}\right),
\end{aligned}
$$

and a map $\psi^{(\mathbf{m})}: \widetilde{\mathcal{Y}}_{\mathbf{m}} \rightarrow G^{\iota \theta} \times V^{r-1}$ by $\psi^{(\mathbf{m})}\left(x, \mathbf{v}, g B^{\theta}\right)=(x, \mathbf{v})$. Clearly $\operatorname{Im} \psi^{(\mathbf{m})}=$ $\mathcal{Y}_{\mathbf{m}}$. As in the case of $\widetilde{\mathcal{X}}_{\mathbf{m}}$, we write $\widetilde{\mathcal{Y}}_{\mathbf{m}}, \mathcal{Y}_{\mathbf{m}}$ and $\psi^{(\mathbf{m})}$ by $\widetilde{\mathcal{Y}}, \mathcal{Y}, \psi$ in the case where $\mathbf{m}=(n, 0, \ldots, 0)$, . As in $[\mathrm{SS} 1 ;(3.1 .2)], \widetilde{\mathcal{Y}}_{\mathbf{m}}$ can be expressed as

$$
\begin{aligned}
\tilde{\mathcal{Y}}_{\mathbf{m}} & \simeq H \times{ }^{B^{\theta}}\left(B_{\mathrm{reg}}^{\iota \theta} \times \prod_{i} M_{p_{i}}\right) \\
& \simeq H \times \times^{B^{\theta} \cap Z_{H}\left(T^{\iota \theta}\right)}\left(T_{\mathrm{reg}}^{\iota \theta} \times \prod_{i} M_{p_{i}}\right) .
\end{aligned}
$$


1.3. In the remainder of this section, we assume that $\mathcal{X}$ is of exotic type. We define a partial order on $\mathcal{Q}_{n, r}$ by $\mathbf{m} \leq \mathbf{m}^{\prime}$ if $p_{i} \leq p_{i}^{\prime}$ for each $i$, where $\mathbf{p}(\mathbf{m})=$ $\left(p_{1}, \ldots, p_{r}\right)$ and $\mathbf{p}\left(\mathbf{m}^{\prime}\right)=\left(p_{1}^{\prime}, \ldots, p_{r}^{\prime}\right)$, respectively. Then we have $\mathcal{Y}_{\mathbf{m}} \subseteq \mathcal{Y}_{\mathbf{m}^{\prime}}$ if and only if $\mathbf{m} \leq \mathbf{m}^{\prime}$. Assume that $\mathbf{m} \leq \mathbf{m}^{\prime}$, then $\mathcal{X}_{\mathbf{m}}$ is a closed subset of $\mathcal{X}_{\mathbf{m}^{\prime}}$. Since $\mathcal{Y}_{\mathbf{m}}=\mathcal{Y} \cap \mathcal{X}_{\mathbf{m}}, \mathcal{Y}_{\mathbf{m}}$ is a closed subset of $\mathcal{Y}_{\mathbf{m}^{\prime}}$. We define a subset $\mathcal{Y}_{\mathbf{m}}^{0}$ of $\mathcal{Y}_{\mathbf{m}}$ by $\mathcal{Y}_{\mathbf{m}}^{0}=\mathcal{Y}_{\mathbf{m}} \backslash \bigcup_{\mathbf{m}^{\prime}<\mathbf{m}} \mathcal{Y}_{\mathbf{m}^{\prime}}$. Thus $\mathcal{Y}_{\mathbf{m}}^{0}$ is an open dense subset of $\mathcal{Y}_{\mathbf{m}}$, and we have a partition $\mathcal{Y}=\coprod_{\mathbf{m} \in \mathcal{Q}_{n, r}} \mathcal{Y}_{\mathbf{m}}^{0}$.

As in [SS1; 3.2], for each subset $I \subset[1, n]$, put $M_{I}=\left\{v \in M_{n} \mid \operatorname{supp}(v)=I\right\}$, where for $v=\sum_{i=1}^{n} a_{i} e_{i} \in M_{n}, \operatorname{supp}(v)$ is the set of $j \in[1, n]$ such that $a_{j} \neq 0$. $M_{I}$ is an open dense subset of the space spanned by $\left\{e_{i} \mid i \in I\right\}$, which we denote by $\bar{M}_{I}$. For each $\mathbf{m} \in \mathcal{Q}_{n, r}$, we define $\mathcal{I}(\mathbf{m})$ as the set of $\mathbf{I}=\left(I_{1}, \ldots, I_{r}\right)$ such that $[1, n]=\coprod_{i=1}^{r} I_{i}$ with $\left|I_{i}\right|=m_{i}$. For $\mathbf{I}=\left(I_{1}, \ldots, I_{r}\right)$, put $I_{<i}=I_{1} \cup I_{2} \cup \cdots \cup I_{i-1}$. Hence $\left|I_{<i}\right|=p_{i-1}$. For each $\mathbf{I} \in \mathcal{I}(\mathbf{m})$, we define a set $\mathbf{M}_{\mathbf{I}} \subset\left(M_{n}\right)^{r-1}$ by

$$
\mathbf{M}_{\mathbf{I}}=\left\{\mathbf{v}=\left(v_{1}, \ldots v_{r-1}\right) \in\left(M_{n}\right)^{r-1} \mid v_{i} \in M_{I_{i}}+\bar{M}_{I_{<i}}\right\}
$$

and define a variety $\widetilde{\mathcal{Y}}_{\mathbf{I}}$ by

$$
\widetilde{\mathcal{Y}}_{\mathbf{I}}=H \times{ }^{B^{\theta} \cap Z_{H}\left(T^{\iota \theta}\right)}\left(T_{\text {reg }}^{\iota \theta} \times \mathbf{M}_{\mathbf{I}}\right)
$$

Note that $Z_{H}\left(T^{\iota \theta}\right) \simeq S L_{2} \times \cdots \times S L_{2}$ (n-times) and $B^{\theta} \cap Z_{H}\left(T^{\iota \theta}\right)$ can be identified with the subgroup $B_{2} \times \cdots \times B_{2}$, where $B_{2}$ is the Borel subgroup of $S L_{2}$. Since the action of $B^{\theta} \cap Z_{H}\left(T^{\iota \theta}\right)$ on $M_{n}$ is given by the action of the torus part $T^{\theta}$, $\widetilde{\mathcal{Y}}_{\mathbf{I}}$ is well-defined. Let $\psi_{\mathbf{I}}: \widetilde{\mathcal{Y}}_{\mathbf{I}} \rightarrow \mathcal{Y}$ be the map induced from the map given by $(g,(t, \mathbf{v})) \mapsto\left(g t g^{-1}, g \mathbf{v}\right), H \times\left(T_{\text {reg }}^{\llcorner\theta} \times \mathbf{M}_{\mathbf{I}}\right) \rightarrow \mathcal{Y}$. Then $\operatorname{Im} \psi_{\mathbf{I}}$ coincides with $\mathcal{Y}_{\mathbf{m}}^{0}$ (independent of $\mathbf{I})$. For $\mathbf{I} \in \mathcal{I}(\mathbf{m})$, we define a parabolic subgroup $Z_{H}\left(T^{\iota \theta}\right)_{\mathbf{I}}$ of $Z_{H}\left(T^{\iota \theta}\right)$ by the condition that the $i$-th factor is $S L_{2}$ if $i \in I_{r}$ and is $B_{2}$ otherwise. Since $Z_{H}\left(T^{\iota \theta}\right)_{\mathbf{I}}$ stabilizes $\mathbf{M}_{\mathbf{I}}$, one can define

$$
\widehat{\mathcal{Y}}_{\mathbf{I}}=H \times{ }^{Z_{H}\left(T^{\iota \theta}\right)_{\mathbf{I}}}\left(T_{\text {reg }}^{\iota \theta} \times M_{\mathbf{I}}\right) .
$$

Then the map $\psi_{\mathbf{I}}$ factors through $\widehat{\mathcal{Y}}_{\mathbf{I}}$,

$$
\psi_{\mathbf{I}}: \widetilde{\mathcal{Y}}_{\mathbf{I}} \stackrel{\xi_{\mathbf{I}}}{\longrightarrow} \widehat{\mathcal{Y}}_{\mathbf{I}} \stackrel{\eta_{\mathbf{I}}}{\longrightarrow} \mathcal{Y}_{\mathbf{m}}^{0},
$$

where $\xi_{\mathbf{I}}$ is the natural projection and $\eta_{\mathbf{I}}$ is the map induced from the map $(g,(t, \mathbf{v})) \mapsto$ $\left(g t g^{-1}, g \mathbf{v}\right)$. Then $\xi_{\mathbf{I}}$ is a locally trivial fibration with fibre isomorphic to

$$
Z_{H}\left(T^{\iota \theta}\right)_{\mathbf{I}} /\left(B^{\theta} \cap Z_{H}\left(T^{\iota \theta}\right)\right) \simeq\left(S L_{2} / B_{2}\right)^{I_{r}} \simeq \mathbf{P}_{1}^{I_{r}},
$$

where $\left(S L_{2} / B_{2}\right)^{I_{r}}$ denotes the direct product of $S L_{2} / B_{2}$ with respect to the factors corrsponding to $I_{r}$, and similarly for $\mathbf{P}_{1}^{I_{r}}$. Thus $\mathbf{P}_{1}^{I_{r}}=\left(\mathbf{P}_{1}\right)^{m_{r}}$.

Let $S_{\mathbf{I}} \simeq S_{I_{1}} \times \cdots \times S_{I_{r}}$ be the stabilizer of $\left(I_{1}, \ldots, I_{r}\right)$ in $S_{n}$. Let $\mathcal{W}=$ $N_{H}\left(T^{\iota \theta}\right) / Z_{H}\left(T^{\iota \theta}\right) \simeq S_{n}$, and $\mathcal{W}_{\mathbf{I}}$ the subgroup of $\mathcal{W}$ corresponding to the subgroup $S_{\mathbf{I}}$. Then $\mathcal{W}$ acts on $Z_{H}\left(T^{\llcorner\theta}\right) \simeq S L_{2} \times \cdots \times S L_{2}$ as the permutation of factors, and 
$\mathcal{W}_{\mathbf{I}}$ stabilizes the group $Z_{H}\left(T^{\iota \theta}\right)_{\mathbf{I}}$. Since $\mathcal{W}_{\mathbf{I}}$ stabilizes $M_{\mathbf{I}}, \mathcal{W}_{\mathbf{I}}$ acts on $\widetilde{\mathcal{Y}}_{\mathbf{I}}$ and on $\widehat{\mathcal{Y}}_{\mathbf{I}}$. Now the map $\eta_{\mathbf{I}}: \widehat{\mathcal{Y}}_{\mathbf{I}} \rightarrow \mathcal{Y}_{\mathbf{m}}^{0}$ turns out to be a finite Galois covering with group $\mathcal{W}_{\mathbf{I}}$.

We define $\mathbf{I}(\mathbf{m})=\left(I_{1}^{\circ}, \ldots, I_{r}^{\circ}\right) \in \mathcal{I}(\mathbf{m})$ by $I_{i}^{\circ}=\left[p_{i-1}+1, p_{i}\right]$ for $i=1, \ldots, r$. For $\mathbf{I}=\mathbf{I}(\mathbf{m})$, put $\widetilde{\mathcal{Y}}_{\mathbf{I}}=\widetilde{\mathcal{Y}}_{\mathbf{m}}^{0}$ and $\mathcal{W}_{\mathbf{I}}=\mathcal{W}_{\mathbf{m}}$. Note that $\widetilde{\mathcal{Y}}_{\mathbf{m}}^{0}$ is an open dense subset of $\widetilde{\mathcal{Y}}_{\mathbf{m}}$, hnce irreducible. Put $\psi^{-1}\left(\mathcal{Y}_{\mathbf{m}}^{0}\right)=\widetilde{\mathcal{Y}}_{\mathbf{m}}^{+} \cdot \mathcal{W}$ acts naturally on $\widetilde{\mathcal{Y}}$ and the map $\psi$ is $\mathcal{W}$-equivariant with respect to the trivial action of $\mathcal{W}$ on $\mathcal{Y}$. Hence it preserves the subset $\widetilde{\mathcal{Y}}_{\mathbf{m}}^{+}$, and the stabilizer of $\widetilde{\mathcal{Y}}_{\mathbf{m}}^{0}$ in $\mathcal{W}$ coincides with $\mathcal{W}_{\mathbf{m}}$. One can check that

$$
\tilde{\mathcal{Y}}_{\mathbf{m}}^{+}=\coprod_{\mathbf{I} \in \mathcal{I}(\mathbf{m})} \widetilde{\mathcal{Y}}_{\mathbf{I}}=\coprod_{w \in \mathcal{W} / \mathcal{W}_{\mathbf{m}}} w\left(\widetilde{\mathcal{Y}}_{\mathbf{m}}^{0}\right)
$$

where $\widetilde{\mathcal{Y}}_{\mathbf{I}}$ is an irreducible component of $\widetilde{\mathcal{Y}}_{\mathbf{m}}^{+}$.

We have the following lemma (cf. [SS1, Lemma 3.3]).

Lemma 1.4. Assume that $r \geq 2$.

(i) $\mathcal{Y}_{\mathbf{m}}$ is open dense in $\mathcal{X}_{\mathbf{m}}$, and $\widetilde{\mathcal{Y}}_{\mathbf{m}}$ is open dense in $\widetilde{\mathcal{X}}_{\mathbf{m}}$.

(ii) $\operatorname{dim} \widetilde{\mathcal{X}}_{m}=\operatorname{dim} \widetilde{\mathcal{Y}}_{\mathbf{m}}=2 n^{2}+\sum_{i=1}^{r}(r-i) m_{i}$.

(iii) $\operatorname{dim} \mathcal{X}_{\mathbf{m}}=\operatorname{dim} \mathcal{Y}_{\mathbf{m}}=2 n^{2}+\sum_{i=1}^{r}(r-i) m_{i}-m_{r}$.

(iv) $\mathcal{Y}=\coprod_{\mathbf{m} \in \mathcal{Q}_{n, r}} \mathcal{Y}_{\mathbf{m}}^{0}$ gives a stratification of $\mathcal{Y}$ by smooth strata $\mathcal{Y}_{\mathbf{m}}^{0}$, and the map $\psi: \widetilde{\mathcal{Y}} \rightarrow \mathcal{Y}$ is semismall with respect to this stratification.

Proof. Since $B_{\text {reg }}^{\iota \theta} \times \prod_{i} M_{p_{i}}$ is open dense in $B^{\iota \theta} \times \prod_{i} M_{p_{i}}, \widetilde{\mathcal{Y}}_{\mathbf{m}}$ is open dense in $\widetilde{\mathcal{X}}_{\mathbf{m}}$. Since $\pi^{(\mathbf{m})}$ is a closed map and $\left(\pi^{(\mathbf{m})}\right)^{-1}\left(\mathcal{Y}_{\mathbf{m}}\right)=\widetilde{\mathcal{Y}}_{\mathbf{m}}, \mathcal{Y}_{\mathbf{m}}$ is open dense in $\mathcal{X}_{\mathbf{m}}$. So (i) holds. (ii) follows from (1.2.1). By using the decomposition $\psi_{\mathbf{I}}=\eta_{\mathbf{I}} \circ \xi_{\mathbf{I}}$ for $\mathbf{I}=\mathbf{I}(\mathbf{m})$, we see that $\operatorname{dim} \widetilde{\mathcal{Y}}_{\mathbf{m}}=\operatorname{dim} \mathcal{Y}_{\mathbf{m}}+m_{r}$. Hence (iii) holds. By (1.3.1) and (1.3.2), $\operatorname{dim} \psi^{-1}(x, \mathbf{v})=m_{r}$ for $(x, \mathbf{v}) \in \mathcal{Y}_{\mathbf{m}}^{0}$. Since

$$
\begin{aligned}
\operatorname{dim} \mathcal{Y}-\operatorname{dim} \mathcal{Y}_{\mathbf{m}}^{0} & =\left(2 n^{2}+(r-1) n\right)-\left(2 n^{2}+\sum_{i=1}^{r}(r-i) m_{i}-m_{r}\right) \\
& =\sum_{i=1}^{r}(r-1) m_{i}-\sum_{i=1}^{r}(r-i) m_{i}+m_{r} \\
& =\sum_{i=1}^{r}(i-1) m_{i}+m_{r} \\
& \geq 2 m_{r} .
\end{aligned}
$$

Hence $\operatorname{dim} \psi^{-1}(x, \mathbf{v}) \leq\left(\operatorname{dim} \mathcal{Y}-\operatorname{dim} \mathcal{Y}_{\mathbf{m}}^{0}\right) / 2$ for $(x, \mathbf{v}) \in \mathcal{Y}_{\mathbf{m}}^{0}$, and so $\psi$ is semismall.

1.5. Let $\psi_{\mathbf{m}}: \widetilde{\mathcal{Y}}_{\mathbf{m}}^{+} \rightarrow \mathcal{Y}_{\mathbf{m}}^{0}$ be the restriction of $\psi$ on $\widetilde{\mathcal{Y}}_{\mathbf{m}}^{+}$. Then $\psi_{\mathbf{m}}$ is $\mathcal{W}$ equivariant with respect to the natural action of $\mathcal{W}$ on $\widetilde{\mathcal{Y}}_{\mathbf{m}}^{+}$and the trivial action on $\mathcal{Y}_{\mathbf{m}}^{0}$. We consider the diagram

$$
T^{\iota \theta} \stackrel{\alpha_{0}}{\longleftarrow} \tilde{\mathcal{Y}} \stackrel{\psi}{\longrightarrow} \mathcal{Y}
$$


where $\alpha_{0}$ is given by $\alpha_{0}\left(x, \mathbf{v}, g B^{\theta}\right)=p_{T}\left(g^{-1} x g\right)\left(p_{T}: B^{\iota \theta} \rightarrow T^{\iota \theta}\right.$ is the natural projection). Let $\mathcal{E}$ be a tame local system on $T^{\iota \theta}$, and we consider the complex $\psi_{*} \alpha_{0}^{*} \mathcal{E}$ on $\mathcal{Y}$. For each $\mathbf{I} \in \mathcal{I}(\mathbf{m})$, we have a similar diagram as (1.5.1) by replacing $\widetilde{\mathcal{Y}}, \mathcal{Y}, \psi, \alpha_{0}$ by $\widetilde{\mathcal{Y}}_{\mathbf{I}}, \mathcal{Y}_{\mathbf{m}}^{0}, \psi_{\mathbf{I}}, \alpha_{\mathbf{I}}$. By (1.3.2), we have

$$
\left.\left.\left(\psi_{\mathbf{m}}\right) ! \alpha_{0}^{*} \mathcal{E}\right|_{\tilde{\mathcal{Y}}_{\mathbf{m}}^{+}} \simeq \bigoplus_{\mathbf{I} \in \mathcal{I}(\mathbf{m})}\left(\psi_{\mathbf{I}}\right) ! \alpha_{0}^{*} \mathcal{E}\right|_{\widetilde{\mathcal{Y}}_{\mathbf{I}}}
$$

Let $\mathcal{E}_{\mathbf{I}}=\left.\xi_{\mathbf{I}}^{*} \alpha_{0}^{*} \mathcal{E}\right|_{\widetilde{\mathcal{Y}}_{\mathbf{I}}}=\xi_{\mathbf{I}}^{*} \alpha_{\mathbf{I}}^{*} \mathcal{E}$ be a local system on $\widehat{\mathcal{Y}}_{\mathbf{I}}$, and $\mathcal{W}_{\mathcal{E}_{\mathbf{I}}}$ the stabilizer of $\mathcal{E}_{\mathbf{I}}$ in $\mathcal{W}_{\mathbf{I}}$. In the case where $\mathbf{I}=\mathbf{I}(\mathbf{m})$, we put $\mathcal{W}_{\mathcal{E}_{\mathbf{I}}}=\mathcal{W}_{\mathbf{m}, \mathcal{E}}$. In the case where $\mathbf{m}=(n, 0, \ldots, 0)$, we put $\mathcal{W}_{\mathbf{m}, \mathcal{E}}=\mathcal{W}_{\mathcal{E}}$, which is the stabilizer of $\mathcal{E}$ in $\mathcal{W}$. $\mathcal{W}_{\mathcal{E}}$ acts on $\left.\left(\psi_{\mathbf{m}}\right) ! \alpha_{0}^{*} \mathcal{E}\right|_{\tilde{\mathcal{Y}}_{\mathbf{m}}^{+}}$as automorphisms of complexes, and permutes each direct summand $\left(\psi_{\mathbf{I}}\right)_{!} \alpha_{\mathbf{I}}^{*} \mathcal{E}$ according to the permutaion action of $S_{n}$ on $\mathcal{I}(\mathbf{m})$. Since $\eta_{\mathbf{I}}$ is a finite Galois covering with group $\mathcal{W}_{\mathbf{I}},\left(\eta_{\mathbf{I}}\right) ! \mathcal{E}_{\mathbf{I}}$ is a semisimple local system. As in [SS1, 3.4] the endomorphism algebra $\operatorname{End}\left(\left(\eta_{\mathbf{I}}\right), \mathcal{E}_{\mathbf{I}}\right)$ is canonically isomorphic to the group algebra $\overline{\mathbf{Q}}_{l}\left[\mathcal{W}_{\mathcal{E}_{\mathbf{I}}}\right]$, and $\left(\eta_{\mathbf{I}}\right) ! \mathcal{E}_{\mathbf{I}}$ is decomposed as

$$
\left(\eta_{\mathbf{I}}\right)_{!} \mathcal{E}_{\mathbf{I}} \simeq \bigoplus_{\rho \in \mathcal{W}_{\mathcal{E}_{\mathbf{I}}}} \rho \otimes \mathcal{L}_{\rho}
$$

where $\mathcal{L}_{\rho}=\operatorname{Hom}\left(\rho,\left(\eta_{\mathbf{I}}\right)_{!} \mathcal{E}_{\mathbf{I}}\right)$ is a simple local system on $\mathcal{Y}_{\mathbf{m}}^{0}$.

1.6. Since $\psi_{\mathbf{m}}$ is proper and $\widetilde{\mathcal{Y}}_{\mathbf{I}}$ is closed in $\widetilde{\mathcal{Y}}_{\mathbf{m}}^{+}, \psi_{\mathbf{I}}$ is proper. Hence $\xi_{\mathbf{I}}$ is also proper. We note that

(1.6.1) $R^{i}\left(\xi_{\mathbf{I}}\right) ! \overline{\mathbf{Q}}_{l}$ is a constant sheaf for each $i$.

In fact, we have a commutative diagram

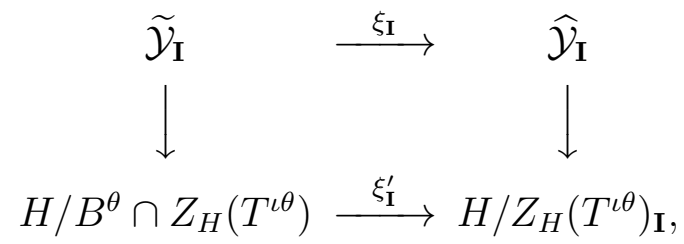

where vertical maps are natural projections (see 1.3), and the map $\xi_{\mathbf{I}}^{\prime}$ is the map induced from the inclusion $B^{\theta} \cap Z_{H}\left(T^{\iota \theta}\right) \hookrightarrow Z_{H}\left(T^{\iota \theta}\right)_{\mathbf{I}}$. Since this diagram is cartesian, (1.6.1) is equivalent to the statement that

(1.6.2) $R^{i}\left(\xi_{\mathbf{I}}^{\prime}\right)_{!} \overline{\mathbf{Q}}_{l}$ is a constant sheaf for each $i$.

We show (1.6.2). Since $\xi_{\mathbf{I}}^{\prime}$ is a locally trivial fibration, $R^{i}\left(\xi_{\mathbf{I}}^{\prime}\right)_{!} \overline{\mathbf{Q}}_{l}$ is a locally constant sheaf on $H / Z_{H}\left(T^{\iota \theta}\right)_{\mathbf{I}}$. Since $\xi_{\mathbf{I}}^{\prime}$ is $H$-equivariant, $R^{i}\left(\xi_{\mathbf{I}}^{\prime}\right) ! \overline{\mathbf{Q}}_{l}$ is an $H$-equivariant local system on $H / Z_{H}\left(T^{\iota \theta}\right)_{\mathbf{I}}$, hence it is a constant sheaf, as $Z_{H}\left(T^{\iota \theta}\right)_{\mathbf{I}}$ is connected. Thus (1.6.2), and so (1.6.1) holds.

Since $\xi_{\mathbf{I}}$ is a $\mathbf{P}_{1}^{I_{r}}$-bundle, we see that

$$
\left(\xi_{\mathbf{I}}\right) ! \alpha_{\mathbf{I}}^{*} \mathcal{E} \simeq H^{\bullet}\left(\mathbf{P}_{1}^{I_{r}}\right) \otimes \mathcal{E}_{\mathbf{I}}
$$


where $H^{\bullet}\left(\mathbf{P}_{1}^{I_{r}}\right)$ denotes $\bigoplus_{i>0} H^{2 i}\left(\mathbf{P}_{1}^{I_{r}}, \overline{\mathbf{Q}}_{l}\right)$, which we regard as a complex of vector spaces $\left(K_{i}\right)$ with $K_{\text {odd }}=0$. It follows that

$$
\left(\psi_{\mathbf{I}}\right)_{!} \alpha_{\mathbf{I}}^{*} \mathcal{E} \simeq\left(\eta_{\mathbf{I}}\right)_{!}\left(\xi_{\mathbf{I}}\right)_{!} \alpha_{\mathbf{I}}^{*} \mathcal{E} \simeq H^{\bullet}\left(\mathbf{P}_{1}^{I_{r}}\right) \otimes\left(\eta_{\mathbf{I}}\right)_{!} \mathcal{E}_{\mathbf{I}}
$$

Let $W_{n, r}=S_{n} \ltimes(\mathbf{Z} / r \mathbf{Z})^{n}$ be the complex reflection group $G(r, 1, n)$. We put $\widetilde{\mathcal{W}}=\mathcal{W} \ltimes(\mathbf{Z} / r \mathbf{Z})^{n}$. We define a subgroup $\widetilde{\mathcal{W}}_{\mathcal{E}}\left(\operatorname{resp} . \widetilde{\mathcal{W}}_{\mathbf{m}, \mathcal{E}}\right)$ of $\widetilde{\mathcal{W}}$ by $\widetilde{\mathcal{W}}_{\mathcal{E}}=$ $\mathcal{W}_{\mathcal{E}} \ltimes(\mathbf{Z} / r \mathbf{Z})^{n}$ (resp. $\left.\widetilde{\mathcal{W}}_{\mathbf{m}, \mathcal{E}}=\mathcal{W}_{\mathbf{m}, \mathcal{E}} \ltimes(\mathbf{Z} / r \mathbf{Z})^{n}\right)$. Let $\zeta$ be a primitive $r$-th root of unity in $\overline{\mathbf{Q}}_{l}$, and define a linear character $\tau_{i}: \mathbf{Z} / r \mathbf{Z} \rightarrow \overline{\mathbf{Q}}_{l}^{*}$ by $\tau_{i}(a)=\zeta^{i-1}$ for $i=1, \ldots, r$, where $a$ is a generator of $\mathbf{Z} / r \mathbf{Z}$. Let $\rho$ be an irreducible representation of $\mathcal{W}_{\mathbf{m}, \mathcal{E}}$. Since $\mathcal{W}_{\mathbf{m}, \mathcal{E}}$ is decomposed as $\mathcal{W}_{\mathbf{m}, \mathcal{E}}=\mathcal{W}_{1} \times \cdots \times \mathcal{W}_{r}$ with subgroups $\mathcal{W}_{i} \subset S_{m_{i}}, \rho$ can be written as $\rho=\rho_{1} \otimes \cdots \otimes \rho_{r}$ with $\rho_{i} \in \mathcal{W}_{i}^{\wedge}$. We define an irreducible $\widetilde{\mathcal{W}}_{\mathbf{m}, \mathcal{E}}$-module $\widetilde{\rho}$ (resp. $\widetilde{\rho}^{\prime}$ ) by defining the action of $(\mathbf{Z} / r \mathbf{Z})^{m_{i}}$ on $\rho_{i}$ via $\tau_{i}^{\otimes m_{i}}$ for $i=1, \ldots, r$ (resp. via $\tau_{i}^{\otimes m_{i}}$ for $i=1, \ldots, r-1$, and via the trivial action for $i=r)$. Put $\widetilde{V}_{\rho}=\operatorname{Ind} \widetilde{\mathcal{W}}_{\widetilde{\mathcal{W}}_{\mathbf{m}, \mathcal{E}}}^{\widetilde{\rho}_{\text {. }}}$. Then $\widetilde{V}_{\rho}$ is an irreducible $\widetilde{\mathcal{W}}_{\mathcal{E}}$-module.

We regard $H^{\bullet}\left(\mathbf{P}_{1}^{m_{r}}\right) \otimes \rho$ as a complex of $\mathcal{W}_{\mathbf{m}, \mathcal{E}}$-modules. We define an action of $\mathbf{Z} / r \mathbf{Z}$ on $H^{\bullet}\left(\mathbf{P}_{1}\right)=H^{2}\left(\mathbf{P}_{1}\right) \oplus H^{0}\left(\mathbf{P}_{1}\right) \simeq \overline{\mathbf{Q}}_{l} \oplus \overline{\mathbf{Q}}_{l}$ by $\tau_{r} \oplus \tau_{1}$, and define an action of $(\mathbf{Z} / r \mathbf{Z})^{m_{r}}$ on $H^{\bullet}\left(\mathbf{P}_{1}^{m_{r}}\right) \simeq H^{\bullet}\left(\mathbf{P}_{1}\right)^{\otimes m_{r}}$ by $\left(\tau_{r} \oplus \tau_{1}\right) \otimes \cdots \otimes\left(\tau_{r} \oplus \tau_{1}\right)\left(m_{r}\right.$-factors $)$. Thus we can consider an extension $H^{\bullet}\left(\mathbf{P}_{1}^{m_{r}}\right) \otimes \widetilde{\rho}$ of $H^{\bullet}\left(\mathbf{P}_{1}^{m_{r}}\right) \otimes \rho$, as a complex of $\widetilde{\mathcal{W}}_{\mathbf{m}, \mathcal{E}^{-}}$modules. In view of $(1.5 .2),(1.5 .3)$ and (1.6.4), we have

$$
\left.\left(\psi_{\mathbf{m}}\right)_{!} \alpha_{0}^{*} \mathcal{E}\right|_{\widetilde{\mathcal{Y}}_{\mathbf{m}}^{+}} \simeq \bigoplus_{\rho \in \mathcal{W}_{\mathbf{m}, \mathcal{E}}^{\wedge}} \operatorname{Ind}_{\widetilde{\mathcal{W}}_{\mathbf{m}, \mathcal{E}}}^{\widetilde{\mathcal{W}}_{\mathcal{E}}}\left(H^{\bullet}\left(\mathbf{P}_{1}^{m_{r}}\right) \otimes \widetilde{\rho}^{\prime}\right) \otimes \mathcal{L}_{\rho}
$$

Note that by our construction, (1.6.5) can be rewritten as

$$
\left.\left(\psi_{\mathbf{m}}\right) ! \alpha_{0}^{*} \mathcal{E}\right|_{\widetilde{\mathcal{Y}}_{\mathbf{m}}^{+}} \simeq\left(\bigoplus_{\rho \in \mathcal{W}_{\mathbf{m}, \mathcal{E}}^{\wedge}} \widetilde{V}_{\rho} \otimes \mathcal{L}_{\rho}\right)\left[-2 m_{r}\right]+\mathcal{N}_{\mathbf{m}}
$$

where $\mathcal{N}_{\mathbf{m}}$ is a sum of various $\mathcal{L}_{\rho}[-2 i]$ for $\rho \in \mathcal{W}_{\mathbf{m}, \mathcal{E}}^{\wedge}$ with $0 \leq i<m_{r}$.

For each $\mathbf{m} \in \mathcal{Q}_{n, r}$, let $\bar{\psi}_{\mathbf{m}}$ be the restriction of $\psi$ on $\psi^{-1}\left(\mathcal{Y}_{\mathbf{m}}\right)$. In what follows, we denote $\left.\alpha_{0}^{*} \mathcal{E}\right|_{\psi^{-1}\left(\mathcal{Y}_{\mathbf{m}}\right)}$ by $\alpha_{0}^{*} \mathcal{E}$ for short. Put $d_{\mathbf{m}}=\operatorname{dim} \mathcal{Y}_{\mathbf{m}}$. For $1 \leq j<r-1$, $0 \leq k \leq m_{j}$, we define a subset $\mathcal{Q}(\mathbf{m} ; j, k)$ of $\mathcal{Q}_{\mathbf{m}}$ by

$$
\mathcal{Q}(\mathbf{m} ; j, k)=\left\{\mathbf{m}^{\prime} \in \mathcal{Q}_{n, r} \mid \mathbf{m}^{\prime} \leq \mathbf{m}, p_{i}=p_{i}^{\prime}(1 \leq i \leq j-1), p_{j}^{\prime}=p_{j-1}+k\right\},
$$

where $\mathbf{p}(\mathbf{m})=\left(p_{1}, \ldots, p_{r}\right)$ and $\mathbf{p}\left(\mathbf{m}^{\prime}\right)=\left(p_{1}^{\prime}, \ldots, p_{r}^{\prime}\right)$. We also define $\mathbf{m}(j, k) \in \mathcal{Q}_{n, r}$ by $\mathbf{m}(j, k)=\left(m_{1}^{\prime}, \ldots, m_{r}^{\prime}\right)$ such that $m_{j}^{\prime}=k, m_{j+1}^{\prime}=m_{j+1}+\left(m_{j}-k\right)$ and $m_{i}^{\prime}=m_{i}$ for $i \neq j, j+1$. In the case where $j=r-1$, we write $\mathbf{m}(j, k)$ simply as $\mathbf{m}(k)$. Hence if $\mathbf{m} \in \mathcal{Q}_{n, r}^{0}, \mathbf{m}(k)=\left(m_{1}, \cdots, m_{r-2}, k, k^{\prime}\right)$ with $k+k^{\prime}=m_{r-1}$. We have the following proposition.

Proposition 1.7. For each $\mathbf{m} \in \mathcal{Q}_{n, r}^{0},\left(\bar{\psi}_{\mathbf{m}}\right)_{!} \alpha_{0}^{*} \mathcal{E}\left[d_{\mathbf{m}}\right]$ is a semisimple perverse sheaf on $\mathcal{Y}_{\mathbf{m}}$, equipped with $\widetilde{\mathcal{W}}_{\mathcal{E}}$-action, and is decomposed as 


$$
\left(\bar{\psi}_{\mathbf{m}}\right) ! \alpha_{0}^{*} \mathcal{E}\left[d_{\mathbf{m}}\right] \simeq \bigoplus_{0 \leq k \leq m_{r-1}} \bigoplus_{\rho \in \mathcal{W}_{\mathbf{m}(k), \mathcal{E}}^{\wedge}} \tilde{V}_{\rho} \otimes \operatorname{IC}\left(\mathcal{Y}_{\mathbf{m}(k)}, \mathcal{L}_{\rho}\right)\left[d_{\mathbf{m}(k)}\right]
$$

Proof. $\bar{\psi}_{\mathbf{m}}$ is proper, and a similar argument as in the proof of Lemma 1.4 (iv) shows that $\bar{\psi}_{\mathbf{m}}$ is semismall with respect to the stratification $\mathcal{Y}_{\mathbf{m}}=\coprod_{\mathbf{m}^{\prime} \leq \mathbf{m}} \mathcal{Y}_{\mathbf{m}^{\prime}}^{0}$ (note that $\left.m_{r}=0\right)$. It follows that $\left(\bar{\psi}_{\mathbf{m}}\right) ! \alpha_{0}^{*} \mathcal{E}\left[d_{\mathbf{m}}\right]$ is a semisimple perverse sheaf on $\mathcal{Y}_{\mathbf{m}}$.

For a given $\mathbf{m} \in \mathcal{Q}_{n, r}$ (not necessarily in $Q_{n, r}^{0}$ ) we define, for each integer $1 \leq$ $j \leq r-1$, and $0 \leq k \leq m_{j}$,

$$
\begin{aligned}
M^{(j, k)} & =\prod_{i=1}^{j-1}\left(M_{\left[p_{i-1}+1, p_{i}\right]}+M_{p_{i-1}}\right) \times\left(M_{\left[p_{j-1}+1, p_{j-1}+k\right]}+M_{p_{j-1}}\right) \times \prod_{i=j+1}^{r-1} M_{p_{i}}, \\
\mathcal{Y}_{j, k}^{0} & =\bigcup_{g \in H} g\left(T_{\mathrm{reg}}^{\iota \theta} \times M^{(j, k)}\right), \\
\tilde{\mathcal{Y}}_{j, k}^{+} & =\psi^{-1}\left(\mathcal{Y}_{j, k}^{0}\right),
\end{aligned}
$$

and let $\psi_{j, k}: \tilde{\mathcal{Y}}_{j, k}^{+} \rightarrow \mathcal{Y}_{j, k}^{0}$ be the restriciton of $\psi$ on $\tilde{\mathcal{Y}}_{j, k}^{+}$. (As a convention, we also consider the case where $j=0, k=0$. In which case $M^{(0,0)}=\prod_{i=1}^{r-1} M_{p_{i}}$.) Then $\psi_{j, k}$ is a proper map. $\mathcal{Y}_{j, k}^{0}$ coincides with $\mathcal{Y}_{\mathbf{m}}^{0}$ in the case where $j=r-1, k=m_{r-1}$, and coincides with $\mathcal{Y}_{\mathbf{m}}$ in the case where $j=0$ and $k=0$. We also conisder the varieties

$$
\begin{aligned}
\bar{M}^{(j, k)} & =\prod_{i=1}^{j-1}\left(M_{\left[p_{i-1}+1, p_{i}\right]}+M_{p_{i-1}}\right) \times M_{p_{j-1}+k} \times \prod_{i=j+1}^{r-1} M_{p_{i}} \\
\mathcal{Y}_{j, k} & =\bigcup_{g \in H} g\left(T_{\mathrm{reg}}^{\iota \theta} \times \bar{M}^{(j, k)}\right), \\
\overline{\widetilde{\mathcal{Y}}_{j, k}^{+}} & =\psi^{-1}\left(\mathcal{Y}_{j, k}\right),
\end{aligned}
$$

and let $\bar{\psi}_{j, k}: \overline{\widetilde{\mathcal{Y}}_{j, k}^{+}} \rightarrow \mathcal{Y}_{j, k}$ be the restriction of $\psi$ on $\overline{\widetilde{\mathcal{Y}}_{j, k}^{+}}$. Then $\mathcal{Y}_{j, k}^{0}$ is open dense in $\mathcal{Y}_{j, k}$. We have

(1.7.1) $\mathcal{Y}_{j, k} \backslash \mathcal{Y}_{j, k-1}=\mathcal{Y}_{j, k}^{0}$ if $k \geq 1$, and $\mathcal{Y}_{j, 0}(\mathbf{m})=\mathcal{Y}_{j+1, m_{j}+m_{j+1}}(\mathbf{m}(j, 0))$. Moreover, $\mathcal{Y}_{j, k}^{0}(\mathbf{m})$ coincides with $\mathcal{Y}_{j+1, m_{j+1}}(\mathbf{m}(j, k))$. (Here we use the notation $\mathcal{Y}_{j, k}(\mathbf{m})$, etc. to inidcate the dependence on $\mathbf{m}$.)

For $\mathbf{m}^{\prime} \in \mathcal{Q}(\mathbf{m} ; j, k), \mathcal{Y}_{\mathbf{m}^{\prime}}^{0}$ is contained in $\mathcal{Y}_{j, k}$. Hence one can define an intersection cohomology $\operatorname{IC}\left(\mathcal{Y}_{\mathbf{m}^{\prime}}^{\prime}, \mathcal{L}_{\rho}\right)$ associated to the local system $\mathcal{L}_{\rho}$ on $\mathcal{Y}_{\mathbf{m}^{\prime}}^{0}$ (here $\mathcal{Y}_{\mathbf{m}^{\prime}}^{\prime}$ denotes the closure of $\mathcal{Y}_{\mathbf{m}^{\prime}}^{0}$ in $\mathcal{Y}_{j, k}$ ). Returning to the setting in the proposition, we consider $\mathbf{m} \in \mathcal{Q}_{n, r}^{0}$. We show the following formulas. First assume that $j=r-1$ 
and $0 \leq k \leq m_{r-1}$. Then we have

$$
\begin{aligned}
& \left(\bar{\psi}_{r-1, k}\right) ! \alpha_{0}^{*} \mathcal{E} \\
& \quad \simeq \bigoplus_{0 \leq k^{\prime} \leq k} \bigoplus_{\rho \in \mathcal{W}_{\mathbf{m}\left(k^{\prime}\right), \mathcal{E}}^{\wedge}} \tilde{V}_{\rho} \otimes \operatorname{IC}\left(\mathcal{Y}_{r-1, k^{\prime}}, \mathcal{L}_{\rho}\right)\left[-2\left(m_{r-1}-k^{\prime}\right)\right]+\mathcal{N}_{r-1, k},
\end{aligned}
$$

where $\mathcal{N}_{r-1, k}$ is a sum of various $\operatorname{IC}\left(\mathcal{Y}_{r-1, k^{\prime}}, \mathcal{L}_{\rho}\right)[-2 i]$ for $0 \leq k^{\prime} \leq k$ and $\rho \in \mathcal{W}_{\mathbf{m}\left(k^{\prime}\right), \mathcal{E}}^{\wedge}$ with $0 \leq i<m_{r-1}-k^{\prime}$. Next assume that $0 \leq j<r-1$ and that $0 \leq k \leq m_{j}$. Then we have

$$
\stackrel{\left(\bar{\psi}_{j, k}\right)_{*} \alpha_{0}^{*} \mathcal{E}}{\simeq} \bigoplus_{0 \leq k^{\prime} \leq m_{r-1}} \bigoplus_{\rho \in \mathcal{W}_{\mathbf{m}\left(k^{\prime}\right), \mathcal{E}}^{\wedge}} \widetilde{V}_{\rho} \otimes \operatorname{IC}\left(\mathcal{Y}_{\mathbf{m}\left(k^{\prime}\right)}^{\prime}, \mathcal{L}_{\rho}\right)\left[-2\left(m_{r-1}-k^{\prime}\right)\right]+\mathcal{N}_{j, k}
$$

where $\mathcal{N}_{j, k}$ is a sum of various $\operatorname{IC}\left(\mathcal{Y}_{\mathbf{m}^{\prime}}^{\prime}, \mathcal{L}_{\rho}\right)[-2 i]$ for $\mathbf{m}^{\prime} \in \mathcal{Q}(\mathbf{m} ; j, k)$ and $\rho \in \mathcal{W}_{\mathbf{m}^{\prime}, \mathcal{E}}^{\wedge}$ with $i$ such that $0 \leq 2 i<d_{\mathbf{m}}-d_{\mathbf{m}^{\prime}}$.

Note that (1.7.3) will imply the proposition. In fact, in the case where $j=0, k=$ $0, \bar{\psi}_{j, k}$ coincides with $\bar{\psi}_{\mathbf{m}}$, and $\mathcal{Y}_{\mathbf{m}\left(k^{\prime}\right)}^{\prime}$ coincides with $\mathcal{Y}_{\mathbf{m}\left(k^{\prime}\right)}$. Take $\operatorname{IC}\left(\mathcal{Y}_{\mathbf{m}^{\prime}}, \mathcal{L}_{\rho}\right)[-2 i] \in$ $\mathcal{N}_{0,0}$. Since $d_{\mathbf{m}}-d_{\mathbf{m}^{\prime}}>2 i, \operatorname{IC}\left(\mathcal{Y}_{\mathbf{m}^{\prime}}, \mathcal{L}_{\rho}\right)\left[d_{\mathbf{m}}-2 i\right]$ is not a perverse sheaf. Since $\left(\bar{\psi}_{\mathbf{m}}\right)_{*} \alpha_{0}^{*} \mathcal{E}\left[d_{\mathbf{m}}\right]$ is a semisimple perverse sheaf, we conclude that $\mathcal{N}_{0,0}=0$. By Lemma 1.4 , (iii), we have $d_{\mathbf{m}}-d_{\mathbf{m}\left(k^{\prime}\right)}=2\left(m_{r-1}-k^{\prime}\right)$. Thus the proposition follows from (1.7.3).

First we show (1.7.2) by induction on $k$. Put $j=r-1$. In the case where $k=0$, $\mathcal{Y}_{j, 0}$ coincides with $\mathcal{Y}_{\mathbf{m}(j, 0)}^{0}$. Thus (1.7.2) follows from (1.6.5). We assume that (1.7.2) holds for any $k^{\prime}<k$. By $(1.7 .1), \mathcal{Y}_{j, k} \backslash \mathcal{Y}_{j, k-1} \simeq \mathcal{Y}_{j, k}^{0}=\mathcal{Y}_{\mathbf{m}(k)}^{0}$, and $\mathcal{Y}_{\mathbf{m}(k)}^{0}$ is an open dense subset of $\mathcal{Y}_{j, k}$. Since $\bar{\psi}_{j, k}$ is proper, $\left(\bar{\psi}_{j, k}\right)_{!} \alpha_{0}^{*} \mathcal{E}$ is a semisimple complex on $\mathcal{Y}_{j, k}$. Here we note that $\left(\bar{\psi}_{j, k}\right) ! \alpha_{0}^{*} \mathcal{E}$ has a natural structure of $\widetilde{\mathcal{W}}_{\mathcal{E}}$-complex. In fact, $\left(\psi_{\mathbf{m}(k)}\right) ! \alpha_{0}^{*} \mathcal{E}$ has a $\widetilde{\mathcal{W}}_{\mathcal{E}}$-action by (1.6.5). It induces a $\widetilde{\mathcal{W}}_{\mathcal{E}}$-action on $\left(\iota_{0} \circ \psi_{\mathbf{m}(k)}\right) ! \alpha_{0}^{*} \mathcal{E}$, where $\iota_{0}$ is an open immersion $\mathcal{Y}_{\mathbf{m}(k)}^{0} \hookrightarrow \mathcal{Y}_{j, k}$, and hence on its perverse chohomology ${ }^{p} H^{i}\left(\left(\iota_{0} \circ \psi_{\mathbf{m}(k)}\right) ! \alpha_{0}^{*} \mathcal{E}\right)$. On the other hand, by induction, $\left(\bar{\psi}_{j, k-1}\right) ! \alpha_{0}^{*} \mathcal{E}$ has a natural $\widetilde{\mathcal{W}}_{\mathcal{E}}$-action, which induces a $\widetilde{\mathcal{W}}_{\mathcal{E}}$-action on ${ }^{p} H^{i}\left(\left(\bar{\psi}_{j, k-1}\right) ! \alpha_{0}^{*} \mathcal{E}\right)$. Thus, by using the perverse cohomology exact sequence, one can define an action of $\widetilde{\mathcal{W}}_{\mathcal{E}}$ on ${ }^{p} H^{i}\left(\left(\bar{\psi}_{j, k}\right) ! \alpha_{0}^{*} \mathcal{E}\right)$. Since $\left(\bar{\psi}_{j, k}\right) ! \alpha_{0}^{*} \mathcal{E}$ is a semisimple complex, in this way the action of $\widetilde{\mathcal{W}}_{\mathcal{E}}$ on $\left(\bar{\psi}_{j, k}\right) ! \alpha_{0}^{*} \mathcal{E}$ can be defined.

Now, since $\left(\bar{\psi}_{j, k}\right) ! \alpha_{0}^{*} \mathcal{E}$ is a semisimple complex, it is a direct sum of the form $A[s]$ for a simple perverse sheaf $A$. Suppose that supp $A$ is not contained in $\mathcal{Y}_{j, k-1}$. Then supp $A \cap \mathcal{Y}_{\mathbf{m}(k)}^{0} \neq \emptyset$ and the restriction of $A$ on $\mathcal{Y}_{\mathbf{m}(k)}^{0}$ is a simple perverse sheaf on $\mathcal{Y}_{\mathbf{m}(k)}^{0}$. The restriction of $\left(\bar{\psi}_{j, k}\right)_{!} \alpha_{0}^{*} \mathcal{E}$ on $\mathcal{Y}_{\mathbf{m}(k)}^{0}$ is isomorphic to $\left(\psi_{\mathbf{m}(k)}\right)_{!} \alpha_{0}^{*} \mathcal{E}$. Hence its decomposition is given by the formula in (1.6.5). It follows that $\left.A\right|_{\mathcal{Y}_{\mathbf{m}(k)}^{0}}=\mathcal{L}_{\rho}\left[d_{j, k}\right]$ for some $\rho$ (here $\left.d_{j, k}=\operatorname{dim} \mathcal{Y}_{\mathbf{m}(k)}\right)$. This implies that $A=\operatorname{IC}\left(\mathcal{Y}_{j, k}, \mathcal{L}_{\rho}\right)\left[d_{j, k}\right]$ and that 
the direct sum of $A[s]$ appearing in $\left(\bar{\psi}_{j, k}\right) ! \alpha_{0}^{*} \mathcal{E}$ such that supp $A \cap \mathcal{Y}_{\mathbf{m}(k)}^{0} \neq \emptyset$ is given, in view of (1.6.6), by

$$
K_{1}=\bigoplus_{\rho \in \mathcal{W}_{\mathbf{m}(k), \mathcal{E}}^{\wedge}} \widetilde{V}_{\rho} \otimes \operatorname{IC}\left(\mathcal{Y}_{j, k}, \mathcal{L}_{\rho}\right)\left[-2\left(m_{r-1}-k\right)\right]+\mathcal{N}_{\mathbf{m}(k)}^{\prime}
$$

where $\mathcal{N}_{\mathbf{m}(k)}^{\prime}$ is a sum of various $\operatorname{IC}\left(\mathcal{Y}_{j, k}, \mathcal{L}_{\rho}\right)[-2 i]$ with $0 \leq i<m_{r-1}-k$.

If supp $A$ is contained in $\mathcal{Y}_{j, k-1}, A[s]$ appears as a direct summand of $\left(\bar{\psi}_{j, k-1}\right) ! \alpha_{0}^{*} \mathcal{E}$, which is decomposed as in (1.7.2) by induction hypothesis. Thus if we remove the contribution from the restriction of $K_{1}$, such $A[s]$ is determined from $\left(\bar{\psi}_{j, k-1}\right) ! \alpha_{0}^{*} \mathcal{E}$. So, we consider the restriction of $K_{1}$ on $\mathcal{Y}_{j, k-1}$. The summands $\operatorname{IC}\left(\mathcal{Y}_{j, k}, \mathcal{L}_{\rho}\right)[-2 i]$ in $\mathcal{N}_{\mathbf{m}(k)}^{\prime}$ are already contained in $\mathcal{N}_{r-1, k}$ if $0 \leq i<m_{r-1}-k$. So it is enough to consider $A=\operatorname{IC}\left(\mathcal{Y}_{j, k}, \mathcal{L}_{\rho}\right)\left[-2\left(m_{r-1}-k\right)\right]$. Note that the multiplicity space of $A$ in $K_{1}$ is $\widetilde{V}_{\rho}$. Hence the multiplicity space of a simple perverse sheaf $A^{\prime}$ appearing in the decomposition of $\left.A\right|_{\mathcal{Y}_{j, k-1}}$,up to shift, has a structure of $\widetilde{\mathcal{W}}_{\mathcal{E}}$-module which is a sum of $\widetilde{V}_{\rho}$. But by (1.7.2) applied for $k-1$, the multiplicity space of a simple perverse sheaf $B$ appearing in the first term of $\left(\bar{\psi}_{j, k-1}\right)_{!} \alpha_{0}^{*} \mathcal{E}$ is a sum of $\widetilde{V}_{\rho^{\prime}}$ with $\rho^{\prime} \in \mathcal{W}_{\mathbf{m}\left(k^{\prime}\right), \mathcal{E}}^{\wedge}$ for $k^{\prime}<k$. Thus $\left.A\right|_{\mathcal{Y}_{j, k-1}}$ gives no contribution on those first terms. This proves (1.7.2) for $k$. Hence (1.7.2) holds.

We now prove (1.7.3) by backwards induction on $j$ and induction on $k$. So assume that $j<r-1$. By $(1.7 .1), \mathcal{Y}_{j, 0}(\mathbf{m})=\mathcal{Y}_{j+1, m_{j}+m_{j+1}}(\mathbf{m}(j, 0))$. Hence by induction on $j$, we may assume that (1.7.3) holds for $\mathcal{Y}_{j, 0}$. Take $k \geq 1$, and assume that (1.7.3) holds for $k-1$. We have $\mathcal{Y}_{j, k} \backslash \mathcal{Y}_{j, k-1}=\mathcal{Y}_{j, k}^{0}$, and $\mathcal{Y}_{j, k}^{0}(\mathbf{m})=\mathcal{Y}_{j+1, m_{j+1}}(\mathbf{m}(j, k))$ by (1.7.1). Thus by induction on $j,\left(\psi_{j, k}\right) ! \alpha_{0}^{*} \mathcal{E}$ can be described by the formula in (1.7.3). In particular, $\left(\psi_{j, k}\right) ! \alpha_{0}^{*} \mathcal{E}$ is a semisimple complex consisting of $\operatorname{IC}\left(\mathcal{Y}_{\mathbf{m}^{\prime}}^{\prime}, \mathcal{L}_{\rho}\right)$, up to shift, for various $\mathbf{m}^{\prime} \in \mathcal{Q}(\mathbf{m} ; j, k)$. Similarly, by induction on $k,\left(\bar{\psi}_{j-1, k}\right) ! \alpha_{0}^{*} \mathcal{E}$ is described by (1.7.3), and it is a semisimple complex consisitng of $\operatorname{IC}\left(\mathcal{Y}_{\mathbf{m}^{\prime}}^{\prime}, \mathcal{L}_{\rho}\right)$, up to shift, for various $\mathbf{m}^{\prime} \in \mathcal{Q}(\mathbf{m} ; j, k-1)$. Let $K_{1}$ be a semisimple complex on $\mathcal{Y}_{j, k}$ obtained from $\left(\psi_{j, k}\right)_{!} \alpha_{0}^{*} \mathcal{E}$ as in (1.7.4). It is described by the formula (1.7.3) by replacing $\mathbf{m}$ by $\mathbf{m}^{\prime \prime}=\mathbf{m}(j, k)$. Here we note that

(1.7.5) Assume that $A$ is a direct summand of $K_{1}$. Then except the case where $A=\operatorname{IC}\left(\mathcal{Y}_{\mathbf{m}\left(k^{\prime}\right)}, \mathcal{L}_{\rho}\right)\left[-2\left(m_{r-1}-k^{\prime}\right)\right], A$ is contained in $\mathcal{N}_{j, k}$.

Assume that $A$ is a direct summand in the former part of $K_{1}$. Then $A=$ $\operatorname{IC}\left(\mathcal{Y}_{\mathbf{m}^{\prime \prime}\left(k^{\prime}\right)}, \mathcal{L}_{\rho}\right)\left[-2\left(m_{r-1}^{\prime \prime}-k^{\prime}\right)\right]$ for $0 \leq k^{\prime} \leq m_{r-1}^{\prime \prime}$, where we write $\mathbf{m}^{\prime \prime}=\left(m_{1}^{\prime \prime}, \ldots, m_{r}^{\prime \prime}\right)$. Then $\mathbf{p}\left(\mathbf{m}^{\prime \prime}\left(k^{\prime}\right)\right)=\left(p_{1}^{\prime}, \ldots, p_{r}^{\prime}\right)$, where $p_{i}=p_{i}^{\prime}$ for $i \neq j, r-1$, and $p_{j}^{\prime} \leq p_{j}, p_{r-1}^{\prime} \leq$ $p_{r-1}$. Then by Lemma 1.4 (iii), we have

$$
\begin{aligned}
d_{\mathbf{m}}-d_{\mathbf{m}^{\prime \prime}\left(k^{\prime}\right)} & =\left(p_{j}-p_{j}^{\prime}\right)+\left(p_{r-1}-p_{r-1}^{\prime}\right)+m_{r}^{\prime} \\
& \geq\left(p_{j}-p_{j}^{\prime}\right)+2 m_{r}^{\prime} .
\end{aligned}
$$

for $\mathbf{m}^{\prime \prime}\left(k^{\prime}\right)=\left(m_{1}^{\prime}, \ldots, m_{r}^{\prime}\right)$. (Note that $m_{r-1}^{\prime \prime}-k^{\prime}=m_{r}^{\prime}$.) Hence $d_{\mathbf{m}}-d_{\mathbf{m}^{\prime \prime}\left(k^{\prime}\right)}=$ $2\left(m_{r-1}^{\prime \prime}-k^{\prime}\right)$ if and only if $p_{j}=p_{j}^{\prime}$, i.e., $\mathbf{m}^{\prime \prime}\left(k^{\prime}\right)=\mathbf{m}\left(k^{\prime}\right)$. In that case $m_{r-1}^{\prime \prime}=m_{r-1}$. In all other cases, $A$ is contained in $\mathcal{M}_{j, k}$. Next assume that $A$ is a direct summand 
of the latter part of $K_{1}$. Thus $A$ is written as $A=\operatorname{IC}\left(\mathcal{Y}_{\mathbf{m}^{\prime}}^{\prime}, \mathcal{L}_{\rho}\right)[-2 i]$ for $\mathbf{m}^{\prime} \leq \mathbf{m}^{\prime \prime}$ with $d_{\mathbf{m}^{\prime \prime}}-d_{\mathbf{m}^{\prime}}>2 i$. But since $\mathbf{m}^{\prime \prime}=\mathbf{m}(j, k)$, we have $\mathbf{m}^{\prime \prime} \leq \mathbf{m}$ and $d_{\mathbf{m}} \geq d_{\mathbf{m}^{\prime \prime}}$. Hence $\mathbf{m}^{\prime} \leq \mathbf{m}$ and $d_{\mathbf{m}}-d_{\mathbf{m}^{\prime}} \geq d_{\mathbf{m}^{\prime \prime}}-d_{\mathbf{m}^{\prime}}>2 i$. This implies that $A$ is contained in $\mathcal{N}_{j, k} \cdot(1.7 .5)$ is proved.

Now (1.7.5) shows that the former part of $K_{1}$ coinicdes with the former part of $\left(\bar{\psi}_{j, k-1}\right) ! \alpha_{0}^{*} \mathcal{E}$. Hence by a similar argument as in the proof of (1.7.2), we obtain (1.7.3) for $(j, k)$. This proves (1.7.3), and so the proposition follows.

\section{Intersection COHOMOLOGy on $G^{\iota \theta} \times V^{r-1}$ (EXotic CASE)}

2.1. In this section we assume that $\mathcal{X}, \mathcal{Y}$ are of exotic type. We keep the notation in Section 1. For each $\mathbf{m} \in \mathcal{Q}_{n, r}^{0}$, we consider the complex $\left(\bar{\psi}_{\mathbf{m}}\right)_{!} \alpha_{0}^{*} \mathcal{E}\left[d_{\mathbf{m}}\right]$ as in Proposition 1.7. Under the notation there, $\mathcal{Y}_{\mathbf{m}(k)}^{0}$ is an open dense subset of $\mathcal{X}_{\mathbf{m}(k)}$. Hence one can consider the complex

$$
K_{\mathbf{m}, T, \mathcal{E}}=\bigoplus_{0 \leq k \leq m_{r-1}} \bigoplus_{\rho \in \mathcal{W}_{\mathbf{m}(k), \mathcal{E}}^{\wedge}} \tilde{V}_{\rho} \otimes \operatorname{IC}\left(\mathcal{X}_{\mathbf{m}(k)}, \mathcal{L}_{\rho}\right)\left[d_{\mathbf{m}(k)}\right]
$$

We consider the diagram

$$
T^{\iota \theta} \stackrel{\alpha}{\longleftarrow} \tilde{\mathcal{X}} \stackrel{\pi}{\longrightarrow} \mathcal{X}
$$

where $\alpha: \widetilde{\mathcal{X}} \rightarrow T^{\iota \theta}$ is defined by $\alpha\left(x, \mathbf{v}, g B^{\theta}\right)=p_{T}\left(g^{-1} x g\right)$. Let $\bar{\pi}_{\mathbf{m}}: \pi^{-1}\left(\mathcal{X}_{\mathbf{m}}\right) \rightarrow \mathcal{X}_{\mathbf{m}}$ be the rstriction of $\pi$ on $\pi^{-1}\left(\mathcal{X}_{\mathbf{m}}\right)$. We consider the complex $\left(\bar{\pi}_{\mathbf{m}}\right)_{!} \alpha^{*} \mathcal{E}\left[d_{\mathbf{m}}\right]$, where $\alpha^{*} \mathcal{E}$ is regarded as a local system on $\pi^{-1}\left(\mathcal{X}_{\mathbf{m}}\right)$ by restriction. THe following result is a generalization of [SS1, Theorem 4.2].

Theorem 2.2. For each $\mathbf{m} \in \mathcal{Q}_{n, r}^{0},\left(\bar{\pi}_{\mathbf{m}}\right)_{!} \alpha^{*} \mathcal{E}\left[d_{\mathbf{m}}\right] \simeq K_{\mathbf{m}, T, \mathcal{E}}$ as perverse sheaves on $\mathcal{X}_{\mathrm{m}}$.

2.3. The remainder of this section is devoted to the proof of the theorem. As in the case of $\mathcal{Y}_{\mathbf{m}}^{0}$, consider $\mathcal{X}_{\mathbf{m}}^{0}=\mathcal{X}_{\mathbf{m}} \backslash \bigcup_{\mathbf{m}^{\prime}<\mathbf{m}} \mathcal{X}_{\mathbf{m}^{\prime}}$ for each $\mathbf{m} \in \mathcal{Q}_{n, r}$. We shall describe the set $\mathcal{X}_{\mathbf{m}}^{0}$ explicitly. Put $\mathcal{X}_{\text {uni }}=\mathcal{X} \cap\left(G_{\text {uni }}^{\iota \theta} \times V^{r-1}\right)$ and we define $\mathcal{X}_{\mathbf{m} \text {,uni }}, \mathcal{X}_{\mathbf{m} \text {,uni }}^{0}$, etc. as the intersection of $\mathcal{X}_{\mathbf{m}}, \mathcal{X}_{\mathbf{m}}^{0}$, etc. with $\mathcal{X}_{\text {uni }}$. For $(x, v) \in$ $G_{\text {uni }}^{\iota \theta} \times V$, we denote by $\mathbf{k}[x] v$ the subspace of $V$ spanned by $v, x v, x^{2} v, \ldots$ First we note the following.

(2.3.1) For each $(x, \mathbf{v}) \in \mathcal{X}_{\text {uni }}$ with $\mathbf{v}=\left(v_{1}, \ldots, v_{r-1}\right)$, define a sequence $W_{1} \subset W_{2} \subset$ $\cdots \subset W_{r-1}$ of subspaces of $V$ by $W_{i}=\mathbf{k}[x] v_{1}+\cdots+\mathbf{k}[x] v_{i}$ for $i=1, \ldots, r-1$. Then $(x, \mathbf{v}) \in \mathcal{X}_{\mathbf{m} \text {,uni }}^{0}$ if and only if $\operatorname{dim} W_{i}=p_{i}$ for each $i$.

In fact, if $(x, \mathbf{v}) \in \mathcal{X}_{\mathbf{m} \text {,uni }}$, there exists an $x$-stable isotropic flag $\left(V_{i}\right)_{1 \leq i \leq n}$ such that $v_{i} \in V_{p_{i}}$. Hence we have $\operatorname{dim} W_{i} \leq p_{i}$ for each $i$. This implies that $(x, \mathbf{v})$ satisfying the condition on $\left(W_{i}\right)$ is contained in $\mathcal{X}_{\mathbf{m} \text {,uni }}^{0}$. Conversely, assume that $(x, \mathbf{v}) \in \mathcal{X}_{\mathbf{m} \text {,uni }}^{0}$. Take an $x$-stable isotropic flag $\left(V_{i}\right)$ such that $v_{i} \in V_{p_{i}}$. Suppose there exists $k$ such that $\operatorname{dim} W_{i}=p_{i}$ for $i<k$ and that $\operatorname{dim} W_{k}<p_{k}$. Then $W_{i}=V_{p_{i}}$ for $i=1, \ldots, k-1$, and $\mathbf{k}[x] v_{k}+V_{k-1}$ is an $x$-stable proper subspace of $V_{p_{k}}$. One 
can find an $x$-stable flag $V_{p_{k-1}} \subset V_{p_{k-1}+1}^{\prime} \subset \cdots \subset V_{p_{k}-1}^{\prime} \subset V_{p_{k}}$, and $v_{k} \in V_{j}^{\prime} \subsetneq V_{p_{k}}$ for some $j$. This implies that $(x, \mathbf{v}) \in \mathcal{X}_{\mathbf{m}^{\prime}}$ for $\mathbf{m}^{\prime}<\mathbf{m}$, a contradiction. Hence (2.3.1) holds.

More generally, we consider $(x, \mathbf{v}) \in \mathcal{X}_{\mathbf{m}}$. Let $x=s u=u s$ be the Jordan decomposition of $x \in G^{\iota \theta}$, where $s \in G^{\iota \theta}$ is semisimple, $u \in G^{\iota \theta}$ is unipotent. We consider the decoposition $V=V_{1} \oplus \cdots \oplus V_{t}$ into eigenspaces of $s$. Then $Z_{G}(s) \simeq$ $G L_{2 n_{1}} \times \cdots \times G L_{2 n_{t}}$ with $\operatorname{dim} V_{j}=2 n_{j}$. Put $G_{j}=G L_{2 n_{j}}$ for each $j$. Then $Z_{G}(s)$ is $\theta$-stable, and $\theta$ stabilizes each factor so that $Z_{H}(s) \simeq G_{1}^{\theta} \times \cdots \times G_{t}^{\theta}$ with $G_{j}^{\theta} \simeq S p_{2 n_{j}}$. Take $\mathbf{v}=\left(v_{1}, \ldots, v_{r-1}\right) \in V^{r-1}$. For $j=1, \ldots, t$, we define $\mathbf{v}_{j}=\left(v_{1, j}, \ldots v_{r-1, j}\right) \in$ $V^{r-1}$, where $v_{i j}$ is the projection of $v_{i} \in V$ on $V_{j}$. Let $u_{j}$ be the restriction of $u$ on $V_{j}$. Then $\left(u_{j}, \mathbf{v}_{j}\right) \in\left(G_{j}\right)_{\text {uni }}^{\iota \theta} \times V_{j}^{r-1}$. We denote by $\left(\mathcal{X}^{G_{j}}\right)_{\mathbf{m}_{j} \text {,uni }}^{0}$ the subvariety of $\left(G_{j}\right)_{\mathrm{uni}}^{\iota \theta} \times V_{j}^{r-1}$ defined in a similar way as $\mathcal{X}_{\mathbf{m} \text {,uni }}^{0}$. The following property is checked easily.

(2.3.2) Assume that $(x, \mathbf{v})$ is contained in $\mathcal{X}_{\mathbf{m}}^{0}$. Then there exist unique $\mathbf{m}_{1}, \ldots, \mathbf{m}_{t}$ such that $\left(u_{j}, \mathbf{v}_{j}\right) \in\left(\mathcal{X}^{G_{j}}\right)_{\mathbf{m}_{j} \text {,uni }}^{0}$, where $\mathbf{m}_{j}=\left(m_{1, j}, \ldots m_{r, j}\right)$ with $\sum_{j=1}^{t} m_{i j}=m_{i}$ for $1 \leq i \leq r$. Coversely, if $\left(u_{j}, \mathbf{v}_{j}\right) \in\left(\mathcal{X}^{G_{j}}\right)_{\mathbf{m}_{j}, \text { uni }}^{0}$ for each $j$, then $(x, \mathbf{v}) \in \mathcal{X}_{\mathbf{m}}^{0}$ for $\mathbf{m}$ determined from $\mathbf{m}_{1}, \ldots, \mathbf{m}_{t}$.

For each $(x, \mathbf{v}) \in \mathcal{X}$, let $\left(u_{j}, \mathbf{v}_{j}\right)$ be defined as above. For $j=1, \ldots, t$, we define a flag $W_{1, j} \subset \cdots \subset W_{r-1, j}$ of $V_{j}$ with respect to $\left(u_{j}, \mathbf{v}_{j}\right)$ as in (2.3.1). Put $W_{i}=W_{i, 1} \oplus \cdots \oplus W_{i, t}$ for $i=1, \ldots, r-1$, then we obtain a sequence $W_{1} \subset \cdots \subset W_{r-1}$ of subspaces in $V$. We put $W_{i}(x, \mathbf{v})=W_{i}$ for each $i$. Then (2.3.2) can be rewritten as

$$
\mathcal{X}_{\mathbf{m}}^{0}=\left\{(x, \mathbf{v}) \in \mathcal{X} \mid \operatorname{dim} W_{i}(x, \mathbf{v})=p_{i}(1 \leq i \leq r-1)\right\}
$$

Recall the map $\pi: \widetilde{\mathcal{X}} \rightarrow \mathcal{X}$. For each $\mathbf{m} \in \mathcal{Q}_{n, r}$, we define $\widetilde{\mathcal{X}}_{\mathbf{m}}^{+}=\pi^{-1}\left(\mathcal{X}_{\mathbf{m}}^{0}\right)$, and let $\pi_{\mathbf{m}}: \widetilde{\mathcal{X}}_{\mathbf{m}}^{+} \rightarrow \mathcal{X}_{\mathbf{m}}^{0}$ be the restriction of $\pi$ on $\widetilde{\mathcal{X}}_{\mathbf{m}}^{+}$. Since $\mathcal{Y}_{\mathbf{m}}^{0}$ is open in $\mathcal{X}_{\mathbf{m}}^{0}, \widetilde{\mathcal{Y}}_{\mathbf{m}}^{+}$is an open subset of $\widetilde{\mathcal{X}}_{\mathbf{m}}^{+}$. For $\left(x, \mathbf{v}, g B^{\theta}\right) \in \widetilde{\mathcal{X}}_{\mathbf{m}}^{+}$, we shall associate $\mathbf{I} \in \mathcal{I}(\mathbf{m})$ as follows; assume that $(x, \mathbf{v}) \in B^{\iota \theta} \times M_{n}^{r-1}$, and that $x=s u$ is the Jordan decomposition of $x$. Then $M_{n}$ is $s$-stable, and is decomposed as $M_{n}=M_{n, 1} \oplus \cdots \oplus M_{n, t}$, where $M_{n, j}=M_{n} \cap V_{j}$ is a maximal isotropic subspace of $V_{j}$. Here $M_{n}=\left\langle e_{1}, \ldots e_{n}\right\rangle$. Since $s \in B^{\iota \theta}, M_{n, j}$ determines a set $\left\{e_{k_{1}}, \ldots, e_{k_{n_{j}}}\right\}$ with $k_{1}<k_{2}<\cdots<k_{n_{j}}$, where $n_{j}=\operatorname{dim} M_{n, j}$ (if $s_{1} \in T^{\iota \theta}$ is the projection of $s,\left\{e_{k_{1}}, \ldots, e_{k_{n_{j}}}\right\}$ are eigenvectors of $s_{1}$ on $\left.M_{n, j}\right)$. . Let $\left(u_{j}, \mathbf{v}_{j}\right)$ be as before. We define $\mathbf{I}=\left(I_{1}, \ldots, I_{r}\right) \in \mathcal{I}(\mathbf{m})$ as follows; let $\left(W_{i}\left(u_{j}, \mathbf{v}_{j}\right)\right)$ be as in (2.3.1) and put $p_{i j}=\operatorname{dim} W_{i}\left(u_{j}, \mathbf{v}_{j}\right)$. Then $W_{i}\left(u_{j}, \mathbf{v}_{j}\right)$ is a subspace of $M_{n, j}$. We define a subset $\widetilde{I}_{i, j}$ of $\left\{k_{1}, \ldots, k_{n_{j}}\right\}$ as the first $p_{i, j}$ numbers in $\left\{k_{1}, \ldots, k_{n_{j}}\right\}$, and define $I_{i, j}$ by $I_{i, j}=\widetilde{I}_{i, j} \backslash \widetilde{I}_{i-1, j}$. Thus $\left|I_{i, j}\right|=m_{i, j}$ for $\mathbf{m}_{j}=\left(m_{1, j}, \ldots m_{r, j}\right)$. Put $I_{i}=I_{i, 1} \amalg \cdots \coprod I_{i, t}$, and $\mathbf{I}=\left(I_{1}, \ldots, I_{r}\right)$. Then $\mathbf{I} \in \mathcal{I}(\mathbf{m})$. Note that the attachment $(x, \mathbf{v}) \mapsto \mathbf{I}$ depends only on the $B^{\theta}$-conjugacy class of $(x, \mathbf{v})$. Thus we have a well-defined map $\left(x, \mathbf{v}, g B^{\theta}\right) \mapsto \mathbf{I}$. We define a subvariety $\widetilde{\mathcal{X}}_{\mathbf{I}}$ of $\widetilde{\mathcal{X}}_{\mathrm{m}}^{+}$by

$$
\widetilde{\mathcal{X}}_{\mathbf{I}}=\left\{\left(x, \mathbf{v}, g B^{\theta}\right) \in \widetilde{\mathcal{X}}_{\mathbf{m}}^{+} \mid\left(x, \mathbf{v}, g B^{\theta}\right) \mapsto \mathbf{I}\right\}
$$


We show the following lemma (cf. [SS1, Lemma 4.4]).

Lemma 2.4. $\widetilde{\mathcal{X}}_{\mathbf{m}}^{+}$is decomposed as

$$
\widetilde{\mathcal{X}}_{\mathbf{m}}^{+}=\coprod_{\mathbf{I} \in \mathcal{I}(\mathbf{m})} \widetilde{\mathcal{X}}_{\mathbf{I}}
$$

where $\widetilde{\mathcal{X}}_{\mathbf{I}}$ is an irreducible component of $\widetilde{\mathcal{X}}_{\mathbf{m}}^{+}$for each $\mathbf{I}$.

Proof. It is clear from the definition that $\widetilde{\mathcal{X}}_{\mathbf{m}}^{+}$is a disjoint union of various $\widetilde{\mathcal{X}}_{\mathbf{I}}$, and that $\widetilde{\mathcal{X}}_{\mathbf{I}}$ contains $\widetilde{\mathcal{Y}}_{\mathbf{I}}$ as an open dense subset. Since $\widetilde{\mathcal{Y}}_{\mathbf{m}}^{+}=\coprod_{\mathbf{I}} \widetilde{\mathcal{Y}}_{\mathbf{I}}, \widetilde{\mathcal{Y}}_{\mathbf{m}}^{+}$is open dense in $\widetilde{\mathcal{X}}_{\mathbf{m}}^{+}$. Hence $\widetilde{\mathcal{X}}_{\mathbf{m}}=\bigcup_{\mathbf{I}} \overline{\mathcal{Y}}_{\mathbf{I}}$ gives a decomposition into irreducible components, where $\widetilde{\mathcal{Y}}_{\mathbf{I}}$ is the closure of $\widetilde{\mathcal{Y}}_{\mathbf{I}}$ in $\widetilde{\mathcal{X}}_{\mathbf{m}}^{+}$. Thus in order to show the lemma, it is enough to see that $\widetilde{\mathcal{X}}_{\mathbf{I}}$ is closed in $\widetilde{\mathcal{X}}_{\mathbf{m}}^{+}$for each $\mathbf{I}$. But the closure $\mathcal{Z}_{\mathbf{I}}$ of $\widetilde{\mathcal{X}}_{\mathbf{I}}$ in $\mathcal{X}$ is contained in the set $\widetilde{\mathcal{X}}_{\mathbf{I}} \cup \bigcup_{\mathbf{m}^{\prime}<\mathbf{m}} \widetilde{\mathcal{X}}_{\mathbf{m}^{\prime}}^{+}$. Hence $\widetilde{\mathcal{X}}_{\mathbf{I}}=\mathcal{Z}_{\mathbf{I}} \cap \widetilde{\mathcal{X}}_{\mathbf{m}}^{+}$is closed in $\widetilde{\mathcal{X}}_{\mathbf{m}}^{+}$.

2.5. We fix $\mathbf{m} \in \mathcal{Q}_{n, r}$. Let us consider the spaces $V_{0}=M_{m_{1}}$ and $\bar{V}_{0}=V_{0}^{\perp} / V_{0}$. We put $G_{1}=G L\left(V_{0}\right)$ and $G_{2}=G L\left(\bar{V}_{0}\right)$. Then $\bar{V}_{0}$ has a natural symplectic structure, and $G_{2}$ is identified with a $\theta$-stable subgroup of $G$. We consider the variety $\mathcal{X}^{\prime} \subset G_{2}^{\iota \theta} \times \bar{V}_{0}^{r-2}$ as in the case of $G^{\iota \theta} \times V^{r-1}$. Put $\mathbf{m}^{\prime}=\left(m_{2}, \ldots, m_{r}\right)$. Thus $\mathbf{m}^{\prime} \in \mathcal{Q}_{n^{\prime}, r-1}$, where $n^{\prime}=\operatorname{dim} \bar{V}_{0} / 2$. The subvariety $\mathcal{X}_{\mathbf{m}^{\prime}}^{\prime}$ of $\mathcal{X}^{\prime}$ with respect to $\mathbf{m}^{\prime}$ is defined similar to $\mathcal{X}_{\mathbf{m}}$. Let $G_{1}^{0}$ be the set of regular elements in $G_{1}$ (namely, the set of $x \in G_{1}$ such that $u$ is regular unipotent in $Z_{G_{1}}(s)$ for the Jordan decomposition $x=s u)$. For each $z=(x, \mathbf{v}) \in \mathcal{X}_{\mathbf{m}}^{0}$, put $W_{z}=W_{1}(x, \mathbf{v})$. Note that $W_{z}$ is an $x$-stable subspace of $V$ with $\operatorname{dim} W_{z}=m_{1}$, and that $\left.x\right|_{W_{z}}$ is a regular element in $G L\left(W_{z}\right)$. Moreover, $W_{z}$ is the unique $x$-stable subspace of $V$ containing $v_{1}$ with dimension $m_{1}$.

We define a variety

$$
\begin{aligned}
\mathcal{K}_{\mathbf{m}}= & \left\{\left(z, \phi_{1}, \phi_{2}\right) \mid z=(x, \mathbf{v}) \in \mathcal{X}_{\mathbf{m}}^{0}\right. \\
& \left.\phi_{1}: W_{z} \stackrel{\sim}{\rightarrow} V_{0}, \phi_{2}: W_{z}^{\perp} / W_{z} \stackrel{\sim}{\rightarrow} \bar{V}_{0} \text { (symplectic isom.) }\right\},
\end{aligned}
$$

and morphisms

$$
\begin{aligned}
& q: \mathcal{K}_{\mathbf{m}} \rightarrow \mathcal{X}_{\mathbf{m}}^{0}, \quad\left(x, \mathbf{v}, \phi_{1}, \phi_{2}\right) \mapsto(x, \mathbf{v}) \\
& \sigma: \mathcal{K}_{\mathbf{m}} \rightarrow G_{1}^{0} \times \mathcal{X}_{\mathbf{m}^{\prime}}^{\prime 0} \\
& \quad\left(x, \mathbf{v}, \phi_{1}, \phi_{2}\right) \mapsto\left(\phi_{1}\left(\left.x\right|_{W_{z}}\right) \phi_{1}^{-1}, \phi_{2}\left(\left.x\right|_{W_{z}^{\perp} / W_{z}}\right) \phi_{2}^{-1}, \phi_{2}(\overline{\mathbf{v}})\right),
\end{aligned}
$$

where $\overline{\mathbf{v}}=\left(\bar{v}_{2}, \ldots, \bar{v}_{r-1}\right) \in\left(W_{z}^{\perp} / W_{z}\right)^{r-2}$, and $\bar{v}_{i}$ is the image of $v_{i} \in W_{z}^{\perp}$ to $W_{z}^{\perp} / W_{z}$. Put $H_{0}=G_{1} \times G_{2}^{\theta}$. Then $H \times H_{0}$ acts on $\mathcal{K}_{\mathbf{m}}$ by

$$
\left(g,\left(h_{1}, h_{2}\right)\right):\left(x, \mathbf{v}, \phi_{1}, \phi_{2}\right) \mapsto\left(g x g^{-1}, g \mathbf{v}, h_{1} \phi_{1} g^{-1}, h_{2} \phi_{2} g^{-1}\right)
$$

for $g \in H,\left(h_{1}, h_{2}\right) \in H_{0}$. Moreover, $\sigma$ is $H \times H_{0}$-equivariant with respec to the natural action of $H_{0}$ and the trivial action of $H$ on $G_{1}^{0} \times \mathcal{X}_{\mathbf{m}^{\prime}}^{\prime}$. We have 
(2.5.2) The map $q$ is a principal bundle with fibre isomorphic to $H_{0}$.

(2.5.3) The map $\sigma$ is a locally trivial fibration with smooth fibre of dimension $\operatorname{dim} H+(r-2) m_{1}$.

In fact, (2.5.2) is clear. We show (2.5.3). For a fixd $z=\left(x^{\prime},\left(x^{\prime \prime}, \mathbf{v}^{\prime}\right)\right) \in G_{1}^{0} \times \mathcal{X}_{\mathbf{m}^{\prime}}^{\prime 0}$ with $\mathbf{v}^{\prime}=\left(v_{2}^{\prime}, \ldots, v_{r-1}^{\prime}\right)$, the fibre $\sigma^{-1}(z)$ is determined by the following procedure.

(i) Choose an isotropic subspace $W_{1}$ of $V$ with $\operatorname{dim} W_{1}=m_{1}$.

(ii) For such $W_{1}$, choose an isomorphism $\phi_{1}: W_{1} \rightarrow V_{0}$ and a symplectic isomorphism $\phi_{2}: W_{1}^{\perp} / W_{1} \rightarrow \bar{V}_{0}$.

(iii) Choose $x \in G^{\iota \theta}$ such that $\phi_{1}\left(\left.x\right|_{W_{1}}\right) \phi_{1}^{-1}=x^{\prime}, \phi_{2}\left(\left.x\right|_{W_{1}^{\perp} / W_{1}}\right) \phi_{2}^{-1}=x^{\prime \prime}$.

(iv) Choose $v_{1} \in W_{1}$ and $v_{i} \in W_{1}^{\perp}$ such that $\phi_{2}\left(\bar{v}_{i}\right)=v_{i}^{\prime}$ for $i=2, \ldots, r-1$.

Let $P$ be the stabilizer of the flag $\left(V_{0} \subset V_{0}^{\perp}\right)$ in $G$. Then $P$ is $\theta$-stable, and is decomposed as $P=L U_{P}$, where $L$ is a $\theta$-stable Levi subgorup of $P$ containing $T$ and $U_{P}$ is the unipotent radical of $P$. For (i), such $W_{1}$ are parametrized by $H / P^{\theta}$. For (ii), they are paramerrized by $G_{1} \times G_{2}^{\iota \theta}$. For (iii), $x$ should be contained in $P^{\iota \theta}$, but $x^{\prime}, x^{\prime \prime}$ determines the part corresponding to $L^{\iota \theta}$. Hence the choice of $x$ is parametrized by $U_{P}^{\iota \theta}$. Finally, $v_{1}$ is any element in $W_{1}, v_{2}, \ldots, v_{r-1}$ are determined uniquely by $v_{2}^{\prime}, \ldots, v_{r-1}^{\prime}$ modulo $W_{1}$. One can check that thus obtained $(x, \mathbf{v})$ is contained in $\in X_{\mathbf{m}}^{0}$. It follows that each fibre $\sigma^{-1}(z)$ is smooth with dimension $\operatorname{dim} H+(r-2) m_{1}$. Hence (2.5.3) holds.

Let $B_{1}$ be a Borel subgorup of $G_{1}$ which is the stabilizer of the flag $\left(M_{k}\right)_{0 \leq k \leq m_{1}}$ in $G_{1}$, and $B_{2}$ a $\theta$-stable Borel subgroup of $G_{2}$ which is the stabilizer of the flag $\left(M_{m_{1}+1} / M_{m_{1}} \subset \cdots \subset M_{n} / M_{m_{1}}\right)$ in $G_{2}$. Put

$$
\widetilde{G}_{1}=\left\{\left(x, g B_{1}\right) \in G_{1} \times G_{1} / B_{1} \mid g^{-1} x g \in B_{1}\right\}
$$

and define the map $\pi^{1}: \widetilde{G}_{1} \rightarrow G_{1}$ by $\left(x, g B_{1}\right) \mapsto x$. Put $\widetilde{G}_{1}^{0}=\left(\pi^{1}\right)^{-1}\left(G_{1}^{0}\right)$, and let $\varphi^{1}: \widetilde{G}_{1}^{0} \rightarrow G_{1}^{0}$ be the restriction of $\pi^{1}$. We define $\widetilde{\mathcal{X}}^{\prime}$ as the subvariety of $G_{2}^{\iota \theta} \times \bar{V}_{0}^{r-2} \times G_{2}^{\theta} / B_{2}^{\theta}$ as in the case of $\mathcal{X}^{\prime}$, and let $\pi^{2}: \widetilde{\mathcal{X}}^{\prime} \rightarrow \mathcal{X}^{\prime}$ be the projection $\left(x, \mathbf{v}^{\prime}, g B_{2}^{\theta}\right) \mapsto\left(x, \mathbf{v}^{\prime}\right)$. We put $\widetilde{\mathcal{X}}_{\mathbf{m}^{\prime}}^{\prime+}=\left(\pi^{2}\right)^{-1}\left(\mathcal{X}_{\mathbf{m}^{\prime}}^{\prime 0}\right)$, and let $\pi_{\mathbf{m}^{\prime}}^{2}$, be the restriction of $\pi^{2}$ on $\widetilde{\mathcal{X}}_{\mathbf{m}^{\prime}}^{\prime+}$. We define a variety

$$
\begin{aligned}
\widetilde{\mathcal{Z}}_{\mathbf{m}}^{+}=\left\{\left(x, \mathbf{v}, g B^{\theta}, \phi_{1}, \phi_{2}\right) \mid\left(x, \mathbf{v}, g B^{\theta}\right) \in \widetilde{\mathcal{X}}_{\mathbf{m}}^{+},\right. \\
\left.\phi_{1}: W_{z} \underset{\rightarrow}{\rightarrow} V_{0}, \phi_{2}: W_{z}^{\perp} / W_{z} \underset{\rightarrow}{\rightarrow} \bar{V}_{0} \text { for } z=(x, \mathbf{v})\right\},
\end{aligned}
$$

and define a map $\widetilde{q}: \widetilde{\mathcal{Z}}_{\mathbf{m}}^{+} \rightarrow \widetilde{\mathcal{X}}_{\mathbf{m}}^{+}$by a natural projection. We define a map $\widetilde{\sigma}$ : $\widetilde{\mathcal{Z}}_{\mathbf{m}}^{+} \rightarrow \widetilde{G}_{1}^{0} \times \widetilde{\mathcal{X}}_{\mathbf{m}^{\prime}}^{\prime+}$ as follows; take $\left(x, \mathbf{v}, g B^{\theta}, \phi_{1}, \phi_{2}\right) \in \widetilde{\mathcal{Z}}_{\mathbf{m}}^{+}$. Since $z=(x, \mathbf{v}) \in$ $\mathcal{X}_{\mathbf{m}}^{0}, W_{z}$ coinicdes with $g\left(M_{m_{1}}\right)$. Let $g_{1} B_{1}$ be the element corresponding to the flag $\phi_{1}\left(g\left(M_{i}\right)\right)_{0 \leq i \leq m_{1}}$, and $g_{2} B_{2}^{\theta}$ be the element corresponding to the isotorpic flag $\phi_{2}\left(g\left(M_{i}\right) / g\left(M_{m_{1}}\right)\right)_{i \geq m_{1}}$. Then

$$
\begin{aligned}
& \tilde{\sigma}:\left(x, \mathbf{v}, g B^{\theta}, \phi_{1}, \phi_{2}\right) \mapsto \\
& \quad\left(\left(\phi_{1}\left(\left.x\right|_{W_{z}}\right) \phi_{1}^{-1}, g_{1} B_{1}\right),\left(\phi_{2}\left(\left.x\right|_{w_{z}^{\perp} / W_{z}}\right) \phi_{2}^{-1}, \phi_{2}(\overline{\mathbf{v}}), g_{2} B_{2}^{\theta}\right)\right) .
\end{aligned}
$$


We also define a map $\widetilde{\pi}_{\mathbf{m}}: \widetilde{\mathcal{Z}}_{\mathbf{m}}^{+} \rightarrow \mathcal{K}_{\mathbf{m}}$ by $\left(x, \mathbf{v}, g B^{\theta}, \phi_{1}, \phi_{2}\right) \mapsto\left(x, \mathbf{v}, \phi_{1}, \phi_{2}\right)$. Then we have the following commutative diagram.

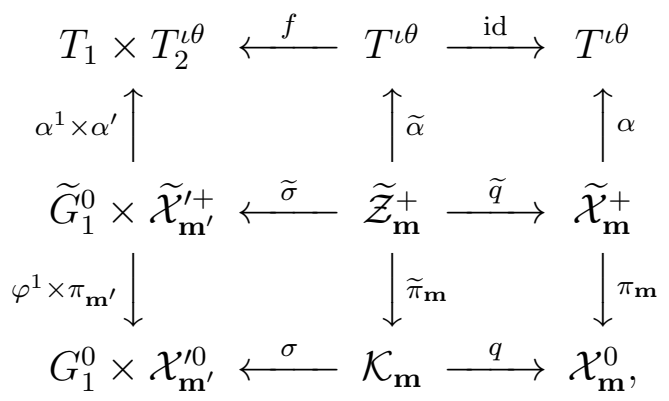

where the map $\widetilde{\alpha}$ is defined naturally. Note that $T^{\iota \theta}$ can be written as $T^{\iota \theta} \simeq T_{1} \times T_{2}^{\iota \theta}$, where $T_{1}$ is a maximal torus of $G_{1}$, and $T_{2}$ is a $\theta$-stable maximal torus of $G_{2}$. We fix an isomorphism $f: T^{\iota \theta} \rightarrow T_{1} \times T_{2}^{\iota \theta}$. The map $\alpha^{1}: \widetilde{G}_{1} \rightarrow T_{1}$ is defined as in 2.1, by ignoring $v$. The maps $\alpha^{\prime}, \pi_{\mathbf{m}^{\prime}}$ are defined similarly to $\alpha, \pi_{\mathbf{m}}$.

2.6. Let $T$ be a tame local system on $T^{\iota \theta}$. Under the isomorphism $f: T^{\iota \theta} \rightarrow$ $T_{1} \times T_{2}^{\iota \theta}, \mathcal{E}$ can be written as $\mathcal{E} \simeq \mathcal{E}_{1} \otimes \mathcal{E}_{2}$, where $\mathcal{E}_{1}$ (resp. $\mathcal{E}_{2}$ ) is a tame local system on $T_{1}$ (resp. $T_{2}^{\llcorner\theta}$ ). Then we have $\mathcal{W}_{\mathbf{m}, \mathcal{E}} \simeq \mathcal{W}_{1} \times \mathcal{W}_{\mathbf{m}^{\prime}, \mathcal{E}_{2}}^{\prime}$, where $\mathcal{W}_{1}$ is the stabilizer of $\mathcal{E}_{1}$ in $S_{m_{1}} \simeq N_{G_{1}}\left(T_{1}\right) / T_{1}$, and $\mathcal{W}_{\mathbf{m}^{\prime}, \mathcal{E}_{2}}^{\prime}$ is defined similarly to $\mathcal{W}_{\mathbf{m}, \mathcal{E}}$ with respect to $\mathcal{W}^{\prime}=N_{G_{2}^{\theta}}\left(T_{2}^{\iota \theta}\right) / Z_{G_{2}^{\theta}}\left(T_{2}^{\iota \theta}\right)$. As in 1.6, $\mathcal{W}_{\mathbf{m}, \mathcal{E}}$ is decomposed as $\mathcal{W}_{\mathbf{m}, \mathcal{E}} \simeq \mathcal{W}_{1} \times \cdots \times \mathcal{W}_{r}$ with subgroups $\mathcal{W}_{i} \subset S_{m_{i}}$. Then $\mathcal{W}_{\mathbf{m}^{\prime}, \mathcal{E}_{2}}^{\prime} \simeq \mathcal{W}_{2} \times \cdots \times \mathcal{W}_{r}$. For each $\rho \in \mathcal{W}_{\mathbf{m}, \mathcal{E}}^{\wedge}$, we construct a simple perverse sheaf $A_{\rho}$ on $\mathcal{X}_{\mathbf{m}}^{0}$ as follows; The decomposition of the complex $\pi_{!}^{1}\left(\alpha^{1}\right)^{*} \mathcal{E}_{1}\left[\operatorname{dim} G_{1}\right]$ into simple summands is well-known. Since $G_{1}^{0}$ is an open dense subset of $G_{1}$ containing $\left(G_{1}\right)_{\text {reg }}$, the decompostion of $\varphi_{!}^{1} \alpha^{*} \mathcal{E}_{1}$ is described similarly, namely we have

$$
\varphi_{!}^{1}\left(\alpha^{1}\right)^{*} \mathcal{E}_{1} \simeq \bigoplus_{\rho_{1} \in \mathcal{W}_{1}^{\wedge}} \rho_{1} \otimes \mathrm{IC}\left(G_{1}^{0}, \mathcal{L}_{\rho_{1}}\right)
$$

where $\mathcal{L}_{\rho_{1}}$ is a simple local system on the set of regular semisimple elements in $G_{1}$. Write $\rho$ as $\rho=\rho_{1} \otimes \cdots \otimes \rho_{r}$ with $\rho_{i} \in \mathcal{W}_{i}^{\wedge}$. Then $\rho^{\prime}=\rho_{2} \otimes \cdots \otimes \rho_{r} \in$ $\mathcal{W}_{\mathbf{m}^{\prime}, \mathcal{E}_{2}}^{\wedge}$. Suppose that a simple perverse sheaf $A_{\rho^{\prime}}$ on $\mathcal{X}_{\mathbf{m}^{\prime}}^{0}$ was constructed. Put $A_{1}=\mathrm{IC}\left(G_{1}^{0}, \mathcal{L}_{\rho_{1}}\right)\left[\operatorname{dim} G_{1}\right]$. Then $A_{1} \otimes A_{\rho^{\prime}}$ is an $H_{0^{-}}$equivariant simple perverse sheaf on $G_{1}^{0} \times \mathcal{X}_{\mathbf{m}^{\prime}}^{\prime \prime}$, and so $\sigma^{*}\left(A_{1} \otimes A_{\rho^{\prime}}\right)\left[\beta_{1}\right]$ is an $H_{0^{-}}$equivariant simple perverse sheaf on $\mathcal{K}_{\mathbf{m}}$ by $(2.5 .3)$, where $\beta_{1}=\operatorname{dim} H+(r-2) m_{1}$. Since $q$ is a principal bundle with group $H_{0}$ by $(2.5 .2)$, there exists a unique simple perverse sheaf $A_{\rho}$ on $\mathcal{X}_{\mathbf{m}}^{0}$ such that

$$
q^{*} A_{\rho}\left[\beta_{2}\right] \simeq \sigma^{*}\left(A_{1} \otimes A_{\rho^{\prime}}\right)\left[\beta_{1}\right]
$$

where $\beta_{2}=\operatorname{dim} H_{0}$. Note that in the case where $r=2, A_{\rho}$ coincides with the simple perverse sheaf $A_{\rho}$ constructed in [SS1, 4.6].

Let $\mathcal{L}_{\rho}$ be a simple local system on $\mathcal{Y}_{\mathbf{m}}^{0}$ appeared in (1.5.3). Since $\mathcal{Y}_{\mathbf{m}}^{0}$ is an open dense smooth subset of $\mathcal{X}_{\mathbf{m}}^{0}$, one can consider the intersection cohomology $\mathrm{IC}\left(\mathcal{X}_{\mathbf{m}}^{0}, \mathcal{L}_{\rho}\right)$ on $\mathcal{X}_{\mathbf{m}}^{0}$. We have the following lemma. 
Lemma 2.7. $A_{\rho} \simeq \operatorname{IC}\left(\mathcal{X}_{\mathbf{m}}^{0}, \mathcal{L}_{\rho}\right)\left[d_{\mathbf{m}}\right]$.

Proof. In order to prove the lemma, it is enough to see that

$$
\left.\mathcal{H}^{-d_{\mathbf{m}}} A_{\rho}\right|_{\mathcal{Y}_{\mathbf{m}}^{0}} \simeq \mathcal{L}_{\rho}
$$

We consider the following commutative diagram.

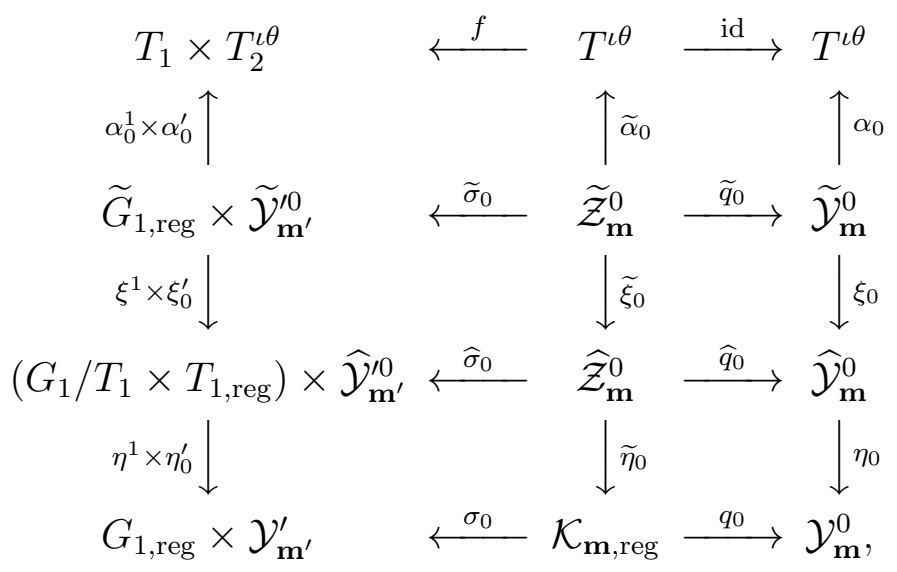

where $\widetilde{\mathcal{Y}}_{\mathbf{m}}^{0}=\widetilde{\mathcal{Y}}_{\mathbf{I}}, \widehat{\mathcal{Y}}_{\mathbf{m}}^{0}=\widehat{\mathcal{Y}}_{\mathbf{I}}$ for $\mathbf{I}=\mathbf{I}(\mathbf{m})$ (see 1.3), and $\widetilde{\mathcal{Y}}_{\mathbf{m}^{\prime}}^{\prime 0}=\widetilde{\mathcal{Y}}_{\mathbf{I}^{\prime}}^{\prime}, \widehat{\mathcal{Y}}_{\mathbf{m}^{\prime}}^{\prime 0}=\widehat{\mathcal{Y}}_{\mathbf{I}^{\prime}}^{\prime}$ are defined similarly with respect to $G_{2}^{\iota \theta} \times \bar{V}_{0}^{r-2}$ with $\mathbf{I}^{\prime}=\mathbf{I}\left(\mathbf{m}^{\prime}\right)$. Moreover,

$$
\begin{aligned}
\widetilde{G}_{1, \mathrm{reg}} & =\left(\pi^{1}\right)^{-1}\left(G_{1, \mathrm{reg}}\right), \\
\mathcal{K}_{\mathbf{m}, \mathrm{reg}} & =q^{-1}\left(\mathcal{Y}_{\mathbf{m}}^{0}\right), \\
\widetilde{\mathcal{Z}}_{\mathbf{m}}^{0} & =\widetilde{q}^{-1}\left(\widetilde{\mathcal{Y}}_{\mathbf{m}}^{0}\right)
\end{aligned}
$$

and $\widehat{\mathcal{Z}}_{\mathbf{m}}^{0}$ is defined as the quotient of $\widetilde{\mathcal{Z}}_{\mathbf{m}}^{0}$ under the natural action of $Z_{H}\left(T^{\iota \theta}\right)_{\mathbf{I}} /\left(B^{\theta} \cap\right.$ $\left.Z_{H}\left(T^{\iota \theta}\right)\right)$. The maps $\widetilde{q}_{0}, q_{0}, \widetilde{\sigma}_{0}, \sigma_{0}$ are defined as the restriction of the corresponding maps $\widetilde{q}, q, \widetilde{\sigma}, \sigma$. The map $\xi_{0}$ is $\xi_{\mathbf{I}}$ for $\mathbf{I}=\mathbf{I}(\mathbf{m})$. The maps $\widetilde{\xi}_{0}, \widetilde{\eta}_{0}$ are defined according to $\xi_{0}, \eta_{0} . \xi_{0}^{\prime}, \eta_{0}^{\prime}$ are defined similar to $\xi_{0}, \eta_{0}$ with respect to $G_{2}^{\iota \theta} \times \bar{V}_{0}^{r-2} . \xi^{1}, \eta^{1}$ are standard maps in the groups case ( $\xi^{1}$ is an isomorphism). The map $\widehat{\sigma}_{0}$ is naturally induced from $\widetilde{\sigma}_{0}$.

It follows from the diagram (2.7.2) that

$$
\widetilde{\sigma}_{0}^{*}\left(\left(\alpha_{0}^{1}\right)^{*} \mathcal{E}_{1} \otimes\left(\alpha_{0}^{2}\right)^{*} \mathcal{E}_{2}\right) \simeq \widetilde{q}_{0}^{*} \alpha_{0}^{*} \mathcal{E} .
$$

It is easy to check that the squares in the middle row and in the bottom row are all cartesian squares. Here $\psi^{1}=\eta^{1} \circ \xi^{1}: \widetilde{G}_{1, \text { reg }} \rightarrow G_{1, \text { reg }}$ is a finite Galois covering with group $S_{m_{1}}$, and $\psi_{!}^{1}\left(\alpha_{0}^{1}\right)^{*} \mathcal{E}_{1}$ is decomposed as

$$
\psi_{!}^{1}\left(\alpha_{0}^{1}\right)^{*} \mathcal{E}_{1} \simeq \bigoplus_{\rho_{1} \in \mathcal{W}_{1}^{\wedge}} \rho_{1} \otimes \mathcal{L}_{\rho_{1}} .
$$


On the other hand, by (1.5.3) and (1.6.3), we have

$$
\left(\eta_{0}^{\prime} \circ \xi_{0}^{\prime}\right)_{!}\left(\alpha_{0}^{2}\right)^{*} \mathcal{E}_{2} \simeq \bigoplus_{\rho^{\prime} \in\left(\mathcal{W}_{\mathbf{m}^{\prime}, \mathcal{E}_{2}}^{\prime}\right)^{\wedge}} H^{\bullet}\left(\mathbf{P}_{1}^{m_{r}}\right) \otimes \rho^{\prime} \otimes \mathcal{L}_{\rho^{\prime}}
$$

Similarly, the map $\eta_{0} \circ \xi_{0}$ coincides with $\psi_{\mathbf{I}}: \widetilde{\mathcal{Y}}_{\mathbf{I}}=\widetilde{\mathcal{Y}}_{\mathbf{m}}^{0} \rightarrow \mathcal{Y}_{\mathbf{m}}^{0}$. Hence we have

$$
\left(\eta_{0} \circ \xi_{0}\right) ! \alpha_{0}^{*} \mathcal{E} \simeq \bigoplus_{\rho \in \mathcal{W}_{\mathbf{m}, \mathcal{E}}^{\wedge}} H^{\bullet}\left(\mathbf{P}_{1}^{m_{r}}\right) \otimes \rho \otimes \mathcal{L}_{\rho}
$$

Simce the Galois covering is compatible with $\sigma_{0}$ and $q_{0}$ thanks to the diagram (2.7.2) (it corresponds to the squares in the bottom row), we have $\sigma_{0}^{*}\left(\mathcal{L}_{\rho_{1}} \otimes \mathcal{L}_{\rho^{\prime}}\right) \simeq q_{0}^{*} \mathcal{L}_{\rho}$ under the identification $\rho=\rho_{1} \otimes \rho^{\prime}$ for $\mathcal{W}_{\mathbf{m}, \mathcal{E}}=\mathcal{W}_{1} \times \mathcal{W}_{\mathbf{m}^{\prime}, \mathcal{E}_{2}}^{\prime}$. This implies that $\left.A_{\rho}\right|_{\mathcal{Y}_{\mathbf{m}}^{0}} \simeq \mathcal{L}_{\rho}\left[d_{\mathbf{m}}\right]$. Hence (2.7.1) holds and the lemma follows.

By using Lemma 2.7, we show the following.

Proposition 2.8. Under the notation in Lemma 2.7, $\left(\pi_{\mathbf{m}}\right) ! \alpha^{*} \mathcal{E}$ is decomposed as

$$
\left(\pi_{\mathbf{m}}\right) ! \alpha^{*} \mathcal{E} \simeq H^{\bullet}\left(\mathbf{P}_{1}^{m_{r}}\right) \otimes \bigoplus_{\rho \in \mathcal{W}_{\mathbf{m}, \mathcal{E}}^{\wedge}} \widetilde{V}_{\rho} \otimes \operatorname{IC}\left(\mathcal{X}_{\mathbf{m}}^{0}, \mathcal{L}_{\rho}\right)
$$

where $\widetilde{V}_{\rho}$ is regarded as a vector space, ignoring the $\widetilde{\mathcal{W}}_{\mathcal{E}}$-action.

Proof. We prove the proposition by induction on $r$. In the case where $r=2$, the proposition holds by Proposition 4.8 in [SS1]. We assume that the proposition holds for $r^{\prime}<r$. We fix $\mathbf{I} \in \mathcal{I}(\mathbf{m})$. Put $\widetilde{\mathcal{Z}}_{\mathbf{I}}=\widetilde{q}^{-1}\left(\widetilde{\mathcal{X}}_{\mathbf{I}}\right)$. We have the following commutative diagram

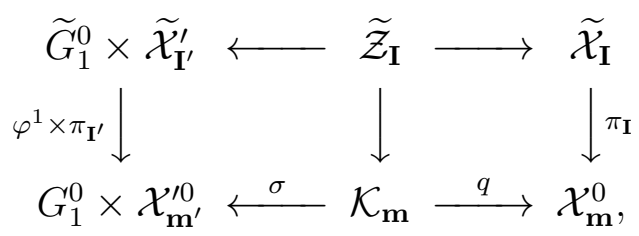

where $\pi_{\mathbf{I}}$ is the restriction of $\pi_{\mathbf{m}}$ on $\widetilde{\mathcal{X}}_{\mathbf{I}}$, and similarly for $\pi_{\mathbf{I}^{\prime}}$. Other maps are determined correspondingly. We note that both squares are cartesian squares.

We show the following.

(2.8.2) Any simple summand (up to shift) of the semisimple complex $\left(\pi_{\mathbf{I}}\right) ! \alpha^{*} \mathcal{E}$ is contained in the set $\left\{A_{\rho} \mid \rho \in \mathcal{W}_{\mathbf{m}, \mathcal{E}}^{\wedge}\right\}$.

Put $K_{1}=\varphi_{!}^{1}\left(\alpha^{1}\right)^{*} \mathcal{E}_{1}$, and $K_{2}=\left(\pi_{\mathbf{m}^{\prime}}\right) !\left(\alpha^{\prime}\right)^{*} \mathcal{E}_{2}$. By (2.6.1) and induction hypothesis, we have

$$
K_{1} \simeq \bigoplus_{\rho_{1} \in \mathcal{W}_{1}^{\wedge}} \rho_{1} \otimes \operatorname{IC}\left(G_{1}^{0}, \mathcal{L}_{\rho_{1}}\right)
$$




$$
K_{2} \simeq H^{\bullet}\left(\mathbf{P}_{1}^{m_{r}}\right) \otimes \bigoplus_{\rho^{\prime} \in \mathcal{W}_{\mathbf{m}^{\prime}, \mathcal{E}_{2}}^{\wedge}} \widetilde{V}_{\rho^{\prime}} \otimes \operatorname{IC}\left(\mathcal{X}_{\mathbf{m}^{\prime}}^{\prime 0}, \mathcal{L}_{\rho^{\prime}}\right)
$$

Put $K_{\mathbf{I}^{\prime}}=\left(\pi_{\mathbf{I}^{\prime}}\right)_{!}\left(\alpha^{\prime}\right)^{*} \mathcal{E}$. Since $\widetilde{\mathcal{X}}_{\mathbf{I}^{\prime}}^{\prime}$ is a connected component of $\widetilde{\mathcal{X}}_{\mathbf{m}^{\prime}}^{\prime+}$, any simple summand of $K_{\mathbf{I}^{\prime}}$ is contained in $K_{2}$, up to shift, thus it is of the form $\operatorname{IC}\left(\mathcal{X}_{\mathbf{m}^{\prime}}^{\prime 0}, \mathcal{L}_{\rho^{\prime}}\right)$. A simple perverse sheaf on $\mathcal{X}_{\mathbf{m}}^{0}$ obtained from $\operatorname{IC}\left(G_{1}^{0}, \mathcal{L}_{\rho_{1}}\right)$ and $\operatorname{IC}\left(\mathcal{X}_{\mathbf{m}^{\prime}}^{\prime 0}, \mathcal{L}_{\rho^{\prime}}\right)$ by the procedure in 2.6 actually coincides with $A_{\rho}$. On the other hand, since the squares in the diagram (2.8.1) are both cartesian, we have $\sigma_{1}^{*}\left(K_{1} \otimes K_{\mathbf{I}^{\prime}}\right) \simeq q_{1}^{*}\left(\left(\pi_{\mathbf{I}}\right)_{!} \alpha^{*} \mathcal{E}\right)$. (2.8.2) follows from this.

By Lemma 2.4 and Lemma 2.7, (2.8.2) implies that

(2.8.3) Any simple summand (up to shift) of the semisimple complex $\left(\pi_{\mathbf{m}}\right) ! \alpha^{*} \mathcal{E}$ is contained in the set $\left\{\operatorname{IC}\left(\mathcal{X}_{\mathbf{m}}^{0}, \mathcal{L}_{\rho}\right) \mid \rho \in \mathcal{W}_{\mathbf{m}, \mathcal{E}}^{\wedge}\right\}$.

(2.8.3) implies, in particular, that any simple summand of $K=\left(\pi_{\mathbf{m}}\right) ! \alpha^{*} \mathcal{E}$ has its support $\mathcal{X}_{\mathbf{m}}^{0}$. Since the restriction of $K$ on $\mathcal{Y}_{\mathbf{m}}^{0}$ coincides with $K_{0}=\left(\psi_{\mathbf{m}}\right) ! \alpha_{0}^{*} \mathcal{E}$, the decompostion of $K$ into simple summands is determined by the decomposition of $K_{0}$. Hence the proposition follows from (1.6.5).

Remark 2.9. Proposition 2.8 is a generalization of Proposition 4.8 in [SS1]. But the argument here is much simpler than that of [SS1]. Note that $A_{\rho}^{0}$ in $[\mathrm{SS} 1$, Lemma 4.7] corresponds to $A_{\rho}$ in Lemma 2.7 .

2.10. For $\mathbf{m} \in \mathcal{Q}_{n, r}$, and for each $j, k$, we consider $M^{(j, k)}$ and $\bar{M}^{(j, k)}$ as in the proof of Proposition 1.7. Put

$$
\begin{aligned}
& \mathcal{X}_{j, k}^{0}=\bigcup_{g \in H} g\left(B^{\iota \theta} \times M^{(j, k)}\right), \\
& \widetilde{\mathcal{X}}_{j, k}^{+}=\pi^{-1}\left(\mathcal{X}_{j, k}^{0}\right),
\end{aligned}
$$

and let $\pi_{j, k}: \widetilde{\mathcal{X}}_{j, k}^{+} \rightarrow \mathcal{X}_{j, k}^{0}$ be the restriction of $\pi$ on $\widetilde{\mathcal{X}}_{j, k}^{+}$. Then $\pi_{j, k}$ is a proper map, and $\mathcal{Y}_{j, k}^{0}$ is open dense in $\mathcal{X}_{j, k}^{0}$. Moreover $\mathcal{X}_{j, k}^{0}$ coincides with $\mathcal{X}_{\mathbf{m}}^{0}$ in the case where $j=r-1, k=m_{r-1}$, coincides with $\mathcal{X}_{\mathbf{m}}$ in the case where $j=0$. Also put

$$
\begin{aligned}
& \mathcal{X}_{j, k}=\bigcup_{g \in H} g\left(B^{\iota \theta} \times \bar{M}^{(j, k)}\right), \\
& \overline{\widetilde{\mathcal{X}}_{j, k}^{+}}=\pi^{-1}\left(\mathcal{X}_{j, k}\right),
\end{aligned}
$$

and let $\bar{\pi}_{j, k}: \overline{\widetilde{\mathcal{X}}_{j, k}^{+}} \rightarrow \mathcal{X}_{j, k}$ be the restriction of $\pi$ on $\overline{\widetilde{\mathcal{X}}_{j, k}^{+}}$. Then $\mathcal{X}_{j, k}^{0}$ is open dense in $\mathcal{X}_{j, k}$. As in (1.7.1), we have

(2.10.1) $\mathcal{X}_{j, k} \backslash \mathcal{X}_{j, k-1}=\mathcal{X}_{j, k}^{0}$ if $k \geq 1$, and $\mathcal{X}_{j, 0}(\mathbf{m})=\mathcal{X}_{j+1, m_{j}+m_{j+1}}(\mathbf{m}(j, 0))$. Moreover, $\mathcal{X}_{j, k}^{0}(\mathbf{m})$ coincides with $\mathcal{X}_{j+1, m_{j+1}}(\mathbf{m}(j, k))$.

For $\mathbf{m}^{\prime} \in \mathcal{Q}(\mathbf{m} ; j, k), \mathcal{Y}_{\mathbf{m}^{\prime}}^{0}$ is containd in $\mathcal{Y}_{j, k}$ hence in $\mathcal{X}_{j, k}$. One can define an intersection cohomology $\operatorname{IC}\left(\mathcal{X}_{\mathbf{m}^{\prime}}^{\prime}, \mathcal{L}_{\rho}\right)$ assoicated to the local sysmte $\mathcal{L}_{\rho}$ on $\mathcal{Y}_{\mathbf{m}^{\prime}}^{0}$ (here 
$\mathcal{X}_{\mathbf{m}^{\prime}}^{\prime}$ denotes the closure of $\mathcal{Y}_{\mathbf{m}^{\prime}}^{0}$ in $\left.\mathcal{X}_{j, k}\right)$. We show the following formulas. First assume that $j=r-1$ and $0 \leq k \leq m_{r-1}$.

$$
\begin{aligned}
\left(\bar{\pi}_{r-1, k}\right) ! \alpha^{*} \mathcal{E} & \\
& \simeq \bigoplus_{0 \leq k^{\prime} \leq k} \bigoplus_{\rho \in \mathcal{W}_{\mathbf{m}\left(k^{\prime}\right), \mathcal{E}}^{\wedge}} \widetilde{V}_{\rho} \otimes \mathrm{IC}\left(\mathcal{X}_{r-1, k^{\prime}}, \mathcal{L}_{\rho}\right)\left[-2\left(m_{r-1}-k^{\prime}\right)\right]+\mathcal{M}_{r-1, k}
\end{aligned}
$$

where $\mathcal{M}_{r-1, k}$ is a sum of various $\operatorname{IC}\left(\mathcal{X}_{r-1, k^{\prime}}, \mathcal{L}_{\rho}\right)[-2 i]$ for $0 \leq k^{\prime} \leq k$ and $\rho \in \mathcal{W}_{\mathbf{m}\left(k^{\prime}\right), \mathcal{E}}^{\wedge}$ with $0 \leq i<m_{r-1}-k^{\prime}$. Next assume that $0 \leq j<r-1$ and that $0 \leq k \leq m_{j}$. Then we have

$$
\begin{aligned}
& \left(\bar{\pi}_{j, k}\right) ! \alpha^{*} \mathcal{E} \\
& \quad \simeq \bigoplus_{0 \leq k^{\prime} \leq m_{r-1}} \bigoplus_{\rho \in \mathcal{W}_{\mathbf{m}\left(k^{\prime}\right), \mathcal{E}}^{\wedge}} \tilde{V}_{\rho} \otimes \operatorname{IC}\left(\mathcal{X}_{\mathbf{m}\left(k^{\prime}\right)}^{\prime}, \mathcal{L}_{\rho}\right)\left[-2\left(m_{r-1}-k^{\prime}\right)\right]+\mathcal{M}_{j, k}
\end{aligned}
$$

where $\mathcal{M}_{j, k}$ is a sum of various $\operatorname{IC}\left(\mathcal{X}_{\mathbf{m}^{\prime}}^{\prime}, \mathcal{L}_{\rho}\right)[-2 i]$ for $\mathbf{m}^{\prime} \in \mathcal{Q}(\mathbf{m} ; j, k)$ and $\rho \in \mathcal{W}_{\mathbf{m}^{\prime}, \mathcal{E}}^{\wedge}$ with $i$ such that $0 \leq 2 i<d_{\mathbf{m}}-d_{\mathbf{m}^{\prime}}$.

As in the proof of Proposition 1.7, one can define an action of $\widetilde{\mathcal{W}}_{\mathcal{E}}$ on $\left(\bar{\pi}_{j, k}\right) ! \alpha^{*} \mathcal{E}$. Then (2.10.2) and (2.10.3) can be proved by a similar argument as in the proof of Proposition 1.7.

Now apply $(2.10 .3)$ to the case where $j=0, k=0$. In this case, $\bar{\pi}_{j, k}$ coincides with $\bar{\pi}_{\mathbf{m}}$. (2.10.3) shows that any simple perverse sheaf $A[s]$ appearing in the semisimple complex $\left(\bar{\pi}_{\mathbf{m}}\right)_{!} \alpha^{*} \mathcal{E}$ has the property that $\operatorname{supp} A \cap \mathcal{Y}_{\mathbf{m}} \neq \emptyset . \mathcal{Y}_{\mathbf{m}}$ is open dense in $\mathcal{X}_{\mathbf{m}}$, and the restriction of $\left(\bar{\pi}_{\mathbf{m}}\right) ! \alpha^{*} \mathcal{E}$ on $\mathcal{Y}_{\mathbf{m}}$ coincides with $\left(\bar{\psi}_{\mathbf{m}}\right) ! \alpha_{0}^{*} \mathcal{E}$. Thus the theorem follows from Proposition 1.7. This complets the proof of Theorem 2.2.

\section{A variant of Theorem 2.2}

3.1. In this section, we assume that $\mathcal{X}$ is of exotic type. We keep the notation in Section 1 and Section 2. For $\mathbf{m}=\left(m_{1}, \ldots, m_{r-1}, 0\right) \in \mathcal{Q}_{n, r}^{0}$, put $\mathcal{W}_{\mathbf{m}}^{\natural}=S_{m_{1}} \times$ $\cdots \times S_{m_{r-2}} \times W_{m_{r-1}}$, where $W_{n}$ is the Weyl group of type $C_{n}$, and let $\mathcal{W}_{\mathbf{m}, \mathcal{E}}^{\natural}$ be the stabilizer of $\mathcal{E}$ in $\mathcal{W}_{\mathbf{m}}^{\natural}$. (Note that $\mathcal{W}_{\mathbf{m}}^{\natural}$ is not a subgroup of $W_{n, r}$ if $r \geq 3$.) Recall that $\mathbf{m}(k)=\left(m_{1}, \ldots, m_{r-2}, k, k^{\prime}\right)$ with $k+k^{\prime}=m_{r-1}$ for $\mathbf{m} \in \mathcal{Q}_{n, r}^{0}$. Hence $\mathcal{W}_{\mathbf{m}(k)} \simeq S_{m_{1}} \times \cdots \times S_{m_{r-2}} \times S_{k} \times S_{k^{\prime}}$. For $\rho=\rho_{1} \otimes \cdots \otimes \rho_{r} \in \mathcal{W}_{\mathbf{m}(k), \mathcal{E}}^{\wedge}$, we define an irreducible $\mathcal{W}_{\mathbf{m}, \mathcal{E}^{-}}^{\natural}$ module $V_{\rho}^{\natural}$ by $V_{\rho}^{\natural}=\rho_{1} \otimes \cdots \otimes \rho_{r-2} \otimes \widetilde{\rho}_{r-1}$, where $\widetilde{\rho}_{r-1}$ is an irreducible $W_{m_{r-1}}$-module obtained from $\rho_{r-1} \otimes \rho_{r} \in\left(S_{k} \times S_{k^{\prime}}\right)^{\wedge}$ (apply 1.6 for the case $r=2$ ). Recall the map $\pi^{(\mathbf{m})}: \widetilde{\mathcal{X}}_{\mathbf{m}} \rightarrow \mathcal{X}_{\mathbf{m}}$ as in 1.2 and consider $\left.\alpha\right|_{\widetilde{\mathcal{X}}_{\mathbf{m}}}: \widetilde{\mathcal{X}}_{\mathbf{m}} \rightarrow T^{\iota \theta}$, which we denote by the same symbol $\alpha$. The following result is a variant of Theorem 2.2 . 
Theorem 3.2. For each $\mathbf{m} \in \mathcal{Q}_{n, r}^{0}, \pi_{!}^{(\mathbf{m})} \alpha^{*} \mathcal{E}\left[d_{\mathbf{m}}\right]$ is a semisimple perverse sheaf on $\mathcal{X}_{\mathbf{m}}$ equipped with $\mathcal{W}_{\mathbf{m}, \mathcal{E}}^{\natural}$ action, and is decomposed as

$$
\pi_{!}^{(\mathbf{m})} \alpha^{*} \mathcal{E}\left[d_{\mathbf{m}}\right] \simeq \bigoplus_{0 \leq k \leq m_{r-1}} \bigoplus_{\rho \in \mathcal{W}_{\mathbf{m}(k), \mathcal{E}}^{\wedge}} V_{\rho}^{\natural} \otimes \operatorname{IC}\left(\mathcal{X}_{\mathbf{m}(k)}, \mathcal{L}_{\rho}\right)\left[d_{\mathbf{m}(k)}\right]
$$

3.3. The theorem can be proved in a similar way as in the proof of Theorem 2.2. We will give an outline of the proof below. We follow the notation in 1.3. We fix $\mathbf{m} \in \mathcal{Q}_{n, r}^{0}$, and put $\pi^{(\mathbf{m})}=\pi^{\bullet}$. We also consider the map $\psi^{(\mathbf{m})}: \widetilde{\mathcal{Y}}_{\mathbf{m}} \rightarrow \mathcal{Y}_{\mathbf{m}}$, which we denote by $\psi^{\bullet}$. For each $\mathbf{m}^{\prime} \leq \mathbf{m}\left(\mathbf{m}^{\prime} \in \mathcal{Q}_{n, r}\right)$, put $\tilde{\mathcal{Y}}_{\mathbf{m}^{\prime}}^{\dagger}=\left(\psi^{\bullet}\right)^{-1}\left(\mathcal{Y}_{\mathbf{m}^{\prime}}^{0}\right)$. For each $\mathbf{I} \in \mathcal{I}\left(\mathbf{m}^{\prime}\right)$, the variety $\widetilde{\mathcal{Y}}_{\mathbf{I}} \subset \widetilde{\mathcal{Y}}_{\mathbf{m}^{\prime}}^{\dagger}$ is defined as in 1.3. Put $\mathbf{I}^{\bullet}=\mathbf{I}(\mathbf{m}) \in \mathcal{I}(\mathbf{m})$, namely, $\mathbf{I}^{\bullet}=\left(I_{1}^{\circ}, \ldots, I_{r}^{\circ}\right)$ with $I_{i}^{\circ}=\left[p_{i-1}+1, p_{i}\right]$. In particular, $I_{r}^{\circ}=\emptyset$. Put $\mathcal{W}^{\bullet}=S_{m_{1}} \times \cdots \times S_{m_{r-1}}\left(=\mathcal{W}_{\mathbf{m}}\right)$, and we denote by $\mathcal{W}_{\mathbf{m}^{\prime}}^{\bullet}$ the subgroup of $\mathcal{W}^{\bullet}$ which is the stabilizer of $\mathbf{I}\left(\mathbf{m}^{\prime}\right)$. Put

$$
\mathcal{I}^{\bullet}\left(\mathbf{m}^{\prime}\right)=\left\{\mathbf{I} \in \mathcal{I}\left(\mathbf{m}^{\prime}\right) \mid I_{\leq i} \subset I_{\leq i}^{\circ}(1 \leq i \leq r)\right\}
$$

where $I_{\leq i}$ is defined similarly to $I_{<i}$ in 1.3 . Then as in (1.3.2), $\mathcal{W}^{\bullet}$ acts naturally on $\widetilde{\mathcal{Y}}_{\mathbf{m}^{\prime}}^{\dagger}$, and

$$
\widetilde{\mathcal{Y}}_{\mathbf{m}^{\prime}}^{\dagger}=\coprod_{\mathbf{I} \in \mathcal{I} \bullet\left(\mathbf{m}^{\prime}\right)} \widetilde{\mathcal{Y}}_{\mathbf{I}}=\coprod_{w \in \mathcal{W} \bullet / \mathcal{W}_{\mathbf{m}^{\prime}}^{\bullet}} w\left(\widetilde{\mathcal{Y}}_{\mathbf{m}^{\prime}}^{0}\right)
$$

where $\mathcal{Y}_{\mathbf{m}^{\prime}}^{0}=\mathcal{Y}_{\mathbf{I}\left(\mathbf{m}^{\prime}\right)}$ is as in 1.3. For a tame local system $\mathcal{E}$ on $T^{\iota \theta}$, we denote by $\mathcal{W}_{\mathcal{E}}^{\bullet}\left(\right.$ resp. $\mathcal{W}_{\mathbf{m}^{\prime}, \mathcal{E}}^{\bullet}$ ) the stabilizer of $\mathcal{E}$ in $\mathcal{W}^{\bullet}$ (resp. in $\mathcal{W}_{\mathbf{m}^{\prime}}^{\bullet}$ ). Put $\widetilde{\mathcal{W}}^{\bullet}=\mathcal{W}^{\bullet} \ltimes$ $(\mathbf{Z} / 2 \mathbf{Z})^{m_{r-1}}$, which coincides with $\mathcal{W}_{\mathbf{m}}^{\natural}$. We define a subgroup $\widetilde{\mathcal{W}_{\mathcal{E}}^{\bullet}}$ of $\widetilde{\mathcal{W}^{\bullet}}$ by $\widetilde{\mathcal{W}}_{\mathcal{E}}^{\bullet}=$ $\mathcal{W}_{\mathcal{E}}^{\bullet} \ltimes(\mathbf{Z} / 2 \mathbf{Z})^{m_{r-1}}$. Thus $\widetilde{\mathcal{W}}_{\mathcal{E}}^{\bullet}=\mathcal{W}_{\mathbf{m}, \mathcal{E}}^{\natural}$ in the notation of 3.1. Let $\psi_{\mathbf{m}^{\prime}}^{\bullet}$ be the restriction of $\psi^{\bullet}$ on $\widetilde{\mathcal{Y}}_{\mathbf{m}^{\prime}}^{\dagger}$. As an analogue of (1.5.2), we have

$$
\left.\left.\left(\psi_{\mathbf{m}^{\prime}}^{\bullet}\right) ! \alpha_{0}^{*} \mathcal{E}\right|_{\widetilde{\mathcal{Y}}_{\mathbf{m}^{\prime}}^{\dagger}} \simeq \bigoplus_{\mathbf{I} \in \mathcal{I}^{\bullet}\left(\mathbf{m}^{\prime}\right)}\left(\psi_{\mathbf{I}}\right) ! \alpha_{0}^{*} \mathcal{E}\right|_{\widetilde{\mathcal{Y}}_{\mathbf{I}}}
$$

Put $\mathbf{m}^{\prime}=\left(m_{1}^{\prime}, \ldots, m_{r}^{\prime}\right)$. The action of $(\mathbf{Z} / 2 \mathbf{Z})^{m_{r}^{\prime}}$ on $H^{\bullet}\left(\mathbf{P}_{1}^{m_{r}^{\prime}}\right)$ is defined as in 1.6 by considering the case where $r=2$. By a similar argument as in 1.5 and 1.6, we see that $\left(\psi_{\mathbf{m}^{\prime}}^{\bullet}\right) ! \alpha_{0}^{*} \mathcal{E}$ is equipped with $(\mathbf{Z} / 2 \mathbf{Z})^{m_{r}^{\prime}} \times \mathcal{W}_{\mathcal{E}^{-a c t i o n}}^{\bullet}$ and is decomposed as

$$
\left.\left(\psi_{\mathbf{m}^{\prime}}^{\bullet}\right) ! \alpha_{0}^{*} \mathcal{E}\right|_{\tilde{\mathcal{Y}}_{\mathbf{m}^{\prime}}^{\dagger}} \simeq H^{\bullet}\left(\mathbf{P}_{1}^{m_{r}^{\prime}}\right) \otimes \bigoplus_{\rho \in\left(\mathcal{W}_{\mathbf{m}^{\prime}, \mathcal{E}}^{\bullet}\right)^{\wedge}} \operatorname{Ind}_{\mathcal{W}_{\mathbf{m}^{\prime}, \mathcal{E}}^{\mathcal{W}_{\mathcal{E}}^{\bullet}}}^{\mathcal{E}^{\bullet}} \rho \otimes \mathcal{L}_{\rho}
$$

where $\mathcal{L}_{\rho}$ is a simple local system on $\mathcal{Y}_{\mathbf{m}^{\prime}}^{0}$ obtained from the Galois covering $\widehat{\mathcal{Y}}_{\mathbf{I}\left(\mathbf{m}^{\prime}\right)} \rightarrow$ $\mathcal{Y}_{\mathbf{m}^{\prime}}^{0}$ as in (1.5.3). 
As in (1.6.6), (3.3.3) can be rewritten in the following form;

$$
\left.\left(\psi_{\mathbf{m}^{\prime}}^{\bullet}\right) ! \alpha_{0}^{*} \mathcal{E}\right|_{\widetilde{\mathcal{Y}}_{\mathbf{m}^{\prime}}^{\dagger}} \simeq\left(\bigoplus_{\rho \in \mathcal{W}_{\mathbf{m}(k), \mathcal{E}}^{\wedge}} V_{\rho}^{\natural} \otimes \mathcal{L}_{\rho}\right)\left[-2 m_{r}^{\prime}\right]+\mathcal{N}_{\mathbf{m}^{\prime}}
$$

if $\mathbf{m}^{\prime}=\mathbf{m}(k)$ for some $k$, and $\left.\left(\psi_{\mathbf{m}^{\prime}}^{\bullet}\right) ! \alpha^{*} \mathcal{E}\right|_{\widetilde{\mathcal{Y}}_{\mathbf{m}^{\prime}}^{\dagger}} \simeq \mathcal{N}_{\mathbf{m}^{\prime}}$ otherwise, where $\mathcal{N}_{\mathbf{m}^{\prime}}$ is a sum of various $\mathcal{L}_{\rho}[-2 i]$ for $\rho \in\left(\mathcal{W}_{\mathbf{m}^{\prime}, \mathcal{E}}^{\bullet}\right)^{\wedge}$ with $2 i<d_{\mathbf{m}}-d_{\mathbf{m}^{\prime}}$.

In fact, put $\mathbf{p}\left(\mathbf{m}^{\prime}\right)=\left(p_{1}^{\prime}, \ldots, p_{r}^{\prime}\right)$. Since $\mathbf{m} \geq \mathbf{m}^{\prime}, p_{i} \geq p_{i}^{\prime}$ for each $i$. Moreover $p_{r-1}-p_{r-1}^{\prime}=m_{r}^{\prime}$ since $p_{r-1}=n$. Then by Lemma 1.4 we have

$$
\begin{aligned}
d_{\mathbf{m}}-d_{\mathbf{m}^{\prime}} & =\sum_{i=1}^{r-1}\left(p_{i}-p_{i}^{\prime}\right)+m_{r}^{\prime} \\
& =\sum_{i=1}^{r-2}\left(p_{i}-p_{i}^{\prime}\right)+2 m_{r}^{\prime} \\
& \geq 2 m_{r}^{\prime}
\end{aligned}
$$

and the equality holds only when $p_{i}=p_{i}^{\prime}$ for $i=1, \ldots, r-2$, namely when $\mathbf{m}^{\prime}=\mathbf{m}(k)$ for some $k$. By (3.3.3), $K=\left(\psi_{\mathbf{m}^{\prime}}^{\bullet}\right) ! \alpha_{0}^{*} \mathcal{E}$ is a semisimple complex and each direct summand is of the form $\mathcal{L}_{\rho}[-2 i]$ with $i \leq m_{r}^{\prime}$. Hence $K \simeq \mathcal{N}_{\mathbf{m}^{\prime}}$ if $\mathbf{m}^{\prime}$ is not of the form $\mathbf{m}(k)$. Now assume that $\mathbf{m}^{\prime}=\mathbf{m}(k)$. In this case, $\mathcal{W}_{\mathbf{m}^{\prime}, \mathcal{E}}^{\bullet}=\mathcal{W}_{\mathbf{m}(k), \mathcal{E}}$, and it follows from (3.3.3) that

$$
K \simeq \bigoplus_{\rho \in \mathcal{W}_{\mathbf{m}(k), \mathcal{E}}^{\wedge}} \operatorname{Ind}_{\widetilde{\mathcal{W}}_{\mathbf{m}(k), \mathcal{E}}^{\widetilde{\mathcal{W}}_{\dot{\mathcal{E}}}^{\bullet}}}\left(H^{\bullet}\left(\mathbf{P}_{1}^{m_{r}^{\prime}}\right) \otimes \rho\right) \otimes \mathcal{L}_{\rho}
$$

where $\widetilde{\mathcal{W}}_{\mathbf{m}(k), \mathcal{E}}^{\bullet}=\mathcal{W}_{\mathbf{m}(k), \mathcal{E}}^{\bullet} \ltimes(\mathbf{Z} / 2 \mathbf{Z})^{m_{r-1}}$, and $H^{\bullet}\left(\mathbf{P}_{1}^{m_{r}^{\prime}}\right) \otimes \rho$ is regarded as an $\widetilde{\mathcal{W}}_{\mathbf{m}(k), \mathcal{E}^{-}}^{\bullet}$ module by the trivial action of $(\mathbf{Z} / 2 \mathbf{Z})^{k}$, and by the action of $(\mathbf{Z} / 2 \mathbf{Z})^{m_{r}^{\prime}}$ through $H^{\bullet}\left(\mathbf{P}_{1}^{m_{r}^{\prime}}\right)$ (here $\left.k+m_{r}^{\prime}=m_{r-1}\right)$. The direct summand $\mathcal{L}_{\rho}[-2 i]$ of $K$ satisfies the relation $d_{\mathbf{m}}-d_{\mathbf{m}^{\prime}}=2 i$ only when $i=m_{r-1}^{\prime}$. Hence the first assertion of (3.3.4) follows from (3.3.5).

3.4. For $1 \leq j \leq r-1$ and $0 \leq k \leq m_{j}, M^{(j, k)}, \bar{M}^{(j, k)}, \mathcal{Y}_{j, k}^{0}, \mathcal{Y}_{j, k}$ are defined as in the proof of Proposition 1.7. Put $\widetilde{\mathcal{Y}}_{j, k}^{\dagger}=\left(\psi^{\bullet}\right)^{-1}\left(\mathcal{Y}_{j, k}^{0}\right)$ and $\frac{\widetilde{\mathcal{Y}}_{j, k}^{\dagger}}{\dagger}=\left(\psi^{\bullet}\right)^{-1}\left(\mathcal{Y}_{j, k}\right)$. Let $\psi_{j, k}^{\bullet}: \tilde{\mathcal{Y}}_{j, k}^{\dagger} \rightarrow \mathcal{Y}_{j, k}^{0}$ be the restriction of $\psi^{\bullet}$ on $\tilde{\mathcal{Y}}_{j, k}^{\dagger}$, and $\bar{\psi}_{j, k}^{\bullet}: \overline{\mathcal{Y}}_{j, k}^{\dagger} \rightarrow \mathcal{Y}_{j, k}$ the restriction of $\psi^{\bullet}$ on $\widetilde{\mathcal{Y}}_{j, k}^{\dagger}$. By using a similar argument as in the proof of (1.7.2) and (1.7.3), we can show the following formulas; first assume that $j=r-1$ and 
$0 \leq k \leq m_{r-1}$

$$
\begin{aligned}
& \left(\bar{\psi}_{r-1, k}^{\bullet}\right) ! \alpha_{0}^{*} \mathcal{E} \\
& \simeq \bigoplus_{0 \leq k^{\prime} \leq k} \bigoplus_{\rho \in \mathcal{W}_{\mathbf{m}\left(k^{\prime}\right), \mathcal{E}}^{\wedge}} V_{\rho}^{\natural} \otimes \operatorname{IC}\left(\mathcal{Y}_{r-1, k^{\prime}}, \mathcal{L}_{\rho}\right)\left[-2\left(m_{r-1}-k^{\prime}\right)\right]+\mathcal{N}_{r-1, k},
\end{aligned}
$$

where $\mathcal{N}_{r-1, k}$ is a sum of various $\operatorname{IC}\left(\mathcal{Y}_{r-1, k^{\prime}}, \mathcal{L}_{\rho}\right)[-2 i]$ for $0 \leq k^{\prime} \leq k$ and $\rho \in \mathcal{W}_{\mathbf{m}\left(k^{\prime}\right), \mathcal{E}}^{\wedge}$ with $i<m_{r-1}-k^{\prime}$. Next assume that $0 \leq j<r-1$ and that $0 \leq k \leq m_{j}$. Then we have

$$
\begin{aligned}
& \left(\bar{\psi}_{j, k}^{\bullet}\right)_{*} \alpha_{0}^{*} \mathcal{E} \\
& \quad \simeq \bigoplus_{0 \leq k^{\prime} \leq m_{r-1}} \bigoplus_{\rho \in \mathcal{W}_{\mathbf{m}\left(k^{\prime}\right), \mathcal{E}}^{\wedge}} V_{\rho}^{\natural} \otimes \operatorname{IC}\left(\mathcal{Y}_{\mathbf{m}\left(k^{\prime}\right)}^{\prime}, \mathcal{L}_{\rho}\right)\left[-2\left(m_{r-1}-k^{\prime}\right)\right]+\mathcal{N}_{j, k},
\end{aligned}
$$

where $\mathcal{N}_{j, k}$ is a sum of various $\operatorname{IC}\left(\mathcal{Y}_{\mathbf{m}^{\prime}}^{\prime}, \mathcal{L}_{\rho}\right)[-2 i]$ for $\mathbf{m}^{\prime} \in \mathcal{Q}(\mathbf{m} ; j, k)$ and $\rho \in\left(\mathcal{W}_{\mathbf{m}^{\prime}, \mathcal{E}}^{\bullet}\right)^{\wedge}$ with $i$ such that $2 i<d_{\mathbf{m}}-d_{\mathbf{m}^{\prime}}$.

Note that in the proof of $(3.4 .1)$, the role of the irreducible $\widetilde{\mathcal{W}}_{\mathcal{E}}$-module $\widetilde{V}_{\rho}$ is replaced by the irreducible $\mathcal{W}_{\mathbf{m}, \mathcal{E}^{\natural}}^{\natural}$ module $V_{\rho}^{\natural}$.

By a similar argument as in the proof of Lemma 1.4 (iv), one can show that $\psi^{(\mathbf{m})}$ is semismall for $\mathbf{m} \in \mathcal{Q}_{n, r}^{0}$. Then, as in the proof of Proposition 1.7 (see the paragraph after (1.7.3)), we obtain the following proposition from (3.4.2).

Proposition 3.5. For each $\mathbf{m} \in \mathcal{Q}_{n, r}^{0}, \psi_{!}^{(\mathbf{m})} \alpha_{0}^{*} \mathcal{E}\left[d_{\mathbf{m}}\right]$ is a semisimple perverse sheaf on $\mathcal{Y}_{\mathbf{m}}$ equipped with $\mathcal{W}_{\mathbf{m}, \mathcal{E}^{-}}$action, and is decomposed as

$$
\psi_{!}^{(\mathbf{m})} \alpha_{0}^{*} \mathcal{E}\left[d_{\mathbf{m}}\right] \simeq \bigoplus_{0 \leq k \leq m_{r-1}} \bigoplus_{\rho \in \mathcal{W}_{\mathbf{m}(k), \mathcal{E}}^{\wedge}} V_{\rho}^{\natural} \otimes \operatorname{IC}\left(\mathcal{Y}_{\mathbf{m}(k)}, \mathcal{L}_{\rho}\right)\left[d_{\mathbf{m}(k)}\right]
$$

3.6. We follow the notation in 2.3. For $\mathbf{m}^{\prime} \in \mathcal{Q}_{n, r}$ such that $\mathbf{m}^{\prime} \leq \mathbf{m}$, put $\widetilde{\mathcal{X}}_{\mathbf{m}^{\prime}}^{\dagger}=\left(\pi^{\bullet}\right)^{-1}\left(\mathcal{X}_{\mathbf{m}^{\prime}}^{0}\right)$. Then $\widetilde{\mathcal{Y}}_{\mathbf{m}^{\prime}}^{\dagger}$ is an open dense subset of $\widetilde{\mathcal{X}}_{\mathbf{m}^{\prime}}^{\dagger}$. For each $\mathbf{I} \in \mathcal{I}\left(\mathbf{m}^{\prime}\right)$, the subvariety $\widetilde{\mathcal{X}}_{\mathbf{I}}$ of $\widetilde{\mathcal{X}}_{\mathbf{m}^{\prime}}^{+}$is defined as in 2.3. Then as in Lemma 2.4, we have

$$
\widetilde{\mathcal{X}}_{\mathbf{m}^{\prime}}^{\dagger}=\coprod_{\mathbf{I} \in \mathcal{I}^{\bullet}\left(\mathbf{m}^{\prime}\right)} \widetilde{\mathcal{X}}_{\mathbf{I}}
$$

where $\widetilde{\mathcal{X}}_{\mathbf{I}}$ is an irreducible component of $\widetilde{\mathcal{X}}_{\mathbf{m}^{\prime}}^{\dagger}$.

Let $\pi_{\mathbf{m}^{\prime}}^{\bullet}: \widetilde{\mathcal{X}}_{\mathbf{m}^{\prime}}^{\dagger} \rightarrow \mathcal{X}_{\mathbf{m}^{\prime}}^{0}$ be the restriction of $\pi^{(\mathbf{m})}$ on $\widetilde{\mathcal{X}}_{\mathbf{m}^{\prime}}^{\dagger}$. The following result is an analogue of Proposition 2.8, and is proved in a similar way.

Proposition 3.7. Assume that $\mathbf{m}^{\prime} \leq \mathbf{m}$. Then $\left(\pi_{\mathbf{m}^{\prime}}^{\bullet}\right) ! \alpha^{*} \mathcal{E}$ is decomposed as

$$
\left(\pi_{\mathbf{m}^{\prime}}^{\bullet}\right) ! \alpha^{*} \mathcal{E} \simeq H^{\bullet}\left(\mathbf{P}_{1}^{m_{r}^{\prime}}\right) \otimes \bigoplus_{\rho \in \mathcal{W}_{\mathbf{m}^{\prime}, \mathcal{E}}^{\bullet \wedge}} \operatorname{Ind}_{\mathcal{W}_{\mathbf{m}^{\prime}, \mathcal{E}}^{\bullet}}^{\mathcal{W}_{\mathcal{\varepsilon}}^{\bullet}} \rho \otimes \operatorname{IC}\left(\mathcal{X}_{\mathbf{m}^{\prime}}^{0}, \mathcal{L}_{\rho}\right)
$$


where $\operatorname{Ind}_{\mathcal{W}_{\mathbf{m}^{\prime}, \mathcal{E}}^{\mathcal{W}_{\boldsymbol{E}}}}^{\mathcal{W}^{\prime}} \rho$ is regarded as a vector space ignoring the $\mathcal{W}_{\mathcal{E}}^{\bullet}$-module structure.

3.8. By making use of Proposition 3.5 and 3.7, the theorem can be proved in a similar way as in $2.9,2.10$.

\section{Intersetion COHOMOLOGY ON $G^{\iota \theta} \times V^{r-1}$ (ENHANCED CASE)}

4.1. In this section we assume that $\mathcal{X}$ is of enhanced type. We fix $\mathbf{m} \in \mathcal{Q}_{n, r}$ (note that we don't assume $\mathbf{m} \in \mathcal{Q}_{n, r}^{0}$ ), and consider the map $\pi^{(\mathbf{m})}: \widetilde{\mathcal{X}}_{\mathbf{m}} \rightarrow \mathcal{X}_{\mathbf{m}}$. Here in order to emphasize a similarlty with the exotic case, we follow the notation in Section 1. But of course a simpler expression is possible for the enhanced case. For example, if we write $G=G_{0} \times G_{0}$ and $B=B_{0} \times B_{0}$ for a Borel subgroup $B_{0}$ of $G_{0}, \widetilde{\mathcal{X}}_{\mathbf{m}}, \mathcal{X}_{\mathbf{m}}$ are given by

$$
\begin{aligned}
& \widetilde{\mathcal{X}}_{\mathbf{m}}=\left\{\left(x, \mathbf{v}, g B_{0}\right) \in G_{0} \times V^{r-1} \times G_{0} / B_{0} \mid g^{-1} x g \in B_{0}, g^{-1} \mathbf{v} \in \prod_{i=1}^{r-1} M_{p_{i}}\right\} \\
& \mathcal{X}_{\mathbf{m}}=\bigcup_{g \in G_{0}} g\left(B_{0} \times \prod_{i=1}^{r-1} M_{p_{i}}\right) .
\end{aligned}
$$

The map $\psi^{(\mathbf{m})}: \widetilde{\mathcal{Y}}_{\mathbf{m}} \rightarrow \mathcal{Y}_{\mathbf{m}}$ is defined as in 1.2. We put $\pi^{(\mathbf{m})}=\pi^{\bullet}$ and $\psi^{(\mathbf{m})}=\psi^{\bullet}$. The subset $\mathcal{Y}_{\mathbf{m}^{\prime}}^{0}$ is defined for each $\mathbf{m}^{\prime} \in \mathcal{Q}_{n, r}$ as in 1.3. For each $\mathbf{m}^{\prime} \leq \mathbf{m}$, put $\widetilde{\mathcal{Y}}_{\mathbf{m}^{\prime}}^{\dagger}=\left(\psi^{\bullet}\right)^{-1}\left(\mathcal{Y}_{\mathbf{m}^{\prime}}^{0}\right)$. For each $\mathbf{I} \in \mathcal{I}\left(\mathbf{m}^{\prime}\right)$, the subvariety $\widetilde{\mathcal{Y}}_{\mathbf{I}}$ of $\widetilde{\mathcal{Y}}_{\mathbf{m}^{\prime}}^{\dagger}$ and the map $\psi_{\mathbf{I}}$ are defined as in 1.3. Note that in the enhanced case, if we write $T=T_{0} \times T_{0}$, then $Z_{H}\left(T^{\iota \theta}\right)=Z_{G_{0}}\left(T_{0}\right)=T_{0}$. Hence $B^{\theta} \cap Z_{H}\left(T^{\llcorner\theta}\right)=T_{0}$, and $T_{\text {reg }}^{\iota \theta}$ is the set of regular semisimple elements in $T_{0}$. Hence $\widetilde{\mathcal{Y}}_{\mathbf{I}}$ is written as

$$
\widetilde{\mathcal{Y}}_{\mathbf{I}} \simeq G_{0} \times{ }^{T_{0}}\left(\left(T_{0}\right)_{\mathrm{reg}} \times M_{\mathbf{I}}\right)
$$

where $M_{\mathbf{I}}$ is defined as in 1.3. As in 3.3, we define $\mathcal{W}^{\bullet}=S_{m_{1}} \times \cdots \times S_{m_{r}}\left(=\mathcal{W}_{\mathbf{m}}\right)$, and its subgroup $\mathcal{W}_{\mathbf{m}^{\prime}}^{\bullet}$. For each $\mathbf{m}^{\prime} \leq \mathbf{m}$, we define $\mathcal{I}^{\bullet}\left(\mathbf{m}^{\prime}\right)$ as in 3.3. Then a simlar formula as (3.3.1) holds for $\widetilde{\mathcal{Y}}_{\mathbf{m}^{\prime}}^{\dagger}$. Let $\mathcal{E}$ be a tame local system on $T^{\iota \theta}$, and we denote by $\mathcal{W}_{\mathcal{E}}^{\bullet}$ (resp. $\mathcal{W}_{\mathbf{m}^{\prime}, \mathcal{E}}^{\bullet}$ ) the stabilizer of $\mathcal{E}$ in $\mathcal{W}^{\bullet}$ (resp. in $\mathcal{W}_{\mathbf{m}^{\prime}}^{\bullet}$ ). As in (1.5.2), we have a similar formula as (3.3.2). Note that in the enhanced case, one can check that $\psi_{\mathbf{I}}$ is a finite Galois covering with group $\mathcal{W}_{\mathbf{I}}^{\bullet}$ ( the stbilizer of $\mathbf{I}$ in $\mathcal{W}^{\bullet}$ ). It follws from (3.3.1) and (3.3.2) (corresponding formulas for the enhanced case) we have

$$
\left.\left(\psi_{\mathbf{m}^{\prime}}^{\bullet}\right) ! \alpha_{0}^{*} \mathcal{E}\right|_{\tilde{\mathcal{Y}}_{\mathbf{m}^{\prime}}^{\dagger}} \simeq \bigoplus_{\rho \in\left(\mathcal{W}_{\mathbf{m}^{\prime}, \mathcal{E}}^{\bullet}\right.}\left(\operatorname{Ind}_{\mathcal{W}_{\mathbf{m}^{\prime}, \mathcal{E}}^{\mathcal{W}_{\mathcal{E}}}}^{\mathcal{W}^{\bullet}} \rho\right) \otimes \mathcal{L}_{\rho}
$$

Here we note that 
Lemma 4.2. (i) $\mathcal{Y}_{\mathbf{m}}$ is open dense in $\mathcal{X}_{\mathbf{m}}$ and $\widetilde{\mathcal{Y}}_{\mathbf{m}}$ is open dense in $\widetilde{\mathcal{X}}_{\mathbf{m}}$

(ii) $\operatorname{dim} \mathcal{X}_{\mathbf{m}}=\operatorname{dim} \widetilde{\mathcal{X}}_{\mathbf{m}}=n^{2}+\sum_{i=1}^{r}(r-i) m_{i}$.

(iii) For any $(x, \mathbf{v}) \in \mathcal{Y}_{\mathbf{m}},\left(\psi^{(\mathbf{m})}\right)^{-1}(x, \mathbf{v})$ is a finite set.

Proof. $\widetilde{\mathcal{Y}}_{\mathbf{m}}$ is an open dense subset of $\widetilde{\mathcal{X}}_{\mathbf{m}}$. Since $\widetilde{\mathcal{Y}}_{\mathbf{m}}=\left(\pi^{(\mathbf{m})}\right)^{-1}\left(\mathcal{Y}_{\mathbf{m}}\right)$ and $\pi^{(\mathbf{m})}$ is proper, $\mathcal{Y}_{\mathbf{m}}$ is an open dense subset of $\mathcal{X}_{\mathbf{m}}$. Hence (i) holds. $\widetilde{\mathcal{Y}}_{\mathbf{m}}^{\dagger}$ is an open dense subset of $\widetilde{\mathcal{Y}}_{\mathbf{m}}$, and $\mathcal{Y}_{\mathbf{m}}^{0}$ is an open dense subset of $\mathcal{Y}_{\mathbf{m}}$. Since $\psi_{\mathbf{I}}$ is a finite Galois covering for $\mathbf{I} \in \mathcal{I}(\mathbf{m})$, we have $\operatorname{dim} \widetilde{\mathcal{Y}}_{\mathbf{m}}^{\dagger}=\operatorname{dim} \mathcal{Y}_{\mathbf{m}}^{0}$. Hence $\operatorname{dim} \widetilde{\mathcal{Y}}_{\mathbf{m}}=\operatorname{dim} \mathcal{Y}_{\mathbf{m}}$ and (ii) follows from (1.2.1). (iii) is clear since $\psi_{\mathbf{I}}$ is a finite Galois covering for any $\mathbf{I} \in \mathcal{I}^{\bullet}\left(\mathbf{m}^{\prime}\right)$.

Next we show the following proposition.

Proposition 4.3. For each $\mathbf{m} \in \mathcal{Q}_{n, r}, \psi_{!}^{(\mathbf{m})} \alpha_{0}^{*} \mathcal{E}\left[d_{\mathbf{m}}\right]$ is a semisimple perverse sheaf on $\mathcal{Y}_{\mathbf{m}}$ equipped with $\mathcal{W}_{\mathbf{m}, \mathcal{E}^{-}}^{\bullet}$ action, and is decomposed as

$$
\psi_{!}^{(\mathbf{m})} \alpha_{0}^{*} \mathcal{E}\left[d_{\mathbf{m}}\right] \simeq \bigoplus_{\rho \in \mathcal{W}_{\mathbf{m}, \mathcal{E}}^{\wedge}} \rho \otimes \operatorname{IC}\left(\mathcal{Y}_{\mathbf{m}}, \mathcal{L}_{\rho}\right)\left[d_{\mathbf{m}}\right]
$$

Proof. $\psi^{(\mathbf{m})}: \widetilde{\mathcal{Y}}^{(\mathbf{m})} \rightarrow \mathcal{Y}_{\mathbf{m}}$ is proper. By Lemma $4.2($ iii $), \psi^{(\mathbf{m})}$ is semismall. Hence $\psi_{!}^{(\mathbf{m})} \alpha_{0}^{*} \mathcal{E}\left[d_{\mathbf{m}}\right]$ is a semisimple perverse sheaf. The definitions of $\mathcal{Y}_{j, k}^{0}, \mathcal{Y}_{j, k}, \psi_{j, k}^{\bullet}, \bar{\psi}_{j, k}^{\bullet}$, etc. in 3.4 make sense also in the enhanced case. As in (3.4.1) and (3.4.2), the following formulas hold; first assume that $j=r-1,0 \leq k<m_{r-1}$. Then we have

$$
\left(\bar{\psi}_{j, k}^{\bullet}\right)_{!} \alpha_{0}^{*} \mathcal{E} \simeq \mathcal{N}_{r-1, k}
$$

Next assume that $j=r-1, k=m_{r-1}$ or $0 \leq j<r-1,0 \leq k \leq m_{j}$. Then we have

$$
\left(\bar{\psi}_{j, k}^{\bullet}\right)_{!} \alpha_{0}^{*} \mathcal{E} \simeq \bigoplus_{\rho \in \mathcal{W}_{\mathbf{m}, \mathcal{E}}^{\wedge}} \rho \otimes \operatorname{IC}\left(\mathcal{Y}_{\mathbf{m}}^{\prime}, \mathcal{L}_{\rho}\right)+\mathcal{N}_{j, k}
$$

where $\mathcal{N}_{j, k}$ is a sum of various $\operatorname{IC}\left(\mathcal{Y}_{\mathbf{m}^{\prime}}^{\prime}, \mathcal{L}_{\rho}\right)$ for $\mathbf{m}^{\prime} \in \mathcal{Q}(\mathbf{m} ; j, k)$ and $\rho \in\left(\mathcal{W}_{\mathbf{m}^{\prime}, \mathcal{E}}^{\bullet}\right)^{\wedge}$ such that $\mathbf{m}^{\prime}<\mathbf{m}$. (Recall that $\mathcal{Y}_{\mathbf{m}^{\prime}}^{\prime}$ denotes the closure of $\mathcal{Y}_{\mathbf{m}^{\prime}}^{0} \subset \mathcal{Y}_{j, k}$ in $\mathcal{Y}_{j, k}$ for any $\mathbf{m}^{\prime} \leq \mathbf{m}$.)

We show (4.3.1) by induction on $k$. By (1.7.1) (or directly from the definition of $\left.M^{(r-1, k)}\right), \mathcal{Y}_{r-1,0}(\mathbf{m})$ coincides with $\mathcal{Y}_{\mathbf{m}(0)}^{0}$. Hence by $(4.1 .1)$ for $\mathbf{m}^{\prime}=\mathbf{m}(0),(4.3 .1)$ holds for $k=0$. A similar argument as in the proof of (1.7.2) shows, thanks to (1.7.1) and (4.1.1), that (4.3.1) holds for $k<m_{r-1}$. Next consider the case where $j=r-1, k=m_{r-1}$. By $(1.7 .1), \mathcal{Y}_{j, k} \backslash \mathcal{Y}_{j, k-1}=\mathcal{Y}_{j, k}^{0}$, and $\mathcal{Y}_{j, k}^{0}$ coinicides with $\mathcal{Y}_{\mathbf{m}}^{0}$. Thus (4.1.1) implies that

$$
\left(\psi_{j, k}^{\bullet}\right) ! \alpha_{0}^{*} \mathcal{E} \simeq \bigoplus_{\rho \in \mathcal{W}_{\mathbf{m}, \mathcal{E}}^{\wedge}} \rho \otimes \mathcal{L}_{\rho}
$$

Hence (4.3.2) holds in this case. Now (4.3.2) can be proved by induction on $k$ and by backwards induction on $j$, starting from $j=r-2, k=0$, which case corresponds 
to the case where $j=r-1, k=m_{r-1}$ by (1.7.1). Note that in the enhanced case, we don't need a discussion such as (1.7.5).

Applying (4.3.2) to the case where $j=0, k=0$, we obtain the proposition. In fact, in that case, $\mathcal{N}_{0.0}$ is a sum of $A=\operatorname{IC}\left(\mathcal{Y}_{\mathbf{m}^{\prime}}, \mathcal{L}_{\rho}\right)$ such that $\mathbf{m}^{\prime}<\mathbf{m}$. But then $A\left[d_{\mathbf{m}}\right]$ is not a perverse sheaf. Since $\psi_{!}^{(\mathbf{m})} \alpha_{0}^{*} \mathcal{E}\left[d_{\mathbf{m}}\right]$ is a semisimple perverse sheaf, this implies that $\mathcal{N}_{0.0}=0$, and the proposition follows.

4.4. As a special case of (4.1.1) for $\mathbf{m}^{\prime}=\mathbf{m}$, we have

$$
\left(\psi_{\mathbf{m}}^{\bullet}\right) ! \alpha_{0}^{*} \mathcal{E} \simeq \bigoplus_{\rho \in \mathcal{W}_{\mathbf{m}, \mathcal{E}}^{\wedge}} \rho \otimes \mathcal{L}_{\rho}
$$

Since $\mathcal{Y}_{\mathbf{m}}^{0}$ is a smooth open dense subset of $\mathcal{X}_{\mathbf{m}}$, one can define a semisimple pervers sheaf $K_{\mathbf{m}, T, \mathcal{E}}$ on $\mathcal{X}_{\mathbf{m}}$ as

$$
K_{\mathbf{m}, T, \mathcal{E}}=\bigoplus_{\rho \in \mathcal{W}_{\mathbf{m}, \mathcal{E}}^{\wedge}} \rho \otimes \operatorname{IC}\left(\mathcal{X}_{\mathbf{m}}, \mathcal{L}_{\rho}\right)\left[d_{\mathbf{m}}\right]
$$

We consider a diagram

$$
T^{\iota \theta} \stackrel{\alpha}{\longleftarrow} \widetilde{\mathcal{X}}_{\mathbf{m}} \stackrel{\pi^{(\mathbf{m})}}{\longrightarrow} \mathcal{X}_{\mathbf{m}}
$$

where $\alpha$ is as in 2.1, and a complex $\pi_{!}^{(\mathbf{m})} \alpha^{*} \mathcal{E}\left[d_{\mathbf{m}}\right]$ on $\mathcal{X}_{\mathbf{m}}$. We shall prove the following theorem.

Theorem 4.5. For each $\mathbf{m} \in \mathcal{Q}_{n, r}, \pi_{!}^{(\mathbf{m})} \alpha^{*} \mathcal{E}\left[d_{\mathbf{m}}\right] \simeq K_{\mathbf{m}, T, \mathcal{E}}$ as perverse sheaves on $\mathcal{X}_{\mathrm{m}}$.

4.6. For each $\mathbf{m}^{\prime} \leq \mathbf{m}$, the set $\mathcal{X}_{\mathbf{m}^{\prime}}^{0}$ is defined as in 2.3. We define a subvariety $\mathcal{X}_{\mathbf{m}^{\prime}}^{\dagger}$ of $\widetilde{\mathcal{X}}_{\mathbf{m}}$ by $\widetilde{\mathcal{X}}_{\mathbf{m}^{\prime}}^{\dagger}=\left(\pi^{\bullet}\right)^{-1}\left(\mathcal{X}_{\mathbf{m}^{\prime}}^{0}\right)$. Then $\widetilde{\mathcal{Y}}_{\mathbf{m}^{\prime}}^{\dagger}$ is an open dense subset of $\widetilde{\mathcal{X}}_{\mathbf{m}^{\prime}}^{\dagger}$. The discussion in 2.3 makes sense also for the enhanced case, and $\mathcal{X}_{\mathbf{m}^{\prime}}^{0}$ is characterized by a similar formula as (2.3.3). For each $\mathbf{I} \in \mathcal{I}\left(\mathbf{m}^{\prime}\right)$, the set $\widetilde{\mathcal{X}}_{\mathbf{I}}$ is defined as in 2.3,

$$
\widetilde{\mathcal{X}}_{\mathbf{I}}=\left\{\left(x, \mathbf{v}, g B^{\theta}\right) \in \tilde{\mathcal{X}}_{\mathbf{m}^{\prime}}^{\dagger} \mid\left(x, \mathbf{v}, g B^{\theta}\right) \mapsto \mathbf{I}\right\}
$$

Let $\mathcal{I}^{\bullet}\left(\mathbf{m}^{\prime}\right)$ be as in 3.3. Then as in Lemma 2.4 (see also 3.6), we have

$$
\tilde{\mathcal{X}}_{\mathbf{m}^{\prime}}^{\dagger}=\coprod_{\mathbf{I} \in \mathcal{I} \bullet\left(\mathbf{m}^{\prime}\right)} \widetilde{\mathcal{X}}_{\mathbf{I}}
$$

where $\widetilde{\mathcal{X}}_{\mathbf{I}}$ is an irreducible component of $\widetilde{\mathcal{X}}_{\mathbf{m}^{\prime}}^{\dagger}$. Let $\pi_{\mathbf{m}^{\prime}}^{\bullet}: \widetilde{\mathcal{X}}_{\mathbf{m}^{\prime}}^{\bullet} \rightarrow \mathcal{X}_{\mathbf{m}^{\prime}}^{0}$ be the restriciton of $\pi^{(\mathbf{m})}$ on $\tilde{\mathcal{X}}_{\mathbf{m}^{\prime}}^{\dagger}$. The following result is an analogue of Proposition 2.8 (see also Proposition 3.7 ) and can be proved in a similar way. 
Proposition 4.7. Assume that $\mathbf{m}^{\prime} \leq \mathbf{m}$. Then $\left(\pi_{\mathbf{m}^{\prime}}^{\bullet}\right) ! \alpha_{0}^{*} \mathcal{E}$ is decomposed as

$$
\left.\left(\pi_{\mathbf{m}^{\prime}}^{\bullet}\right) ! \alpha_{0}^{*} \mathcal{E}\right|_{\tilde{\mathcal{X}}_{\mathbf{m}^{\prime}}^{\dagger}} \simeq \bigoplus_{\rho \in\left(\mathcal{W}_{\mathbf{m}^{\prime}, \mathcal{E}}^{\bullet} \mathcal{E}^{\wedge}\right.}\left(\operatorname{Ind}_{\mathcal{W}_{\mathbf{m}^{\prime}, \mathcal{E}}^{\mathcal{W}_{\dot{\varepsilon}}^{*}}}^{\mathcal{W}^{\bullet}} \rho\right) \otimes \operatorname{IC}\left(\mathcal{X}_{\mathbf{m}^{\prime}}^{0}, \mathcal{L}_{\rho}\right)
$$

4.8. By making use of Proposition 4.3 and Proposition 4.7, the theorem is proved by a similar argument as in 2.8 and 2.10 .

\section{UNIPOTENT VARIETY OF ENHANCED TYPE}

In this section, we study the "unipotent part" of the enhanced space, whih we call the unipotent variety of enhanced type. First we prepare some combinatorial notation.

5.1. A composition is a sequence of integers $\lambda=\left(\lambda_{1}, \lambda_{2}, \ldots\right)$ with finitely many nonzero terms. A composition $\lambda$ satisfying the property that $\lambda_{1} \geq \lambda_{2} \geq \cdots$ is called a partition. For a composition $\lambda$, we denote by $|\lambda|=\sum_{i} \lambda_{i}$ the size of $\lambda$. For a positive integer $r$, an $r$-tuple of partitions $\boldsymbol{\lambda}=\left(\lambda^{(1)}, \ldots, \lambda^{(r)}\right)$ is called an $r$-partition. We denote by $|\boldsymbol{\lambda}|=\sum_{i}\left|\lambda^{(i)}\right|$ the size of $\boldsymbol{\lambda}$. We express an $r$-partition by $\boldsymbol{\lambda}=\left(\lambda_{j}^{(i)}\right)$ with partitions $\lambda^{(i)}=\left(\lambda_{1}^{(i)}, \ldots, \lambda_{m}^{(i)}\right)$ by choosing sufficiently large $m$ so that $\lambda_{j}^{(i)}=0$ for $j>m$ and for any $i$. The set of $r$-partitions of size $n$ is denoted by $\mathcal{P}_{n, r}$. In the case where $r=1$, the set $\mathcal{P}_{n, 1}$ of partitions of $n$ is simply denoted by $\mathcal{P}_{n}$. For a given $\mathbf{m} \in \mathcal{Q}_{n, r}$, we denote by $\mathcal{P}(\mathbf{m})$ the set of $\boldsymbol{\lambda} \in \mathcal{P}_{n, r}$ such that $\left|\lambda^{(i)}\right|=m_{i}$ for each $i$.

Let $\boldsymbol{\lambda}=\left(\lambda_{j}^{(i)}\right)$ be an $r$-partition of $n$. We define a composition $c(\boldsymbol{\lambda})$ of $n$ associated to $\boldsymbol{\lambda}$ by

$$
c(\boldsymbol{\lambda})=\left(\lambda_{1}^{(1)}, \lambda_{1}^{(2)}, \ldots, \lambda_{1}^{(r)}, \lambda_{2}^{(1)}, \lambda_{2}^{(2)}, \ldots, \lambda_{2}^{(r)}, \ldots, \lambda_{m}^{(1)}, \lambda_{m}^{(2)}, \ldots, \lambda_{m}^{(r)}\right)
$$

For example, if $\boldsymbol{\lambda}=(320 ; 211 ; 411) \in \mathcal{P}_{15,3}$, we have $c(\boldsymbol{\lambda})=(324211011)$.

For a composition $\lambda=\left(\lambda_{1}, \lambda_{2}, \ldots,\right), \mu=\left(\mu_{1}, \mu_{2}, \ldots,\right)$, we denote by $\lambda \leq \mu$ if

$$
\lambda_{1}+\cdots+\lambda_{k} \leq \mu_{1}+\cdots+\mu_{k}
$$

for $k=1,2, \cdots$. We define a dominance order $\leq$ on $\mathcal{P}_{n, r}$ by the condition that $\boldsymbol{\lambda} \leq \boldsymbol{\mu}$ if $c(\boldsymbol{\lambda}) \leq c(\boldsymbol{\mu})$. In the case where $r=1$, this is the standard dominance order on the set $\mathcal{P}_{n}$.

For a partition $\lambda=\left(\lambda_{1}, \lambda_{2}, \ldots\right)$, we put $n(\lambda)=\sum_{i \geq 1}(i-1) \lambda_{i}$. We define a function $n: \mathcal{P}_{n, r} \rightarrow \mathbf{Z}$ by $n(\boldsymbol{\lambda})=\sum_{i=1}^{r} n\left(\lambda^{(i)}\right)$.

5.2. We consider the enhanced space of higher level introduced in 1.2. However in this section, we redefine them directly, without using the symmetric space setting in 1.2. For an integer $r \geq 1$, we consider a variety $\mathcal{X}_{\text {uni }}=G_{\text {uni }} \times V^{r-1}$, where $V$ is an $n$-dimensional vector space over $\mathbf{k}, G=G L(V)$ and $G_{\text {uni }}$ is the unipotent variety of $G$. We fix a basis $\left\{e_{1}, \ldots, e_{n}\right\}$ of $V$, and define $M_{i}$ as a subspace of $V$ 
spanned by $e_{1}, \ldots, e_{i}$. Let $B$ be a Borel subgroup of $G$ which is the stabilizer of the total flag $\left(M_{i}\right)$. Let $T$ be a maximal torus of $B$ such that $\left\{e_{i}\right\}$ are weight vectors for $T$. Then $B=T U$, where $U$ is the unipotent radical of $B$. For $\mathbf{m} \in \mathcal{Q}_{n, r}$, let $\mathbf{p}(\mathbf{m})=\left(p_{1}, \ldots, p_{r}\right)$ be as in 1.2. We define

$$
\begin{aligned}
& \widetilde{\mathcal{X}}_{\mathbf{m}, \text { uni }}=\left\{(x, \mathbf{v}, g B) \in G_{\text {uni }} \times V^{r-1} \times G / B \mid g^{-1} x g \in U, g^{-1} \mathbf{v} \in \prod_{i=1}^{r-1} M_{p_{i}}\right\} \\
& \mathcal{X}_{\mathbf{m}, \text { uni }}=\bigcup_{g \in G} g\left(U \times \prod_{i=1}^{r-1} M_{p_{i}}\right) .
\end{aligned}
$$

We define a map $\pi_{1}^{(\mathbf{m})}: \widetilde{\mathcal{X}}_{\mathbf{m}, \text { uni }} \rightarrow \mathcal{X}_{\text {uni }}$ by $(x, \mathbf{v}, g B) \mapsto(x, \mathbf{v})$. Clearly $\mathcal{X}_{\mathbf{m}, \text { uni }}=$ $\operatorname{Im} \pi_{1}^{(\mathbf{m})}$, and $\mathcal{X}_{\mathbf{m} \text {,uni }}$ coincides with $\mathcal{X}_{\text {uni }}$ in the case where $\mathbf{m}=(n, 0, \ldots, 0)$. Since $\widetilde{\mathcal{X}}_{\mathbf{m} \text {,uni }} \simeq G \times \times^{B}\left(U \times \prod_{i} M_{p_{i}}\right), \widetilde{\mathcal{X}}_{\mathbf{m} \text {,uni }}$ is smooth and irreducible. Since $\pi_{1}^{(\mathbf{m})}$ is proper, $\mathcal{X}_{\mathrm{m}, \text { uni }}$ is a closed irreducible subvariety of $\mathcal{X}_{\text {uni }}$.

5.3. We shall define a partition of $\mathcal{X}_{\text {uni }}$ into pieces $X_{\boldsymbol{\lambda}}$ indexed by $\boldsymbol{\lambda} \in \mathcal{P}_{n, r}$,

$$
\mathcal{X}_{\text {uni }}=\coprod_{\lambda \in \mathcal{P}_{n, r}} X_{\boldsymbol{\lambda}}
$$

satisfying the property such that $X_{\boldsymbol{\lambda}}$ is $G$-stable, and that if $(x, \mathbf{v}) \in X_{\boldsymbol{\lambda}}$, then the Jordan type of $x$ is $\lambda^{(1)}+\cdots+\lambda^{(r)}$. It is known by [AH] and [T] that the set of $G$-orbits in $G_{\text {uni }} \times V$ is finite, and they are parametrized by $\mathcal{P}_{n, 2}$. The labelling is given as follows; take $(x, v) \in G_{\text {uni }} \times V$. Let $E^{x}=\{y \in \operatorname{End}(V) \mid x y=y x\}$. $E^{x}$ is a subalgebra of $\operatorname{End}(V)$ stable by the multiplication of $x$. If we put $W=E^{x} v$, $W$ is an $x$-stable subspace of $V$. We denote by $\lambda^{(1)}$ the Jordan type of $\left.x\right|_{W}$, and by $\lambda^{(2)}$ the Jordan type of $\left.x\right|_{V / W}$. Then the Jordan type of $x$ is $\lambda^{(1)}+\lambda^{(2)}$ and $\boldsymbol{\lambda}=\left(\lambda^{(1)}, \lambda^{(2)}\right) \in \mathcal{P}_{n, 2}$. We denote by $\mathcal{O}_{\boldsymbol{\lambda}}$ the $G$-orbit containing $(x, v)$. This gives the required labelling of $G$-orbits in $G_{\text {uni }} \times V$.

In general, we define $X_{\boldsymbol{\lambda}}$ by induction on $r$. Take $(x, \mathbf{v}) \in \mathcal{X}_{\text {uni }}$ with $\mathbf{v}=$ $\left(v_{1}, \ldots, v_{r-1}\right)$. Put $W=E_{G}^{x} v_{1}, \bar{V}=V / W$ and $\bar{G}=G L(\bar{V})$. We consider the variety $\mathcal{X}_{\text {uni }}^{\prime}=\bar{G} \times \bar{V}^{r-2}$. Assume that $\left(x, v_{1}\right) \in G_{\text {uni }} \times V$ is of type $\left(\lambda^{(1)}, \nu^{\prime}\right)$, where $\nu=\lambda^{(1)}+\nu^{\prime}$ is the type of $x$. Let $\bar{x}$ be the restriction of $x$ on $\bar{V}$. Then the type of $\bar{x} \in G L(\bar{V})$ is $\nu^{\prime}$. Put $\overline{\mathbf{v}}=\left(\bar{v}_{2}, \ldots \bar{v}_{r-1}\right)$, where $\bar{v}_{i}$ is the image of $v_{i}$ on $\bar{V}$. Thus $(\bar{x}, \overline{\mathbf{v}}) \in \mathcal{X}_{\text {uni }}^{\prime}$. By induction, we have a partition $\mathcal{X}_{\text {uni }}^{\prime}=\coprod_{\boldsymbol{\mu} \in \mathcal{P}_{n^{\prime}, r-1}} X_{\boldsymbol{\mu}}^{\prime}$, where $\operatorname{dim} \bar{V}=n^{\prime}$. Thus there exists a unique $X_{\boldsymbol{\lambda}^{\prime}}^{\prime}$ containing $(\bar{x}, \overline{\mathbf{v}})$. If we write $\boldsymbol{\lambda}^{\prime}=\left(\lambda^{(2)}, \ldots, \lambda^{(r)}\right)$, we have $\lambda^{(2)}+\cdots+\lambda^{(r)}=\nu^{\prime}$. It follows that $\boldsymbol{\lambda}=\left(\lambda^{(1)}, \ldots \lambda^{(r)}\right) \in$ $\mathcal{P}_{n, r}$. We define the type of $(x, \mathbf{v})$ by $\boldsymbol{\lambda}$, and define a subset $X_{\boldsymbol{\lambda}}$ of $\mathcal{X}_{\text {uni }}$ as the set of all $(x, \mathbf{v})$ with type $\boldsymbol{\lambda}$. Then $X_{\boldsymbol{\lambda}}$ is a $G$-stable subset of $\mathcal{X}_{\text {uni }}$, and we obtain the required partition (5.3.1).

We show the following proposition. 
Proposition 5.4. Let $\boldsymbol{\lambda}=\left(\lambda^{(1)}, \ldots, \lambda^{(r)}\right) \in \mathcal{P}_{n, r}$. Then $X_{\boldsymbol{\lambda}}$ is a smooth irreducible variety with

$$
\operatorname{dim} X_{\boldsymbol{\lambda}}=\left(n^{2}-n-2 n(\boldsymbol{\lambda})\right)+\sum_{i=1}^{r-1}(r-i)\left|\lambda^{(i)}\right| .
$$

Proof. We may assume that $r \geq 2$. Put $\nu=\lambda^{(1)}+\cdots+\lambda^{(r)}$ and $\nu^{\prime}=\lambda^{(2)}+\cdots+\lambda^{(r)}$. Let $\mathcal{O}=\mathcal{O}_{\left(\lambda^{(1)}, \nu^{\prime}\right)}$ be the $G$-orbit in $G_{\text {uni }} \times V$ corresponding to $\left(\lambda^{(1)}, \nu^{\prime}\right) \in \mathcal{P}_{n, 2}$, and $\mathcal{O}_{\nu}$ be the $G$-orbit in $G_{\text {uni }}$ corresponding to $\nu \in \mathcal{P}_{n}$. . We have surjective maps $f_{\boldsymbol{\lambda}}: X_{\boldsymbol{\lambda}} \rightarrow \mathcal{O},(x, \mathbf{v}) \mapsto\left(x, v_{1}\right)$, and $h_{\boldsymbol{\lambda}}: X_{\boldsymbol{\lambda}} \rightarrow \mathcal{O}_{\nu},(x, \mathbf{v}) \mapsto x$. For each $x \in \mathcal{O}_{\nu}$, put $h_{\boldsymbol{\lambda}}^{-1}(x)=X_{\boldsymbol{\lambda}, x}$. The proposition certainly holds if $r=2$ by [AH, Proposition 2.8]. We show the proposition, together with the statement (5.4.2), by induction on $r$.

(5.4.2) $X_{\boldsymbol{\lambda}, x}$ is a smooth and irreducible variety with

$$
\operatorname{dim} X_{\boldsymbol{\lambda}, x}=\sum_{i=1}^{r-1}(r-i)\left|\lambda^{(i)}\right| .
$$

We assume that (5.4.2) holds for $\mathcal{X}_{\text {uni }}$ with smaller $r$. For a fixed $\left(x, v_{1}\right) \in \mathcal{O}$, we consider the variety $\mathcal{X}_{\text {uni }}^{\prime}$ as in 5.3. Let $\boldsymbol{\lambda}^{\prime}=\left(\lambda^{(2)}, \ldots, \lambda^{(r)}\right)$. Then by the discussion in 5.3, we see that $f_{\lambda}^{-1}\left(x, v_{1}\right) \simeq X_{\lambda^{\prime}, \bar{x}}^{\prime} \times W^{r-2}$. $Z_{G}\left(x, v_{1}\right)$ stabilizes the subspace $W$ and so acts on $X_{\boldsymbol{\lambda}^{\prime}, \bar{x}}^{\prime}$. We have

$$
X_{\boldsymbol{\lambda}} \simeq G \times \times^{Z_{G}\left(x, v_{1}\right)}\left(X_{\boldsymbol{\lambda}^{\prime}, \bar{x}}^{\prime} \times W^{r-2}\right) .
$$

By induction, $X_{\boldsymbol{\lambda}^{\prime}, \bar{x}}^{\prime}$ is smooth and irreducible. Hence $X_{\boldsymbol{\lambda}}$ is smooth and irreducible. Since

$$
X_{\boldsymbol{\lambda}, x} \simeq Z_{G}(x) \times{ }^{Z_{G}\left(x, v_{1}\right)}\left(X_{\boldsymbol{\lambda}^{\prime}, \bar{x}}^{\prime} \times W^{r-2}\right),
$$

and $Z_{G}(x)$ is connected, $X_{\boldsymbol{\lambda}, x}$ is also smooth and irreducible. This proves the first statement of (5.4.1) and (5.4.2). We shall compute $\operatorname{dim} X_{\boldsymbol{\lambda}}$ and $\operatorname{dim} X_{\boldsymbol{\lambda}, x}$. By (5.4.3), we have

$$
\operatorname{dim} X_{\boldsymbol{\lambda}}=\operatorname{dim} \mathcal{O}_{\left(\lambda^{(1)}, \nu^{\prime}\right)}+\operatorname{dim} X_{\boldsymbol{\lambda}^{\prime}, \bar{x}}^{\prime}+(r-2)\left|\lambda^{(1)}\right| .
$$

Here $\operatorname{dim} \mathcal{O}_{\left(\lambda^{(1)}, \nu^{\prime}\right)}=n^{2}-n-2 n(\nu)+\left|\lambda^{(1)}\right|$ by [AH, Prop. 2.8]. By applying (5.4.2) to $X_{\lambda^{\prime}, \bar{x}}^{\prime}$, we have

$$
\operatorname{dim} X_{\lambda^{\prime}, \bar{x}}^{\prime}=\sum_{i=2}^{r-1}(r-i)\left|\lambda^{(i)}\right| .
$$

By substituting those formulas to (5.4.5), we obtain (5.4.1) (note that $n(\nu)=n(\boldsymbol{\lambda})$ ). 
By comparing (5.4.3) and (5.4.4), we have

$$
\begin{aligned}
\operatorname{dim} X_{\boldsymbol{\lambda}, x} & =\operatorname{dim} X_{\boldsymbol{\lambda}}-\left(\operatorname{dim} G-\operatorname{dim} Z_{G}(x)\right) \\
& =\operatorname{dim} X_{\boldsymbol{\lambda}}-\operatorname{dim} \mathcal{O}_{\nu} .
\end{aligned}
$$

Since $\operatorname{dim} \mathcal{O}_{\nu}=n^{2}-n-2 n(\nu)$, (5.4.2) follows from (5.4.1). This proves the proposition.

5.5. Let $\boldsymbol{\lambda}=\left(\lambda^{(1)}, \ldots, \lambda^{(r)}\right) \in \mathcal{P}(\mathbf{m})$. We write the dual partition $\left(\lambda^{(i)}\right)^{*}$ of $\lambda^{(i)}$ as $\left(\mu_{1}^{(i)} \leq \mu_{2}^{(i)} \leq \cdots \leq \mu_{\ell_{i}}^{(i)}\right)$, in the increasing order, where $\ell_{i}=\lambda_{1}^{(i)}$. For each $1 \leq i \leq r, 1 \leq j<\ell_{i}$, we define an integer $n(i, j)$ by

$$
n(i, j)=\left(\left|\lambda^{(1)}\right|+\cdots+\left|\lambda^{(i-1)}\right|\right)+\mu_{1}^{(i)}+\cdots+\mu_{j}^{(i)} .
$$

Let $P=P_{\boldsymbol{\lambda}}$ be the stabilizer of a partial flag $\left(M_{n(i, j)}\right)$ in $G$, and $U_{P}$ the unipotent radical of $P$. Let us define a variety $\widetilde{X}_{\boldsymbol{\lambda}}$ by

$$
\widetilde{X}_{\boldsymbol{\lambda}}=\left\{(x, \mathbf{v}, g P) \in G_{\text {uni }} \times V^{r-1} \times G / P \mid g^{-1} x g \in U_{P}, g^{-1} \mathbf{v} \in \prod_{i=1}^{r-1} M_{p_{i}}\right\}
$$

We define a map $\pi_{\boldsymbol{\lambda}}: \widetilde{X}_{\boldsymbol{\lambda}} \rightarrow \mathcal{X}_{\text {uni }}$ by $(x, \mathbf{v}, g P) \mapsto(x, \mathbf{v})$. Then $\pi_{\boldsymbol{\lambda}}$ is a proper map. Since $\tilde{X}_{\boldsymbol{\lambda}} \simeq G \times^{P}\left(U_{P} \times \prod_{i} M_{p_{i}}\right), \widetilde{X}_{\boldsymbol{\lambda}}$ is smooth and irreducible.

We have the following lemma.

Lemma 5.6. Let $\boldsymbol{\lambda}=\left(\lambda^{(1)}, \ldots, \lambda^{(r)}\right) \in \mathcal{P}_{n, r}$.

(i) $\operatorname{dim} \tilde{X}_{\boldsymbol{\lambda}}=\operatorname{dim} X_{\boldsymbol{\lambda}}$.

(ii) $\operatorname{Im} \pi_{\boldsymbol{\lambda}}=\bar{X}_{\boldsymbol{\lambda}}$, where $\bar{X}_{\boldsymbol{\lambda}}$ is the closure of $X_{\boldsymbol{\lambda}}$ in $\mathcal{X}_{\mathrm{uni}}$.

Proof. We have

$$
\begin{aligned}
\operatorname{dim} \widetilde{X}_{\boldsymbol{\lambda}} & =\operatorname{dim} G / P+\operatorname{dim} U_{P}+\operatorname{dim} \prod_{i=1}^{r-1} M_{p_{i}} \\
& =2 \operatorname{dim} U_{P}+\sum_{i=1}^{r-1}(r-i)\left|\lambda^{(i)}\right| .
\end{aligned}
$$

Here $\operatorname{dim} U_{P}=(\operatorname{dim} G-\operatorname{dim} L) / 2$, where $L$ is a Levi subgroup of $P$, and

$$
\operatorname{dim} L=\sum_{i, j}\left(\mu_{j}^{(i)}\right)^{2}=n+2 n(\boldsymbol{\lambda})
$$

The second equality follows form the formula $n\left(\lambda^{*}\right)=\sum_{i} \lambda_{i}\left(\lambda_{i}-1\right) / 2$ for a partition $\lambda=\left(\lambda_{1}, \lambda_{2}, \ldots\right)$ and its dual $\lambda^{*}$. By comparing this with Proposition 5.4, we obtain (i). 
We show (ii). Take $(x, \mathbf{v}) \in X_{\boldsymbol{\lambda}}$. By the construction of $X_{\boldsymbol{\lambda}}$ in 5.3, one can find a sequence of $x$-stable subspaces $V_{p_{1}} \subset V_{p_{2}} \subset \cdots \subset V_{p_{r}}=V$ of $V$ such that $v_{i} \in V_{p_{i}}$ and that the restriction of $x$ on $V_{p_{i}} / V_{p_{i-1}}$ has type $\lambda^{(i)}$. It is well-known that there exists an $x$-stable flag $\left(V_{n(i, j)}\right)$ which is a refinement of $\left(V_{p_{i}}\right)$ such that $V_{n(i, j)}=g M_{n(i, j)}$ for some $g \in G$ and that $g^{-1} x g \in U_{P}$ (see [AH, Prop. $\left.3.3(4)\right]$ ). It follows that $(x, \mathbf{v}, g P) \in \widetilde{X}_{\boldsymbol{\lambda}}$, and we see that $(x, \mathbf{v}) \in \operatorname{Im} \pi_{\boldsymbol{\lambda}}$. This proves that $X_{\boldsymbol{\lambda}} \subset \operatorname{Im} \pi_{\boldsymbol{\lambda}}$. Since $\pi_{\boldsymbol{\lambda}}$ is proper, $\operatorname{Im} \pi_{\boldsymbol{\lambda}}$ is a closed subset of $\mathcal{X}_{\text {uni }}$ and we have $\bar{X}_{\boldsymbol{\lambda}} \subset \operatorname{Im} \pi_{\boldsymbol{\lambda}}$. Since $\bar{X}_{\boldsymbol{\lambda}}$ and $\operatorname{Im} \pi_{\boldsymbol{\lambda}}$ are both irreducible, (i) implies that $\operatorname{Im} \pi_{\boldsymbol{\lambda}}=\bar{X}_{\boldsymbol{\lambda}}$. Hence (ii) holds. The lemma is proved.

Remark 5.7. In the case where $r=2$, Achar-Henderson proved in [AH, Prop. 3.3] that the map $\pi_{\boldsymbol{\lambda}}: \widetilde{X}_{\boldsymbol{\lambda}} \rightarrow \bar{X}_{\boldsymbol{\lambda}}$ is a resolution fo singularities for $\bar{X}_{\boldsymbol{\lambda}}$. By a similar argument, by usnig Lemma 5.6 (ii), one can prove that $\pi_{\boldsymbol{\lambda}}$ gives a resolution of singularities for $\bar{X}_{\boldsymbol{\lambda}}$ for any $r \geq 2$.

5.8. Recall the map $\pi_{1}^{(m)}: \widetilde{\mathcal{X}}_{\mathbf{m} \text {,uni }} \rightarrow \mathcal{X}_{\mathbf{m} \text {,uni }}$ in 5.2 for $\mathbf{m}=\left(m_{1}, \ldots, m_{r}\right) \in \mathcal{Q}_{n, r}$. Let us define $\boldsymbol{\lambda}(\mathbf{m})=\left(\lambda^{(1)}, \ldots, \lambda^{(r)}\right)$ by the condition that $\lambda^{(i)}=\left(m_{i}\right)$ for each $i$. We consider the varieties $\widetilde{X}_{\boldsymbol{\lambda}}$ in 5.5 for $\boldsymbol{\lambda}=\boldsymbol{\lambda}(\mathbf{m})$. In this case, $P=B$. $\tilde{X}_{\boldsymbol{\lambda}}$ is isomorphic to $\widetilde{\mathcal{X}}_{\mathbf{m} \text {,uni }}$, and $\pi_{\boldsymbol{\lambda}}$ is identified with $\pi_{1}^{(\mathbf{m})}$. We have the following result.

Proposition 5.9. For $\mathbf{m} \in \mathcal{Q}_{n, r}$, we have

(i) $\mathcal{X}_{\mathbf{m}, \text { uni }}=\bar{X}_{\boldsymbol{\lambda}(\mathbf{m})}$.

(ii) $\operatorname{dim} \mathcal{X}_{\mathbf{m}, \text { uni }}=n^{2}-n+\sum_{i=1}^{r-1}(r-i) m_{i}$.

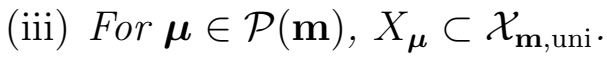

Proof. (i) is a direct consequence of Lemma 5.6 (ii) in view of 5.8. (ii) follows from (i) and Proposition 5.4. We show (iii). Take $(x, \mathbf{v}) \in X_{\boldsymbol{\mu}}$. As in the proof of Lemma 5.6, there exists an $x$-stable partial flag $\left(V_{n(i, j)}\right)$ with respect to $\boldsymbol{\mu}$. By our assumption, $v_{i} \in V_{p_{i}}$ for each $i$. Then one can find an $x$-stable total flag $\left(V_{i}\right)$ as a refinement of this $\left(V_{n(i, j)}\right)$. This shows that $(x, \mathbf{v})$ is contained in $\operatorname{Im} \pi_{1}^{(\mathbf{m})}=\mathcal{X}_{\mathbf{m} \text {,uni. }}$.

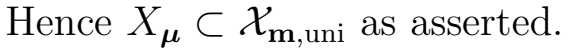

5.10. In the case where $r=2$, there exists a normal basis for $(x, v) \in G_{\text {uni }} \times V$ (cf. [AH, 2.2], [T]). If $r \geq 3$, one can not expect such a basis since $\mathcal{X}_{\text {uni }}$ has infinitely many $G$-orbits. However, one can find typical elements in $X_{\boldsymbol{\lambda}}$ as follows; put $\nu=$ $\left(\nu_{1}, \ldots, \nu_{\ell}\right) \in \mathcal{P}_{n}$ for $\nu=\lambda^{(1)}+\cdots+\lambda^{(r)}$. Take $x \in G_{\text {uni }}$ of Jordan type $\nu$, and let $\left\{u_{j, k} \mid 1 \leq j \leq \ell, 1 \leq k \leq \nu_{j}\right\}$ be a Jordan basis of $x$ in $V$ having the property $(x-1) u_{j, k}=u_{j, k-1}$ with the convention that $u_{j, 0}=0$. We define $v_{i} \in V$ for $i=1, \ldots, r-1$ by the condition that

$$
v_{i}=\sum_{1 \leq j \leq \ell} u_{j, \lambda_{j}^{(1)}+\cdots+\lambda_{j}^{(i)}}
$$

and put $\mathbf{v}=\left(v_{1}, \ldots, v_{r-1}\right)$. Let $W_{i}$ be a subspace of $V$ spanned by the basis

$$
\left\{u_{j, k} \mid 1 \leq j \leq \ell, 1 \leq k \leq \lambda_{j}^{(1)}+\cdots+\lambda_{j}^{(i)}\right\} .
$$


Then $W_{1} \subset W_{2} \subset \cdots \subset W_{r-1} \subset V$, and $W_{i}$ is an $x$-stable subspace of $V$ such that $v_{i} \in W_{i}$. Let $\bar{x}$ be the restriction of $x$ on $\bar{V}=V / W_{i-1}$, and $\bar{v}_{i}$ be the image of $v_{i}$ on $\bar{V}$. Put $\bar{G}=G L(\bar{V})$. Then $E^{\bar{x}} \bar{v}_{i}$ coincides with $W_{i} / W_{i-1}$, and the restriciton of $x$ on $W_{i} / W_{i-1}$ has type $\lambda^{(i)}$. It follows that $(x, \mathbf{v}) \in X_{\boldsymbol{\lambda}}$. Such an element $(x, \mathbf{v}) \in X_{\boldsymbol{\lambda}}$ is called a standard element. More generally, we consider an element $\mathbf{w}=\left(w_{1}, \ldots, w_{r-1}\right)$ of the form

$$
w_{i}=\sum_{j=1}^{\ell} \sum_{k=1}^{\lambda_{j}^{(1)}+\cdots+\lambda_{j}^{(i)}} a_{j, k} u_{j, k}
$$

with $a_{j, k} \in \mathbf{k}$ such that $a_{j, \lambda_{j}^{(1)}+\cdots+\lambda_{j}^{(i)}} \neq 0$. Then $w_{i} \in W_{i}$, and $E^{\bar{x}} \bar{w}_{i}$ coincides with $W_{i} / W_{i-1}$. Hence $(x, \mathbf{w}) \in X_{\boldsymbol{\lambda}}$. Here $\left(x, w_{i}\right)$ is conjugate to an element $\left(x, v_{i}\right)$ as above under the group $Z_{G}(x)$ for each $i$. We call $(x, \mathbf{w})$ a semi-standard element. We define a set $X_{\boldsymbol{\lambda}}^{0}$ as the set of all $G$-conjugates of $(x, \mathbf{w})$. Hence $X_{\boldsymbol{\lambda}}^{0}$ is a $G$-stable subset of $X_{\boldsymbol{\lambda}}$. We note that

$X_{\lambda}^{0}$ is a $G$-stable open dense subset of $X_{\lambda}$.

In fact, take $(x, \mathbf{v}) \in X_{\lambda}^{0}$. Under the notation in the proof of Proposition 5.4, put $X_{\boldsymbol{\lambda}, x}^{0}=X_{\boldsymbol{\lambda}}^{0} \cap X_{\boldsymbol{\lambda}, x}$. Then under the isomorphism in (5.4.4), we have

$$
X_{\boldsymbol{\lambda}, x}^{0} \simeq Z_{G}(x) \times^{Z_{G}\left(x, v_{1}\right)}\left(X_{\boldsymbol{\lambda}^{\prime}, \bar{x}}^{\prime 0} \times\left(W^{0}\right)^{r-2}\right),
$$

where $X_{\boldsymbol{\lambda}^{\prime}, \bar{x}}^{\prime 0}$ is a similar variety as $X_{\boldsymbol{\lambda}, x}^{0}$ defined for $X_{\boldsymbol{\lambda}^{\prime}}^{\prime}$, and $W^{0}$ is an open dense subset of $W$. Hence by induction on $r, X_{\boldsymbol{\lambda}, x}^{0}$ is an open dense subset of $X_{\boldsymbol{\lambda}}$. Since, under the isomorphism in $(5.4 .3), X_{\lambda}^{0}$ can be written as

$$
X_{\boldsymbol{\lambda}}^{0} \simeq G \times^{Z_{G}\left(x, v_{1}\right)}\left(X_{\boldsymbol{\lambda}^{\prime}, \bar{x}}^{\prime 0} \times\left(W^{0}\right)^{r-2}\right),
$$

$X_{\boldsymbol{\lambda}}^{0}$ is open dense in $X_{\boldsymbol{\lambda}}$. Hence (5.10.3) holds.

Concerning the closure $\bar{X}_{\boldsymbol{\lambda}}$, we have the following result.

Proposition 5.11. For each $\boldsymbol{\lambda} \in \mathcal{P}_{n, r}$, we have

$$
\bar{X}_{\lambda} \subset \bigcup_{\mu \leq \lambda} X_{\mu}
$$

For the proof of the proposition, we need a lemma.

Lemma 5.12. Assume that $(x, \mathbf{v}) \in X_{\boldsymbol{\lambda}}$ is a semi-standard element with $\mathbf{v}=$ $\left(v_{1}, \ldots, v_{r-1}\right)$. Let $U_{i}$ be the $\mathbf{k}[x]$-submodule of $V$ generated by $v_{i}$. Then $\operatorname{dim} U_{i}=$ $\lambda_{1}^{(1)}+\cdots+\lambda_{1}^{(i)}$. The Jordan type of the restriction of $x$ on $V / U_{i}$ is $\xi=\left(\xi_{1}, \ldots, \xi_{\ell}\right)$, where

$$
\xi_{j}=\lambda_{j}^{(i+1)}+\cdots+\lambda_{j}^{(r)}+\lambda_{j+1}^{(1)}+\cdots+\lambda_{j+1}^{(i)}, \quad \text { for } j=1, \ldots, \ell
$$


Proof. In the case where $r=2$, this result was proved in Lemma 2.5 in [AH]. The general case is reduced to the case where $r=2$, by considering the double partition $\left(\mu ; \mu^{\prime}\right)$ with $\mu=\lambda^{(1)}+\cdots+\lambda^{(i)}, \mu^{\prime}=\lambda^{(i+1)}+\cdots+\lambda^{(r)}$.

5.13. We prove the proposition following the strategy in the proof of (a part of) $\left[\right.$ AH, Th. 3.9]. We show that $\boldsymbol{\mu} \leq \boldsymbol{\lambda}$ if $\left(x^{\prime}, \mathbf{v}^{\prime}\right) \in \bar{X}_{\boldsymbol{\lambda}}$ is of type $\boldsymbol{\mu}$. For this, it is enough to show that

$$
\begin{aligned}
\sum_{i=1}^{k}\left(\mu_{i}^{(1)}+\right. & \left.\cdots+\mu_{i}^{(r)}\right)+\left(\mu_{k+1}^{(1)}+\cdots \mu_{k+1}^{(a)}\right) \\
& \leq \sum_{i=1}^{k}\left(\lambda_{i}^{(1)}+\cdots+\lambda_{i}^{(r)}\right)+\left(\lambda_{k+1}^{(1)}+\cdots+\lambda_{k+1}^{(a)}\right)
\end{aligned}
$$

for $k=0, \ldots, \ell$ and $a=0, \ldots, r-1$ (we put $\lambda_{j}^{(0)}=\mu_{j}^{(0)}=0$ by convention). Put $\nu=\lambda^{(1)}+\cdots+\lambda^{(r)}$ and $\nu^{\prime}=\mu^{(1)}+\cdots+\mu^{(r)}$. Then the Jordan type of $x^{\prime}$ is $\nu^{\prime}$. Let $\mathcal{O}_{\nu}$ be the $G$-orbit in $G_{\text {uni }}$ corresponding to $\nu \in \mathcal{P}_{n}$. Since $X_{\boldsymbol{\lambda}} \subset \mathcal{O}_{\nu} \times V^{r-1}$, we have $\bar{X}_{\boldsymbol{\lambda}} \subseteq \overline{\mathcal{O}}_{\nu} \times V^{r-1}$. Since $\left(x^{\prime}, \mathbf{v}^{\prime}\right) \in \bar{X}_{\boldsymbol{\lambda}}$, we have $x^{\prime} \in \overline{\mathcal{O}}_{\nu}$ and so $\nu^{\prime} \leq \nu$. This proves (5.13.1) in the case where $a=0$. By a similar argument as in the proof of [AH, Th. 3.9], it follows from Lemma 5.12 that

$$
\sum_{i=1}^{k}\left(\lambda_{i}^{(1)}+\cdots+\lambda_{i}^{(r)}\right)+\left(\lambda_{k+1}^{(1)}+\cdots+\lambda_{k+1}^{(a)}\right)
$$

is the maximal possible dimension of the $x$-stable subspace $\mathbf{k}[x]\left\langle v_{a}, u_{1}, \ldots, u_{k}\right\rangle$ of $V$, a $\mathbf{k}[x]$-module generated by $v_{a}, u_{1}, \ldots, u_{k}$, where $(x, \mathbf{v})$ is a semi-standard element in $X_{\boldsymbol{\lambda}}$ with $\mathbf{v}=\left(v_{1}, \ldots, v_{r-1}\right)$, and $u_{1}, \ldots, u_{k}$ runs over the elements in $V$. We note that the condition

$$
\operatorname{dim} \mathbf{k}[x]\left\langle v_{a}, u_{1}, \ldots, u_{k}\right\rangle \leq N_{a}, \quad\left(u_{1}, \ldots, u_{k} \in V, 1 \leq a \leq r-1\right)
$$

for given $\left\{N_{a} \mid 1 \leq a \leq r-1\right\}$ is a closed condition on $(x, \mathbf{v}) \in \mathcal{X}_{\text {uni }}$ (i.e., the set of $(x, \mathbf{v})$ satisfying the above condition is a closed subset of $\left.\mathcal{X}_{\text {uni }}\right)$ since the condition for a fixed $a$ is a closed condition on $\mathcal{X}_{\text {uni }}$ by [loc. cit.]. Since $\bar{X}_{\boldsymbol{\lambda}}^{0}=\bar{X}_{\boldsymbol{\lambda}}$ by (5.10.3), for any element $\left(x^{\prime}, \mathbf{v}^{\prime}\right) \in \bar{X}_{\boldsymbol{\lambda}}$, the dimension of $\mathbf{k}\left[x^{\prime}\right]\left\langle v_{a}^{\prime}, u_{1}, \ldots, u_{k}\right\rangle$ is dominated by the number in (5.13.2). The assertion (5.13.1) then follows from this. Hence Proposition 5.11 holds.

Combining with the previous results, we have the following.

Proposition 5.14. $X_{\boldsymbol{\lambda}}$ is open dense in $\bar{X}_{\boldsymbol{\lambda}}$. Hence $X_{\boldsymbol{\lambda}}$ is a $G$-stable locally closed, smooth, irreducible subvariety of $\mathcal{X}_{\text {uni }}$.

Proof. Since $X_{\boldsymbol{\lambda}}=\bar{X}_{\boldsymbol{\lambda}} \backslash \bigcup_{\boldsymbol{\mu}<\boldsymbol{\lambda}} \bar{X}_{\boldsymbol{\mu}}$ by Proposition $5.11, X_{\boldsymbol{\lambda}}$ is open in $\bar{X}_{\boldsymbol{\lambda}} \cdot X_{\boldsymbol{\lambda}}$ is smooth and irreducible by Proposition 5.4. 
5.15. We give here some examples on the closure relation of $X_{\boldsymbol{\lambda}}$. First assume that $n=1$ and $r$ is arbitrary. Then $G=G L_{1} \simeq \mathbf{k}^{*}$, the multiplicative group of k. We have $G_{\text {uni }}=\{1\}$, and $\mathcal{X}_{\text {uni }} \simeq \mathbf{k}^{r-1}$. $\mathcal{P}_{1, r}$ consists of $\left\{\boldsymbol{\lambda}_{1}, \ldots, \boldsymbol{\lambda}_{r}\right\}$, where $\boldsymbol{\lambda}_{i}=\left(\lambda^{(1)}, \ldots, \lambda^{(r)}\right)$ with $\lambda^{(r+1-i)}=(1)$ and $\lambda^{(j)}=\emptyset$ for $j \neq r+1-i$ Thus the dominance order in $\mathcal{P}_{1, r}$ is given as $\boldsymbol{\lambda}_{1}<\boldsymbol{\lambda}_{2}<\cdots<\boldsymbol{\lambda}_{r}$. Under the identification $\mathcal{X}_{\text {uni }} \simeq \mathbf{k}^{r-1}$, we have

$$
\begin{aligned}
X_{\boldsymbol{\lambda}_{i}} & =\left\{\left(v_{1}, \ldots, v_{r-1}\right) \in \mathbf{k}^{r-1} \mid v_{1}=\cdots=v_{r-i}=0, v_{r-i+1} \in \mathbf{k}^{*}\right\} \\
& \simeq \begin{cases}\mathbf{k}^{i-2} \times \mathbf{k}^{*} & \text { if } i \geq 2, \\
\{0\} & \text { if } i=1 .\end{cases}
\end{aligned}
$$

Thus $X_{\boldsymbol{\lambda}_{i}}$ is a locally closed, smooth irreducible subvariety of $\mathbf{k}^{r-1}$. Since $G \simeq \mathbf{k}^{*}$, $X_{\boldsymbol{\lambda}_{1}}=\{0\}$ and $X_{\boldsymbol{\lambda}_{2}} \simeq \mathbf{k}^{*}$ are single $G$-orbits, but other $X_{\boldsymbol{\lambda}_{i}}$ are a union of infinitely many $G$-orbits. Moreover $\bar{X}_{\boldsymbol{\lambda}_{i}} \simeq \mathbf{k}^{i-1}$, and the closure relation is given as

$$
\bar{X}_{\boldsymbol{\lambda}_{i}}=\bigcup_{j \leq i} X_{\boldsymbol{\lambda}_{j}}
$$

This is an analogue of the result in the case where $r=2$, which asserts that $\bar{X}_{\boldsymbol{\lambda}}$ is a union of $X_{\boldsymbol{\mu}}$ for $\boldsymbol{\mu} \leq \boldsymbol{\lambda}$ ([AH, Th. 3.9]).

However, such a relation does not hold in general for $r \geq 3$. In fact, there exists $\boldsymbol{\mu}<\boldsymbol{\lambda}$ such that $X_{\boldsymbol{\mu}} \cap \bar{X}_{\boldsymbol{\lambda}} \neq \emptyset$ and that $X_{\boldsymbol{\mu}} \not \subset \bar{X}_{\boldsymbol{\lambda}}$ as the following example shows. We consider the case where $n=2, r=3$, hence $\operatorname{dim} V=2$. Take $\boldsymbol{\lambda}=(1 ;-; 1)$ and $\boldsymbol{\mu}=\left(1^{2} ;-;-\right)$. Then we have $\boldsymbol{\mu}<\boldsymbol{\lambda}$. It is easy to check that

$$
\begin{aligned}
& X_{\boldsymbol{\lambda}}=\left\{\left(x,\left(v_{1}, v_{2}\right)\right) \in G_{\text {uni }} \times V^{2} \mid x \neq 1, v_{1} \in \operatorname{Ker}(x-1) \backslash\{0\}, v_{2} \in \operatorname{Ker}(x-1)\right\}, \\
& X_{\boldsymbol{\mu}}=\left\{\left(x,\left(v_{1}, v_{2}\right)\right) \in G_{\text {uni }} \times V^{2} \mid x=1, v_{1} \in V \backslash\{0\}, v_{2} \in V\right\} .
\end{aligned}
$$

Then for any element $(x, \mathbf{v}) \in X_{\boldsymbol{\lambda}}$, we have $v_{2} \in \mathbf{k} v_{1}$. It follows that $v_{2} \in \mathbf{k} v_{1}$ for $(x, \mathbf{v}) \in \bar{X}_{\boldsymbol{\lambda}}$, and we have $X_{\boldsymbol{\mu}} \cap \bar{X}_{\boldsymbol{\lambda}}=\left\{\left(x, v_{1}, v_{2}\right) \mid x=1, v_{1} \neq 0, v_{2} \in \mathbf{k} v_{1}\right\}$. In particular, $X_{\boldsymbol{\mu}} \cap \bar{X}_{\boldsymbol{\lambda}} \neq \emptyset$ and $X_{\boldsymbol{\mu}} \not \subset \bar{X}_{\boldsymbol{\lambda}}$.

The following result is an analogue of [AH, Cor. 3.4].

Proposition 5.16. Let $(x, \mathbf{v}) \in \mathcal{X}_{\text {uni }}$, and $\boldsymbol{\lambda}, \boldsymbol{\xi} \in \mathcal{P}(\mathbf{m})$ for some $\mathbf{m}$. Then $(x, \mathbf{v}) \in$ $\bar{X}_{\boldsymbol{\lambda}}$ if and only if there exists a flag $\left(W_{i}\right)_{1 \leq i \leq r}$ in $V$ such that $\operatorname{dim} W_{i}=p_{i}$ satisfying the following conditions;

(i) $\left(W_{i}\right)$ is x-stable,

(ii) $\left.x\right|_{W_{i} / W_{i-1}}$ has type $\xi^{(i)}$ with $\xi^{(i)} \leq \lambda^{(i)}$ for $1 \leq i \leq r$,

(iii) $v_{i} \in W_{i}$ for $1 \leq i \leq r-1$.

Proof. Let $\mathcal{U}$ be the set of $(x, \mathbf{v})$ satisfying the condition in the proposition. We show that $\mathcal{U}=\bar{X}_{\boldsymbol{\lambda}}$. Take $(x, \mathbf{v}) \in \mathcal{U}$. In order to show that $(x, \mathbf{v}) \in \bar{X}_{\boldsymbol{\lambda}}$, by Lemma 5.6 , it is enough to construct $\left(x, \mathbf{v}, g P_{\boldsymbol{\lambda}}\right) \in \widetilde{X}_{\boldsymbol{\lambda}}$. Under the notation in 5.5, put $V_{n(i, 0)}=W_{i}$ for $i=1, \ldots, r-1$. By assumption, the restriction of $x$ on $W_{i} / W_{i-1}$ has type $\xi^{(i)}$. 
Since $\xi^{(i)} \leq \lambda^{(i)}$, there exists an $x$-stable flag

$$
W_{i-1}=V_{n(i-1,0)} \subset V_{n(i-1,1)} \subset \cdots \subset V_{n(i, 0)}=W_{i}
$$

such that $(x-1) V_{n(i, j)} \subset V_{n(i, j-1)}$. In fact this is an application of Lemma 5.6 for $r=1$, which is a well-konwn result for $G L_{n}$. There exists $g \in G$ such that $g\left(M_{n(i, j)}\right)=\left(V_{n(i, j)}\right)$, and $\left(x, \mathbf{v}, g P_{\boldsymbol{\lambda}}\right) \in \tilde{X}_{\boldsymbol{\lambda}}$. Hence $(x, \mathbf{v}) \in \bar{X}_{\boldsymbol{\lambda}}$, and we have $\mathcal{U} \subset \bar{X}_{\lambda}$.

Let $P$ be a parabolic subgroup of $G$ which is the stabilizer of a partial flag $\left(M_{p_{i}}\right)_{1 \leq i \leq r}$, and $L$ the Levi subgroup of $P$ containing $T$. Thus $L \simeq \prod_{i} G L_{m_{i}}$. Let $\mathcal{O}_{\boldsymbol{\lambda}}^{\prime}$ be the $L$-orbit in $L_{\text {uni }}$ corresponding to $\boldsymbol{\lambda}=\left(\lambda^{(1)}, \ldots, \lambda^{(r)}\right)$, and $\overline{\mathcal{O}}_{\boldsymbol{\lambda}}^{\prime}$ the closure of $\mathcal{O}_{\lambda}^{\prime}$ in $L_{\text {uni }}$. Let $p: P \rightarrow L$ be the natural projection. We consider a variety

$$
\widetilde{\mathcal{P}}_{\mathcal{O}_{\boldsymbol{\lambda}}^{\prime}}=\left\{(x, \mathbf{v}, g P) \in G_{\text {uni }} \times V^{r-1} \times G / P \mid g^{-1} x g \in p^{-1}\left(\overline{\mathcal{O}}_{\lambda}^{\prime}\right), g^{-1} v_{i} \in M_{p_{i}}\right\},
$$

and let $f: \widetilde{\mathcal{P}}_{\mathcal{O}_{\lambda}^{\prime}} \rightarrow G_{\text {uni }} \times V^{r-1}$ be the projection on the first two factors. Then $\operatorname{Im} f=\mathcal{U}$. Since $f$ is proper, $\mathcal{U}$ is a closed subvariety of $G_{\text {uni }} \times V^{r-1}$. The construction of $X_{\boldsymbol{\lambda}}$ implies that $X_{\boldsymbol{\lambda}} \subset \mathcal{U}$. It follows that $\bar{X}_{\boldsymbol{\lambda}} \subset \mathcal{U}$, and so $\mathcal{U}=\bar{X}_{\boldsymbol{\lambda}}$. The proposition is proved.

As a corollary, we have the following result.

Corollary 5.17. (i) Let $\mathcal{U}_{\mathcal{O}_{\lambda}^{\prime}}$ be the set of $(x, \mathbf{v}) \in G_{\text {uni }} \times V^{r-1}$ satisfying the condition; there exists a flag $\left(W_{i}\right)_{1 \leq i \leq r}$ in $V$ such that $\operatorname{dim} W_{i}=p_{i}$ and that

(a) $\left(W_{i}\right)$ is $x$-stable,

(b) $\left.x\right|_{W_{i} / W_{i-1}}$ has type $\lambda^{(i)}$,

(c) $v_{i} \in W_{i}$ for $i=1, \ldots, r-1$. Then

$$
\mathcal{U}_{\mathcal{O}_{\lambda}^{\prime}}=\bar{X}_{\lambda} \backslash \bigcup_{\mu} \bar{X}_{\mu}
$$

wehere $\boldsymbol{\mu}$ runs over elements in $\mathcal{P}_{n, r}$ such that $\mu^{(i)} \leq \lambda^{(i)}$ for any $i$ and that $\boldsymbol{\mu} \neq \boldsymbol{\lambda}$.

(ii) $X_{\boldsymbol{\lambda}}$ coincides with the set of $(x, \mathbf{v}) \in \mathcal{U}_{\mathcal{O}_{\boldsymbol{\lambda}}^{\prime}}$ such that the Jordan type of $x$ is $\lambda^{(1)}+\cdots+\lambda^{(r)}$ and that the type of $\left(\left.x\right|_{W_{i} / W_{i-1}}, \bar{v}_{i}\right)$ is $\left(\lambda^{(i)}, \emptyset\right)$ for each $i$, where $\bar{v}_{i}$ is the image of $v_{i}$ to $W_{i} / W_{i-1}$.

Proof. (i) is clear from Proposition 5.16. By induction on $r$, the proof of (ii) is reduced to the case where $r=2$. In that case, the assertion easily follows from the discuusion by using the normal basis $[\mathrm{AH}, 2.2]$.

5.18. Take $\mathbf{m} \in \mathcal{Q}_{n, r}$. For each $z=(x, \mathbf{v}) \in \mathcal{X}_{\mathbf{m} \text {,uni, }}$ put

$$
\mathcal{B}_{z}^{(\mathbf{m})}=\left\{g B \in G / B \mid g^{-1} x g \in U, g^{-1} \mathbf{v} \in \prod_{i=1}^{r-1} M_{p_{i}}\right\}
$$


$\mathcal{B}_{z}^{(\mathbf{m})}$ is a closed subvariety of $\mathcal{B}=G / B$, which is isomorphic to the fibre $\left(\pi_{1}^{(\mathbf{m})}\right)^{-1}(z)$ and is called the Springer fibre of $z$. In the case where $r=2, \mathcal{B}_{z}^{(\mathbf{m})}$ is isomorphic each other for any $z \in X_{\boldsymbol{\lambda}}$ since $X_{\boldsymbol{\lambda}}$ is a single $G$-orbit. However this property does not hold in general for $r \geq 3$ as the next examples show. First assume that $r=3$ and $n=3$. Let $\mathbf{m}=(2,0,1)$ and $\boldsymbol{\lambda}=\left(\left(1^{3}\right),-,-\right)$. Let $v_{1}, v_{2}$ be linearly independent vectors in $V$, and take $z=\left(x,\left(v_{1}, v_{2}\right)\right), z^{\prime}=\left(x,\left(v_{1}, 0\right)\right)$ with $x=1$. Then $z, z^{\prime} \in X_{\boldsymbol{\lambda}}$. Let $\mathcal{F}(V)$ be the set of total flags $\left(V_{i}\right)$ in $V$. We have

$$
\begin{aligned}
& \mathcal{B}_{z}^{(\mathbf{m})} \simeq\left\{\left(V_{i}\right) \in \mathcal{F}(V) \mid V_{2}=\left\langle v_{1}, v_{2}\right\rangle\right\} \\
& \mathcal{B}_{z^{\prime}}^{(\mathbf{m})} \simeq\left\{\left(V_{i}\right) \in \mathcal{F}(V) \mid v_{1} \in V_{2}\right\} .
\end{aligned}
$$

Hence $\operatorname{dim} \mathcal{B}_{z}^{(\mathbf{m})}=1, \operatorname{dim} \mathcal{B}_{z^{\prime}}^{(\mathbf{m})}=2$, and in particular, $z, z^{\prime} \in X_{\boldsymbol{\lambda}} \cap \mathcal{X}_{\mathbf{m} \text {,uni }}$. We have $\operatorname{dim} \mathcal{B}_{z}^{(\mathbf{m})} \neq \operatorname{dim} \mathcal{B}_{z^{\prime}}^{(\mathbf{m})}$

Next assume that $r=3$ and $n=4$. Let $\mathbf{m}=(2,0,2)$ and $\boldsymbol{\lambda}=\left(\left(1^{2}\right),-,\left(1^{2}\right)\right)$. We have $X_{\boldsymbol{\lambda}} \subset \mathcal{X}_{\mathbf{m} \text {,uni }}$. Let $x$ be an element in $G_{\text {uni }}$ of type $\left(2^{2}\right)$, and $\left\{u_{i, j} \mid 1 \leq i \leq\right.$ $2,1 \leq j \leq 2\}$ be a Jordan baasis of $x$ in $V$. Put $v_{1}=u_{1,1}, v_{2}=u_{2,1}$ so that $v_{1}, v_{2}$ is a basis of $W=\operatorname{Ker}(x-1)$. Put $z=\left(x,\left(v_{1}, v_{2}\right)\right)$ and $z^{\prime}=\left(x,\left(v_{1}, 0\right)\right)$. Then $z, z^{\prime} \in X_{\boldsymbol{\lambda}}$. We have

$$
\begin{aligned}
& \mathcal{B}_{z}^{(\mathbf{m})} \simeq\left\{\left(V_{i}\right) \in \mathcal{F}_{x}(V) \mid V_{2}=W\right\} \\
& \mathcal{B}_{z^{\prime}}^{(\mathbf{m})} \simeq\left\{\left(V_{i}\right) \in \mathcal{F}_{x}(V) \mid v_{1} \in V_{2}\right\}
\end{aligned}
$$

where $\mathcal{F}_{x}(V)$ is the set of $x$-stable flags in $V$. Since $\left.x\right|_{W}=1, x_{V / W}=1$, we see that $\mathcal{B}_{z}^{(\mathbf{m})} \simeq \mathcal{F}(W) \times \mathcal{F}(V / W)$. Hnece $\mathcal{B}_{z}^{(\mathbf{m})}$ is irreducible with $\operatorname{dim} \mathcal{B}_{z}^{(\mathbf{m})}=2$. On the other hand, if $\left(V_{i}\right) \in \mathcal{B}_{z^{\prime}}^{(\mathbf{m})}$, either $V_{2}=W$ or $V_{2}$ is of the form $W_{\alpha}=\left\langle u_{1,2}+\alpha v_{2}, v_{1}\right\rangle$ for $\alpha \in \mathbf{k}$. Since $\left.x\right|_{W_{\alpha}}$ and $\left.x\right|_{V / W_{\alpha}}$ have both type (2), $W_{\alpha}$ determines a unique $\left(V_{i}\right) \in \mathcal{B}_{z^{\prime}}^{(\mathbf{m})}$. It follows that $\mathcal{B}_{z^{\prime}}^{(\mathbf{m})}=\mathcal{B}_{z}^{(\mathbf{m})} \coprod Y$ with $Y=\left\{\left(V_{i}\right) \mid V_{2}=W_{\alpha}(\alpha \in \mathbf{k})\right\}$, where $Y$ is irreducible with $\operatorname{dim} Y=1$. Hence in this case, $\operatorname{dim} \mathcal{B}_{z}^{(\mathbf{m})}=\operatorname{dim} \mathcal{B}_{z^{\prime}}^{(\mathbf{m})}$, but $\mathcal{B}_{z}^{(\mathbf{m})} \not \mathcal{B}_{z^{\prime}}^{(\mathbf{m})}$.

\section{UNIPOTENT VARIETY OF EXOTIC TYPE}

The "unipotent part" of the exotic space is called the unipotent variety of exotic type. In this section, first we study the unipotent variety of exotic type in $6.1-6.13$. While in $6.14-6.19$, we discuss the case of enahnced type. After 6.20, we disucss the both cases simultaneously. 
6.1. We follow the notation in 1.2. Assume that $\mathcal{X}$ is of exotic type. As in the enhanced case we define varieties, for each $\mathbf{m} \in \mathcal{Q}_{n, r}$,

$$
\begin{aligned}
& \widetilde{\mathcal{X}}_{\mathbf{m}, \text { uni }}=\left\{\left(x, \mathbf{v}, g B^{\theta}\right) \in G_{\text {uni }}^{\iota \theta} \times V^{r-1} \times H / B^{\theta} \mid g^{-1} x g \in U^{\iota \theta}, g^{-1} \mathbf{v} \in \prod_{i=1}^{r-1} M_{p_{i}}\right\}, \\
& \mathcal{X}_{\mathbf{m}, \text { uni }}=\bigcup_{g \in H} g\left(U^{\iota \theta} \times \prod_{i=1}^{r-1} M_{p_{i}}\right),
\end{aligned}
$$

where $B, T$ are as in 1.2 , and $U$ is the unipotent radical of $B$. We define a map $\pi_{1}^{(\mathbf{m})}: \widetilde{\mathcal{X}}_{\mathbf{m} \text {,uni }} \rightarrow G_{\text {uni }}^{\iota \theta} \times V^{r-1}$ by $\pi_{1}^{(\mathbf{m})}\left(x, \mathbf{v}, g B^{\theta}\right)=(x, \mathbf{v})$. Clearly $\mathcal{X}_{\mathbf{m}, \text { uni }}=\operatorname{Im} \pi_{1}^{(\mathbf{m})}$. As in 1.2 , in the case where $\mathbf{m}=(n, 0, \ldots, 0)$, we write $\widetilde{\mathcal{X}}_{\mathbf{m} \text {,uni }}, \mathcal{X}_{\mathbf{m} \text {,uni }}, \pi_{1}^{(\mathbf{m})}$, etc. by $\widetilde{\mathcal{X}}_{\text {uni }}, \mathcal{X}_{\text {uni }}, \pi_{1}$, etc. Note that $\mathcal{X}_{\mathbf{m} \text {,uni }} \subset \mathcal{X}_{\text {uni }}$ for any $\mathbf{m}$, but contrast to the enhanced case, $\mathcal{X}_{\text {uni }}$ does not coincide with $G_{\text {uni }}^{\iota \theta} \times V^{r-1}$ if $r \geq 3$. The variety $\mathcal{X}_{\text {uni }}$ is called a unipotent variety of exotic type.

Since $\widetilde{\mathcal{X}}_{\mathbf{m} \text {,uni }} \simeq H \times{ }^{B^{\theta}}\left(U^{\imath \theta} \times \prod_{i} M_{p_{i}}\right), \widetilde{\mathcal{X}}_{\mathbf{m} \text {,uni }}$ is smooth and irreducible. Moreover, we have

$$
\begin{aligned}
\operatorname{dim} \widetilde{\mathcal{X}}_{\mathbf{m}, \text { uni }} & =\operatorname{dim} H / B^{\theta}+\left(\operatorname{dim} U^{\iota \theta}+\operatorname{dim} \prod_{i=1}^{r-1} M_{p_{i}}\right) \\
& =\operatorname{dim} U^{\theta}+\operatorname{dim} U^{\iota \theta}+\sum_{i=1}^{r-1} p_{i} \\
& =2 n^{2}-n+\sum_{i=1}^{r-1}(r-i) m_{i}
\end{aligned}
$$

since $\operatorname{dim} U^{\theta}+\operatorname{dim} U^{\iota \theta}=\operatorname{dim} U$. Since $\pi_{1}^{(\mathbf{m})}$ is proper, $\mathcal{X}_{\mathbf{m} \text {,uni }}$ is a closed irreducible subvariety of $G_{\mathrm{uni}}^{\iota \theta} \times V^{r-1}$.

6.2. Assume that $r \geq 2$, and take $\mathbf{m} \in \mathcal{Q}_{n, r}$. Let $P$ be the $\theta$-stable parabolic subgroup of $G$ such that $P^{\theta}$ is the stabilizer of the isotropic flag $\left(M_{p_{i}}\right)_{1 \leq i \leq r-2}$ in $V$. Let $L$ be the $\theta$-stable Levi subgroup of $P$ containing $T$ and $U_{P}$ the unipotent radical of $P$. Put $V_{L}^{\prime}=M_{p_{r-2}}$, and $V_{L}=V / V_{L}^{\prime}$. Then $P^{\theta}$ acts naturally on $V_{L}$, and we consider the map $\pi_{P}: P_{\text {uni }}^{\iota \theta} \times V \rightarrow L_{\text {uni }}^{\iota \theta} \times V_{L},(x, v) \mapsto\left(x^{\prime}, v^{\prime}\right)$, where $x^{\prime}=p(x)$ for the natural projection $p: P^{\iota \theta} \rightarrow L^{\iota \theta}$, and $v \mapsto v^{\prime}$ is the projection $V \rightarrow V_{L}=V / V_{L}^{\prime}$. The map $\pi_{P}$ is $P^{\theta}$-equivariant with respect to the diagonal action of $P^{\theta}$ on both varieties.

Let $\mathcal{O}^{\prime}$ be an $L^{\theta}$-orbit in $L_{\text {uni }}^{\iota \theta} \times V_{L}$. We assume that $\mathcal{O}^{\prime}$ is $P^{\theta}$-stable. Since $\pi_{P}^{-1}\left(\mathcal{O}^{\prime}\right)=\bigcup_{g \in P^{\theta}} g \cdot \pi_{P}^{-1}\left(z^{\prime}\right)$ for $z^{\prime} \in \mathcal{O}^{\prime}, \pi_{P}^{-1}\left(\mathcal{O}^{\prime}\right)$ is irreducible (note that $\pi_{P}^{-1}\left(z^{\prime}\right)$ is irreducible). Let $\mathcal{O}$ be an $H$-orbit in $G_{\text {uni }}^{\iota \theta} \times V$ such that $\mathcal{O} \cap \pi_{P}^{-1}\left(\mathcal{O}^{\prime}\right)$ is open dense in $\pi_{P}^{-1}\left(\mathcal{O}^{\prime}\right)$. Put

$$
\mathcal{U}=\left\{(z, g P) \in\left(G_{\text {uni }}^{\iota \theta} \times V\right) \times H / P^{\theta} \mid g^{-1} z \in \pi_{P}^{-1}\left(\mathcal{O}^{\prime}\right)\right\}
$$


Then $\mathcal{U} \simeq H \times{ }^{P^{\theta}} \pi_{P}^{-1}\left(\mathcal{O}^{\prime}\right)$ and so $\mathcal{U}$ is an irreducible variety. Let $f: \mathcal{U} \rightarrow G_{\text {uni }}^{\iota \theta} \times V$ be the first projection, and put $\mathcal{U}_{\mathcal{O}}=f^{-1}(\mathcal{O})$. Then $\mathcal{U}_{\mathcal{O}} \simeq H \times{ }^{P^{\theta}}\left(\mathcal{O} \cap \pi_{P}^{-1}\left(\mathcal{O}^{\prime}\right)\right)$, and so $\mathcal{U}_{\mathcal{O}}$ is irreducible.

Take $z \in \mathcal{O}$, and consider a variety

$$
\mathcal{P}_{z, \mathcal{O}^{\prime}}=\left\{g P^{\theta} \in H / P^{\theta} \mid g^{-1} z \in \pi_{P}^{-1}\left(\mathcal{O}^{\prime}\right)\right\}
$$

Note that $\mathcal{P}_{z, \mathcal{O}^{\prime}} \neq \emptyset$. We show the following proposition.

Proposition 6.3. Under the setting in 6.2, the followings hold.

(i) $\mathcal{P}_{z, \mathcal{O}^{\prime}}$ consists of one point.

(ii) $\operatorname{dim} Z_{H}(z)=\operatorname{dim} Z_{L^{\theta}}\left(z^{\prime}\right)$ for $z^{\prime} \in \mathcal{O}^{\prime}$.

(iii) Let $z_{1} \in \pi_{P}^{-1}\left(\mathcal{O}^{\prime}\right)$ be such that $\operatorname{dim} Z_{H}\left(z_{1}\right)=\operatorname{dim} Z_{H}(z)$. Then $z_{1} \in \mathcal{O}$.

(iv) Take $z \in \mathcal{O} \cap \pi_{P}^{-1}\left(\mathcal{O}^{\prime}\right)$ and put $z^{\prime}=\pi_{P}(z)$. Let $Q=Z_{P}\left(z^{\prime}\right)$ be a $\theta$-stable subgroup of $P$. Then $\operatorname{dim} Z_{Q^{\theta}}(z)=\operatorname{dim} Z_{H}(z)$. In particular,

$$
Z_{H}(z)=Z_{P^{\theta}}(z)=Z_{Q^{\theta}}(z)
$$

(v) $H$ acts transitively on $\mathcal{U}_{\mathcal{O}}$.

(vi) $P^{\theta}$ acts transitively on $\mathcal{O} \cap \pi_{P}^{-1}\left(\mathcal{O}^{\prime}\right)$, and $Q^{\theta}$ acts transitively on $\mathcal{O} \cap \pi_{P}^{-1}\left(z^{\prime}\right)$ under the setting in (iv).

Proof. First we show that

$$
\operatorname{dim} \mathcal{P}_{z, \mathcal{O}^{\prime}}=0
$$

Replacing $z$ and $\mathcal{P}_{z, \mathcal{O}^{\prime}}$ by $H$-conjugate, if necessary, we may assume that $z \in \mathcal{O} \cap$ $\pi_{P}^{-1}\left(\mathcal{O}^{\prime}\right)$. Put $z^{\prime}=\left(x^{\prime}, v^{\prime}\right)=\pi_{P}(z)$. Since $\pi_{P}^{-1}\left(z^{\prime}\right)=\left(x^{\prime} U_{P}\right)^{\iota \theta} \times\left(v^{\prime}+V_{L}^{\prime}\right) \simeq U_{P}^{\iota \theta} \times V_{L}^{\prime}$, we have

$$
\operatorname{dim} \pi_{P}^{-1}\left(z^{\prime}\right)=\operatorname{dim} U_{P}^{\iota \theta}+\operatorname{dim} V_{L}^{\prime}=\operatorname{dim} U_{P}^{\theta}
$$

Put $c=\operatorname{dim} \mathcal{O}$ and $c^{\prime}=\operatorname{dim} \mathcal{O}^{\prime}$. Then by [SS11, Prop. 5.7 (i)] (for a correction, see $[\mathrm{SS} 2$, Appendix] $)$, we have $\operatorname{dim}\left(\mathcal{O} \cap \pi_{P}^{-1}\left(z^{\prime}\right)\right) \leq\left(c-c^{\prime}\right) / 2$. Since $\mathcal{O} \cap \pi_{P}^{-1}\left(\mathcal{O}^{\prime}\right)$ is open dense in $\pi_{P}^{-1}\left(\mathcal{O}^{\prime}\right), \mathcal{O} \cap \pi_{P}^{-1}\left(z^{\prime}\right)$ is open dense in $\pi_{P}^{-1}\left(z^{\prime}\right)$. It follows that

$$
\operatorname{dim} U_{P}^{\theta} \leq\left(c-c^{\prime}\right) / 2
$$

On the other hand, by [SS1, Prop. 5.7 (ii)] together with [SS2, Appendix], we have

$$
\begin{aligned}
\operatorname{dim} \mathcal{P}_{z, \mathcal{O}^{\prime}} & \leq\left(\nu_{H}-c / 2\right)-\left(\nu_{L^{\theta}}-c^{\prime} / 2\right) \\
& =\operatorname{dim} U^{\theta}-\operatorname{dim} U_{L}^{\theta}-\left(c-c^{\prime}\right) / 2 \\
& =\operatorname{dim} U_{P}^{\theta}-\left(c-c^{\prime}\right) / 2 .
\end{aligned}
$$

Hence $\operatorname{dim} \mathcal{P}_{z, \mathcal{O}^{\prime}} \leq 0$. As $\mathcal{P}_{z, \mathcal{O}^{\prime}} \neq \emptyset$, we obtain (6.3.1).

Substituting this into (6.3.3), we have $c-c^{\prime}=2 \operatorname{dim} U_{P}^{\theta}$. This implies that $\operatorname{dim} Z_{H}(z)=\operatorname{dim} Z_{L^{\theta}}\left(z^{\prime}\right)$. Hence (ii) holds. 
Under the setting in (iv), $Q^{\theta}$ stabilizes $\pi_{P}^{-1}\left(z^{\prime}\right)$, and so $\mathcal{O}_{Q} \subset \mathcal{O} \cap \pi_{P}^{-1}\left(z^{\prime}\right)$. Since $\mathcal{O}^{\prime}$ is an $P^{\theta}$-orbit, we have $\operatorname{dim} Z_{P^{\theta}}\left(z^{\prime}\right)=\operatorname{dim} Z_{L^{\theta}}\left(z^{\prime}\right)+\operatorname{dim} U_{P}^{\theta}$. Hence by (6.3.2) we have

$$
\operatorname{dim} U_{P}^{\theta} \geq \operatorname{dim} \mathcal{O}_{Q}=\operatorname{dim} Z_{L^{\theta}}\left(z^{\prime}\right)+\operatorname{dim} U_{P}^{\theta}-\operatorname{dim} Z_{Q^{\theta}}(z) .
$$

It follows that $\operatorname{dim} Z_{Q^{\theta}}(z) \geq \operatorname{dim} Z_{L^{\theta}}\left(z^{\prime}\right)$. Since $\operatorname{dim} Z_{Q^{\theta}}(z) \leq \operatorname{dim} Z_{H}(z)$, we have $\operatorname{dim} Z_{Q^{\theta}}(z)=\operatorname{dim} Z_{H}(z)$ by (6.3.4). Since $Z_{H}(z)$ is connected, this implies that $Z_{H}(z)=Z_{Q^{\theta}}(z)$. Hence $Z_{H}(z)=Z_{P^{\theta}}(z)$. Thus (iv) holds.

For any $z \in \mathcal{O}, \operatorname{dim} f^{-1}(z)=0$ by (6.3.1). Thus $\operatorname{dim} \mathcal{U}_{\mathcal{O}}=\operatorname{dim} \mathcal{O}$. We show that $H$ acts transitively on $\mathcal{U}_{\mathcal{O}}$. Take $\xi \in \mathcal{U}_{\mathcal{O}}$, and consider the $H$-orbit $H \xi$ of $\xi$. Since $f$ is $H$-equivariant, $f$ maps $H \xi$ onto $\mathcal{O}$. Hence $H \xi$ is irreducible with $\operatorname{dim} H \xi=\operatorname{dim} \mathcal{U}_{\mathcal{O}}$. In particular $H \xi$ is open dense in $\mathcal{U}_{\mathcal{O}}$. If we take another $\xi^{\prime} \in \mathcal{U}_{\mathcal{O}}, H \xi^{\prime}$ is also open dense in $\mathcal{U}_{\mathcal{O}}$. Hence $H \xi \cap H \xi^{\prime} \neq \emptyset$. Thus $\mathcal{U}_{\mathcal{O}}=H \xi$ and (v) holds.

Take $z, z_{1} \in \mathcal{O} \cap \pi_{P}^{-1}\left(\mathcal{O}^{\prime}\right)$. Since $\left(z, P^{\theta}\right),\left(z_{1}, P^{\theta}\right)$ are both contained in $\mathcal{U}_{\mathcal{O}}$, they are in the same $H$-orbit by (v). Hence there exists $g \in P^{\theta}$ such that $g z=z_{1}$. This proves the first statement of (vi). Then for $z_{1}, z_{2} \in \mathcal{O} \cap \pi_{P}^{-1}\left(z^{\prime}\right)$, there exists $p \in P^{\theta}$ such that $p z_{1}=z_{2}$. But since $\pi_{P}$ is $P^{\theta}$-equivariant, $p \in Z_{P^{\theta}}\left(z^{\prime}\right)=Q^{\theta}$. This proves the second statement of (vi).

We show (i). We may assume that $z \in \mathcal{O} \cap \pi_{P}^{-1}\left(\mathcal{O}^{\prime}\right)$. Then $P^{\theta} \in \mathcal{P}_{z, \mathcal{O}^{\prime}}$. Assume that $g P^{\theta} \in \mathcal{P}_{z, \mathcal{O}^{\prime}}$. Then $\left(z, P^{\theta}\right),\left(z, g P^{\theta}\right)$ are both contained in $\mathcal{U}_{\mathcal{O}}$. By $(\mathrm{v})$ they are conjugate under $H$. It follows that there exists $h \in Z_{H}(z)$ such that $g P^{\theta}=h P^{\theta}$. But by (iv), we have $h \in P^{\theta}$, and so $g P^{\theta}=P^{\theta}$. This proves (i).

Finally we show (iii). Let $\mathcal{O}_{1}$ be the $H$-orbit of $z_{1}$. Then $\operatorname{dim} \mathcal{O}_{1}=c$. By a similar argument as in the proof of (6.3.1), we have $\operatorname{dim} \mathcal{P}_{z_{1}, \mathcal{O}^{\prime}}=0$. Then a similar argument as in the proof of (iv) implies that $Z_{H}\left(z_{1}\right)=Z_{Q^{\theta}}\left(z_{1}\right)$. Hence $\operatorname{dim} Z_{Q^{\theta}}(z)=\operatorname{dim} Z_{Q^{\theta}}\left(z_{1}\right)$. This shows that the $Q^{\theta}$-orbit of $z_{1}$ in $\pi_{P}^{-1}\left(z^{\prime}\right)$ has the same dimension as the $Q^{\theta}$-orbit of $z$. Since both orbits are open dense in $\pi_{P}^{-1}\left(z^{\prime}\right)$, they have an intersection. Hence $\mathcal{O}=\mathcal{O}_{1}$. This proves (iii). The proposition is proved.

6.4. Let $P$ be as in 6.2. For $\boldsymbol{\lambda} \in \mathcal{P}(\mathbf{m})$, we define a subset $\mathcal{M}_{\boldsymbol{\lambda}}$ of $P_{\text {uni }}^{\iota \theta} \times$ $\left(\prod_{i=1}^{r-2} M_{p_{i}} \times M_{p_{r-2}}^{\perp}\right)$ as the set of $(x, \mathbf{v})$ satisfying the following properties; take $(x, \mathbf{v})$ such that $x \in P_{\text {uni }}^{\iota \theta}$ and that $v_{i} \in M_{p_{i}}$ for $i=1, \ldots, r-2, v_{r-1} \in M_{p_{r-2}}^{\perp}$. Put $\bar{M}_{p_{i}}=M_{p_{i}} / M_{p_{i-1}}$, and let $\bar{v}_{i} \in \bar{M}_{p_{i}}$ be the image of $v_{i} \in M_{p_{i}}$ for $i=1, \ldots, r-2$. We also put $\bar{M}_{p_{r-1}}^{\prime}=M_{p_{r-2}}^{\perp} / M_{p_{r-2}}$. Thus $\bar{M}_{p_{r-1}}^{\prime}$ has a structure of a symplectic vector space, and one can consider an exotic symmetric space $G L\left(\bar{M}_{p_{r-1}}^{\prime}\right)^{\iota \theta} \times \bar{M}_{p_{r-1}}^{\prime}$. We also consider enhanced spaces $G L\left(\bar{M}_{p_{i}}\right) \times \bar{M}_{p_{i}}$ for $i=1, \ldots, r-2$. Let $\bar{v}_{r-1}$ be the image of $v_{r-1}$ on $\bar{M}_{p_{r-1}}^{\prime}$. We assume that the $G L\left(\bar{M}_{p_{i}}\right)$-orbit of $\left(\left.x\right|_{M_{p_{i}}}, \bar{v}_{i}\right)$ has type $\left(\lambda^{(i)}, \emptyset\right)$ for $i=1, \ldots, r-2$, and that the $G L\left(\bar{M}_{p_{r-1}}^{\prime}\right)^{\theta}$-orbit of $\left(\left.x\right|_{\bar{M}_{p_{r-1}}^{\prime}}, \bar{v}_{r-1}\right)$ has type $\left(\lambda^{(r-1)}, \lambda^{(r)}\right)$. Let $\mathcal{O}$ be the $H$-orbit of $z=\left(x, v_{r-1}\right) \in G_{\text {uni }}^{\iota \theta} \times V$ and $\mathcal{O}^{\prime}$ the $L^{\theta}$-orbit of $z^{\prime}=\pi_{P}(z)$. Note that $\mathcal{O}^{\prime}$ is $P^{\theta}$-stable. We further assume that

$$
\mathcal{O} \cap \pi_{P}^{-1}\left(\mathcal{O}^{\prime}\right) \text { is open dense in } \pi_{P}^{-1}\left(\mathcal{O}^{\prime}\right) \text {. }
$$


We define $X_{\boldsymbol{\lambda}}$ by $X_{\boldsymbol{\lambda}}=\bigcup_{g \in H} g \mathcal{M}_{\boldsymbol{\lambda}}$. Note that in the case where $r=2, X_{\boldsymbol{\lambda}}$ coincides with the $H$-orbit $\mathcal{O}_{\boldsymbol{\lambda}}$ in $[\mathrm{SS} 1]$.

It follows from the construction that $\mathcal{M}_{\boldsymbol{\lambda}}$ is a $P^{\theta}$-stable subset of $P^{\iota \theta} \times V^{r-1}$. The closure $\overline{\mathcal{M}}_{\boldsymbol{\lambda}}$ of $\mathcal{M}_{\boldsymbol{\lambda}}$ in $P^{\iota \theta} \times V^{r-1}$ is also $P^{\theta}$-stable. We define a variety $\widetilde{\mathcal{F}}_{\boldsymbol{\lambda}}$ by $\widetilde{\mathcal{F}}_{\boldsymbol{\lambda}}=H \times{ }^{P^{\theta}} \overline{\mathcal{M}}_{\boldsymbol{\lambda}}$. Let $\pi_{\boldsymbol{\lambda}}: \widetilde{\mathcal{F}}_{\boldsymbol{\lambda}} \rightarrow \mathcal{X}_{\text {uni }}$ be the map induced from the map $H \times \overline{\mathcal{M}}_{\boldsymbol{\lambda}} \rightarrow \mathcal{X}_{\text {uni }},(g, z) \mapsto g \cdot z$. Then $\pi_{\boldsymbol{\lambda}}$ is a proper map. We define a subset $\widetilde{\mathcal{F}}_{\boldsymbol{\lambda}}^{0}$ of $\widetilde{\mathcal{F}}_{\boldsymbol{\lambda}}$ by $\widetilde{\mathcal{F}}_{\boldsymbol{\lambda}}^{0}=H \times{ }^{P^{\theta}} \mathcal{M}_{\boldsymbol{\lambda}}$, and let $\pi_{\boldsymbol{\lambda}}^{0}$ be the restriction of $\pi_{\boldsymbol{\lambda}}$ on $\widetilde{\mathcal{F}}_{\boldsymbol{\lambda}}^{0}$. It is clear that $\pi_{\lambda}^{0}$ is a surjective map onto $X_{\boldsymbol{\lambda}}$.

6.5. Take $x_{i} \in G L\left(\bar{M}_{p_{i}}\right)$ uni with Jordan type $\lambda^{(i)}$ for $1 \leq i \leq r-2$. Then the set of $\bar{v}_{i} \in \bar{M}_{p_{i}}$ such that $\left(x_{i}, \bar{v}_{i}\right)$ is of type $\left(\lambda^{(i)}, \emptyset\right)$ is open dense in $\bar{M}_{p_{i}}$. Take $\left(x_{r-1}, \bar{v}_{r-1}\right) \in G L\left(\bar{M}_{p_{r-1}}^{\prime}\right)_{\text {uni }}^{\iota \theta} \times \bar{M}_{p_{r-1}}^{\prime}$ with type $\left(\lambda^{(r-1)}, \lambda^{(r)}\right)$. Now $\left(x_{1}, \ldots, x_{r-1}\right)$ determines a unique element in $L_{\text {uni }}^{\iota \theta}$ which we denote by $x^{\prime}$. Put $z^{\prime}=\left(x^{\prime}, \bar{v}_{r-1}\right)$. The set of $\left(x, v_{r-1}\right) \in G_{\mathrm{uni}}^{\iota \theta} \times V$ such that $\left(x, v_{r-1}\right) \in \pi_{P}^{-1}\left(z^{\prime}\right)$ satisfying the condition (6.4.1) is open dense in $\pi_{P}^{-1}\left(z^{\prime}\right)$. In fact, it coincides with $\mathcal{O} \cap \pi_{P}^{-1}\left(z^{\prime}\right)$ for an $H$-orbit $\mathcal{O}$. Put $\mathbf{v}^{\prime}=\left(\bar{v}_{1}, \ldots, \bar{v}_{r-1}\right)$. For a fixed $\left(x^{\prime}, \mathbf{v}^{\prime}\right)$, let $\mathcal{M}_{\left(x^{\prime}, \mathbf{v}^{\prime}\right)}$ be the set of $(x, \mathbf{v})$ with $\mathbf{v}=\left(v_{1}, \ldots, v_{r-1}\right)$ such that $\left(x, v_{r-1}\right) \in \mathcal{O} \cap \pi_{P}^{-1}\left(z^{\prime}\right)$ as in (6.4.1) and that the image of $v_{i}$ on $\bar{M}_{p_{i}}$ coincides with $\bar{v}_{i}$. As $\mathcal{M}_{\left(x^{\prime}, \mathbf{v}^{\prime}\right)}$ is an open dense subset of $\prod_{i=1}^{r-3} M_{p_{i}} \times \pi_{P}^{-1}\left(z^{\prime}\right)$, we have

$$
\operatorname{dim} \mathcal{M}_{\left(x^{\prime}, \mathbf{v}^{\prime}\right)}=\sum_{i=1}^{r-3} \operatorname{dim} M_{p_{i}}+\operatorname{dim} \pi_{P}^{-1}\left(z^{\prime}\right) .
$$

It follows that $\mathcal{M}_{\left(x^{\prime}, \mathbf{v}^{\prime}\right)}$ is smooth irreducible, and

$$
\operatorname{dim} \mathcal{M}_{\left(x^{\prime}, \mathbf{v}^{\prime}\right)}=\sum_{i=1}^{r-3}(r-i-2) m_{i}+\operatorname{dim} U_{P}^{\theta}
$$

(Note $\operatorname{dim} \pi_{P}^{-1}\left(z^{\prime}\right)=\operatorname{dim} U_{P}^{\theta}$ by (6.3.2).) Since

$$
\mathcal{M}_{\boldsymbol{\lambda}}=\bigcup_{g \in L^{\theta}} g \mathcal{M}_{\left(x^{\prime}, \mathbf{v}^{\prime}\right)} \simeq L^{\theta} \times^{Z_{L^{\theta}}\left(x^{\prime}, \mathbf{v}^{\prime}\right)} \mathcal{M}_{\left(x^{\prime}, \mathbf{v}^{\prime}\right)},
$$

$\mathcal{M}_{\boldsymbol{\lambda}}$ is smooth, irreducible, and open dense in $\overline{\mathcal{M}}_{\boldsymbol{\lambda}}$. Moreover

$$
\operatorname{dim} \mathcal{M}_{\boldsymbol{\lambda}}=\operatorname{dim} \mathcal{O}_{\left(x^{\prime}, \mathbf{v}^{\prime}\right)}+\operatorname{dim} \mathcal{M}_{\left(x^{\prime}, \mathbf{v}^{\prime}\right)}
$$

where $\mathcal{O}_{\left(x^{\prime}, \mathbf{v}^{\prime}\right)}$ is the $L^{\theta}$-orbit of $\left(x^{\prime}, \mathbf{v}^{\prime}\right) \in L_{\text {uni }}^{\iota \theta} \times\left(\prod_{i=1}^{r-2} \bar{M}_{p_{i}} \times \bar{M}_{p_{r-1}}^{\prime}\right)$. We have $\operatorname{dim} \mathcal{O}_{\left(x^{\prime}, \mathbf{v}^{\prime}\right)}=\sum_{i=1}^{r-1} \operatorname{dim} \mathcal{O}_{i}^{\prime}$, where $\mathcal{O}_{i}^{\prime}$ is the $G L\left(\bar{M}_{p_{i}}\right)$-orbit of $\left(x_{i}, \bar{v}_{i}\right)$ for $i=$ $1, \ldots, r-2$, and $\mathcal{O}_{r-1}^{\prime}$ is the $G L\left(\bar{M}_{p_{r-1}}^{\prime}\right)^{\theta}$-orbit of $\left(x_{r-1}, \bar{v}_{r-1}\right)$. Hence by [AH, Prop. 
2.8] and [SS1, Lemma 2.3],

$$
\operatorname{dim} \mathcal{O}_{i}^{\prime}= \begin{cases}m_{i}^{2}-2 n\left(\lambda^{(i)}\right) & \text { for } 1 \leq i \leq r-2 \\ 2 m_{r-1}^{\prime 2}-2 m_{r-1}^{\prime}-4 n\left(\lambda^{(r-1)}+\lambda^{(r)}\right)+2\left|\lambda^{(r-1)}\right| & \text { for } i=r-1\end{cases}
$$

where $m_{r-1}^{\prime}=m_{r-1}+m_{r}$. This implies that

$$
\begin{aligned}
\operatorname{dim} \mathcal{O}_{\left(x^{\prime}, \mathbf{v}^{\prime}\right)}=\sum_{i=1}^{r-2} m_{i}^{2} & +2{m^{\prime}}_{r-1}^{2}-2 m_{r-1}^{\prime} \\
& -2 n(\boldsymbol{\lambda})-2 n\left(\lambda^{(r-1)}+\lambda^{(r)}\right)+2\left|\lambda^{(r-1)}\right| .
\end{aligned}
$$

We have the following lemma.

Lemma 6.6. $\widetilde{\mathcal{F}}_{\boldsymbol{\lambda}}$ is an irreducible variety with

$$
\operatorname{dim} \widetilde{\mathcal{F}}_{\boldsymbol{\lambda}}=2 n^{2}-2 n-2 n(\boldsymbol{\lambda})-2 n\left(\lambda^{(r-1)}+\lambda^{(r)}\right)+\sum_{i=1}^{r-1}(r-i+1)\left|\lambda^{(i)}\right| .
$$

$\widetilde{\mathcal{F}}_{\boldsymbol{\lambda}}^{0}$ is a smooth and open dense subvareity of $\widetilde{\mathcal{F}}_{\boldsymbol{\lambda}}$.

Proof. Since $\widetilde{\mathcal{F}}_{\boldsymbol{\lambda}}=H \times{ }^{P^{\theta}} \overline{\mathcal{M}}_{\boldsymbol{\lambda}}$, and $\overline{\mathcal{M}}_{\boldsymbol{\lambda}}$ is irreducible, $\widetilde{\mathcal{F}}_{\boldsymbol{\lambda}}$ is irreducible. Since $\widetilde{\mathcal{F}}_{\boldsymbol{\lambda}}^{0}=H \times{ }^{P^{\theta}} \mathcal{M}_{\boldsymbol{\lambda}}$, and $\mathcal{M}_{\boldsymbol{\lambda}}$ is smooth and open dense in $\overline{\mathcal{M}}_{\boldsymbol{\lambda}}, \widetilde{\mathcal{F}}_{\boldsymbol{\lambda}}^{0}$ is smooth and open dense in $\widetilde{\mathcal{F}}_{\boldsymbol{\lambda}}$. We have $\operatorname{dim} \widetilde{\mathcal{F}}_{\boldsymbol{\lambda}}=\operatorname{dim} H / P^{\theta}+\operatorname{dim} \mathcal{M}_{\boldsymbol{\lambda}}=\operatorname{dim} U_{P}^{\theta}+\operatorname{dim} \mathcal{M}_{\boldsymbol{\lambda}}$. Here

$$
\begin{aligned}
2 \operatorname{dim} U_{P}^{\theta} & =\operatorname{dim} H-\operatorname{dim} L^{\theta} \\
& =\left(2 n^{2}+n\right)-\left(\sum_{i=1}^{r-2} m_{i}^{2}+2{m^{\prime}}_{r-1}^{2}+m_{r-1}^{\prime}\right) .
\end{aligned}
$$

Then by $(6.5 .1) \sim(6.5 .3)$, we see that

$$
\begin{aligned}
\operatorname{dim} \widetilde{\mathcal{F}}_{\boldsymbol{\lambda}}=2 n^{2}+n-3 m_{r-1}^{\prime}- & 2 n(\boldsymbol{\lambda})-2 n\left(\lambda^{(r-1)}+\lambda^{(r)}\right) \\
+ & \sum_{i=1}^{r-3}(r-i-2) m_{i}+2\left|\lambda^{(r-1)}\right| .
\end{aligned}
$$

The lemma follows from this if we note that $m_{i}=\left|\lambda^{(i)}\right|$ for $i=1, \ldots, r$ and $m_{r-1}^{\prime}=$ $m_{r-1}+m_{r}$.

Proposition 6.7. Assume that $\boldsymbol{\lambda} \in \mathcal{P}_{n, r}$.

(i) $\operatorname{Im} \pi_{\boldsymbol{\lambda}}=\bar{X}_{\boldsymbol{\lambda}}$.

(ii) $\pi_{\boldsymbol{\lambda}}^{0}: \widetilde{\mathcal{F}}_{\boldsymbol{\lambda}}^{0} \rightarrow X_{\boldsymbol{\lambda}}$ gives an isomorphism $\widetilde{\mathcal{F}}_{\boldsymbol{\lambda}}^{0} \underset{\rightarrow}{\rightarrow} X_{\boldsymbol{\lambda}}$. 
(iii) $X_{\boldsymbol{\lambda}}$ is a smooth, irreducible, and

$$
\operatorname{dim} X_{\boldsymbol{\lambda}}=2 n^{2}-2 n-2 n(\boldsymbol{\lambda})-2 n\left(\lambda^{(r-1)}+\lambda^{(r)}\right)+\sum_{i=1}^{r-1}(r-i+1)\left|\lambda^{(i)}\right|
$$

(iv) $X_{\boldsymbol{\lambda}}$ is a locally closed subvariety of $\mathcal{X}_{\text {uni }}$.

Proof. $\pi_{\boldsymbol{\lambda}}^{0}: \widetilde{\mathcal{F}}_{\boldsymbol{\lambda}}^{0} \rightarrow X_{\boldsymbol{\lambda}}$ is $H$-equivariant, and surjective. Take $(x, \mathbf{v}) \in \mathcal{M}_{\boldsymbol{\lambda}}$ and put $z=\left(x, v_{r-1}\right), z^{\prime}=\pi_{P}(z)$. Let $\mathcal{O}^{\prime}$ be the $L^{\theta}$-orbit of $z^{\prime}$. Then

$$
\left(\pi_{\boldsymbol{\lambda}}^{0}\right)^{-1}(x, \mathbf{v}) \simeq\left\{g P^{\theta} \in H / P^{\theta} \mid g^{-1}(x, \mathbf{v}) \in \mathcal{M}_{\boldsymbol{\lambda}}\right\} \subset \mathcal{P}_{z, \mathcal{O}^{\prime}}
$$

where $\mathcal{P}_{z, \mathcal{O}^{\prime}}$ is as in 6.2. By Proposition 6.3 (i), $\mathcal{P}_{z, \mathcal{O}^{\prime}}=\left\{P^{\theta}\right\}$. By the definition of $\widetilde{\mathcal{F}}_{\boldsymbol{\lambda}},\left(\pi_{\boldsymbol{\lambda}}^{0}\right)^{-1}(x, \mathbf{v})$ contains $P^{\theta}$. Hence we see that $\left(\pi_{\lambda}^{0}\right)^{-1}(x, \mathbf{v})=\left\{P^{\theta}\right\}$. It follows that $\pi_{\lambda}^{0}$ is a bijective morphism. The correspondence $g(x, \mathbf{v}) \mapsto g P^{\theta}$ gives a well-defined morphism from the $H$-orbit of $(x, \mathbf{v}) \in \mathcal{M}_{\boldsymbol{\lambda}}$ to $H / P^{\theta}$. This induces a morphism from $X_{\boldsymbol{\lambda}}$ to $\widetilde{\mathcal{F}}_{\boldsymbol{\lambda}}^{0}$. It is easy to check that this gives an inverse of $\pi_{\boldsymbol{\lambda}}^{0}$. Hence $\widetilde{\mathcal{F}}_{\boldsymbol{\lambda}}^{0} \simeq X_{\boldsymbol{\lambda}}$ and (ii) holds. (iii) is immediate from (ii) and Lemma 6.6. By (ii) $\operatorname{Im} \pi_{\boldsymbol{\lambda}}$ contains $X_{\boldsymbol{\lambda}}$. Since $\pi_{\boldsymbol{\lambda}}$ is proper, $\operatorname{Im} \pi_{\boldsymbol{\lambda}}$ is closed, hence $\operatorname{Im} \pi_{\boldsymbol{\lambda}}$ contains $\bar{X}_{\boldsymbol{\lambda}}$. We have $\operatorname{dim} \widetilde{\mathcal{F}}_{\boldsymbol{\lambda}}=\operatorname{dim} X_{\boldsymbol{\lambda}}$ by (iii), and $\operatorname{Im} \pi_{\boldsymbol{\lambda}}, X_{\boldsymbol{\lambda}}$ are irreducible. Thus (i) holds. Here $\widetilde{\mathcal{F}}_{\boldsymbol{\lambda}} \backslash \widetilde{\mathcal{F}}_{\boldsymbol{\lambda}}^{0}$ is a closed subset of $\widetilde{\mathcal{F}}_{\boldsymbol{\lambda}}$, and its image by $\pi_{\boldsymbol{\lambda}}$ coincides with $\bar{X}_{\boldsymbol{\lambda}} \backslash X_{\boldsymbol{\lambda}}$. Hence $\bar{X}_{\boldsymbol{\lambda}} \backslash X_{\boldsymbol{\lambda}}$ is closed. This implies that $X_{\boldsymbol{\lambda}}$ is open dense in $\bar{X}_{\boldsymbol{\lambda}}$, and so (iv) holds. The proposition is proved.

6.8 Assume that $\mathbf{m} \in \mathcal{Q}_{n, r}^{0}$. We define a set $\widetilde{\mathcal{P}}(\mathbf{m})$ by

$$
\widetilde{\mathcal{P}}(\mathbf{m})=\coprod_{0 \leq k \leq m_{r-1}} \mathcal{P}(\mathbf{m}(k))
$$

(see 1.6 for the definition of $\mathbf{m}(k))$. For $\mathbf{m} \in \mathcal{Q}_{n, r}$, let $\boldsymbol{\lambda}(\mathbf{m}) \in \mathcal{P}_{n, r}$ as in 5.8. Let $\bar{X}_{\boldsymbol{\lambda}}$ be the closure of $X_{\boldsymbol{\lambda}}$ in $\mathcal{X}_{\text {uni }}$. As an analogue of Proposition 5.9, we prove the following.

Proposition 6.9. Assume that $\mathbf{m} \in \mathcal{Q}_{n, r}^{0}$.

(i) $\operatorname{dim} \widetilde{\mathcal{X}}_{\mathbf{m}, \text { uni }}=\operatorname{dim} \mathcal{X}_{\mathbf{m}, \text { uni }}$.

(ii) $\mathcal{X}_{\mathbf{m}, \text { uni }}=\bar{X}_{\boldsymbol{\lambda}(\mathbf{m})}$.

(iii) Assume that $\boldsymbol{\mu} \in \widetilde{\mathcal{P}}(\mathbf{m})$. Then $X_{\boldsymbol{\mu}} \subset \mathcal{X}_{\mathbf{m} \text {,uni }}$.

Proof. We consider the surjective map $\pi_{1}^{(\mathbf{m})}: \widetilde{\mathcal{X}}_{\mathbf{m} \text {,uni }} \rightarrow \mathcal{X}_{\mathbf{m} \text {,uni }}$. Take $z=(x, \mathbf{v}) \in$ $\mathcal{M}_{\boldsymbol{\mu}}$ for $\boldsymbol{\mu} \in \mathcal{P}(\mathbf{m}(k))$. Then it follows from the construction of $\mathcal{M}_{\boldsymbol{\mu}}$ that $\left(z, B^{\theta}\right) \in$ $\widetilde{X}_{\mathbf{m} \text {,uni }}$. Hence $z \in X_{\mathbf{m} \text {,uni }}$. Since $X_{\mathbf{m} \text { uni }}$ is $H$-stable, we see that $X_{\boldsymbol{\mu}} \subset \mathcal{X}_{\mathbf{m} \text {,uni. }}$. This proves (iii). Put $\boldsymbol{\mu}=\boldsymbol{\lambda}(\mathbf{m})$. By (iii) we have $X_{\boldsymbol{\mu}} \subset \mathcal{X}_{\mathbf{m} \text {,uni }}$. By (6.1.1),

$$
\operatorname{dim} \widetilde{\mathcal{X}}_{\mathbf{m} \text { uni }}=2 n^{2}-n+\sum_{i=1}^{r-1}(r-i) m_{i}
$$


On the other hand, by Proposition 6.7, we have

$$
\begin{aligned}
\operatorname{dim} X_{\boldsymbol{\mu}} & =2 n^{2}-2 n+\sum_{i=1}^{r-1}(r-i+1) m_{i} \\
& =2 n^{2}-n+\sum_{i=1}^{r-1}(r-i) m_{i}
\end{aligned}
$$

(Note that $\sum_{i=1}^{r-1} m_{i}=n$ since $m_{r}=0$.) Since $\bar{X}_{\boldsymbol{\mu}}$ is a closed, irreducible subset of $\mathcal{X}_{\mathbf{m} \text { uni }}$ with $\operatorname{dim} \bar{X}_{\boldsymbol{\lambda}}=\operatorname{dim} \widetilde{\mathcal{X}}_{\mathbf{m} \text { uni }}$, we conclude that $\operatorname{dim} \widetilde{\mathcal{X}}_{\mathbf{m} \text { uni }}=\operatorname{dim} \mathcal{X}_{\mathbf{m} \text {,uni }}$ and $\bar{X}_{\boldsymbol{\mu}}=\mathcal{X}_{\mathrm{m}, \text { uni }}$. This proves (i) and (ii).

6.10. For a fixed $\mathbf{m} \in \mathcal{Q}_{n, r}$, let $P$ be as in 6.2. For $\boldsymbol{\lambda} \in \mathcal{P}(\mathbf{m})$, we consider the $L^{\theta}$-orbit $\mathcal{O}^{\prime}$ and $H$-orbit $\mathcal{O}$ as in 6.4. Since $\mathcal{O}^{\prime}$ is determined by $\boldsymbol{\lambda}$, we denote it by $\mathcal{O}^{\prime}=\mathcal{O}_{\lambda}^{\prime}$. We shall determine the type of $\mathcal{O}$. For a partition $\nu=(\underbrace{a, \ldots, a}_{k \text {-times }})=\left(a^{k}\right) \in$ $\mathcal{P}_{a k}$ of rectangular type, we define a double partition $[\nu] \in \mathcal{P}_{a k, 2}$ by $[\nu]=\left(a^{k^{\prime}}, a^{k^{\prime \prime}}\right)$, where $k^{\prime}=[k+1 / 2], k^{\prime \prime}=k-k^{\prime}$. In general, a partition $\nu$ can be decomposed uniquely as a sum of partitions of rectangular type

$$
\nu=\left(a_{1}\right)^{k_{1}}+\left(a_{2}\right)^{k_{2}}+\cdots+\left(a_{\ell}\right)^{k_{\ell}}
$$

where we write the dual partition $\lambda^{*}$ as $\lambda^{*}=\left(\left(k_{1}\right)^{a_{1}},\left(k_{2}\right)^{a_{2}}, \ldots,\left(k_{\ell}\right)^{a_{\ell}}\right)$ for $k_{1}>k_{2}>$ $\cdots>k_{\ell}>0$. We define $[\nu] \in \mathcal{P}_{|\nu|, 2}$ by

$$
[\nu]=\left[\left(a_{1}\right)^{k_{1}}\right]+\left[\left(a_{2}\right)^{k_{2}}\right]+\cdots+\left[\left(a_{\ell}\right)^{k_{\ell}}\right]
$$

For a given $\boldsymbol{\lambda} \in \mathcal{P}_{n, r}$, we define $[\boldsymbol{\lambda}] \in \mathcal{P}_{n, 2}$ by

$$
[\boldsymbol{\lambda}]=\left[\lambda^{(1)}\right]+\left[\lambda^{(2)}\right]+\cdots+\left[\lambda^{(r-2)}\right]+\left(\lambda^{(r-1)}, \lambda^{(r)}\right) .
$$

We have the following lemma.

Lemma 6.11. Let $\mathcal{O}$ be a unique $H$-orbit in $G^{\iota \theta} \times V$ such that $\mathcal{O} \cap \pi_{P}^{-1}\left(\mathcal{O}_{\lambda}^{\prime}\right)$ is open dense in $\pi_{P}^{-1}\left(\mathcal{O}_{\lambda}^{\prime}\right)$. Then $\mathcal{O}=\mathcal{O}_{[\lambda]}$.

Proof. We show that $\mathcal{O}_{[\boldsymbol{\lambda}]}$ satisifes the required condition. Take $z \in \mathcal{O}_{[\boldsymbol{\lambda}]}$, and $z^{\prime} \in \mathcal{O}_{\lambda}^{\prime}$. First we show that

$$
\operatorname{dim} Z_{H}(z)=\operatorname{dim} Z_{L^{\theta}}\left(z^{\prime}\right)
$$

In fact, $\operatorname{dim} \mathcal{O}_{\lambda}^{\prime}=\sum_{i=1}^{r-2} \operatorname{dim} \mathcal{O}_{\lambda^{(i)}}^{\prime}+\operatorname{dim} \mathcal{O}_{0}^{\prime}$, where $\mathcal{O}_{\lambda^{(i)}}^{\prime}$ (resp. $\left.\mathcal{O}_{0}^{\prime}\right)$ is the $G L\left(\bar{M}_{p_{i}}\right)$ orbit of type $\lambda^{(i)}$ (resp. $G L\left(\bar{M}_{p_{r-1}}^{\prime}\right)^{\theta}$-orbit of type $\left.\left(\lambda^{(r-1)}, \lambda^{(r)}\right)\right)$ corresponding to 
$\mathcal{O}_{\lambda}^{\prime}$. We have

$$
\begin{aligned}
\operatorname{dim} \mathcal{O}_{\lambda^{(i)}}^{\prime} & =m_{i}^{2}-m_{i}-2 n\left(\lambda^{(i)}\right) \text { for } 1 \leq i \leq r-2 \\
\operatorname{dim} \mathcal{O}_{0}^{\prime} & =2 m_{r-1}^{\prime 2}-2 m_{r-1}^{\prime}-4 n\left(\lambda^{(r-1)}+\lambda^{(r)}\right)+2\left|\lambda^{(r-1)}\right| .
\end{aligned}
$$

(The first formula is well-known, see, e.g. [AH, Prop. $2.8(4)]$. The second one is by [SS1, Lemma 2.3]. ) Hence

$$
\operatorname{dim} Z_{L^{\theta}}\left(z^{\prime}\right)=\sum_{i=1}^{r-2}\left(m_{i}+2 n\left(\lambda^{(i)}\right)\right)+3 m_{r-1}^{\prime}+4 n\left(\lambda^{(r-1)}+\lambda^{(r)}\right)-2\left|\lambda^{(r-1)}\right| .
$$

On the other hand, if we write $[\boldsymbol{\lambda}]=\left(\nu^{\prime}, \nu^{\prime \prime}\right), \operatorname{dim} \mathcal{O}_{[\boldsymbol{\lambda}]}$ is given by a similar formula as $\operatorname{dim} \mathcal{O}_{0}^{\prime}$. Hence we have

$$
\operatorname{dim} Z_{H}(z)=3 n+4 n([\boldsymbol{\lambda}])-2 n\left(\nu^{\prime}\right)
$$

Here, for $i=1, \ldots, r-2$, we show the formula

$$
m_{i}+2 n\left(\lambda^{(i)}\right)=3 m_{i}+4 n\left(\left[\lambda^{(i)}\right]\right)-2\left|\nu_{(i)}{ }^{\prime}\right|,
$$

where we write $\left[\lambda^{(i)}\right]$ as $\left(\nu_{(i)}{ }^{\prime}, \nu_{(i)}{ }^{\prime \prime}\right)$. By the additivitiy of $n$-function together with (6.10.1) and (6.10.2), it is enough to verify (6.11.4) in the case where $\lambda^{(i)}$ is of rectangular type. Assume that $\lambda^{(i)}=\left(a^{2 k-1}\right)$. In this case, $m_{i}=a(2 k-1),\left|\nu_{(i)}^{\prime}\right|=$ $a k, n\left(\lambda^{(i)}\right)=a(k-1)(2 k-1)$ and $n\left(\left[\lambda^{(i)}\right]\right)=a(k-1)^{2}$. Thus (6.11.4) holds. The case where $\lambda^{(i)}=\left(a^{2 k}\right)$ is dealt similarly. Now by substituting (6.11.4) into the formula in (6.11.2), and by comparing it with (6.11.3), we obtain (6.11.1).

In order to show the lemma, it is enough to see that $\mathcal{O}_{[\boldsymbol{\lambda}]} \cap \pi_{P}^{-1}\left(\mathcal{O}_{\lambda}^{\prime}\right) \neq \emptyset$ by Proposition 6.3 (iii). First we show

(6.11.5) Assume that $r=2$ and $m_{1}=n, m_{2}=0$. Then $\mathcal{O}_{[\boldsymbol{\lambda}]} \cap \pi_{P}^{-1}\left(\mathcal{O}_{\boldsymbol{\lambda}}^{\prime}\right) \neq \emptyset$.

In this case $\boldsymbol{\lambda}=\left(\lambda^{(1)}, \emptyset\right),[\boldsymbol{\lambda}]=\left[\lambda^{(1)}\right]$. We consider the variety $G_{\text {uni }} \times V$, and we denote by $\mathbb{O}_{\boldsymbol{\xi}}$ the $G$-orbit in $G_{\text {uni }} \times V$ corresponding to $\boldsymbol{\xi} \in \mathcal{P}_{2 n, 2}$. The map $\pi_{P}$ is naturally extended to a map $\tilde{\pi}_{P}: P_{\text {uni }} \times V \rightarrow L_{\text {uni }} \times V_{L}$. Then by [AH, Cor. 3.4] (see Cor. 5.17 in the case where $r=2$ ), we have

(6.11.6) $\tilde{\pi}_{P}^{-1}\left(\mathcal{O}_{\lambda}^{\prime}\right) \cap \mathbb{O}_{\boldsymbol{\xi}} \neq \emptyset$ if and only if $\boldsymbol{\xi} \leq\left(\lambda^{(1)}, \lambda^{(1)}\right)$ and $\boldsymbol{\xi} \not \boldsymbol{\mu}$ for any $\boldsymbol{\mu}=\left(\mu^{\prime}, \mu^{\prime \prime}\right) \neq\left(\lambda^{(1)}, \lambda^{(1)}\right)$ such that $\mu^{\prime} \leq \lambda^{(1)}, \mu^{\prime \prime} \leq \lambda^{(1)}$.

Moreover, by [AH, Th. 6.1], $\mathbb{O}_{\boldsymbol{\xi}} \cap\left(G_{\text {uni }}^{\iota \theta} \times V\right) \neq \emptyset$ only when $\boldsymbol{\xi}$ is of the form $\boldsymbol{\xi}=\left(\xi^{\prime} \cup \xi^{\prime}, \xi^{\prime \prime} \cup \xi^{\prime \prime}\right)$, and in that case $\mathbb{O}_{\boldsymbol{\xi}} \cap\left(G_{\text {uni }}^{\iota \theta} \times V\right)=\mathcal{O}_{\left(\xi^{\prime}, \xi^{\prime \prime}\right)}$. Write $[\boldsymbol{\lambda}]=\left(\nu^{\prime}, \nu^{\prime \prime}\right)$, and consider $\boldsymbol{\xi}=\left(\nu^{\prime} \cup \nu^{\prime}, \nu^{\prime \prime} \cup \nu^{\prime \prime}\right)$. We show that $\boldsymbol{\xi}$ satisfies the condition in (6.11.6). By the decompostion (6.10.2), we may assume that $\lambda^{(1)}$ is of rectangular type. So we assume that $\lambda^{(1)}=\left(a^{2 k-1}\right)$. In that case, $\left(\lambda^{(1)}, \lambda^{(1)}\right)=\left(\left(a^{2 k-1}\right),\left(a^{2 k-1}\right)\right)$ and $\boldsymbol{\xi}=\left(\left(a^{2 k}\right),\left(a^{2 k-2}\right)\right)$. The condition for (6.11.6) is easily verified. The case where $\lambda^{(1)}=\left(a^{2 k}\right)$ is trivial since $\boldsymbol{\xi}=\left(\lambda^{(1)}, \lambda^{(1)}\right)$. Thus (6.11.5) holds. 
Next we consider the general case. Let $V=V_{1} \oplus \cdots \oplus V_{r-1}$ be the decomposition of $V$ into subspaces, where $V_{i}$ is a symplectic subspace spanned by $\left\{e_{i}, f_{i} \mid p_{i-1}+\right.$ $\left.1 \leq i \leq p_{i}\right\}$ for $i=1, \ldots, r-2$, and $V_{r-1}$ is a symplectic subspace sppaned by $\left\{e_{i}, f_{i} \mid p_{r-2}+1 \leq i \leq n\right\}$. We consider a $\theta$-stable subgroup $\widetilde{L}$ of $G$ containing $L$ such that $\widetilde{L} \simeq G_{1} \times \cdots \times G_{r-1}$ with $G_{i}=G L\left(V_{i}\right)$. Hence $\widetilde{L}^{\theta} \simeq G_{1}^{\theta} \times \cdots \times G_{r-1}^{\theta}$ with $G_{i}^{\theta}=S p\left(V_{i}\right)$. For $i=1, \ldots, r-2$, let $P_{i}$ be a $\theta$-stable parabolic subgroup of $G_{i}$ such that its $\theta$-stable Levi subgroup $L_{i}$ is isomorphic to $G L_{m_{i}} \times G L_{m_{i}}$. Let $\mathcal{O}_{\lambda^{(i)}}^{\prime}$ be the $G L_{m_{i}}$-orbit in $\left(G L_{m_{i}}\right)_{\text {uni }}$ as before. We regard it as a $L_{i}^{\theta}$-orbit in $\left(G_{i}\right)_{\text {uni }}^{\iota \theta}$. Let $\mathcal{O}_{\left[\lambda^{(i)}\right]}$ be the $G_{i}^{\theta}$-orbit in $\left(G_{i}\right)_{\text {uni }}^{\iota \theta} \times V_{i}$ corresponding to $\left[\lambda^{(i)}\right]$. Then by $(6.11 .5)$, one can find $z_{i} \in \mathcal{O}_{\left[\lambda^{(i)}\right]} \cap \pi_{P_{i}}^{-1}\left(\mathcal{O}_{\lambda^{(i)}}^{\prime}\right)$. Let $\mathcal{O}_{0}^{\prime}$ be the $G_{r-1}^{\theta}$-orbit in $\left(G_{r-1}\right)_{\text {uni }}^{\iota \theta}$ corresponding to $\left(\lambda^{(r-1)}, \lambda^{(r)}\right)$. We choose $z_{r-1} \in \mathcal{O}_{0}^{\prime}$. Since $\prod_{i=1}^{r-2} \pi_{P_{i}}^{-1}\left(\mathcal{O}_{\lambda^{(i)}}^{\prime}\right) \times \mathcal{O}_{r-1}^{\prime}$ is regarded as a closed subvariety of $\pi_{P}^{-1}\left(\mathcal{O}_{\lambda}^{\prime}\right), z=\left(z_{1}, \ldots, z_{r-1}\right)$ gives an element in $\pi_{P}^{-1}\left(\mathcal{O}_{\lambda}^{\prime}\right)$. $z$ is contained in $\widetilde{L}^{\iota \theta} \times V$, and actually $z \in \mathcal{O}_{[\boldsymbol{\lambda}]}$. Thus $\mathcal{O}_{[\boldsymbol{\lambda}]} \cap \pi_{P}^{-1}\left(\mathcal{O}_{\lambda}^{\prime}\right) \neq \emptyset$. The lemma is proved.

Corollary 6.12. For each $\boldsymbol{\lambda} \in \mathcal{P}_{n, r}$, the map $(x, \mathbf{v}) \mapsto\left(\left(x, v_{r-1}\right),\left(v_{1}, \ldots, v_{r-2}\right)\right)$ gives an embedding $X_{\boldsymbol{\lambda}} \subset \mathcal{O}_{[\boldsymbol{\lambda}]} \times V^{r-2}$.

Remark 6.13. Proposition 6.9 shows that $\bigcup_{\boldsymbol{\lambda} \in \mathcal{P}_{n, r}} X_{\boldsymbol{\lambda}}$ covers a dense subset of $\mathcal{X}_{\text {uni }}$. However it does not coincide with $\mathcal{X}_{\text {uni }}$ in general. In the case where $n=2, r=3$, one can show by a direct computation that $X_{\boldsymbol{\lambda}}$ are mutually disjoint for $\boldsymbol{\lambda} \in \mathcal{P}_{n, r}$. It is not known whether the disjointness property holds for $X_{\boldsymbol{\lambda}}$ in general.

6.14. The unipotent variety of enhanced type considered in Section 5 can be interpreted as a closed subvariety of the variety defined in 1.2. So we follow the setting in 1.2 , and consider the varieties $\widetilde{\mathcal{X}}_{\mathbf{m}}, \mathcal{X}_{\mathbf{m}}$ as in Section 4 . Then the vareities $\widetilde{\mathcal{X}}_{\mathbf{m} \text {,uni }}, \mathcal{X}_{\mathbf{m} \text {,uni }}$ defined in 5.2 can be identified with closed subvarieties of $\widetilde{\mathcal{X}}_{\mathbf{m}}, \mathcal{X}_{\mathbf{m}}$, defined by similar formulas as in 6.1. We shall reformulate $X_{\boldsymbol{\lambda}}$ defined in Section 5 so that it fits to the discussion in the exotic case.

First we consider an analogue of Propostion 6.3 in the enhanced case. Assume that $r \geq 2$, and take $\mathbf{m} \in \mathcal{Q}_{n, r}$. Let $P$ be a $\theta$-stable parabolic subgroup of $G$ such that $P^{\theta}$ is the stabilizer of the flag $\left(M_{p_{i}}\right)_{1 \leq i \leq r}$ in $V$. Let $L$ be the $\theta$-stble Levi subgroup of $P$ containing $T$. Contrast to 6.2 , we put $V_{L}=V$. We consider the $\operatorname{map} \pi_{P}: P_{\text {uni }}^{\iota \theta} \times V \rightarrow L_{\text {uni }}^{\iota \theta} \times V_{L},(x, v) \mapsto\left(x^{\prime}, v\right)$, where $x^{\prime}=p(x)$ for the natural projection $p: P_{\text {uni }}^{\iota \theta} \rightarrow L_{\text {uni }}^{\iota \theta}$. We define $\mathcal{O}, \mathcal{O}^{\prime}$ and $\mathcal{P}_{z, \mathcal{O}^{\prime}}$ as in 6.2. The following result can be proved word by word following the proof of Proposition 6.3. Note that in the enhanced case, $\operatorname{dim} U_{P}^{\theta}=\operatorname{dim} U_{P}^{\iota \theta}$.

Proposition 6.15. Assume that $\mathcal{X}_{\text {uni }}=G_{\text {uni }}^{\iota \theta} \times V$ is of enhaned type. Then similar statements $(i) \sim(v i)$ as in Proposition 6.3 hold for $\mathcal{X}_{\text {uni }}$.

6.16. Let $P$ be as in 6.14. For $\boldsymbol{\lambda} \in \mathcal{P}(\mathbf{m})$, by imitating the definition of $\mathcal{M}_{\boldsymbol{\lambda}}$ in 6.4, we define a subset $\mathcal{M}_{\boldsymbol{\lambda}}$ of $P_{\text {uni }}^{\iota \theta} \times \prod_{i=1}^{r-1} M_{p_{i}}$ as the set of $(x, \mathbf{v})$ satisfying the following properties; take $(x, \mathbf{v})$ such that $x \in P_{\text {uni }}^{\iota \theta}$ and that $v_{i} \in M_{p_{i}}$ for $i=1, \ldots, r-1$. Put $\bar{M}_{p_{i}}=M_{p_{i}} / M_{p_{i-1}}$, and let $\bar{v}_{i} \in \bar{M}_{p_{i}}$ be the image of $v_{i}$ for each $i$. We assume that $G L\left(\bar{M}_{p_{i}}\right)$-orbit of $\left(\left.x\right|_{M_{p_{i}}}, \bar{v}_{i}\right)$ in $G L\left(\bar{M}_{p_{i}}\right)^{\iota \theta} \times \bar{M}_{p_{i}}$ has type $\left(\lambda^{(i)}, \emptyset\right)$ for each $i$. We further assume that 
(6.16.1) $\mathcal{O} \cap p^{-1}\left(\mathcal{O}^{\prime}\right)$ is open dense in $p^{-1}\left(\mathcal{O}^{\prime}\right)$, where $\mathcal{O}$ is the $H$-orbit of $x$ in $G_{\text {uni }}^{\iota \theta}$ and $\mathcal{O}^{\prime}$ is the $L^{\theta}$-orbit of $x^{\prime}=p(x) \in L_{\text {uni }}^{\iota \theta}$.

We define $X_{\boldsymbol{\lambda}}^{\prime}$ by $X_{\boldsymbol{\lambda}}^{\prime}=\bigcup_{g \in H} g \mathcal{M}_{\boldsymbol{\lambda}} . \mathcal{M}_{\boldsymbol{\lambda}}$ is a $P^{\theta}$-stable subset of $P^{\imath \theta} \times V^{r-1}$. We have a lemma.

Lemma 6.17. $X_{\boldsymbol{\lambda}}^{\prime}$ coincides with $X_{\boldsymbol{\lambda}}$ for each $\boldsymbol{\lambda} \in \mathcal{P}_{n, r}$. In particular, $X_{\boldsymbol{\lambda}}^{\prime}$ 's are mutually disjoint.

Proof. Assume that $\mathcal{O}^{\prime}=\mathcal{O}_{\lambda}^{\prime}$. Then the $H$-orbit $\mathcal{O}$ in (6.16.1) is given by $\mathcal{O}=\mathcal{O}_{\nu}$ with $\nu=\lambda^{(1)}+\cdots+\lambda^{(r)}$. The lemma then follows from Corollary 5.17 (ii).

6.18. As in the exotic case, we defnie a variety $\widetilde{\mathcal{F}}_{\boldsymbol{\lambda}}=H \times^{P^{\theta}} \overline{\mathcal{M}}_{\boldsymbol{\lambda}}$, where $\overline{\mathcal{M}}_{\boldsymbol{\lambda}}$ is the closure of $\mathcal{M}_{\boldsymbol{\lambda}}$ in $P^{\iota \theta} \times V^{r-1}$. Then the map $\pi_{\boldsymbol{\lambda}}^{\prime}: \widetilde{\mathcal{F}}_{\boldsymbol{\lambda}} \rightarrow X_{\boldsymbol{\lambda}}^{\prime}=X_{\boldsymbol{\lambda}}$ is defined as in 6.4. (Here we use the notation $\pi_{\boldsymbol{\lambda}}^{\prime}$ to distinguish it from the map $\pi_{\boldsymbol{\lambda}}: \widetilde{X}_{\boldsymbol{\lambda}} \rightarrow X_{\boldsymbol{\lambda}}$ in 5.5.) We also define a subset $\widetilde{\mathcal{F}}_{\boldsymbol{\lambda}}^{0}$ of $\widetilde{\mathcal{F}}_{\boldsymbol{\lambda}}$ by $\widetilde{\mathcal{F}}_{\boldsymbol{\lambda}}^{0}=H \times{ }^{P^{\theta}} \mathcal{M}_{\boldsymbol{\lambda}}$, and let $\pi_{\boldsymbol{\lambda}}^{\prime 0}$ be the restriciton of $\pi_{\lambda}^{\prime}$ on $\widetilde{\mathcal{F}}_{\lambda}^{0}$. The following result can be proved by a similar way as Proposition 6.7 .

Proposition 6.19. Assume that $\mathcal{X}$ is of enhanced type. For each $\boldsymbol{\lambda} \in \mathcal{P}_{n, r}$,

(i) $\operatorname{Im} \pi_{\lambda}^{\prime}=\bar{X}_{\lambda}$.

(ii) $\pi_{\lambda}^{\prime 0}: \widetilde{\mathcal{F}}_{\boldsymbol{\lambda}}^{0} \rightarrow X_{\boldsymbol{\lambda}}$ gives an isomorphism $\widetilde{\mathcal{F}}_{\boldsymbol{\lambda}}^{0} \underset{\rightarrow}{\rightarrow} X_{\boldsymbol{\lambda}}$.

6.20. In the remainder of this section, we assume that $\mathcal{X}$ is of extoic type or of enhanced type. We follow the formulation in Section 1. Put $\mathcal{B}=H / B^{\theta}$. For each $z=(x, \mathbf{v}) \in \mathcal{X}_{\mathbf{m}, \text { uni }}$, put

$$
\begin{gathered}
\mathcal{B}_{z}=\left\{g B^{\theta} \in \mathcal{B} \mid g^{-1} x g \in U^{\iota \theta}, g^{-1} \mathbf{v} \in M_{n}^{r-1}\right\} \\
\mathcal{B}_{z}^{(\mathbf{m})}=\left\{g B^{\theta} \in \mathcal{B} \mid g^{-1} x g \in U^{\iota \theta}, g^{-1} \mathbf{v} \in \prod_{i=1}^{r-1} M_{p_{i}}\right\} .
\end{gathered}
$$

In the exotic case, $\mathcal{B}_{z}$ is a closed subvariety of $\mathcal{B}$, which is isomorphic to the fibre $\pi_{1}^{-1}(z)$, and is called the Springer fibre of $z . \mathcal{B}_{z}^{(\mathbf{m})}$ is a closed subvariety of $\mathcal{B}_{z}$ isomorphic to $\left(\pi_{1}^{(\mathbf{m})}\right)^{-1}(z)$, which we call the small Springer fibre. In the enhanced case, we only consider the Springer fibre $\mathcal{B}_{z}^{(\mathbf{m})}$ as alreday defined in 5.18.

We fix $\mathbf{m} \in \mathcal{Q}_{n, r}$. For an integer $d \geq 0$, we define a subset $X(d)$ of $\mathcal{X}_{\mathbf{m} \text {,uni }}$ by

$$
X(d)=\left\{z \in \mathcal{X}_{\mathbf{m}, \text { uni }} \mid \operatorname{dim} \mathcal{B}_{z}^{(\mathbf{m})}=d\right\}
$$


Then $X(d)$ is a locally closed subvariety of $\mathcal{X}_{\mathbf{m} \text {,uni }}$, and $\mathcal{X}_{\mathbf{m} \text {,uni }}=\coprod_{d \geq 0} X(d)$. We consider the Steinberg varieties $\mathcal{Z}^{(\mathbf{m})}$ and $\mathcal{Z}_{1}^{(\mathbf{m})}$ as follows;

$$
\begin{aligned}
\mathcal{Z}^{(\mathbf{m})}=\left\{\left(z, g B^{\theta}, g^{\prime} B^{\theta}\right) \in \mathcal{X} \times \mathcal{B} \times \mathcal{B}\right. \\
\left.\mid\left(z, g B^{\theta}\right) \in \widetilde{\mathcal{X}}_{\mathbf{m}},\left(z, g^{\prime} B^{\theta}\right) \in \widetilde{\mathcal{X}}_{\mathbf{m}}\right\} \\
\mathcal{Z}_{1}^{(\mathbf{m})}=\left\{\left(z, g B^{\theta}, g^{\prime} B^{\theta}\right) \in \mathcal{X}_{\text {uni }} \times \mathcal{B} \times \mathcal{B}\right. \\
\left.\mid\left(z, g B^{\theta}\right) \in \widetilde{\mathcal{X}}_{\mathbf{m}, \text { uni }},\left(z, g^{\prime} B^{\theta}\right) \in \widetilde{\mathcal{X}}_{\mathbf{m}, \text { uni }}\right\} .
\end{aligned}
$$

Recall that $W=N_{H}\left(T^{\theta}\right) / T^{\theta}$ is the Weyl group $W_{n}$ of type $C_{n}$ (resp. $S_{n}$ ) in the exotic case (resp. the enhanced case). In the exotic case, we assume that $\mathbf{m} \in \mathcal{Q}_{n, r}^{0}$, while in the enhanced case, we consider an arbitrary $\mathbf{m} \in \mathcal{Q}_{n, r}$. We define a subgroup $\mathcal{W}_{\mathbf{m}}^{\natural}$ of $N_{H}\left(T^{\theta}\right) / T^{\theta}$ by by

$$
\mathcal{W}_{\mathbf{m}}^{\natural}= \begin{cases}S_{m_{1}} \times \cdots \times S_{m-2} \times W_{m-1} & \text { (exotic case) } \\ S_{m_{1}} \times \cdots \times S_{m_{r}} & \text { (enhanced case) } .\end{cases}
$$

We show the following lemma.

Lemma 6.21. Under the notation of 6.20,

(i) $\operatorname{dim} \mathcal{Z}_{1}^{(\mathbf{m})}=\operatorname{dim} \mathcal{X}_{\mathbf{m} \text {,uni }}$. The set of irreducible components of $\mathcal{Z}_{1}^{(\mathbf{m})}$ with maximal dimension is parametrized by $w \in \mathcal{W}_{\mathbf{m}}^{\natural}$.

(ii) $\operatorname{dim} \mathcal{Z}^{(\mathbf{m})}=\operatorname{dim} \mathcal{Z}_{1}^{(\mathbf{m})}+n$. The set of irreducible components of $\mathcal{Z}^{(\mathbf{m})}$ with maximal dimension is parametrized by $w \in \mathcal{W}_{\mathbf{m}}^{\natural}$.

(iii) Assume that $X(d) \neq \emptyset$. For any $z \in X(d)$ we have

$$
\operatorname{dim} \mathcal{B}_{z}^{(\mathbf{m})} \leq \frac{1}{2}\left(\operatorname{dim} \mathcal{X}_{\mathbf{m}, \text { uni }}-\operatorname{dim} X(d)\right)
$$

In particular, $\pi^{(\mathbf{m})}$ is semismall with respect to the stratification $\mathcal{X}_{\mathbf{m} \text {,uni }}=$ $\coprod_{d} X(d)$.

Proof. Let $p_{1}: \mathcal{Z}_{1}^{(\mathbf{m})} \rightarrow \mathcal{B} \times \mathcal{B}$ be the projection on secod and third factors. For each $w \in W$, let $\mathcal{O}_{w}$ be the $H$-orbit of $\left(B^{\theta}, w B^{\theta}\right)$ in $\mathcal{B} \times \mathcal{B}$. We have $\mathcal{B} \times \mathcal{B}=\coprod_{w \in W} \mathcal{O}_{w}$. Put $Z_{w}=p_{1}^{-1}\left(\mathcal{O}_{w}\right)$. Then $Z_{w}$ is a vector bundle over $\mathcal{O}_{w} \simeq H /\left(B^{\theta} \cap w B^{\theta} w^{-1}\right)$ with fibre isomorphic to

$$
\left(U^{\iota \theta} \cap w U^{\iota \theta} w^{-1}\right) \times \prod_{i=1}^{r-1}\left(M_{p_{i}} \cap w\left(M_{p_{i}}\right)\right) .
$$

First we consider the exotic case. We identify $W$ with a subgroup of $S_{2 n}$ which is the stabilizer of the element $(1, n+1)(2, n+2) \cdots(n, 2 n)$. Let $b_{w}$ be the number of $i$ such that $w^{-1}\left(e_{i}\right) \in M_{n}$, i.e., $w^{-1}(i) \in[1, n]$. Then we have $\operatorname{dim}\left(U^{\iota \theta} \cap w U^{\iota \theta} w^{-1}\right)=$ $\operatorname{dim}\left(U^{\theta} \cap w U^{\theta} w^{-1}\right)-b_{w}$. Also we have $\operatorname{dim}\left(M_{n} \cap w\left(M_{n}\right)\right)=b_{w}$. By our assumption 
$M_{n-1}=M_{n}$, we have

$$
\operatorname{dim} Z_{w}=\operatorname{dim} H-\operatorname{dim} T^{\theta}+\sum_{i=1}^{r-2} \operatorname{dim}\left(M_{p_{i}} \cap w\left(M_{p_{i}}\right)\right)
$$

Here $\operatorname{dim}\left(M_{p_{i}} \cap w\left(M_{p_{i}}\right)\right)=\sharp\left\{j \in\left[1, p_{i}\right] \mid w^{-1}(j) \in\left[1, p_{i}\right]\right\} \leq p_{i}$, and the equality holds if and only if $w$ leaves the set $\left[1, p_{i}\right]$ invariant. It follows that $\operatorname{dim} Z_{w}$ takes the maximal value $2 n^{2}+\sum_{i=1}^{r-2} p_{i}$ if and only if $w \in \mathcal{W}_{\mathbf{m}}^{\natural}$. Since

$$
\sum_{i=1}^{r-2} p_{i}=-n+\sum_{i=1}^{r-1}(r-i) m_{i}
$$

(note that $m_{r}=0$ ), in that case $\operatorname{dim} Z_{w}=\operatorname{dim} \mathcal{X}_{\mathbf{m} \text {,uni }}$ by Proposition 6.9 together with (6.1.1). Since $\left\{\bar{Z}_{w} \mid w \in W\right\}$ gives the set of irreducible components of $\mathcal{Z}_{1}^{(\mathbf{m})}$, (i) follows. In the enhanced case, a similar computation shows that

$$
\operatorname{dim} Z_{w}=\operatorname{dim} H-\operatorname{dim} T^{\theta}+\sum_{i=1}^{r-1} \operatorname{dim}\left(M_{p_{i}} \cap w\left(M_{p_{i}}\right)\right)
$$

for $w \in S_{n}$ (note that in this case $\operatorname{dim}\left(U^{\iota \theta} \cap w U^{\imath \theta} w^{-1}\right)=\operatorname{dim}\left(U^{\theta} \cap w U^{\theta} w^{-1}\right)$ ), and $\operatorname{dim} Z_{w}$ takes the maximal value $n^{2}-n+\sum_{i=1}^{r-1} p_{i}$ if and only if $w \in \mathcal{W}_{\mathbf{m}}^{\natural}$. Since $\sum_{i=1}^{r-1} p_{i}=\sum_{i=1}^{r-1}(r-i) m_{i}$, in this case $\operatorname{dim} Z_{w}=\operatorname{dim} \mathcal{X}_{\mathbf{m} \text {,uni }}$ by Proposition 5.9 (ii). Hence (i) holds also in the enhanced case.

For (ii), we consider $\widetilde{Z}_{w}=p^{-1}\left(\mathcal{O}_{w}\right)$, where $p: \mathcal{Z}^{(\mathbf{m})} \rightarrow \mathcal{B} \times \mathcal{B}$ is the projection on the second and third factors. Then $\widetilde{Z}_{w}$ is a locally trivial fibration over $\mathcal{O}_{w}$ with fibre isomorphic to

$$
T^{\iota \theta} \times\left(U^{\iota \theta} \cap w U^{\iota \theta} w^{-1}\right) \times \prod_{i=1}^{r-1}\left(M_{p_{i}} \cap w\left(M_{p_{i}}\right)\right) .
$$

Hence (ii) is proved by a similar argument as (i).

We show (iii). Let $q_{1}: \mathcal{Z}_{1}^{(\mathbf{m})} \rightarrow \mathcal{X}_{\mathbf{m} \text {,uni }}$ be the projection on the first factor. For each $z \in \mathcal{X}_{\mathbf{m} \text {,uni }}, q_{1}^{-1}(z) \simeq \mathcal{B}_{z}^{(\mathbf{m})} \times \mathcal{B}_{z}^{(\mathbf{m})}$. By $(6.20 .1)$, we have

$$
\operatorname{dim} q_{1}^{-1}(X(d))=\operatorname{dim} X(d)+2 d .
$$

Since $\operatorname{dim} q_{1}^{-1}(X(d)) \leq \operatorname{dim} \mathcal{Z}_{1}^{(\mathbf{m})}=\operatorname{dim} \mathcal{X}_{\mathbf{m}, \text { uni }}$, we see that $2 d \leq \operatorname{dim} \mathcal{X}_{\mathbf{m}, \text { uni }}-$ $\operatorname{dim} X(d)$. This proves (iii). The lemma is proved. 


\section{SPRINGER CORRESPONDENCE}

7.1. In this section, we assume that $\mathcal{X}$ is of exotic type or of enhanced type. We shall prove the Springer correspondence for $\mathcal{X}_{\text {uni }}$. However the method used in [SS1], which is based on a evaluation of the number of irreducible components of Springer fibres (see [SS1, Lemma 2.5]), does not work well for the case where $r \geq 3$. Instead, we apply the method used by Lusztig [Lu] to prove the generalized Springer correspondence for reductive groups, which makes use of the Steinberg map. We follow the notation in Section 1. In the exotic case, let $\omega^{\prime}: G \rightarrow T / S_{2 n}$ be the Steinberg map with respect to $G$. Then we have $\Xi=T^{\iota \theta} / S_{n} \hookrightarrow T / S_{2 n}$, where $S_{n}$ is embedded in $S_{2 n}$ as a subgroup of the centralizer of $(1, n+1)(2, n+2) \cdots(n, 2 n)$. In the enhanced case, let $\omega^{\prime}: G \rightarrow T /\left(S_{n} \times S_{n}\right)$ be the Steinberg map and conisder $\Xi=T^{\iota \theta} / S_{n} \hookrightarrow T /\left(S_{n} \times S_{n}\right)$. In either case, we denote by $\omega$ the map $G^{\iota \theta} \rightarrow \Xi$ induced from $\omega^{\prime}$. Take $\mathbf{m} \in \mathcal{Q}_{n, r}$, and consider the map $\pi^{(\mathbf{m})}: \widetilde{\mathcal{X}}_{\mathbf{m}} \rightarrow \mathcal{X}_{\mathbf{m}}$ as in 1.1. Let $\mathcal{Z}^{(\mathbf{m})}$ be the Steinberg variety with respect to $\pi^{(\mathbf{m})}$ defined in 6.20 . We denote by $\varphi$ the natural map $\mathcal{Z}^{(\mathbf{m})} \rightarrow \mathcal{X}_{\mathbf{m}}$. We define a map $\widetilde{\alpha}: \mathcal{Z}^{(\mathbf{m})} \rightarrow T^{\iota \theta}$ by $\left(x, \mathbf{v}, g B^{\theta}, g^{\prime} B^{\theta}\right) \mapsto p_{T}\left(g^{-1} x g\right)$. Then we have a commutative diagram

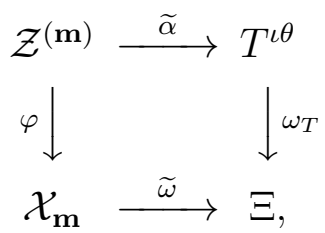

where $\widetilde{\omega}$ is the composite of the projection $\mathcal{X}_{\mathbf{m}} \rightarrow G^{\iota \theta}$ with $\omega$, and $\omega_{T}$ is the restriction of $\omega$ on $T^{\iota \theta}$. Note that $\omega_{T}$ is a finite morphism.

As in 6.20 , we assume that $\mathbf{m} \in \mathcal{Q}_{n, r}^{0}$ in the exotic case, while we consider arbitrary $\mathbf{m} \in \mathcal{Q}_{n, r}$ in the enhanced case. Recall that $d_{\mathbf{m}}^{\prime}=\operatorname{dim} \mathcal{X}_{\mathbf{m} \text {,uni }}$, and put $\sigma=\omega_{T} \circ \widetilde{\alpha}$. We define a constructible sheaf $\mathcal{T}$ on $\Xi$ by

$$
\mathcal{T}=\mathcal{H}^{2 d_{\mathbf{m}}^{\prime}}\left(\sigma_{!} \overline{\mathbf{Q}}_{l}\right)=R^{2 d_{\mathbf{m}}^{\prime}} \sigma_{!} \overline{\mathbf{Q}}_{l}
$$

Recall the definition of perfect sheaves in [Lu, 5.4]. A constructible sheaf $\mathcal{E}$ on an irreducible variety $X$ is said to be perfect if $\mathcal{E}$ coincides with an intersection cohomology complex (reduced to a single sheaf on degeree zero) on $X$, and the support of any nonzero constructible subsheaf of $\mathcal{E}$ is $X$.

The following gives examples of perfect sheaves.

(7.1.2) If $\pi: Y \rightarrow X$ is a finite morphism with $Y$ smooth and if $\mathcal{E}^{\prime}$ is a locally constant sheaf on $Y$, then $\mathcal{E}=\pi_{*} \mathcal{E}^{\prime}$ is a perfect sheaf on $X$.

(7.1.3) If $0 \rightarrow \mathcal{E}_{1} \rightarrow \mathcal{E}_{2} \rightarrow \mathcal{E}_{3} \rightarrow 0$ is an exact sequence of constructible sheaves on $X$, with $\mathcal{E}_{1}, \mathcal{E}_{3}$ perfect, then $\mathcal{E}_{2}$ is perfect.

We show the following lemma.

Lemma 7.2. The sheaf $\mathcal{T}$ is a perfect sheaf on $\Xi$.

Proof. Under the notation in the proof of Lemma 6.20 , we consider $\widetilde{Z}_{w}=p^{-1}\left(\mathcal{O}_{w}\right)$ for each $w \in W$. Let $\sigma_{w}$ be the restriction of $\sigma$ on $\widetilde{Z}_{w}$, and put $\mathcal{T}_{w}=\mathcal{H}^{2 d_{\mathbf{m}}^{\prime}}\left(\left(\sigma_{w}\right) ! \overline{\mathbf{Q}}_{l}\right)$. 
We also put $\alpha_{w}$ the restriction of $\widetilde{\alpha}$ on $\widetilde{Z}_{w}$. Let $\mathcal{W}_{\mathbf{m}}^{\natural}$ be the subgroup of $W$ given in (6.20.2). We show that

$$
\mathcal{T}_{w} \simeq \begin{cases}\left(\omega_{T}\right) ! \overline{\mathbf{Q}}_{l} & \text { if } w \in \mathcal{W}_{\mathbf{m}}^{\natural} \\ 0 & \text { otherwise. }\end{cases}
$$

In fact, since $\widetilde{Z}_{w} \rightarrow \mathcal{O}_{w}$ is a locally trivial fibration with fibre isomorphic to (6.21.1), we see that $\alpha_{w}$ is a locally trivial fibration with fibre isomorphic to

$$
H \times^{\left(B^{\theta} \cap w B^{\theta} w^{-1}\right)}\left(\left(U^{\iota \theta} \cap w U^{\iota \theta} w^{-1}\right) \times \prod_{i=1}^{r-1}\left(M_{p_{i}} \cap w\left(M_{p_{i}}\right)\right)\right) .
$$

By the computation in the proof of Lemma 6.21 , we see that $\operatorname{dim} \alpha_{w}^{-1}(s) \leq d_{\mathbf{m}}^{\prime}$ for any $s \in T^{\llcorner\theta}$, and that the equality holds if and only if $w \in \mathcal{W}_{\mathbf{m}}^{\natural}$. Moreover in that case, each fibre is an irreducible variety. It follows that

$$
R^{2 d_{\mathbf{m}}^{\prime}}\left(\alpha_{w}\right)_{!} \overline{\mathbf{Q}}_{l} \simeq \begin{cases}\overline{\mathbf{Q}}_{l} & \text { if } w \in \mathcal{W}_{\mathbf{m}}^{\natural} \\ 0 & \text { otherwise. }\end{cases}
$$

Since $\omega_{T}$ is a finite morphism, we have $R^{2 d_{\mathbf{m}}^{\prime}}\left(\sigma_{w}\right) ! \overline{\mathbf{Q}}_{l} \simeq R^{0}\left(\omega_{T}\right) ! R^{2 d_{\mathbf{m}}^{\prime}}\left(\alpha_{w}\right) ! \overline{\mathbf{Q}}_{l}$. Thus (7.2.1) follows from (7.2.3).

Since $\omega_{T}$ is a finite morphism, (7.2.1) and (7.1.2) imply that $\mathcal{T}_{w}$ is a perfect sheaf if $w \in \mathcal{W}_{\mathbf{m}}^{\natural}$. By (7.2.2), each fibre $\alpha_{w}^{-1}(s)$ is a vector bundle over $\mathcal{O}_{w}$. In turn, $\mathcal{O}_{w}$ is a vector bundle over $H / B^{\theta}$ with fibre isomorphic to $U^{\theta} \cap w U^{\theta} w^{-1}$. It follows that $H_{c}^{i}\left(\alpha_{w}^{-1}(s), \overline{\mathbf{Q}}_{l}\right)=0$ for odd $i$. This implies that $R^{2 d_{\mathbf{m}}^{\prime}-1}\left(\sigma_{w}\right)_{!} \overline{\mathbf{Q}}_{l}=0$ for $w \in W_{n}$. Now $\mathcal{W}_{\mathbf{m}}^{\natural}$ is a parabolic subgroup of $W$, and the closure relations for $\mathcal{O}_{w}\left(w \in \mathcal{W}_{\mathbf{m}}^{\natural}\right)$ are described by the Bruhat order on $\mathcal{W}_{\mathbf{m}}^{\natural}$. It follows, by a similar argument as in $[\mathrm{Lu}, 5.4]$ by using the property $(7.1 .3)$, we see that $\mathcal{T}$ is a perfect sheaf on $\Xi$. The lemma is proved.

Proposition 7.3. $\mathcal{T} \simeq \bigoplus_{w \in \mathcal{W}_{\mathrm{m}}^{\natural}} \mathcal{T}_{w}$ as sheaves on $\Xi$.

Proof. Put $\Xi_{\text {reg }}=\omega_{T}\left(T_{\text {reg }}^{\iota \theta}\right)$. Then $\Xi_{\text {reg }}$ is an open dense subset of $\Xi$. Since $\mathcal{T}$ and $\bigoplus_{w} \mathcal{T}_{w}$ are perfect sheaves on $\Xi$, it is enough to show that their restrictions on $\Xi_{\text {reg }}$ are isomorphic. Put $\mathcal{Z}_{0}^{(\mathbf{m})}=\sigma^{-1}\left(\Xi_{\text {reg }}\right)$. Then $\mathcal{Z}_{0}^{(\mathbf{m})} \simeq \widetilde{\mathcal{Y}}_{\mathbf{m}} \times_{\mathcal{Y}_{\mathbf{m}}} \widetilde{\mathcal{Y}}_{\mathbf{m}}$. Let $\sigma_{0}$ be the restriction of $\sigma$ on $\mathcal{Z}_{0}^{(\mathbf{m})}$, which is a composite of the natural map $\mathcal{Z}_{0}^{(\mathbf{m})} \rightarrow \mathcal{Y}_{\mathbf{m}}$ with the map $\mathcal{Y}_{\mathbf{m}} \rightarrow \Xi$. The restriction of $\mathcal{T}$ on $\Xi_{\text {reg }}$ is isomorphic to $R^{2 d_{\mathbf{m}}^{\prime}}\left(\sigma_{0}\right)_{!} \overline{\mathbf{Q}}_{l}$. Put

$$
\mathbf{M}^{b}=\prod_{i=1}^{r-1}\left(M_{\left[p_{i-1}+1, p_{i}\right]}+M_{p_{i-1}}\right)
$$

Then $\mathbf{M}^{b}$ coincides with $\mathbf{M}_{\mathbf{I}^{\circ}}$ for $\mathbf{I}^{\circ}=\left(I_{1}^{\circ}, \ldots, I_{r}^{\circ}\right)$ in 1.3 , where $I_{i}^{\circ}=\left[p_{i-1}+1, p_{i}\right]$. Put $\widetilde{\mathcal{Y}}^{b}=\widetilde{\mathcal{Y}}_{\mathbf{I}^{\circ}}$ in the notation in 1.3 (see also 4.1). Under the isomorphism $(1.2 .2$ ), $\widetilde{\mathcal{Y}}^{b}$ is regarded as an open dense subset of $\widetilde{\mathcal{Y}}_{\mathbf{m}}$. Put $\mathcal{Z}^{b}=\widetilde{\mathcal{Y}}^{b} \times \mathcal{Y}_{\mathbf{m}} \widetilde{\mathcal{Y}}^{b}$ and let $\sigma_{b}$ be 
the restriction of $\sigma_{0}$ on $\mathcal{Z}^{b}$. For each $w \in W_{n}$, let $\sigma_{b}^{w}$ be the restriction of $\sigma_{b}$ on $\mathcal{Z}^{b}(w)=\mathcal{Z}^{b} \cap \widetilde{Z}_{w}$. Note that $\mathcal{Z}^{b}(w)=\emptyset$ unless $w \in \mathcal{W}_{\mathbf{m}}^{\natural}$. Now $\mathcal{Z}^{b}$ is an open subset of $\mathcal{Z}_{0}^{(\mathbf{m})}$ and the inclusion map $\mathcal{Z}^{\mathrm{b}} \hookrightarrow \mathcal{Z}_{0}^{(\mathbf{m})}$ induces a morphism $R^{i}\left(\sigma_{b}\right)_{!} \overline{\mathbf{Q}}_{l} \rightarrow R^{i}\left(\sigma_{0}\right) ! \overline{\mathbf{Q}}_{l}$ for each $i$. Similarly, we have a morphism $R^{i}\left(\sigma_{b}^{w}\right)_{!} \overline{\mathbf{Q}}_{l} \rightarrow R^{i}\left(\sigma_{0}^{w}\right)_{!} \overline{\mathbf{Q}}_{l}$ for each $w$. We have

$$
\begin{aligned}
R^{2 d_{\mathbf{m}}^{\prime}}\left(\sigma_{b}\right) ! \overline{\mathbf{Q}}_{l} & \sim R^{2 d_{\mathbf{m}}^{\prime}}\left(\sigma_{0}\right) ! \overline{\mathbf{Q}}_{l}, \\
R^{2 d_{\mathbf{m}}^{\prime}}\left(\sigma_{b}^{w}\right) ! \overline{\mathbf{Q}}_{l} & \stackrel{\sim}{\rightarrow} R^{2 d_{\mathbf{m}}^{\prime}}\left(\sigma_{0}^{w}\right) ! \overline{\mathbf{Q}}_{l} \quad \text { for } w \in \mathcal{W}_{\mathbf{m}}^{\natural} .
\end{aligned}
$$

We show (7.3.1). Let $\widetilde{\alpha}_{0}: \mathcal{Z}_{0}^{(\mathbf{m})} \rightarrow T^{\iota \theta}$ be the restriction of $\widetilde{\alpha}$ on $\widetilde{\mathcal{Z}}_{0}^{(\mathbf{m})}$, and $\alpha_{0}^{w}$ the restriction of $\widetilde{\alpha}_{0}$ on $\widetilde{Z}_{w}$. We also denote by $\alpha_{b}$ (resp. $\alpha_{b}^{w}$ ) the restriciton of $\widetilde{\alpha}_{0}$ on $\mathcal{Z}^{b}$ (resp. on $\mathcal{Z}^{b}(w)$ ). From the compution in the proof of Lemma 7.2, we know that the map $R^{2 d_{\mathbf{m}}^{\prime}}\left(\alpha_{b}^{w}\right) ! \overline{\mathbf{Q}}_{l} \rightarrow R^{2 d_{\mathbf{m}}^{\prime}}\left(\alpha_{0}^{w}\right)_{!} \overline{\mathbf{Q}}_{l}$ is surjective. For any $\mathbf{I} \subset \mathcal{I}\left(\mathbf{m}^{\prime}\right)$ with $\mathbf{m}^{\prime} \leq \mathbf{m}$, we consider $\psi_{\mathbf{I}}: \widetilde{\mathcal{Y}}_{\mathbf{I}} \rightarrow \mathcal{Y}_{\mathbf{m}^{\prime}}^{0}$ as in 1.3. For each $\mathbf{I}, \mathbf{J} \in \mathcal{I}\left(\mathbf{m}^{\prime}\right)$, put $\mathcal{Z}_{\mathbf{I}, \mathbf{J}}=\widetilde{\mathcal{Y}}_{\mathbf{I}} \times_{\mathcal{Y}_{\mathbf{m}}} \widetilde{\mathcal{Y}}_{\mathbf{J}}$ under the inclusion $\mathcal{Y}_{\mathbf{m}^{\prime}}^{0} \hookrightarrow \mathcal{Y}_{\mathrm{m}}$. Note that $\mathcal{Z}_{\mathbf{I}, \mathbf{J}}=\emptyset$ unless $\mathbf{J}=w(\mathbf{I})$ for some $w \in \mathcal{W}_{\mathbf{m}}^{\natural}$. We have a partition $\mathcal{Z}_{0}^{(\mathbf{m})}=\bigsqcup_{\mathbf{I}, \mathbf{J}} \mathcal{Z}_{\mathbf{I}, \mathbf{J}}$ by locally closed subsets $\mathcal{Z}_{\mathbf{I}, \mathbf{J}}$, and $\mathcal{Z}^{\text {b }}$ coincides with $\mathcal{Z}_{\mathbf{I}^{\circ}, \mathbf{I}^{\circ}}$. Let $\mathcal{Z}_{1}$ be the complement of $\mathcal{Z}^{b}$ in $\mathcal{Z}_{0}^{(\mathbf{m})}$, and put $\mathcal{Z}_{1}(w)=\mathcal{Z}_{1} \cap \widetilde{Z}_{w}$. Hence $\mathcal{Z}_{1}=\coprod_{w} \mathcal{Z}_{1}(w)$. Again by a similar computation as in the proof of Lemma 7.2, we see that $\operatorname{dim} \alpha_{w}^{-1}(s) \cap \mathcal{Z}_{1}(w)<d_{\mathbf{m}}^{\prime}$ for any $s \in T^{\iota \theta}$. This implies that $R^{2 d_{\mathbf{m}}^{\prime}}\left(\alpha_{b}^{w}\right) ! \overline{\mathbf{Q}}_{l} \simeq R^{2 d_{\mathbf{m}}^{\prime}}\left(\alpha_{0}^{w}\right)_{!} \overline{\mathbf{Q}}_{l}$. By applying $R^{0}\left(\omega_{T}\right)$ ! on both sides, we have $R^{2 d_{\mathbf{m}}^{\prime}}\left(\sigma_{b}^{w}\right) ! \overline{\mathbf{Q}}_{l} \simeq R^{2 d_{\mathbf{m}}^{\prime}}\left(\sigma_{0}^{w}\right) ! \overline{\mathbf{Q}}_{l}$ for any $w \in \mathcal{W}_{\mathbf{m}}^{\natural}$. This proves the second statement of (7.3.1). By considering the long exact sequence arising from the stratification $\mathcal{Z}_{0}^{(\mathbf{m})}=\coprod_{w}\left(\mathcal{Z}_{0}^{(\mathbf{m})} \cap \widetilde{Z}_{w}\right)$, we obtain the first statement. Thus (7.3.1) holds.

By (7.3.1), the proof of the proposition is reduced to showing

$$
R^{2 d_{\mathbf{m}}^{\prime}}\left(\sigma_{b}\right) ! \overline{\mathbf{Q}}_{l} \simeq \bigoplus_{w \in \mathcal{W}_{\mathbf{m}}^{\natural}} R^{2 d_{\mathbf{m}}^{\prime}}\left(\sigma_{b}^{w}\right) ! \overline{\mathbf{Q}}_{l} .
$$

Note that in this case $\mathcal{Z}_{b} \rightarrow \mathcal{Y}_{\mathbf{m}}^{0}$ is a finite Galois covering. (This is clear in the enhanced case. In the exotic case, since $m_{r}=0$, we have $I_{r}^{\circ}=\emptyset$. Hence $\widetilde{\mathcal{Y}}_{b} \rightarrow \mathcal{Y}_{\mathrm{m}}^{0}$ is a finite Galois covering.) Also note that $\mathcal{Z}^{b}(w)=\emptyset$ unless $w \in \mathcal{W}_{\mathbf{m}}^{\natural}$. In that case, $\mathcal{Z}^{\mathrm{b}}(w)$ is an open and closed subset of $\mathcal{Z}^{\mathrm{b}}$ since $\varphi_{\mathrm{b}}$ is a finite Galois covering. We have a decompsotion $\mathcal{Z}^{b}=\coprod_{w \in \mathcal{W}_{\mathbf{m}}^{\natural}} \mathcal{Z}^{b}(w)$ into open and closed subsets. This implies that

$$
\left(\sigma_{b}\right) ! \overline{\mathbf{Q}}_{l} \simeq \bigoplus_{w \in \mathcal{W}_{\mathbf{m}}^{\natural}}\left(\sigma_{b}^{w}\right)_{!} \overline{\mathbf{Q}}_{l} .
$$

Hence (7.3.2) holds. The proposition is proved.

7.4. By the Künneth formula, $\varphi_{!} \overline{\mathbf{Q}}_{l} \simeq \pi_{!}^{(\mathbf{m})} \overline{\mathbf{Q}}_{l} \otimes \pi_{!}^{(\mathbf{m})} \overline{\mathbf{Q}}_{l}$. By Theorem 3.2 and Theorem 4.5, $\pi_{!}^{(\mathbf{m})} \overline{\mathbf{Q}}_{l}$ has a natural structure of $\mathcal{W}_{\mathbf{m}}^{\natural}$-module. Hence $\varphi_{!} \overline{\mathbf{Q}}_{l}$ has a 
structure of $\mathcal{W}_{\mathbf{m}}^{\natural} \times \mathcal{W}_{\mathbf{m}}^{\natural}$-module. It follows that $\mathcal{T}=\mathcal{H}^{2 d_{\mathbf{m}}^{\prime}}\left(\sigma_{!} \overline{\mathbf{Q}}_{l}\right) \simeq \mathcal{H}^{2 d_{\mathbf{m}}^{\prime}}\left(\widetilde{\omega}_{!}\left(\varphi_{!} \overline{\mathbf{Q}}_{l}\right)\right)$ has a natural action of $\mathcal{W}_{\mathbf{m}}^{\natural} \times \mathcal{W}_{\mathbf{m}}^{\natural}$. Under the decomposition of $\mathcal{T}$ in Proposition 7.3 , the action of $\mathcal{W}_{\mathbf{m}}^{\natural} \times \mathcal{W}_{\mathbf{m}}^{\natural}$ has the following property. For each $w_{1}, w_{2} \in \mathcal{W}_{\mathbf{m}}^{\natural}$,

$$
\left(w_{1}, w_{2}\right) \cdot \mathcal{T}_{w}=\mathcal{T}_{w_{1} w w_{2}^{-1}}
$$

In fact, since $\mathcal{T}$ is a perfect sheaf by Lemma 7.2, it is enough to check (7.4.1) for the restriction of $\mathcal{T}$ on $\Xi_{\text {reg. }}$. Here $\left(\varphi_{b}\right) ! \overline{\mathbf{Q}}_{l}$ has already a structure of $\mathcal{W}_{\mathbf{m}}^{\natural} \times \mathcal{W}_{\mathbf{m}}^{\natural}$-module. Hence by (7.3.1), it is enough to check a similar property for the decomposition of $\left(\varphi_{b}\right)_{!} \overline{\mathbf{Q}}_{l}$ in (7.3.3). But this can be verified directly from the discussion in 7.3. Hence (7.4.1) holds.

Let $a_{0}$ be the element in $\Xi$ corresponding to the $S_{n}$-orbit of $1 \in T^{\iota \theta}$, and $\mathcal{T}_{a_{0}}$ be the stalk of $\mathcal{T}$ at $a_{0} \in \Xi$. By Proposition 7.3, we have a decomposition

$$
\mathcal{T}_{a_{0}}=\bigoplus_{w \in \mathcal{W}_{\mathbf{m}}^{\natural}}\left(\mathcal{T}_{w}\right)_{a_{0}}
$$

where $\left(\mathcal{T}_{w}\right)_{a_{0}}$ is the stalk of $\mathcal{T}_{w}$ at $a_{0}$. $\mathcal{W}_{\mathbf{m}}^{\natural} \times \mathcal{W}_{\mathbf{m}}^{\natural}$ acts on $\mathcal{T}_{a_{0}}$ following (7.4.1). By (7.2.1), $\mathcal{T}_{w} \simeq\left(\omega_{T}\right) ! \overline{\mathbf{Q}}_{l}$. Since $\omega_{T}^{-1}\left(a_{0}\right)=\{1\} \subset T^{\iota \theta},\left(\mathcal{T}_{w}\right)_{a_{0}} \simeq H_{c}^{0}\left(\omega_{T}^{-1}\left(a_{0}\right), \overline{\mathbf{Q}}_{l}\right) \simeq \overline{\mathbf{Q}}_{l}$. Thus we have proved

Proposition 7.5. $\mathcal{T}_{a_{0}}$ has a structure of $\mathcal{W}_{\mathbf{m}}^{\natural} \times \mathcal{W}_{\mathbf{m}}^{\natural}$-module, which coincides with the two-sided regular representation of $\mathcal{W}_{\mathbf{m}}^{\natural}$.

The following lemma is a variant of [Lu, Lemma 6.7].

Lemma 7.6. Let $A, A^{\prime}$ be simple perverse sheaves on $\mathcal{X}_{\mathbf{m} \text {,uni. }}$. Then we have

$$
\operatorname{dim} \mathbf{H}_{c}^{0}\left(\mathcal{X}_{\mathbf{m}, \text { uni }}, A \otimes A^{\prime}\right)= \begin{cases}1 & \text { if } A^{\prime} \simeq D(A), \\ 0 & \text { otherwise }\end{cases}
$$

where $D(A)$ is the Veridier dual of $A$.

Proof. Assume that $A=\operatorname{IC}(\bar{X}, \mathcal{E})[\operatorname{dim} X]$ and $A^{\prime}=\operatorname{IC}\left(\bar{X}^{\prime}, \mathcal{E}^{\prime}\right]\left[\operatorname{dim} X^{\prime}\right]$, where $X, X^{\prime}$ are smooth irreducible subvarieties of $\mathcal{X}_{\mathrm{m} \text {,uni }}$ and $\mathcal{E}$ (resp. $\mathcal{E}^{\prime}$ ) is a simple local system on $X$ (resp. on $\left.X^{\prime}\right)$. We have $\mathbf{H}_{c}^{0}\left(\mathcal{X}_{\text {m,uni }}, A \otimes A^{\prime}\right) \simeq \mathbf{H}_{c}^{0}\left(\bar{X} \cap \bar{X}^{\prime}, A \otimes A^{\prime}\right)$. First assume that $\bar{X} \neq \bar{X}^{\prime}$, and put $Y=\bar{X} \cap \bar{X}^{\prime}$. We show

$$
\mathbf{H}_{c}^{0}\left(Y, A \otimes A^{\prime}\right)=0 .
$$

For this, by using the hypercohomology spectral sequence, it is enough to show the following.

(7.6.2) If $H_{c}^{i}\left(Y, \mathcal{H}^{j} A \otimes \mathcal{H}^{j^{\prime}} A^{\prime}\right) \neq 0$, then $i+j+j^{\prime}<0$.

We show (7.6.2). Suppose that $H_{c}^{i}\left(Y, \mathcal{H}^{j} A \otimes \mathcal{H}^{j^{\prime}} A^{\prime}\right) \neq 0$. Put

$$
Y_{j, j^{\prime}}=\operatorname{supp} \mathcal{H}^{j} A \cap \operatorname{supp} \mathcal{H}^{j^{\prime}} A^{\prime} \subset Y .
$$


We have $H_{c}^{i}\left(Y_{j, j^{\prime}}, \mathcal{H}^{j} A \otimes \mathcal{H}^{j^{\prime}} A^{\prime}\right) \simeq H_{c}^{i}\left(Y, \mathcal{H}^{j} A \otimes \mathcal{H}^{j^{\prime}} A^{\prime}\right) \neq 0$. It follows that $i \leq$ $2 \operatorname{dim} Y_{j, j^{\prime}}$. By using the property of intersection cohomology, we have

$$
\begin{aligned}
& \operatorname{dim} Y_{j, j^{\prime}} \leq \operatorname{dim} \operatorname{supp} \mathcal{H}^{j} A \leq-j, \\
& \operatorname{dim} Y_{j, j^{\prime}} \leq \operatorname{dim} \operatorname{supp} \mathcal{H}^{j^{\prime}} A^{\prime} \leq-j^{\prime}
\end{aligned}
$$

and so

$$
j \leq-\operatorname{dim} Y_{j, j^{\prime}}, \quad j^{\prime} \leq-\operatorname{dim} Y_{j, j^{\prime}} .
$$

Since $\bar{X} \neq \bar{X}^{\prime}$, we have $\operatorname{dim} Y<\operatorname{dim} X$ or $\operatorname{dim} Y<\operatorname{dim} X^{\prime}$, and one of the inequalities in (7.6.3) is a strict inequality. It follows that $i+j+j^{\prime}<0$. Hence (7.6.2) holds and (7.6.1) follows.

Next assume that $\bar{X}=\bar{X}^{\prime}$. We may assume that $X, X^{\prime}$ are open dense in $\bar{X}$. By replacing $X, X^{\prime}$ by $X \cap X^{\prime}$, if necessary, we may assume that $X=X^{\prime}$. Put $Y=\bar{X} \backslash X$. We show that

$$
\mathbf{H}_{c}^{0}\left(Y, A \otimes A^{\prime}\right)=0 \text { and } \mathbf{H}_{c}^{-1}\left(Y, A \otimes A^{\prime}\right)=0 .
$$

As in the previous case, we consider the hypercohomology spectral sequence. Suppose that $H_{c}^{i}\left(Y, \mathcal{H}^{j} A \otimes \mathcal{H}^{j^{\prime}} A^{\prime}\right) \neq 0$. We may replace $Y$ by $Y_{j, j^{\prime}}$, where $Y_{j, j^{\prime}}=$ $Y \cap \operatorname{supp} \mathcal{H}^{j} A \cap \operatorname{supp} \mathcal{H}^{j^{\prime}} A^{\prime}$. Then we have $i \leq 2 \operatorname{dim} Y_{j, j^{\prime}}$, and we obtain a similar formula as (7.6.3), but in this case, both of them are strict inequalities since $\operatorname{dim} Y<\operatorname{dim} X$. It follows that $i+j+j^{\prime}<-1$. This proves (7.6.4). By using the cohomology long exaxt sequence with respect to $Y=\bar{X} \backslash X$, we see that

$$
\mathbf{H}_{c}^{0}\left(\bar{X}, A \otimes A^{\prime}\right) \simeq \mathbf{H}_{c}^{0}\left(X, A \otimes A^{\prime}\right) \simeq H_{c}^{2 \operatorname{dim} X}\left(X, \mathcal{E} \otimes \mathcal{E}^{\prime}\right) .
$$

The last space is isomorphic to $\overline{\mathbf{Q}}_{l}$ if $\mathcal{E}^{\prime} \simeq \mathcal{E}^{\vee}$, the dual local system, and is equal to zero otherwise. Since $D(A)=\operatorname{IC}\left(\bar{X}, \mathcal{E}^{\vee}\right)[\operatorname{dim} X]$, the lemma is proved.

7.7. We consider the map $\pi_{1}^{(\mathbf{m})}: \widetilde{\mathcal{X}}_{\mathbf{m}, \text { uni }} \rightarrow \mathcal{X}_{\mathbf{m} \text {,uni. }}$ Put $K_{\mathbf{m}, 1}=\left(\pi_{1}^{(\mathbf{m})}\right)_{!} \overline{\mathbf{Q}}_{l}\left[d_{\mathbf{m}}^{\prime}\right]$. By Lemma 6.21 (iii), the map $\pi_{1}^{(\mathbf{m})}$ is semi-small. Hence $K_{\mathbf{m}, 1}$ is a semisimple perverse sheaf on $\mathcal{X}_{\mathrm{m} \text {,uni }}$ and is decomposed as

$$
K_{\mathbf{m}, 1} \simeq \bigoplus_{A \in \mathcal{C}_{\mathbf{m}}} V_{A} \otimes A
$$

where $\mathcal{C}_{\mathbf{m}}$ is the set of (isomorphism classes of) simple perverse sheaves appearing in the decomposition of $K_{\mathbf{m}, 1}$, and $V_{A}=\operatorname{Hom}\left(K_{\mathrm{m}, 1}, A\right)$ is the multiplicity space for $A$. We have the following.

Proposition 7.8. Under the notation as above, put $m_{A}=\operatorname{dim} V_{A}$ for each $A \in \mathcal{C}_{\mathbf{m}}$. Then we have

$$
\sum_{A \in \mathcal{C}_{\mathbf{m}}} m_{A}^{2}=\left|\mathcal{W}_{\mathbf{m}}^{\natural}\right|
$$


Proof. By the computation in 7.4, we have

$$
\begin{aligned}
\mathcal{T}_{a_{0}} & \simeq \mathbf{H}_{c}^{2 d_{\mathbf{m}}^{\prime}}\left(\mathcal{X}_{\mathbf{m}, \text { uni }},\left(\pi_{1}^{(\mathbf{m})}\right)_{!} \overline{\mathbf{Q}}_{l} \otimes\left(\pi_{1}^{(\mathbf{m})}\right)_{!} \overline{\mathbf{Q}}_{l}\right) \\
& \simeq \mathbf{H}_{c}^{0}\left(\mathcal{X}_{\mathbf{m}, \text { uni }}, K_{\mathbf{m}, 1} \otimes K_{\mathbf{m}, 1}\right) .
\end{aligned}
$$

Hence by (7.7.1), we have

$$
\operatorname{dim} \mathcal{T}_{a_{0}}=\sum_{A, A^{\prime} \in \mathcal{C}_{\mathbf{m}}}\left(m_{A} m_{A^{\prime}}\right) \operatorname{dim} \mathbf{H}_{c}^{0}\left(\mathcal{X}_{\mathbf{m}, \text { uni }}, A \otimes A^{\prime}\right)
$$

By Lemma $7.6, \mathbf{H}_{c}^{0}\left(\mathcal{X}_{\mathbf{m}, \text { uni }}, A \otimes A^{\prime}\right) \neq 0$ only when $D(A)=A^{\prime}$, in which case, $\operatorname{dim} \mathbf{H}_{c}^{0}\left(\mathcal{X}_{\mathbf{m}, \text { uni }}, A \otimes A^{\prime}\right)=1$. But since $K_{\mathbf{m}, 1}$ is self dual, $m_{A}=m_{D(A)}$ for each $A$. It follows that $\operatorname{dim} \mathcal{T}_{a_{0}}=\sum_{A \in \mathcal{C}_{\mathrm{m}}} m_{A}^{2}$. On the other hand, by Proposition 7.5, we have $\operatorname{dim} \mathcal{T}_{a_{0}}=\left|\mathcal{W}_{\mathbf{m}}^{\natural}\right|$. This proves the proposition.

7.9. Since $\pi_{!}^{(\mathbf{m})} \overline{\mathbf{Q}}_{l}$ is equipped with the $\mathcal{W}_{\mathbf{m}}^{\natural}$-action, for each $z=(x, \mathbf{v}) \in$ $\mathcal{X}_{\mathbf{m}}, H^{i}\left(\left(\pi^{(\mathbf{m})}\right)^{-1}(z), \overline{\mathbf{Q}}_{l}\right)$ turns out to be a $\mathcal{W}_{\mathbf{m}}^{\natural}$-module. In the case where $z_{0}=$ $(1, \mathbf{0}),\left(\pi^{(\mathbf{m})}\right)^{-1}\left(z_{0}\right) \simeq H / B^{\theta}$ and so $H^{i}\left(H / B^{\theta}, \overline{\mathbf{Q}}_{l}\right)$ has a structure of $\mathcal{W}_{\mathbf{m}}^{\natural}$-module. Note that $\mathcal{W}_{\mathbf{m}}^{\natural}$ is a subgroup of $W_{n}$ (resp. of $S_{n}$ ) in the exotic case (resp. the enhanced case). It is well-known that the Weyl group $W_{n}$ (resp. $S_{n}$ ) acts naturally on $H^{i}\left(H / B^{\theta}, \overline{\mathbf{Q}}_{l}\right)$, which we call the classical action of $W_{n}\left(\operatorname{resp} . S_{n}\right)$ on it. We have the following lemma.

Lemma 7.10. The action of $\mathcal{W}_{\mathbf{m}}^{\natural}$ on $H^{i}\left(H / B^{\theta}, \overline{\mathbf{Q}}_{l}\right)$ coincides with the restriction of the classical action of $W_{n}$ (resp. $\left.S_{n}\right)$ on it in the exotic case (resp. the enhanced case).

Proof. First assume that $\mathcal{X}_{\mathbf{m}}$ is of exotic type. Put $\mathbf{m}^{\prime}=(0, \ldots, 0, n, 0) \in \mathcal{Q}_{n, r}^{0}$. Then $\mathcal{Y}_{\mathbf{m}^{\prime}} \subset \mathcal{Y}_{\mathbf{m}}$, and $\mathcal{X}_{\mathbf{m}^{\prime}} \subset \mathcal{X}_{\mathbf{m}}$ (in fact, $\mathcal{Y}_{\mathbf{m}^{\prime}}=\left\{(x, \mathbf{v}) \in \mathcal{Y}_{\mathbf{m}} \mid v_{1}=\cdots=v_{r-2}=0\right\}$ and similarly for $\left.\mathcal{X}_{\mathbf{m}^{\prime}}\right)$. We have $\mathcal{W}_{\mathbf{m}^{\prime}}^{\natural}=W_{n}$. In this case, $\left.\psi_{!}^{(\mathbf{m})} \overline{\mathbf{Q}}_{l}\right|_{\mathcal{Y}_{\mathbf{m}^{\prime}}} \simeq \psi_{!}^{\left(\mathbf{m}^{\prime}\right)} \overline{\mathbf{Q}}_{l}$, and $\left.\pi_{!}^{(\mathbf{m})} \overline{\mathbf{Q}}_{l}\right|_{\mathcal{X}_{\mathbf{m}^{\prime}}} \simeq \pi_{!}^{\left(\mathbf{m}^{\prime}\right)} \overline{\mathbf{Q}}_{l}$. It follows from the construction that the action of $\mathcal{W}_{\mathbf{m}}^{\natural}$ on $\left.\psi_{!}^{(\mathbf{m})} \overline{\mathbf{Q}}_{l}\right|_{\mathcal{Y}_{\mathbf{m}^{\prime}}}$ coincides with the restriciton of the action of $\mathcal{W}_{\mathbf{m}^{\prime}}^{\natural} \simeq W_{n}$ on $\psi_{!}^{\left(\mathbf{m}^{\prime}\right)} \overline{\mathbf{Q}}_{l}$. Hence a similar fact holds also for $\pi_{!}^{(\mathbf{m})} \overline{\mathbf{Q}}_{l}$. In particular, the $\mathcal{W}_{\mathbf{m}}^{\natural}$-action on $\mathcal{H}_{z_{0}}^{i}\left(\pi_{!}^{(\mathbf{m})} \overline{\mathbf{Q}}_{l}\right)$ coincides with the restriction of the $\mathcal{W}_{\mathbf{m}^{\prime}}^{\natural}$-action on $\mathcal{H}_{z_{0}}^{i}\left(\pi_{!}^{\left(\mathbf{m}^{\prime}\right)} \overline{\mathbf{Q}}_{l}\right)$. Here $\mathcal{X}_{\mathbf{m}^{\prime}} \simeq G^{\iota \theta} \times V$, and $\pi_{!}^{\left(\mathbf{m}^{\prime}\right)} \overline{\mathbf{Q}}_{l}$ is isomorphic to $\pi_{!} \overline{\mathbf{Q}}_{l}$, where $\pi: \widetilde{\mathcal{X}} \rightarrow \mathcal{X}=G^{\iota \theta} \times V$ is the map defined in 1.2 for the case $r=2$. The complex $\pi_{!} \overline{\mathbf{Q}}_{l}$ was studied in [SS1], and one sees that the action of $\mathcal{W}_{\mathbf{m}^{\prime}}^{\natural}$ on $\pi_{!}^{\left(\mathbf{m}^{\prime}\right)} \overline{\mathbf{Q}}_{l}$ is nothing but the $W_{n}$-action on $\pi_{!} \overline{\mathbf{Q}}_{l}$ constructed in $[\mathrm{SS} 1]$. It induces a $W_{n}$-action on $H^{i}\left(H / B^{\theta}, \overline{\mathbf{Q}}_{l}\right)$, which is called the exotic action of $W_{n}$. Hence in order to prove the lemma, it is enough to see that the exotic action and the classical action of $W_{n}$ on $H^{i}\left(H / B^{\theta}, \overline{\mathbf{Q}}_{l}\right)$ coincide with each other. But this is proved in [SS1, Lemma 5.2]. Hence the lemma is proved in the exotic case.

Next assume that $\mathcal{X}_{\mathbf{m}}$ is of enhanced type. In this case, we consider $\mathcal{Y}_{\mathbf{m}^{\prime}} \subset \mathcal{Y}_{\mathbf{m}}$ and $\mathcal{X}_{\mathbf{m}^{\prime}} \subset \mathcal{X}_{\mathbf{m}}$ for $\mathbf{m}^{\prime}=(0, \ldots, 0, n) \in \mathcal{Q}_{n, r}$. Then $\mathcal{X}_{\mathbf{m}^{\prime}} \simeq G^{\iota \theta} \times V$, and as in the exotic case, the proof is reduced to the case where $r=2$. So we consider $\mathcal{X}=G \times V$ 
for $G=G L(V)$ and let $\pi: \widetilde{\mathcal{X}} \rightarrow \mathcal{X}$ be the corresponding map. We have an action of $S_{n}$ on $H^{i}\left(G / B, \overline{\mathbf{Q}}_{l}\right)$, called the enhanced action of $S_{n}$, obtained from the $S_{n}$ action on $\pi_{!} \overline{\mathbf{Q}}_{l}$. One can prove that the enhanced action coincides with the classical action, by a similar (but simpler) argument as in the proof of Lemma 5.2 in [SS1]. Hence the lemma holds for the enhanced case.

7.11. We keep the assumption as before. By applying Theorem 3.2 and Theorem 4.5 to the case where $\mathcal{E}=\overline{\mathbf{Q}}_{l}$, one can write as

$$
\pi_{!}^{(\mathbf{m})} \overline{\mathbf{Q}}_{l}\left[d_{\mathbf{m}}\right] \simeq \bigoplus_{\rho \in\left(\mathcal{W}_{\mathbf{m}}^{\natural}\right)^{\wedge}} \rho \otimes K_{\rho}
$$

where $d_{\mathbf{m}}=\operatorname{dim} \mathcal{X}_{\mathbf{m}}, K_{\rho}$ is a simple perverse sheaf on $\mathcal{X}_{\mathbf{m}}$ as given in Theorem 3.2 and Theorem 4.5. More precisely, if $\mathcal{X}_{\mathbf{m}}$ is of exotic type then $K_{\rho}=$ $\operatorname{IC}\left(\mathcal{X}_{\mathbf{m}(k)}, \mathcal{L}_{\rho_{1}}\right)\left[d_{\mathbf{m}(k)}\right]$ for $\rho \simeq V_{\rho_{1}}^{\natural}$ with $\rho_{1} \in \mathcal{W}_{\mathbf{m}(k)}^{\wedge}$, and if $\mathcal{X}_{\mathbf{m}}$ is of enhanced type then $K_{\rho}=\operatorname{IC}\left(\mathcal{X}_{\mathbf{m}}, \mathcal{L}_{\rho}\right)\left[d_{\mathbf{m}}\right]$. We consider the complex $\left(\pi_{1}^{(\mathbf{m})}\right)_{!} \overline{\mathbf{Q}}_{l}\left[d_{\mathbf{m}}^{\prime}\right]$, where $d_{\mathbf{m}^{\prime}}=\operatorname{dim} \mathcal{X}_{\mathbf{m} \text {,uni. }}$. The following result gives the Springer correspondence with respect to the action of $\mathcal{W}_{\mathbf{m}}^{\natural}$. In the exotic case, this result is regarded as a weak version of the Springer correspondence.

Theorem 7.12 (Springer correspondence for $\mathcal{W}_{\mathbf{m}}^{\natural}$ ). Let $\mathcal{X}_{\mathbf{m}}$ be of exotic type or of enhanced type for $\mathbf{m} \in \mathcal{Q}_{n, r}$. In the exotic case, assume further that $\mathbf{m} \in \mathcal{Q}_{n, r}^{0}$. Then $\left(\pi_{1}^{(\mathbf{m})}\right)_{!} \overline{\mathbf{Q}}_{l}\left[d_{\mathbf{m}}^{\prime}\right]$ is a semisimple perverse sheaf on $\mathcal{X}_{\mathbf{m} \text {,uni }}$ equipped with $\mathcal{W}_{\mathbf{m}}^{\natural}$-action, and is decomposed as

$$
\left(\pi_{1}^{(\mathbf{m})}\right)_{!} \overline{\mathbf{Q}}_{l}\left[d_{\mathbf{m}}^{\prime}\right] \simeq \bigoplus_{\rho \in\left(\mathcal{W}_{\mathbf{m}}^{\natural}\right)^{\wedge}} \rho \otimes L_{\rho}
$$

where $L_{\rho}$ is a simple perverse sheaf on $\mathcal{X}_{\mathbf{m}, \text { uni }}$ such that

$$
\left.K_{\rho}\right|_{\mathcal{X}_{\mathrm{m}, \mathrm{uni}}} \simeq L_{\rho}\left[d_{\mathbf{m}}-d_{\mathbf{m}}^{\prime}\right]
$$

Proof. As discussed in 7.7, $K_{\mathbf{m}, 1}=\left(\pi_{1}^{(\mathbf{m})}\right)_{!} \overline{\mathbf{Q}}_{l}\left[d_{\mathbf{m}}^{\prime}\right]$ is a semisimple perverse sheaf. Since $K_{\mathbf{m}, 1}$ is the restriction of $\left(\pi^{(\mathbf{m})}\right) ! \overline{\mathbf{Q}}_{l}$ on $\mathcal{X}_{\mathbf{m}, \text { uni }}, K_{\mathbf{m}, 1}$ has a strucute of $\mathcal{W}_{\mathbf{m}}^{\natural}$ module. Thus we can define an algeba homomorphism

$$
\overline{\mathbf{Q}}_{l}\left[\mathcal{W}_{\mathbf{m}}^{\natural}\right] \stackrel{\alpha}{\longrightarrow} \text { End } K_{\mathbf{m}, 1} \stackrel{\beta}{\longrightarrow} \text { End } H^{\bullet}\left(H / B^{\theta}, \overline{\mathbf{Q}}_{l}\right) .
$$

In order to show (7.12.1), it is enough to see that $\alpha$ gives an isomorphism

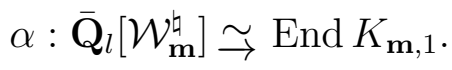

We show (7.12.3). We assume that $\mathcal{X}_{\mathbf{m}}$ is of exotic type. The proof for the enhanced case is similar. $\beta \circ \alpha$ is a homomorphism induced from the action of $\mathcal{W}_{\mathbf{m}}^{\natural}$ on $H^{\bullet}\left(H / B^{\theta}, \overline{\mathbf{Q}}_{l}\right)$. By Lemma 7.10 , this action is the restriction of the classical 
action of $W_{n}$ on $H^{\bullet}\left(H / B^{\theta}, \overline{\mathbf{Q}}_{l}\right)$. Since $H^{\bullet}\left(H / B^{\theta}, \overline{\mathbf{Q}}_{l}\right) \simeq \overline{\mathbf{Q}}_{l}\left[W_{n}\right]$ as $W_{n}$-module, with respect to the classical action, $\overline{\mathbf{Q}}_{l}\left[W_{n}\right] \rightarrow H^{\bullet}\left(H / B^{\theta}, \overline{\mathbf{Q}}_{l}\right)$ is injective. Hence $\beta \circ \alpha$ is injective. It follows that $\alpha$ is injective. On the other hand, Proposition 7.8 implies that $\operatorname{dim}$ End $K_{\mathbf{m}, 1}=\left|\mathcal{W}_{\mathbf{m}}^{\natural}\right|$. This shows that $\alpha$ is surjective, and so (7.12.3) holds.

(7.12.2) now follows easily by comparing (7.11.1) and (7.12.1). The theorem is proved.

7.13. We now return to the setting in 1.6 , and consider the complex reflection group $W_{n, r}$. For each $\mathbf{m} \in \mathcal{Q}_{n, r}^{0}$, we denote by $\left(W_{n, r}^{\wedge}\right)_{\mathbf{m}}$ the set of irreducible representations $\widetilde{V}_{\rho}$ (up to isomorphism) of $W_{n, r}$ obtained from $\rho \in \mathcal{W}_{\mathbf{m}(k)}^{\wedge}$ for various $0 \leq k \leq m_{r-1}$ as in 1.6. Then we have

$$
W_{n, r}^{\wedge}=\coprod_{\mathbf{m} \in \mathcal{Q}_{n, r}^{0}}\left(W_{n, r}^{\wedge}\right)_{\mathbf{m}}
$$

It follows from the construction of $\widetilde{V}_{\rho}$ and of $V_{\rho}^{\natural}$, there exists a natual bijection between $\left(W_{n, r}^{\wedge}\right)_{\mathbf{m}}$ and $\left(W_{\mathbf{m}}^{\natural}\right)^{\wedge}$. We denote by $V(\rho)$ the irreducible representation of $W_{n, r}$ belonging to $\left(W_{n, r}^{\wedge}\right)_{\mathbf{m}}$ corresponding to $\rho \in\left(W_{\mathbf{m}}^{\natural}\right)^{\wedge}$. Now assume that $\mathcal{X}_{\mathbf{m}}$ is of exotic type. We consider the map $\bar{\pi}_{\mathrm{m}}: \pi^{-1}\left(\mathcal{X}_{\mathrm{m}}\right) \rightarrow \mathcal{X}_{\mathrm{m}}$, and the complex $\left(\bar{\pi}_{\mathbf{m}}\right) ! \overline{\mathbf{Q}}_{l}\left[d_{\mathbf{m}}\right]$ as in 2.1. Then by Theorem $2.2,\left(\bar{\pi}_{\mathbf{m}}\right)_{!} \overline{\mathbf{Q}}_{l}\left[d_{\mathbf{m}}\right]$ is a semisimple perverse

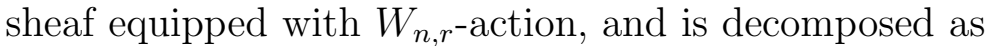

$$
\left(\bar{\pi}_{\mathbf{m}}\right)_{!} \overline{\mathbf{Q}}_{l}\left[d_{\mathbf{m}}\right] \simeq \bigoplus_{\rho \in\left(W_{\mathbf{m}}^{\natural}\right)^{\wedge}} V(\rho) \otimes K_{\rho},
$$

where $K_{\rho}$ is a simple perverse sheaf on $\mathcal{X}_{\mathbf{m}}$ given in (7.11.1). Let $\bar{\pi}_{\mathbf{m}, 1}: \pi^{-1}\left(\mathcal{X}_{\mathbf{m}, \text { uni }}\right) \rightarrow$ $\mathcal{X}_{\mathbf{m} \text {,uni }}$ be the restriciton of $\bar{\pi}_{\mathbf{m}}$ on $\mathcal{X}_{\mathbf{m} \text {,uni. }}$. Since $\bar{\pi}_{\mathbf{m}, 1}$ is proper, $\bar{\pi}_{\mathbf{m}, 1} \overline{\mathbf{Q}}_{l}\left[d_{\mathbf{m}}^{\prime}\right]$ is a semisimple complex on $\mathcal{X}_{\mathbf{m} \text {,uni }}$. By applying $(7.12 .2)$, we see that $\bar{\pi}_{\mathbf{m}, 1} \overline{\mathbf{Q}}_{l}\left[d_{\mathbf{m}}^{\prime}\right]$ is a semisimple perverse sheaf. As a corollary to Theorem 7.12, we obtain the Springer correspondence for $W_{n, r}$.

Corollary 7.14 (Springer correspondence for $W_{n, r}$ ). Assume that $\mathcal{X}_{\mathbf{m}}$ is of exotic type with $\mathbf{m} \in \mathcal{Q}_{n, r}^{0}$. Then $\bar{\pi}_{\mathbf{m}, 1} \overline{\mathbf{Q}}_{l}\left[d_{\mathbf{m}}^{\prime}\right]$ is a semisimple perverse sheaf on $\mathcal{X}_{\mathbf{m} \text {,uni }}$ with $W_{n, r}$-action, and is decomposed as

$$
\bar{\pi}_{\mathbf{m}, 1} \overline{\mathbf{Q}}_{l}\left[d_{\mathbf{m}}^{\prime}\right] \simeq \bigoplus_{\rho \in\left(W_{\mathbf{m}}^{\natural}\right)^{\wedge}} V(\rho) \otimes L_{\rho},
$$

where $L_{\rho}$ is a simple perverse sheaf on $\mathcal{X}_{\mathbf{m} \text {,uni }}$ as in Theorem 7.12 , and $V(\rho)$ is an irreducible representation of $W_{n, r}$ as defined in 7.13 .

\section{Determination of the Springer correspondence}

8.1. Assume that $r \geq 2$. In this section we shall determine $L_{\rho}$ appearing in the Springer correspondence explicitly. For a fixed $\mathbf{m} \in \mathcal{Q}_{n, r}$, we consider $\mathcal{X}_{\mathbf{m}}$ of exotic 
type or of enhanced type. In the exotic case, we assume that $\mathbf{m} \in \mathcal{Q}_{n, r}^{0}$. First we consider the case where $\mathcal{X}_{\mathbf{m}}$ is of exotic type. By modifiying the definition of $\mathcal{K}_{\mathbf{m}}$ in 2.5 , we define a variety $\mathcal{G}_{\mathrm{m}}$ by

$$
\begin{array}{r}
\mathcal{G}_{\mathbf{m}}=\left\{\left(x, \mathbf{v},\left(W_{i}\right)_{1 \leq i \leq r-2}\right) \mid(x, \mathbf{v}) \in \mathcal{X},\left(W_{i}\right): \text { partial isotropic flag in } V\right. \\
\left.\operatorname{dim} W_{i}=p_{i}, x\left(W_{i}\right)=W_{i}, v_{i} \in W_{i}(1 \leq i \leq r-2), v_{r-1} \in W_{r-2}^{\perp}\right\} .
\end{array}
$$

Let $\zeta: \mathcal{G}_{\mathrm{m}} \rightarrow \mathcal{X}$ be the projection to the first two factors. We consider the map $\pi^{(\mathbf{m})}: \widetilde{\mathcal{X}}_{\mathbf{m}} \rightarrow \mathcal{X}_{\mathbf{m}}$. Then $\pi^{(\mathbf{m})}$ is decomposed as

$$
\pi^{(\mathbf{m})}: \widetilde{\mathcal{X}}_{\mathbf{m}} \stackrel{\varphi}{\longrightarrow} \mathcal{G}_{\mathbf{m}} \stackrel{\zeta}{\longrightarrow} \mathcal{X}_{\mathbf{m}}
$$

where $\varphi$ is defined by $\left(x, \mathbf{v}, g B^{\theta}\right) \mapsto\left(x, \mathbf{v},\left(g M_{p_{i}}\right)_{1 \leq i \leq r-2}\right)$. The map $\varphi$ is surjective since $\mathbf{m} \in \mathcal{Q}_{n, r}^{0}$. It follows that $\zeta\left(\mathcal{G}_{\mathbf{m}}\right)$ coincides with $\mathcal{X}_{\mathbf{m}}$. Since $\mathbf{m} \in \mathcal{Q}_{n, r}^{0}$, we have $\operatorname{dim} \widetilde{\mathcal{X}}_{\mathbf{m}}=\operatorname{dim} \mathcal{X}_{\mathbf{m}}$. This implies that $\operatorname{dim} \mathcal{G}_{\mathbf{m}}=\operatorname{dim} \mathcal{X}_{\mathbf{m}}$. Note that in the case where $r=2$, we have $\mathbf{m}=(n, 0)$, and $\mathcal{G}_{\mathbf{m}}=G^{\iota \theta} \times V=\mathcal{X}_{\mathbf{m}}$. We define a variety $\mathcal{H}_{\mathrm{m}}$ as in 2.5 by

$$
\begin{aligned}
\mathcal{H}_{\mathbf{m}}=\left\{\left(x, \mathbf{v},\left(W_{i}\right), \phi_{1}, \phi_{2}\right) \mid\left(x, \mathbf{v},\left(W_{i}\right)\right) \in \mathcal{G}_{\mathbf{m}}\right. \\
\left.\phi_{1}: W_{1} \stackrel{\sim}{\rightarrow} V_{0}, \phi_{2}: W_{1}^{\perp} / W_{1} \underset{\rightarrow}{\rightarrow} \bar{V}_{0} \text { (symplectic isom.) }\right\},
\end{aligned}
$$

where $V_{0}=M_{p_{1}}$ and $\bar{V}_{0}=M_{p_{1}}^{\perp} / M_{p_{1}}$. We also define a variety $\widetilde{\mathcal{Z}}_{\mathrm{m}}$ by

$$
\begin{aligned}
\widetilde{\mathcal{Z}}_{\mathbf{m}}=\left\{\left(x, \mathbf{v}, g B^{\theta}, \phi_{1}, \phi_{2}\right)\right. & \mid\left(x, \mathbf{v}, g B^{\theta}\right) \in \widetilde{\mathcal{X}}_{\mathbf{m}}, \\
\phi_{1}: g\left(M_{p_{1}}\right) & \left.\stackrel{\sim}{\rightarrow} V_{0}, \phi_{2}: g\left(M_{p_{1}}\right)^{\perp} / g\left(M_{p_{1}}\right) \stackrel{\sim}{\rightarrow} \bar{V}_{0}\right\} .
\end{aligned}
$$

Assume that $r \geq 3$, and let $\mathbf{m}^{\prime}=\left(m_{2}, \ldots, m_{r}\right)$ for $\mathbf{m}=\left(m_{1}, \ldots, m_{r}\right)$, and $G_{1}, \widetilde{G}_{1}$, $\mathcal{X}_{\mathbf{m}^{\prime}}^{\prime}, G_{2}$, etc. be as in 2.5. $\widetilde{\mathcal{X}}_{\mathbf{m}^{\prime}}^{\prime}$ is defined for $\mathcal{X}_{\mathbf{m}^{\prime}}^{\prime}$ in a similar way as $\widetilde{\mathcal{X}}_{\mathbf{m}}$ is defined for $\mathcal{X}_{\mathbf{m}}$. (Hence in the case where $r=3, \mathcal{X}_{\mathbf{m}^{\prime}}^{\prime}=\mathcal{G}_{\mathbf{m}^{\prime}}^{\prime}=G_{2}^{\iota \theta} \times \bar{V}_{0}$.) We have the following commutative diagram

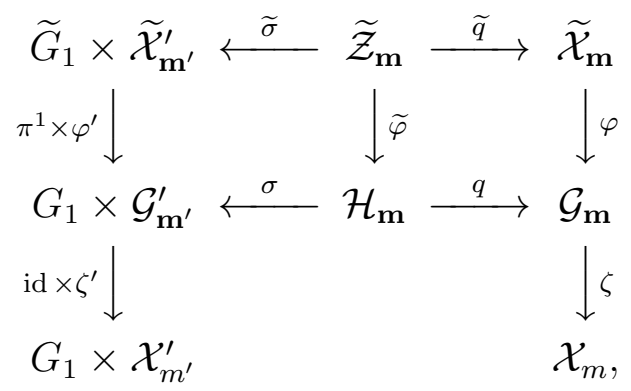

where $q, \sigma$ are defined in a similar way as in 2.5, and

$$
\widetilde{q}:\left(x, \mathbf{v}, g B^{\theta}, \phi_{1}, \phi_{2}\right) \mapsto\left(x, \mathbf{v}, g B^{\theta}\right),
$$




$$
\widetilde{\varphi}:\left(x, \mathbf{v}, g B^{\theta}, \phi_{1}, \phi_{2}\right) \mapsto\left(x, \mathbf{v},\left(g M_{p_{i}}\right)_{1 \leq i \leq r-2}, \phi_{1}, \phi_{2}\right)
$$

The map $\widetilde{\sigma}$ is defined as follows. Let $\mathcal{F}^{\theta}(V)$ be the set of isotropic flags in $V$, and $\widetilde{\mathcal{F}}_{\mathbf{m}}^{\theta}$ the set of $\left((x, \mathbf{v}),\left(V_{i}\right)\right) \in \mathcal{X}_{\mathbf{m}} \times \mathcal{F}^{\theta}(V)$ such that $\left(V_{i}\right)$ is $x$-stable and that $v_{i} \in V_{p_{i}}$ for $i=1, \ldots, r-1$. Then $\widetilde{\mathcal{F}}_{\mathbf{m}}^{\theta}$ is isomorphic to $\widetilde{\mathcal{X}}_{\mathbf{m}}$, and

$$
\begin{aligned}
\widetilde{\mathcal{Z}}_{\mathbf{m}} \simeq\left\{\left(x, \mathbf{v},\left(V_{i}\right), \phi_{1}, \phi_{2}\right) \mid\right. & \left(x, \mathbf{v},\left(V_{i}\right)\right) \in \widetilde{\mathcal{F}}_{\mathbf{m}}^{\theta}, \\
\phi_{1}: V_{p_{1}} & \left.\sim V_{0}, \phi_{2}: V_{p_{1}}^{\perp} / V_{p_{1}} \sim \bar{V}_{0}\right\} .
\end{aligned}
$$

We define $\widetilde{\sigma}: \widetilde{\mathcal{Z}}_{\mathbf{m}} \rightarrow \widetilde{G}_{1} \times \widetilde{\mathcal{X}}_{\mathbf{m}^{\prime}}^{\prime}$ by $\left(x, \mathbf{v},\left(V_{i}\right), \phi_{1}, \phi_{2}\right) \mapsto\left(\xi_{1}, \xi_{2}\right)$ with

$$
\begin{aligned}
& \xi_{1}=\left(\phi_{1}\left(x^{\prime}\right) \phi_{1}^{-1},\left(\phi_{1}\left(V_{i}\right)\right)_{i \leq p_{1}}\right) \in \widetilde{G}_{1} \\
& \xi_{2}=\left(\phi_{2}\left(x^{\prime \prime}\right) \phi_{2}^{-1}, \phi_{2}(\overline{\mathbf{v}}),\left(\phi_{2}\left(V_{i} / V_{p_{1}}\right)\right)_{i \geq p_{1}}\right) \in{\widetilde{\mathcal{X}_{\mathbf{m}^{\prime}}^{\prime}}}^{\prime}
\end{aligned}
$$

where $x^{\prime}$ (resp. $\left.x^{\prime \prime}\right)$ is the restriction of $x$ on $V_{p_{1}}$ (resp. $\left.V_{p_{1}}^{\perp} / V_{p_{1}}\right), \overline{\mathbf{v}}=\left(\bar{v}_{2}, \ldots, \bar{v}_{r-1}\right)$ with $\bar{v}_{i}$ the image of $v_{i}$ on $V_{p_{1}}^{\perp} / V_{p_{1}}$ for $\mathbf{v}=\left(v_{1}, \ldots, v_{r-1}\right)$. One can check that squares in the diagram are both cartesian squares. Put $H_{0}=G_{1} \times G_{2}^{\theta}$. Then as in (2.5.2), and (2.5.3), we have

(8.1.2) $q$ is a principal bundle with fibre isomorphic to $H_{0}$, and $\sigma$ is a locally trivial fibration with smooth fibre of dimension $\operatorname{dim} H+(r-2) m_{1}$.

8.2 For a fixed $k$, we consider the variety $\tilde{\mathcal{Y}}_{\mathbf{m}(k)}^{\dagger}=\left(\psi^{(\mathbf{m})}\right)^{-1}\left(\mathcal{Y}_{\mathbf{m}(k)}^{0}\right)$ as in 3.3. Let $\mathcal{G}_{\mathbf{m}(k) \text {,reg }}=\zeta^{-1}\left(\mathcal{Y}_{\mathbf{m}(k)}^{0}\right)$ be a locally closed subvariety of $\mathcal{G}_{\mathbf{m}}$ (not of $\mathcal{G}_{\mathbf{m}(k)}$, note that $\mathcal{G}_{\mathbf{m}(k)}$ is not defined since $\left.\mathbf{m}(k) \notin \mathcal{Q}_{m, r}^{0}\right)$. We define $\mathbf{m}^{\prime}(k)=\mathbf{m}^{\prime}(r-2, k)$ similar to $\mathbf{m}(k)$, by replacing $\mathbf{m}$ by $\mathbf{m}^{\prime}$. Then the varieties $\mathcal{Y}_{\mathbf{m}^{\prime}(k)}^{\prime 0}, \widetilde{\mathcal{Y}}_{\mathbf{m}^{\prime}(k)}^{\prime \dagger}$ and $\mathcal{G}_{\mathbf{m}^{\prime}(k), \text { reg }}^{\prime}$ are defined similarly with respect to $\mathcal{X}_{\mathbf{m}^{\prime}}^{\prime}$. The commutative diagram (8.1.1) induces a commutative diagram

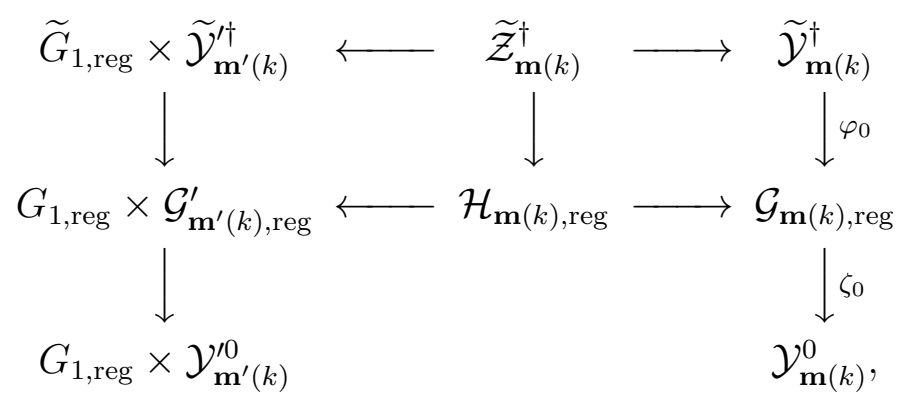

where $\mathcal{H}_{\mathbf{m}(k) \text {,reg }}=q^{-1}\left(\mathcal{G}_{\mathbf{m}(k), \text { reg }}\right), \widetilde{\mathcal{Z}}_{\mathbf{m}(k)}^{\dagger}=\widetilde{q}^{-1}\left(\widetilde{\mathcal{Y}}_{\mathbf{m}(k)}^{\dagger}\right)$, and $\varphi_{0}, \zeta_{0}$ are restrictions of $\varphi$, $\zeta$, respectively. Again, the squares in the diagrams are both cartesian. 
By (3.3.1) we have a decomposition

$$
\tilde{\mathcal{Y}}_{\mathbf{m}(k)}^{\dagger}=\coprod_{\mathbf{I} \in \mathcal{I} \bullet(\mathbf{m}(k))} \tilde{\mathcal{Y}}_{\mathbf{I}}
$$

into irreducible components, where

$$
\mathcal{I}^{\bullet}(\mathbf{m}(k))=\left\{\mathbf{I} \in \mathcal{I}(\mathbf{m}(k)) \mid I_{i}=I_{i}^{\circ}(1 \leq i \leq r-2), I_{r-1}, I_{r} \subset I_{r-1}^{\circ}\right\}
$$

Let $\psi_{\mathbf{I}}$ be the restriction of the map $\tilde{\mathcal{Y}}_{\mathbf{m}(k)}^{\dagger} \rightarrow \mathcal{Y}_{\mathbf{m}(k)}^{0}$ on $\widetilde{\mathcal{Y}}_{\mathbf{I}}$ for each $\mathbf{I} \in \mathcal{I}^{\bullet}(\mathbf{m}(k))$ as in 1.3. Under the notation in 1.3, the map $\psi_{\mathbf{I}}$ factors through $\widehat{\mathcal{Y}}_{\mathbf{I}}$ as $\psi_{\mathbf{I}}=\eta_{\mathbf{I}} \circ \xi_{\mathbf{I}}$

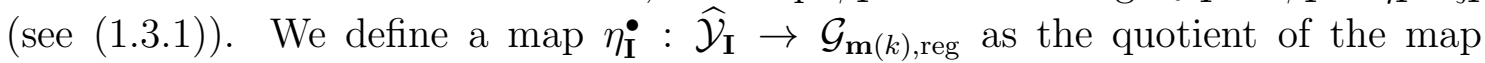
$(g,(t, \mathbf{v})) \mapsto\left(g t g^{-1}, g \mathbf{v},\left(g \bar{M}_{I_{i}}\right)_{1 \leq i \leq r-2}\right)$. Thus $\eta_{\mathbf{I}}: \widehat{\mathcal{Y}}_{\mathbf{I}} \rightarrow \mathcal{Y}_{\mathbf{m}(k)}^{0}$ factors through $\mathcal{G}_{\mathbf{m}(k), \text { reg }}$ as $\eta_{\mathbf{I}}=\zeta_{0} \circ \eta_{\mathbf{I}}^{\bullet}$.

The variety $\widetilde{\mathcal{Y}}_{\mathbf{m}^{\prime}(k)}^{\prime \dagger}$ is also decomposed into irreducible components as in (8.2.2), by using the parameter set $\mathcal{I}^{\bullet}\left(\mathbf{m}^{\prime}(k)\right)$. For $\mathbf{I}^{\prime} \in \mathcal{I}^{\bullet}\left(\mathbf{m}^{\prime}(k)\right)$, the varieties $\widehat{\mathcal{Y}}_{\mathbf{I}^{\prime}}^{\prime}$ are defined with respect to $\mathcal{X}_{\mathbf{m}^{\prime}}^{\prime}$. Note that teh set $\mathcal{I}^{\bullet}\left(\mathbf{m}^{\prime}(k)\right)$ is in bijection with the set $\mathcal{I}^{\bullet}(\mathbf{m}(k))$ under the correspondence $\mathbf{I}^{\prime} \leftrightarrow \mathbf{I}=\left(I_{1}^{\circ}, \mathbf{I}^{\prime}\right)$. Now the commutative diagram (8.2.1) impies the following commutative diagram for each $\mathbf{I} \in \mathcal{I}^{\bullet}(\mathbf{m}(k))$

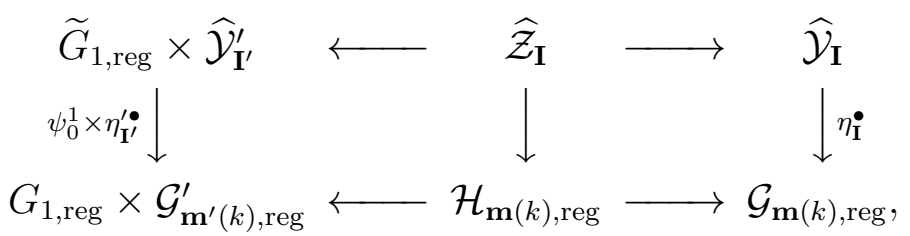

where $\widehat{\mathcal{Z}}_{\mathbf{I}}$ is the fibre product of $\mathcal{H}_{\mathbf{m}(k) \text {,reg }}$ and $\mathcal{Y}_{\mathbf{I}}$ over $\mathcal{G}_{\mathbf{m}(k) \text {,reg. }}$. The both squares in the diagram are cartesian squares. By 1.3 , we know that $\eta_{\mathbf{I}}: \widehat{\mathcal{Y}}_{\mathbf{I}} \rightarrow \mathcal{Y}_{\mathbf{m}(k)}^{0}$ is a finite Galois covering with group $\mathcal{W}_{\mathbf{I}}$. Here we note that

(8.2.4) $\eta_{\mathbf{I}}^{\bullet}: \widehat{\mathcal{Y}}_{\mathbf{I}} \rightarrow \mathcal{G}_{\mathbf{m}(k), \text { reg }}$ is a finite Galois covering with group $\mathcal{W}_{\mathbf{I}}$. In particular, the restriction of $\zeta_{0}$ gives an isomorphism $\mathcal{G}_{\mathbf{m}(k), \text { reg }} \underset{\rightarrow}{\rightarrow} \mathcal{Y}_{\mathbf{m}(k)}^{0}$.

In fact, this is clear in the case where $r=2$, since $\mathcal{G}_{\mathbf{m}(k) \text {,reg }}=\mathcal{Y}_{\mathbf{m}(k)}^{0}$. Assume that $r \geq 3$, and that (8.2.4) holds for $r-1$. By induction, $\eta_{\mathbf{I}^{\prime}}^{\prime}: \widehat{\mathcal{Y}}_{\mathbf{I}^{\prime}}^{\prime} \rightarrow \widehat{\mathcal{G}}_{\mathbf{m}^{\prime}(k) \text {,reg }}^{\prime}$ is a finite Galois covering with group $\mathcal{W}_{\mathbf{I}^{\prime}}^{\prime}$. Then $\psi_{0}^{1} \times \eta_{\mathbf{I}^{\prime}}^{\prime \bullet}$ is a finite Galois covering with group $S_{m_{1}} \times \mathcal{W}_{\mathbf{I}^{\prime}}^{\prime}=\mathcal{W}_{\mathbf{I}}$. Since both squares in the diagram (8.2.3) are cartesians, $\eta_{\mathbf{I}}^{\bullet}$ is a finite Galois covering with group $\mathcal{W}_{\mathbf{I}}$. Since $\eta_{\mathbf{I}}$ is also a finite Galois covering with group $\mathcal{W}_{\mathbf{I}}$, we conclude that $\mathcal{G}_{\mathbf{m}(k), \text { reg }} \simeq \mathcal{Y}_{\mathbf{m}(k)}^{0}$. This proves (8.2.4).

Since $\eta_{\mathbf{I}}$ is a finite Galois covering with group $\mathcal{W}_{\mathbf{I}} \simeq \mathcal{W}_{\mathbf{m}(k)}$, by (1.5.3) we have

$$
\left(\eta_{\mathbf{I}}\right) ! \overline{\mathbf{Q}}_{l} \simeq \bigoplus_{\rho_{0} \in \mathcal{W}_{\mathbf{m}(k)}^{\wedge}} \rho_{0} \otimes \mathcal{L}_{\rho_{0}}
$$


where $\mathcal{L}_{\rho_{0}}$ is a simple local system on $\mathcal{Y}_{\mathbf{m}(k)}^{0}$. We regard $\mathcal{L}_{\rho_{0}}$ as a simple local system on $\mathcal{G}_{\mathbf{m}(k) \text {,reg }}$ under the isomorphism $\mathcal{G}_{\mathbf{m}(k), \text { reg }} \simeq \mathcal{Y}_{\mathbf{m}(k)}^{0}$.

Now take $\rho \in\left(\mathcal{W}_{\mathbf{m}}^{\natural}\right)^{\wedge}$. There exist a unique integer $k$ and $\rho_{0} \in \mathcal{W}_{\mathbf{m}(k)}^{\wedge}$ such that $\rho=V_{\rho_{0}}^{\natural}$. Then we have $K_{\rho}=\operatorname{IC}\left(\mathcal{X}_{\mathbf{m}(k)}, \mathcal{L}_{\rho_{0}}\right)\left[d_{\mathbf{m}(k)}\right]$. Put $A_{\rho}=\operatorname{IC}\left(\overline{\mathcal{G}}_{\mathbf{m}(k)}, \mathcal{L}_{\rho_{0}}\right)\left[d_{\mathbf{m}(k)}\right]$. $A_{\rho}$ is an $H$-equivariant simple perverse sheaf on $\overline{\mathcal{G}}_{\mathbf{m}(k)}$, and we regard it as a perverse sheaf on $\mathcal{G}_{\mathrm{m}}$ by extension by zero.

We show the following fact.

Proposition 8.3. Assume that $\mathcal{X}_{\mathbf{m}}$ is of exotic type.

(i) $\varphi_{!} \overline{\mathbf{Q}}_{l}\left[d_{\mathbf{m}}\right]$ is a semisimple perverse sheaf on $\mathcal{G}_{\mathbf{m}}$ equipped with $\mathcal{W}_{\mathbf{m}}^{\natural}$-action, and is decomposed as

$$
\varphi_{!} \overline{\mathbf{Q}}_{l}\left[d_{\mathbf{m}}\right] \simeq \bigoplus_{\rho \in\left(\mathcal{W}_{\mathbf{m}}^{\natural}\right)^{\wedge}} \rho \otimes A_{\rho}
$$

(ii) $\zeta_{!} A_{\rho} \simeq K_{\rho}$.

Proof. We prove the proposition by induction on $r$. In the case where $r=2$, $\mathbf{m}=\left(m_{1}, m_{2}\right)=(n, 0)$ since $\mathbf{m} \in \mathcal{Q}_{n, r}^{0}$. Thus $\mathcal{G}_{\mathbf{m}}=\mathcal{X}_{\mathbf{m}}=\mathcal{X}$ and $\varphi=\pi: \widetilde{\mathcal{X}} \rightarrow \mathcal{X}$. Moreover, $\zeta$ is the identity map, and $\mathcal{W}_{\mathrm{m}}^{\natural}=W_{n}$. Hence the proposition follows from [SS1, Theorem 4.2]. Assume that $r \geq 3$, and that the proposition holds for $r-1$. We know, under the notation of 2.6, that

$$
\pi_{!}^{1} \overline{\mathbf{Q}}_{l}\left[\operatorname{dim} G_{1}\right] \simeq \bigoplus_{\rho_{1} \in S_{m_{1}}^{\wedge}} \rho_{1} \otimes \operatorname{IC}\left(G_{1}, \mathcal{L}_{\rho_{1}}\right)\left[\operatorname{dim} G_{1}\right]
$$

On the other hand, by applying the induction hypothesis to $\mathcal{X}_{\mathbf{m}^{\prime}}^{\prime}$, we have

$$
\varphi_{!}^{\prime} \overline{\mathbf{Q}}_{l}\left[d_{\mathbf{m}^{\prime}}\right] \simeq \bigoplus_{\rho^{\prime} \in\left(\mathcal{W}_{\mathbf{m}^{\prime}}^{\natural}\right)^{\wedge}} \rho^{\prime} \otimes A_{\rho^{\prime}}
$$

where $A_{\rho^{\prime}}$ is a simple perverse sheaf on $\mathcal{G}_{\mathbf{m}^{\prime}}^{\prime}$ defined similarly to $A_{\rho}$. By applying the argument in 2.6, one can find a unique $H$-equivariant simple perverse sheaf $\widetilde{A}_{\rho}$ on $\mathcal{G}_{\mathbf{m}}$ such that

$$
q^{*} \widetilde{A}_{\rho}\left[\beta_{2}\right] \simeq \sigma^{*}\left(K_{\rho_{1}} \otimes A_{\rho^{\prime}}\right)\left[\beta_{1}\right]
$$

where $\beta_{1}=\operatorname{dim} H+(r-2) m_{1}, \beta_{2}=\operatorname{dim} H_{0}$, and $K_{\rho_{1}}=\operatorname{IC}\left(G_{1}, \mathcal{L}_{\rho_{1}}\right)\left[\operatorname{dim} G_{1}\right]$. It follows from the discussion in 8.2 that $\widetilde{A}_{\rho}$ actually coincides with $A_{\rho}$. Put $K=$ $\varphi_{!} \overline{\mathbf{Q}}_{l}\left[d_{\mathbf{m}}\right], K^{\prime}=\varphi_{!}^{\prime} \overline{\mathbf{Q}}_{l}\left[d_{\mathbf{m}^{\prime}}\right]$ and $K^{1}=\left(\pi^{1}\right) ! \overline{\mathbf{Q}}_{l}\left[\operatorname{dim} G_{1}\right]$. Since both squares in (8.1.1) are cartesian, we have

$$
q^{*} K\left[\beta_{2}\right] \simeq \sigma^{*}\left(K_{1} \otimes K^{\prime}\right)\left[\beta_{1}\right]
$$

Combining (8.3.1), (8.3.2) and (8.3.3), we obtain 


$$
K \simeq \bigoplus_{\rho \in\left(\mathcal{W}_{\mathbf{m}}^{\natural}\right)^{\wedge}} \rho \otimes A_{\rho} .
$$

By this decomposition, $K=\varphi_{!} \overline{\mathbf{Q}}_{l}\left[d_{\mathbf{m}}\right]$ is regarded as a complex with $\mathcal{W}_{\mathbf{m}}^{\natural}$-action. This proves (i).

Next we show (ii). Since $\zeta$ is proper, $\zeta_{!} A_{\rho}$ is a semisimple complex on $\mathcal{X}_{\mathbf{m}}$. By (i), $K=\varphi_{!} \overline{\mathbf{Q}}_{l}\left[d_{\mathbf{m}}\right]$ is a semisimple perverse sheaf. Since $\zeta_{!} K \simeq\left(\pi^{(\mathbf{m})}\right)_{!} \overline{\mathbf{Q}}_{l}\left[d_{\mathbf{m}}\right]$ is a semisimple perverse sheaf, it follows that $\zeta_{!} A_{\rho}$ is a semisimple perverse sheaf. By (8.2.4) we have $\left.\left.\zeta ! A_{\rho}\right|_{\mathcal{Y}_{\mathbf{m}(k)}^{0}} \simeq K_{\rho}\right|_{\mathcal{Y}_{\mathbf{m}(k)}^{0}}$. Hence $\zeta_{!} A_{\rho}$ contains $K_{\rho}$ as a direct summand. By applying $\zeta$ ! to the formula in (i), we have

$$
\left(\pi^{(\mathbf{m})}\right)_{!} \overline{\mathbf{Q}}_{l}\left[d_{\mathbf{m}}\right] \simeq \bigoplus_{\rho \in\left(\mathcal{W}_{\mathbf{m}}^{\natural}\right)^{\wedge}} \rho \otimes \zeta_{!} A_{\rho}
$$

By Theorem 3.2, we have

$$
\left(\pi^{(\mathbf{m})}\right)_{!} \overline{\mathbf{Q}}_{l}\left[d_{\mathbf{m}}\right] \simeq \bigoplus_{\rho \in\left(\mathcal{W}_{\mathbf{m}}^{\natural}\right)^{\wedge}} \rho \otimes K_{\rho} .
$$

By comparing these two formulas, we obtain (ii). The proposition is proved.

8.4. For each $\mathbf{m} \in \mathcal{Q}_{n, r}^{0}$, put $\mathcal{G}_{\mathbf{m} \text { uni }}=\zeta^{-1}\left(\mathcal{X}_{\mathbf{m}, \text { uni }}\right)$. Then the map $\pi_{1}^{(\mathbf{m})}$ is decomposed as

$$
\pi_{1}^{(\mathbf{m})}: \widetilde{\mathcal{X}}_{\mathbf{m}, \text { uni }} \stackrel{\varphi_{1}}{\longrightarrow} \mathcal{G}_{\mathbf{m}, \text { uni }} \stackrel{\zeta_{1}}{\longrightarrow} \mathcal{X}_{\mathbf{m}, \text { uni }},
$$

where $\varphi_{1}, \zeta_{1}$ are restrictions of $\varphi, \zeta$, respectively. Note that $\varphi_{1}$ is surjective. Put $\mathcal{H}_{\mathrm{m} \text { uni }}=q^{-1}\left(\mathcal{G}_{\mathbf{m} \text {,uni }}\right)$. The inclusion map $\mathcal{G}_{\mathbf{m} \text {,uni }} \hookrightarrow \mathcal{G}_{\mathbf{m}}$ is compatible with the diagram (8.1.1), namely we have a commutative diagram

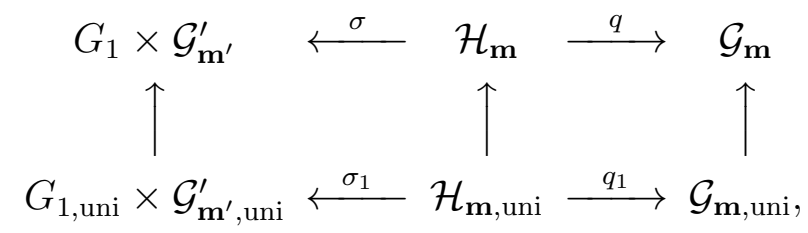

where $\sigma_{1}, q_{1}$ are restrictions of $\sigma, q$ respectively, and vertical maps are natural inclusions. A simialr property as (8.1.2) still holds for $q_{1}, \sigma_{1}$, and both squares are cartesian squares.

For each $\boldsymbol{\lambda} \in \mathcal{P}(\mathbf{m}(k))$, we define a subset $\mathcal{G}_{\boldsymbol{\lambda}}$ of $\mathcal{G}_{\mathbf{m}}$ uni inductively as follows; Write $\boldsymbol{\lambda}=\left(\lambda^{(1)}, \boldsymbol{\lambda}^{\prime}\right)$ with $\boldsymbol{\lambda}^{\prime} \in \mathcal{P}_{n-m_{1}, r-1}$. Assume that $G_{2}^{\theta}$-stable subset $\mathcal{G}_{\boldsymbol{\lambda}^{\prime}}^{\prime}$ of $\mathcal{G}_{\mathbf{m}^{\prime} \text {,uni }}^{\prime}$ was defined. Let $\mathcal{O}_{\lambda^{(1)}}$ be the $G_{1^{-}}$orbit in $\left(G_{1}\right)_{\text {uni }}$ corresponding to $\lambda^{(1)}$, and put $Z=\mathcal{O}_{\lambda^{(1)}} \times \mathcal{G}_{\lambda^{\prime}}^{\prime}$. Then $\sigma_{1}^{-1}(Z)$ is an $H_{0^{-}}$stable subset of $\mathcal{H}_{\mathbf{m} \text {,uni }}$, and $q_{1} \circ \sigma_{1}^{-1}(Z)$ coincides with the quotient of $\sigma_{1}^{-1}(Z)$ by $H_{0}$. We define $\mathcal{G}_{\boldsymbol{\lambda}}$ by $\mathcal{G}_{\boldsymbol{\lambda}}=q_{1} \circ \sigma_{1}^{-1}(Z)$. In the case where $r=3, \mathbf{m}=\left(m_{1}, m_{2}, 0\right)$ and $\mathbf{m}(k)=\left(m_{1}, k, k^{\prime}\right)$ with $k+k^{\prime}=m_{2}$. $\mathbf{m}^{\prime}=\left(m_{2}, 0\right)$ and $\mathcal{G}_{\mathbf{m}^{\prime} \text {,uni }}^{\prime}=\mathcal{X}_{\text {uni }}^{\prime}$, where $\mathcal{X}^{\prime}$ is the exotic symmetric space for $r=2$ associated to $G_{2}$. In this case, $\boldsymbol{\lambda}^{\prime}=\left(\lambda^{(2)}, \lambda^{(3)}\right) \in \mathcal{P}_{m_{2}, 2}$, and we take $\mathcal{G}_{\boldsymbol{\lambda}^{\prime}}^{\prime}$ as the 
$G_{2}^{\theta}$-orbit in $\mathcal{X}_{\text {uni }}^{\prime}$ corresponding to $\boldsymbol{\lambda}^{\prime}$. Thus $\mathcal{G}_{\boldsymbol{\lambda}}$ is well-defined, and $\mathcal{G}_{\boldsymbol{\lambda}}$ turns out to be a smooth irreducible $H$-stable subvariety of $\mathcal{G}_{\mathbf{m}}$,uni. By induction on $r$, we show the following formulas.

$$
\operatorname{dim} \mathcal{G}_{\boldsymbol{\lambda}}=2 n^{2}-2 n-2 n(\boldsymbol{\lambda})-2 n\left(\lambda^{(r-1)}+\lambda^{(r)}\right)+\sum_{i=1}^{r-1}(r-i+1)\left|\lambda^{(i)}\right| .
$$

In fact by (8.1.2), we have

$$
\operatorname{dim} \mathcal{G}_{\boldsymbol{\lambda}}=\operatorname{dim} \mathcal{O}_{\lambda^{(1)}}+\operatorname{dim} \mathcal{G}_{\boldsymbol{\lambda}^{\prime}}^{\prime}+\left(\operatorname{dim} H+(r-2) m_{1}\right)-\operatorname{dim} H_{0}
$$

By applying the induction hypothesis for $\boldsymbol{\lambda}^{\prime}$, (with $n^{\prime}=n-m_{1}$ ), we have

$$
\operatorname{dim} \mathcal{G}_{\boldsymbol{\lambda}^{\prime}}^{\prime}=2 n^{\prime 2}-2 n^{\prime}-2 n\left(\boldsymbol{\lambda}^{\prime}\right)-2 n\left(\lambda^{(r-1)}+\lambda^{(r)}\right)+\sum_{i=2}^{r-1}(r-i+1)\left|\lambda^{(i)}\right|,
$$

and $\operatorname{dim} \mathcal{O}_{\lambda^{(1)}}=m_{1}^{2}-m_{1}-2 n\left(\lambda^{(1)}\right)$. Moreover $\operatorname{dim} H=2 n^{2}+n, \operatorname{dim} H_{0}=m_{1}^{2}+$ $2 n^{\prime 2}+n^{\prime}$. Substituting these formulas into (8.4.3), we obtain (8.4.2).

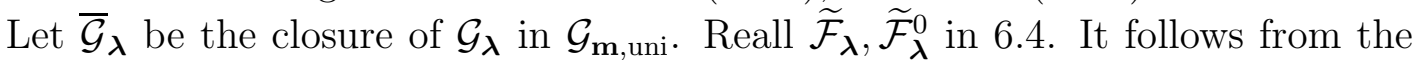
construction, $\widetilde{\mathcal{F}}_{\boldsymbol{\lambda}}$ is a closed subset of $\mathcal{G}_{\mathbf{m} \text {,uni }}$. We have the following lemma.

Lemma 8.5. Assume that $\mathcal{X}_{\mathbf{m}}$ is of exotic type.

(i) $\overline{\mathcal{G}}_{\boldsymbol{\lambda}}$ coincides with $\widetilde{\mathcal{F}}_{\boldsymbol{\lambda}}$. In particular, $\widetilde{\mathcal{F}}_{\boldsymbol{\lambda}}^{0}$ is an open dense subset of $\overline{\mathcal{G}}_{\boldsymbol{\lambda}}$.

(ii) $\zeta_{1}\left(\overline{\mathcal{G}}_{\boldsymbol{\lambda}}\right)=\bar{X}_{\boldsymbol{\lambda}}$, and $\zeta_{1}^{-1}\left(X_{\boldsymbol{\lambda}}\right)=\widetilde{\mathcal{F}}_{\boldsymbol{\lambda}}^{0}$. Hence the restriction of $\zeta_{1}$ on $\zeta_{1}^{-1}\left(X_{\boldsymbol{\lambda}}\right)$ gives an isomorphism $\zeta_{1}^{-1}\left(X_{\boldsymbol{\lambda}}\right) \stackrel{\sim}{\rightarrow} X_{\boldsymbol{\lambda}}$.

Proof. By induction on $r$, one can verify that $\widetilde{\mathcal{F}}_{\lambda}^{0} \subset \mathcal{G}_{\boldsymbol{\lambda}}$. Hence $\widetilde{\mathcal{F}}_{\boldsymbol{\lambda}} \subset \overline{\mathcal{G}}_{\boldsymbol{\lambda}}$. By Lemma 6.6 and by $(8.4 .2), \operatorname{dim} \widetilde{\mathcal{F}}_{\boldsymbol{\lambda}}=\operatorname{dim} \overline{\mathcal{G}}_{\boldsymbol{\lambda}}$. Since both are irredcuible closed subsets of $\mathcal{G}_{\text {m,uni }}$, we have $\widetilde{\mathcal{F}}_{\boldsymbol{\lambda}}=\overline{\mathcal{G}}_{\boldsymbol{\lambda}}$. This proves (i). Then the restriction of $\zeta_{1}$ on $\overline{\mathcal{G}}_{\boldsymbol{\lambda}}$ coincides with the map $\pi_{\boldsymbol{\lambda}}: \widetilde{\mathcal{F}}_{\boldsymbol{\lambda}} \rightarrow \bar{X}_{\boldsymbol{\lambda}}$. Hence (ii) follows from Proposition 6.7.

8.6 Recall the set $\widetilde{\mathcal{P}}(\mathbf{m})$ in (6.8.1) for each $\mathbf{m} \in \mathcal{Q}_{n, r}^{0}$. It is well-known that $\left(\mathcal{W}_{\mathbf{m}}^{\natural}\right)^{\wedge}$ is naturally parametrized by the set $\widetilde{\mathcal{P}}(\mathbf{m})$. We denote by $\rho_{\boldsymbol{\lambda}}$ the irreducible representation of $\mathcal{W}_{\mathbf{m}}^{\natural}$ corresponding to $\boldsymbol{\lambda} \in \widetilde{\mathcal{P}}(\mathbf{m})$.

For each $\boldsymbol{\lambda} \in \widetilde{\mathcal{P}}(\mathbf{m})$, let $V(\boldsymbol{\lambda})$ be the irreducible representation of $W_{n, r}$ obtained from $\rho_{\boldsymbol{\lambda}}$ as in 7.13. Then we have

$$
W_{n, r}^{\wedge} \simeq \coprod_{\mathbf{m} \in \mathcal{Q}_{n, r}^{0}} \widetilde{\mathcal{P}}(\mathbf{m})
$$

We have the following refinements of Theorem 7.12 and Corollary 7.14.

Theorem 8.7. Assume that $\mathcal{X}_{\mathbf{m} \text {,uni }}$ is of exotic type with $\mathbf{m} \in \mathcal{Q}_{n, r}^{0}$. 
(i) Let $L_{\rho}$ be as in Theorem 7.12. Assume that $\rho=\rho_{\boldsymbol{\lambda}} \in\left(\mathcal{W}_{\mathbf{m}}^{\natural}\right)^{\wedge}$ for $\boldsymbol{\lambda} \in \widetilde{\mathcal{P}}(\mathbf{m})$. Then we have

$$
L_{\rho} \simeq \operatorname{IC}\left(\bar{X}_{\boldsymbol{\lambda}}, \overline{\mathbf{Q}}_{l}\right)\left[\operatorname{dim} X_{\boldsymbol{\lambda}}\right]
$$

(ii) (Springer correspondence for $\mathcal{W}_{\mathbf{m}}^{\natural}$ )

$$
\left(\pi_{1}^{(\mathbf{m})}\right)_{!} \overline{\mathbf{Q}}_{l}\left[d_{\mathbf{m}}^{\prime}\right] \simeq \underset{\boldsymbol{\lambda} \in \widetilde{\mathcal{P}}(\mathbf{m})}{\bigoplus} \rho_{\boldsymbol{\lambda}} \otimes \operatorname{IC}\left(\bar{X}_{\boldsymbol{\lambda}}, \overline{\mathbf{Q}}_{l}\right)\left[\operatorname{dim} X_{\boldsymbol{\lambda}}\right]
$$

(iii) (Springer correspondence for $W_{n, r}$ )

$$
\left(\bar{\pi}_{\mathbf{m}, 1}\right)_{!} \overline{\mathbf{Q}}_{l}\left[d_{\mathbf{m}}^{\prime}\right] \simeq \bigoplus_{\boldsymbol{\lambda} \in \widetilde{\mathcal{P}}(\mathbf{m})} V(\boldsymbol{\lambda}) \otimes \operatorname{IC}\left(\bar{X}_{\boldsymbol{\lambda}}, \overline{\mathbf{Q}}_{l}\right)\left[\operatorname{dim} X_{\boldsymbol{\lambda}}\right]
$$

Proof. By Proposition 8.3, we know that $\zeta_{!} A_{\rho}=K_{\rho}$. Hence by the base change theorem, $\left.\left(\zeta_{1}\right)_{!}\left(\left.A_{\rho}\right|_{\mathcal{G}_{\mathbf{m} \text {,uni }}}\right) \simeq K_{\rho}\right|_{\mathcal{X}_{\mathbf{m} \text {,uni }}}$. For $\boldsymbol{\lambda} \in \mathcal{P}(\mathbf{m}(k))$, we define a simple perverse sheaf $B_{\boldsymbol{\lambda}}$ on $\mathcal{G}_{\mathrm{m} \text {,uni }}$ inductively as follows; in the case where $r=2$, put $B_{\boldsymbol{\lambda}}=$ $\operatorname{IC}\left(\overline{\mathcal{O}}_{\boldsymbol{\lambda}}, \overline{\mathbf{Q}}_{l}\right)\left[\operatorname{dim} \mathcal{O}_{\boldsymbol{\lambda}}\right]$, where $\mathcal{O}_{\boldsymbol{\lambda}}$ is the $H$-orbit in $G_{\text {uni }}^{\iota \theta} \times V$ corresponding to $\boldsymbol{\lambda}$. In general for $\boldsymbol{\lambda} \in \mathcal{P}(\mathbf{m}(k))$, put $\boldsymbol{\lambda}=\left(\lambda^{(1)}, \boldsymbol{\lambda}^{\prime}\right)$ with $\left|\lambda^{(1)}\right|=m_{1}, \boldsymbol{\lambda}^{\prime} \in \mathcal{P}\left(\mathbf{m}^{\prime}(k)\right)$. We assume that a simple perverse sheaf $B_{\boldsymbol{\lambda}^{\prime}}$ on $\mathcal{G}_{\mathbf{m}^{\prime} \text {,uni }}^{\prime}$ is already defined. By a similar construction as $\widetilde{A}_{\rho}$ in the proof of Proposition 8.3, there exists a unique simple perverse sheaf $B_{\boldsymbol{\lambda}}$ on $\mathcal{G}_{\mathbf{m} \text {,uni }}$ satisfying the relation

$$
q_{1}^{*} B_{\boldsymbol{\lambda}}\left[\beta_{2}\right] \simeq \sigma_{1}^{*}\left(K_{\lambda^{(1)}} \otimes B_{\boldsymbol{\lambda}^{\prime}}\right)\left[\beta_{1}\right]
$$

where $K_{\lambda^{(1)}}=\operatorname{IC}\left(\overline{\mathcal{O}}_{\lambda^{(1)}}, \overline{\mathbf{Q}}_{l}\right)\left[\operatorname{dim} \mathcal{O}_{\lambda^{(1)}}\right]$ for the $G L_{m_{1}}$-orbit $\mathcal{O}_{\lambda^{(1)}}$ in $\left(G L_{m_{1}}\right)$ uni corresponding to $\lambda^{(1)}$, and $\beta_{1}, \beta_{2}$ are as in (8.3.3). Assume that $\rho=\rho_{\boldsymbol{\lambda}}$. By comparing the construction of $\widetilde{A}_{\rho}$ and of $B_{\boldsymbol{\lambda}}$, and by using induction hypothesis, we see that the restriction of $A_{\rho}$ on $\mathcal{G}_{\mathbf{m}}$,uni coincides with $B_{\boldsymbol{\lambda}}$, up to shift. Moreover, by induction, one can show that the restriction of $B_{\boldsymbol{\lambda}}$ on $\mathcal{G}_{\boldsymbol{\lambda}}$ is a constant sheaf $\overline{\mathbf{Q}}_{l}$. In particular, $\operatorname{supp} B_{\boldsymbol{\lambda}}=\overline{\mathcal{G}}_{\boldsymbol{\lambda}}$. Then by Lemma 8.5 , the support of $\left(\zeta_{1}\right)_{!} B_{\boldsymbol{\lambda}}$ coincides with $\bar{X}_{\boldsymbol{\lambda}}$. By (7.12.2), we know that the restriction of $K_{\rho}$ on $\mathcal{X}_{\mathbf{m} \text {,uni }}$ is a simple perverse sheaf $L_{\rho}$, up to shift. We show that

$$
L_{\rho} \simeq \operatorname{IC}\left(\bar{X}_{\boldsymbol{\lambda}}, \overline{\mathbf{Q}}_{l}\right)\left[\operatorname{dim} X_{\boldsymbol{\lambda}}\right]
$$

For this it is enough to see that $\left.L_{\rho}\right|_{X_{\boldsymbol{\lambda}}}$ is a constant sheaf $\overline{\mathbf{Q}}_{l}$. But by Lemma 8.5 (ii), $\zeta_{1}^{-1}\left(X_{\boldsymbol{\lambda}}\right)=\widetilde{\mathcal{F}}_{\boldsymbol{\lambda}}^{0} \subset \mathcal{G}_{\boldsymbol{\lambda}}$, and the restriction of $B_{\boldsymbol{\lambda}}$ on $\mathcal{G}_{\boldsymbol{\lambda}}$ is the constant sheaf $\overline{\mathbf{Q}}_{l}$, up to shift. Since $\zeta_{1}^{-1}\left(X_{\boldsymbol{\lambda}}\right) \stackrel{\sim}{\rightarrow} X_{\boldsymbol{\lambda}}$ by Lemma 8.5 (ii), we see that $\left.\left(\zeta_{1}\right)_{!} B_{\boldsymbol{\lambda}}\right|_{X_{\boldsymbol{\lambda}}}$ coincides with $\overline{\mathbf{Q}}_{l}$ up to shift. Thus (8.7.1) holds, and (i) follows. (ii) and (iii) then follows from Theorem 7.12 and Corollary 7.14. The theorem is proved.

8.8. We now assume that $\mathcal{X}$ is of enhanced type with $\mathbf{m} \in \mathcal{Q}_{n, r}$. We define a variety $\mathcal{G}_{\mathbf{m}}$ by 


$$
\begin{array}{r}
\mathcal{G}_{\mathbf{m}}=\left\{\left(x, \mathbf{v},\left(W_{i}\right)_{1 \leq i \leq r-1}\right) \mid(x, \mathbf{v}) \in \mathcal{X},\left(W_{i}\right): \text { partial flag in } V\right. \\
\left.\operatorname{dim} W_{i}=p_{i}, x\left(W_{i}\right)=W_{i}, v_{i} \in W_{i}(1 \leq i \leq r-1)\right\}
\end{array}
$$

Let $\zeta: \mathcal{G}_{\mathbf{m}} \rightarrow \mathcal{X}$ be the projection to the first and second factors. Then the map $\pi^{(\mathbf{m})}: \widetilde{\mathcal{X}}_{\mathbf{m}} \rightarrow \mathcal{X}_{\mathbf{m}}$ is decomposed as $\pi^{(\mathbf{m})}=\zeta \circ \varphi$, where $\varphi: \widetilde{\mathcal{X}}_{\mathbf{m}} \rightarrow \mathcal{G}_{\mathbf{m}}, \zeta: \mathcal{G}_{\mathbf{m}} \rightarrow \mathcal{X}_{\mathbf{m}}$ are defined similarly to the exotic case. Here $\varphi$ and $\zeta$ are surjective maps. Since $\operatorname{dim} \widetilde{\mathcal{X}}_{\mathbf{m}}=\operatorname{dim} \mathcal{X}_{\mathbf{m}}$ by Lemma 4.2, we have $\operatorname{dim} \mathcal{G}_{\mathbf{m}}=\operatorname{dim} \mathcal{X}_{\mathbf{m}}$. Put $V_{0}=M_{p_{1}}, \bar{V}_{0}=$ $V / M_{p_{1}}$. We define a variety $\mathcal{H}_{\mathbf{m}}$ by

$$
\begin{array}{r}
\mathcal{H}_{\mathbf{m}}=\left\{\left(x, \mathbf{v},\left(W_{i}\right), \phi_{1}, \phi_{2}\right) \mid\left(x, \mathbf{v},\left(W_{i}\right)\right) \in \mathcal{G}_{\mathbf{m}}\right. \\
\left.\phi_{1}: W_{1} \underset{\rightarrow}{\sim} V_{0}, \phi_{2}: V / W_{1} \stackrel{\sim}{\rightarrow} \bar{V}_{0}\right\}
\end{array}
$$

We also define a variety $\widetilde{\mathcal{Z}}_{\mathbf{m}}$ by

$$
\begin{aligned}
\widetilde{\mathcal{Z}}_{\mathbf{m}}=\left\{\left(x, \mathbf{v}, g B^{\theta}, \phi_{1}, \phi_{2}\right)\right. & \mid\left(x, \mathbf{v}, g B^{\theta}\right) \in \widetilde{\mathcal{X}}_{\mathbf{m}}, \\
\phi_{1}: g\left(M_{p_{1}}\right) & \left.\stackrel{\sim}{\rightarrow} V_{0}, \phi_{2}: V / g\left(M_{p_{1}}\right) \stackrel{\sim}{\rightarrow} \bar{V}_{0}\right\} .
\end{aligned}
$$

Assume that $r \geq 3$, and let $\mathbf{m}^{\prime}=\left(m_{2}, \ldots, m_{r}\right)$ for $\mathbf{m}=\left(m_{1}, \ldots, m_{r}\right)$. Let $G_{1}=$ $G L\left(V_{0}\right)$ and $G_{2}=G L\left(\bar{V}_{0}\right) \times G L\left(\bar{V}_{0}\right)$ with the permutation action $\theta: G_{2} \rightarrow G_{2}$. Then $\widetilde{G}_{1}, \widetilde{\mathcal{X}}_{\mathbf{m}^{\prime}}^{\prime}, \mathcal{X}_{\mathbf{m}^{\prime}}^{\prime}$ etc. are defined similarly to the exotic case. Note that $\mathcal{X}_{\mathbf{m}^{\prime}}^{\prime}$ is a closed subvareity of $G_{2}^{\iota \theta} \times \bar{V}_{0}^{r-2} \simeq G L\left(\bar{V}_{0}\right) \times \bar{V}_{0}^{r-2}$. Then a similar commutative diagram as (8.1.1) holds also for the enhanced case. We use the same notaion as in (8.1.1). One can check that the maps $q, \sigma$ satisfy similar properties as in (8.1.2), namely we have

(8.8.1) $q$ is a principal bundle with fibre isomorphic to $H_{0}$, and $\sigma$ is a locally trivial fibration with smooth fibre of dimension $\operatorname{dim} H+(r-1) m_{1}$. (It should be noticed about the difference on the dimension of the fibre. Also note in this case, $H_{0} \simeq G L\left(V_{0}\right) \times G L\left(\bar{V}_{0}\right)$, and $H \simeq G L(V)$.)

8.9. For $\mathbf{m} \in \mathcal{Q}_{n, r}$, put $\tilde{\mathcal{Y}}_{\mathbf{m}}^{\dagger}=\left(\psi^{(\mathbf{m})}\right)^{-1}\left(\mathcal{Y}_{\mathbf{m}}^{0}\right)$. Then under the notation in 4.1, $\widetilde{\mathcal{Y}}_{\mathbf{m}}^{\dagger}$ coinicdes with $\widetilde{\mathcal{Y}}_{\mathbf{I}}$ for $\mathbf{I}=\mathbf{I}^{\bullet}$ in 3.3. In particular, $\psi_{\mathbf{I}}: \widetilde{\mathcal{Y}}_{\mathbf{I}} \rightarrow \mathcal{Y}_{\mathbf{m}}^{0}$ is a finite Galois covering with group $\mathcal{W}_{\mathbf{I}}=\mathcal{W}_{n}^{\natural}$. $\widetilde{\mathcal{Y}}_{\mathbf{m}}^{\dagger}$ is an open dense subsete of $\widetilde{\mathcal{Y}}_{\mathbf{m}}$. Put $\mathcal{G}_{\mathbf{m} \text {,reg }}=\zeta^{-1}\left(\mathcal{Y}_{\mathbf{m}}^{0}\right) \cdot \mathcal{G}_{\mathbf{m} \text {,reg }}$ is an open subset of $\mathcal{G}_{\mathbf{m}}$. The varieties $\mathcal{Y}_{\mathbf{m}^{\prime}}^{\prime 0}, \widetilde{\mathcal{Y}}_{\mathbf{m}^{\prime}}^{\prime \dagger}$ and $\mathcal{G}_{\mathbf{m}^{\prime} \text {,reg }}^{\prime}$ are defined similarly. As in the exotic case, we have the following commutative diagram. 


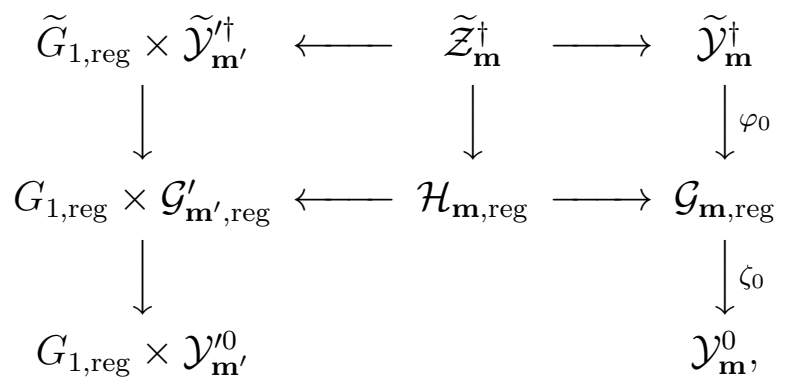

By using (8.9.1) and by induction on $r$, one can show that $\varphi_{0}: \widetilde{\mathcal{Y}}_{\mathbf{m}}^{\dagger} \rightarrow \mathcal{G}_{\mathbf{m} \text {,reg }}$ is a finite Galois covering with group $\mathcal{W}_{\mathbf{m}}^{\natural}$. It follows that

(8.9.2) $\zeta_{0}$ gives an isomorphism $\mathcal{G}_{\mathbf{m}, \text { reg }} \underset{\rightarrow}{\rightarrow} \mathcal{Y}_{\mathbf{m}}^{0}$.

For each $\rho \in \mathcal{W}_{\mathbf{m}}^{\natural}$, we consider $K_{\rho}=\operatorname{IC}\left(\mathcal{X}_{\mathbf{m}}, \mathcal{L}_{\rho}\right)\left[d_{\mathbf{m}}\right]$, where $\mathcal{L}_{\rho}$ is a simple local system on $\mathcal{Y}_{\mathbf{m}}^{0}$ obtained from the Galois covering $\mathcal{W}_{\mathbf{m}}^{\natural}$. We regard $\mathcal{L}_{\rho}$ as a local system on $\mathcal{G}_{\mathbf{m} \text {,reg }}$ under the isomorphism $\mathcal{G}_{\mathbf{m} \text {,reg }} \simeq \mathcal{Y}_{\mathbf{m}}^{0}$. Put $A_{\rho}=\operatorname{IC}\left(\mathcal{G}_{\mathbf{m}, \text { reg }}, \mathcal{L}_{\rho}\right)\left[d_{\mathbf{m}}\right]$.

The following result can be proved in a similar way as Proposition 8.3, by making use of Theorem 4.5, instead of Theorem 3.2.

Proposition 8.10. Assume that $\mathcal{X}_{\mathbf{m}}$ is of enhanced type.

(i) $\varphi_{!} \overline{\mathbf{Q}}_{l}\left[d_{\mathbf{m}}\right]$ is a semisimple perverse sheaf on $\mathcal{G}_{\mathbf{m}}$ equipped with $\mathcal{W}_{\mathbf{m}}^{\natural}$-action, and is decomposed as

$$
\varphi_{!} \overline{\mathbf{Q}}_{l}\left[d_{\mathbf{m}}\right] \simeq \bigoplus_{\rho \in\left(\mathcal{W}_{\mathbf{m}}^{\natural}\right)^{\wedge}} \rho \otimes A_{\rho}
$$

(ii) $\zeta_{!} A_{\rho} \simeq K_{\rho}$.

8.11. For each $\mathbf{m} \in \mathcal{Q}_{n, r}$, put $\mathcal{G}_{\mathbf{m} \text {,uni }}=\zeta^{-1}\left(\mathcal{X}_{\mathbf{m}, \text { uni }}\right)$. Then the map $\pi_{1}^{(\mathbf{m})}$ is decomposed as $\pi_{1}^{(\mathbf{m})}=\zeta_{1} \circ \varphi_{1}$ as in the exotic case (see 8.4), where $\varphi_{1}: \widetilde{\mathcal{X}}_{\mathbf{m} \text {,uni }} \rightarrow$ $\mathcal{G}_{\mathrm{m} \text {,uni }}$ and $\zeta_{1}: \mathcal{G}_{\mathbf{m} \text {,uni }} \rightarrow \mathcal{X}_{\mathrm{m} \text {,uni }}$ are restrictions of $\varphi, \zeta$. Note that $\varphi_{1}$ is surjective. Put $\mathcal{H}_{\mathrm{m} \text { uni }}=q^{-1}\left(\mathcal{G}_{\mathbf{m} \text {,uni }}\right)$. Then we have a similar commutative diagram as (8.4.1).

For $\boldsymbol{\lambda} \in \mathcal{P}(\mathbf{m})$, we define a subset $\mathcal{G}_{\boldsymbol{\lambda}}$ of $\mathcal{G}_{\mathbf{m}}$,uni inductively, by applying the discussion in 8.4 for the exotic case. Note that in the case where $r=2, \mathbf{m}=\left(m_{1}, m_{2}\right)$ and $\mathbf{m}^{\prime}=\left(m_{2}\right) . \mathcal{G}_{\mathbf{m}^{\prime}}=\mathcal{X}_{\text {uni }}^{\prime}$. In this case, we take $\mathcal{G}_{\boldsymbol{\lambda}^{\prime}}^{\prime}$ as the $G_{2}^{\theta}$-orbit in $\mathcal{X}_{\text {uni }}^{\prime}$ corresponding to $\boldsymbol{\lambda}^{\prime}=\lambda^{(2)}$. Thus $\mathcal{G}_{\boldsymbol{\lambda}}$ is defined, and $\mathcal{G}_{\boldsymbol{\lambda}}$ is a smooth irreducible $H$-stable subvariety of $\mathcal{G}_{\mathbf{m} \text {,uni }}$.

As in the exotic case (see (8.4.2)), one can compute the dimension of $\mathcal{G}_{\boldsymbol{\lambda}}$ by making use of (8.8.1). We have

$$
\operatorname{dim} \mathcal{G}_{\boldsymbol{\lambda}}=n^{2}-n-2 n(\boldsymbol{\lambda})+\sum_{i=1}^{r-1}(r-i)\left|\lambda^{(i)}\right|
$$


Recall the definition of $\widetilde{\mathcal{F}}_{\boldsymbol{\lambda}}$ and $\widetilde{\mathcal{F}}_{\boldsymbol{\lambda}}^{0}$ (in the enhanced case) in 6.18. The following lemma can be proved in a similar way as Lemma 8.5. Note that $\operatorname{dim} \widetilde{\mathcal{F}}_{\boldsymbol{\lambda}}=\operatorname{dim} \overline{\mathcal{G}}_{\boldsymbol{\lambda}}$ by Proposition 5.4 and Proposition 6.19, together with (8.11.1). We use Proposition 6.19 instead of Proposition 6.7.

Lemma 8.12. Assume that $\mathcal{G}_{\boldsymbol{\lambda}}$ is of enhanced type. A similar statement as in Lemma 8.5 holds also for $\mathcal{G}_{\lambda}$.

We can now obtain a refinement of Theorem 7.12 in the enhanced case. The proof is similar to the proof of Theorem 8.7. Note that Thorem 8.13 (ii) is obtained by $\mathrm{Li}$ [Li, Th. 3.2.6] by a different method.

Theorem 8.13. Assume that $\mathcal{X}_{\mathbf{m} \text {,uni }}$ is of enhanced type.

(i) Let $L_{\rho}$ be as in Theorem 7.12. Assume that $\rho=\rho_{\boldsymbol{\lambda}} \in\left(\mathcal{W}_{\mathbf{m}}^{\natural}\right)^{\wedge}$ for $\boldsymbol{\lambda} \in \mathcal{P}(\mathbf{m})$. Then we have

$$
L_{\rho} \simeq \mathrm{IC}\left(\bar{X}_{\boldsymbol{\lambda}}, \overline{\mathbf{Q}}_{l}\right)\left[\operatorname{dim} X_{\boldsymbol{\lambda}}\right]
$$

(ii) (Springer correspondence for $\mathcal{W}_{\mathbf{m}}^{\natural}$ )

$$
\left(\pi_{1}^{(\mathbf{m})}\right)_{!} \overline{\mathbf{Q}}_{l}\left[d_{\mathbf{m}}^{\prime}\right] \simeq \bigoplus_{\boldsymbol{\lambda} \in \mathcal{P}(\mathbf{m})} \rho_{\boldsymbol{\lambda}} \otimes \operatorname{IC}\left(\bar{X}_{\boldsymbol{\lambda}}, \overline{\mathbf{Q}}_{l}\right)\left[\operatorname{dim} X_{\boldsymbol{\lambda}}\right]
$$

8.14. Assume that $\mathcal{X}_{\mathbf{m}}$ is of exotic type or of enhanced type, under the setting in 8.1. For each $z \in \mathcal{X}_{\mathbf{m}}$, we consider the (small) Springer fibre $\mathcal{B}_{z}^{(\mathbf{m})}=\left(\pi^{(\mathbf{m})}\right)^{-1}(z)$. In the exotic case, we also consider the Springer fibre $\mathcal{B}_{z}=\pi^{-1}(z)$ (see 6.20). We have $\mathcal{B}_{z}^{(\mathbf{m})} \subset \mathcal{B}_{z}$. The cohomology group $H^{i}\left(\mathcal{B}_{z}^{(\mathbf{m})}, \overline{\mathbf{Q}}_{l}\right)$ has a structure of $\mathcal{W}_{\mathbf{m}}^{\natural}$-module. In turn, $H^{i}\left(\mathcal{B}_{z}, \overline{\mathbf{Q}}_{l}\right)$ has a structure of $W_{n, r}$-module. Put $d_{\boldsymbol{\lambda}}=\left(\operatorname{dim} \mathcal{X}_{\mathbf{m}, \text { uni }}-\operatorname{dim} X_{\boldsymbol{\lambda}}\right) / 2$ for $\boldsymbol{\lambda} \in \widetilde{\mathcal{P}}(\mathbf{m})$ in the exotic case, and for $\boldsymbol{\lambda} \in \mathcal{P}(\mathbf{m})$ in the enhanced case. Explicitly, we have

$$
d_{\boldsymbol{\lambda}}= \begin{cases}\left(m_{r-1}-k\right)+n(\boldsymbol{\lambda})+n\left(\lambda^{(r-1)}+\lambda^{(r)}\right) & : \text { exotic case, } \boldsymbol{\lambda} \in \mathcal{P}(\mathbf{m}(k)), \\ n(\boldsymbol{\lambda}) & : \text { enhanced case, } \boldsymbol{\lambda} \in \mathcal{P}(\mathbf{m})\end{cases}
$$

As shown in the example in $5.18, \operatorname{dim} \mathcal{B}_{z}^{(\mathbf{m})}$ is not constant for $z \in X_{\boldsymbol{\lambda}}$. We show the following lemma.

Lemma 8.15. Assume that $\mathcal{X}_{\mathbf{m}}$ is of exotic type or of enhanced type.

(i) For any $z \in X_{\boldsymbol{\lambda}}, \operatorname{dim} \mathcal{B}_{z}^{(\mathbf{m})} \geq d_{\boldsymbol{\lambda}}$. The set $z \in X_{\boldsymbol{\lambda}}$ such that $\operatorname{dim} \mathcal{B}_{z}^{(\mathbf{m})}=d_{\boldsymbol{\lambda}}$ forms an open dense subset of $X_{\boldsymbol{\lambda}}$.

(ii) For any $z \in X_{\boldsymbol{\lambda}}, H^{2 d_{\boldsymbol{\lambda}}}\left(\mathcal{B}_{z}^{(\mathbf{m})}, \overline{\mathbf{Q}}_{l}\right)$ contains an irreducible $\mathcal{W}_{\mathbf{m}}^{\natural}$-module $\rho_{\boldsymbol{\lambda}}$.

Proof. First we show (ii). Assume that $\mathcal{X}_{\mathbf{m}}$ is of exotic type. For any $z \in \mathcal{X}_{\mathbf{m} \text {,uni, }}$ Theorem 8.7 (ii) implies that

$$
H^{i}\left(\mathcal{B}_{z}^{(\mathbf{m})}, \overline{\mathbf{Q}}_{l}\right) \simeq \bigoplus_{\boldsymbol{\mu} \in \widetilde{\mathcal{P}}(\mathbf{m})} \rho_{\boldsymbol{\mu}} \otimes \mathcal{H}_{z}^{i-d_{\mathbf{m}}^{\prime}+\operatorname{dim} X_{\boldsymbol{\mu}}}\left(\operatorname{IC}\left(\bar{X}_{\boldsymbol{\mu}}, \overline{\mathbf{Q}}_{l}\right)\right)
$$


as $\mathcal{W}_{\mathbf{m}}^{\natural}$-modules. Assume that $z \in X_{\boldsymbol{\lambda}}$, and put $i=2 d_{\boldsymbol{\lambda}}$. Since $\mathcal{H}_{z}^{0}\left(\operatorname{IC}\left(\bar{X}_{\boldsymbol{\lambda}}, \overline{\mathbf{Q}}_{l}\right)\right)=$ $\overline{\mathbf{Q}}_{l}, H^{2 d_{\boldsymbol{\lambda}}}\left(\mathcal{B}_{z}^{(\mathbf{m})}, \overline{\mathbf{Q}}_{l}\right)$ contains $\rho_{\boldsymbol{\lambda}}$. This proves (ii). The enhanced case is proved in a similar way by using Theorem 8.13 (ii).

(ii) implies, in particular, $\operatorname{dim} \mathcal{B}_{z}^{(\mathbf{m})} \geq d_{\boldsymbol{\lambda}}$. Put $d=\operatorname{dim}\left(\pi^{(\mathbf{m})}\right)^{-1}\left(X_{\boldsymbol{\lambda}}\right)-\operatorname{dim} X_{\boldsymbol{\lambda}}$. Let $X(d)$ be as in 6.20. Then $X(d) \cap X_{\boldsymbol{\lambda}}$ is open dense in $X_{\boldsymbol{\lambda}}$. Hence $\operatorname{dim} X_{\boldsymbol{\lambda}} \leq$ $\operatorname{dim} X(d)$. By Lemma 6.21 (iii), we have

$$
\operatorname{dim} \mathcal{B}_{z}^{(\mathbf{m})} \leq \frac{1}{2}\left(\operatorname{dim} \mathcal{X}_{\mathbf{m}, \text { uni }}-\operatorname{dim} X(d)\right) \leq \frac{1}{2}\left(\operatorname{dim} \mathcal{X}_{\mathbf{m}, \text { uni }}-\operatorname{dim} X_{\boldsymbol{\lambda}}\right)=d_{\boldsymbol{\lambda}}
$$

for any $z \in X_{\boldsymbol{\lambda}} \cap X(d)$. Hence $\operatorname{dim} \mathcal{B}_{z}^{(\mathbf{m})}=d_{\boldsymbol{\lambda}}$ and $d=d_{\boldsymbol{\lambda}}$. This proves (i).

We show the following result. In the enhanced case, a similar result was proved in [Li, Cor. 3.2.9] for the Borel-Moore homology.

Proposition 8.16. Assume that $\mathcal{X}_{\mathrm{m}}$ is of exotic type or of enhanced type. Take $z \in X_{\boldsymbol{\lambda}}$ such that $\operatorname{dim} \mathcal{B}_{z}^{(\mathbf{m})}=d_{\boldsymbol{\lambda}}$.

(i) $H^{2 d_{\lambda}}\left(\mathcal{B}_{z}^{(\mathbf{m})}, \overline{\mathbf{Q}}_{l}\right) \simeq \rho_{\boldsymbol{\lambda}}$ as $\mathcal{W}_{\mathbf{m}}^{\natural}$-modules.

(ii) Assume that $\mathcal{X}_{\mathbf{m}}$ is of exotic type. Then $\operatorname{dim} \mathcal{B}_{z}=d_{\boldsymbol{\lambda}}$, and $H^{2 d_{\boldsymbol{\lambda}}}\left(\mathcal{B}_{z}, \overline{\mathbf{Q}}_{l}\right) \simeq$ $V(\boldsymbol{\lambda})$ as $W_{n, r}$-modules.

Proof. We prove (i) by induction on $r$. Assume that $r=2$. In the exotic case, (i) holds by [SS1, Remarks 5.5 (ii)]. A similar method also works for the enhanced case, since the number of $H$-orbits in $\mathcal{X}_{\text {uni }}$ is finite. Assume that $r \geq 3$, and that (i) holds for $r-1$. We consider the diagram as in (8.1.1) restricted to the unipotent varieties as discussed in 8.4. Put $\boldsymbol{\lambda}=\left(\lambda^{(1)}, \boldsymbol{\lambda}^{\prime}\right)$ as in 8.4. By (8.14.1), we have $d_{\boldsymbol{\lambda}}=d_{\boldsymbol{\lambda}^{\prime}}+d_{\lambda^{(1)}}$, where $d_{\boldsymbol{\lambda}^{\prime}}$ is defined similarly to $d_{\boldsymbol{\lambda}}$, and $d_{\lambda^{(1)}}=\left(\operatorname{dim} G_{1, \text { uni }}-\operatorname{dim} \mathcal{O}_{\lambda^{(1)}}\right) / 2$. Take $z \in X_{\boldsymbol{\lambda}}$ such that $\operatorname{dim} \mathcal{B}_{z}^{(\mathbf{m})}=d_{\boldsymbol{\lambda}}$. By Lemma 8.5 and Lemma 8.12, $\zeta_{1}$ gives an isomorphism $\zeta_{1}^{-1}\left(X_{\boldsymbol{\lambda}}\right) \rightarrow X_{\boldsymbol{\lambda}}$. Hence there exists a unique $z_{*} \in \zeta_{1}^{-1}\left(X_{\boldsymbol{\lambda}}\right)$ such that $\zeta_{1}\left(z_{*}\right)=z$. Then by using the diagram (8.1.1), one can find $\left(x_{1}, z^{\prime}\right) \in \mathcal{O}_{\lambda^{(1)}} \times X_{\lambda^{\prime}}^{\prime}$ and $z_{*}^{\prime}=\left(\zeta_{1}^{\prime}\right)^{-1}\left(z^{\prime}\right)$ such that $\sigma_{1}^{-1}\left(x_{1}, z_{*}^{\prime}\right)=q_{1}^{-1}\left(z_{*}\right)$. Here $z^{\prime} \in X_{\lambda^{\prime}}^{\prime}$ satisfies the condition that $\operatorname{dim} \mathcal{B}_{z^{\prime}}^{\left(\mathbf{m}^{\prime}\right)}=d_{\boldsymbol{\lambda}^{\prime}}$. By using the isomorphism $\left(\zeta_{1}^{\prime}\right)^{-1}\left(X_{\boldsymbol{\lambda}^{\prime}}^{\prime}\right) \stackrel{\sim}{\rightarrow} X_{\boldsymbol{\lambda}^{\prime}}^{\prime}$, we have

$$
H^{2 d_{\lambda^{\prime}}}\left(\mathcal{B}_{z^{\prime}}^{\left(\mathbf{m}^{\prime}\right)}, \overline{\mathbf{Q}}_{l}\right) \simeq\left(R^{2 d_{\lambda^{\prime}}}\left(\pi_{1}^{\prime}\right) ! \overline{\mathbf{Q}}_{l}\right)_{z^{\prime}} \simeq\left(R^{2 d_{\lambda^{\prime}}}\left(\varphi_{1}^{\prime}\right)_{!} \overline{\mathbf{Q}}_{l}\right)_{z_{*}^{\prime}},
$$

where $\varphi_{1}^{\prime}$ is the restriction of $\varphi^{\prime}$. Similarly we have $H^{2 d_{\lambda}}\left(\mathcal{B}_{z}^{(\mathbf{m})}, \overline{\mathbf{Q}}_{l}\right) \simeq\left(R^{2 d_{\lambda}}\left(\varphi_{1}\right) ! \overline{\mathbf{Q}}_{l}\right)_{z_{*}}$ by using $\zeta^{-1}\left(X_{\boldsymbol{\lambda}}\right) \stackrel{\sim}{\rightarrow} X_{\boldsymbol{\lambda}}$. Let $\xi$ be an element contained in $\sigma_{1}^{-1}\left(x_{1}, z_{*}^{\prime}\right)=q_{1}^{-1}\left(z_{*}\right)$. By (8.1.1), we have

$$
\left(R^{2 d_{\lambda^{(1)}}}\left(\pi_{1}^{1}\right)_{!} \overline{\mathbf{Q}}_{l}\right)_{x_{1}} \otimes\left(R^{2 d_{\lambda^{\prime}}}\left(\varphi_{1}^{\prime}\right)_{!} \overline{\mathbf{Q}}_{l}\right)_{z_{*}^{\prime}} \simeq\left(R^{2 d_{\lambda}}\left(\widetilde{\varphi}_{1}\right)_{!} \overline{\mathbf{Q}}_{l}\right)_{\xi} \simeq\left(R^{2 d_{\lambda}}\left(\varphi_{1}\right)_{!} \overline{\mathbf{Q}}_{l}\right)_{z_{*}},
$$

where $\pi_{1}^{1}, \widetilde{\varphi}_{1}$ are restrictions of $\pi^{1}, \widetilde{\varphi}$, respectively. By induction, we know that $\operatorname{dim} H^{2 d_{\lambda^{\prime}}}\left(\mathcal{B}_{z^{\prime}}^{\left(\mathbf{m}^{\prime}\right)}, \overline{\mathbf{Q}}_{l}\right)=\operatorname{dim} \rho_{\boldsymbol{\lambda}^{\prime}}$. It is well-konwn that $\operatorname{dim} H^{2 d_{\lambda^{(1)}}}\left(\mathcal{B}_{x_{1}}, \overline{\mathbf{Q}}_{l}\right)=\operatorname{dim} \rho_{\lambda^{(1)}}$. Hence $\operatorname{dim} H^{2 d_{\boldsymbol{\lambda}}}\left(\mathcal{B}_{z}^{(\mathbf{m})}, \overline{\mathbf{Q}}_{l}\right)=\operatorname{dim} \rho_{\lambda^{(1)}}+\operatorname{dim} \rho_{\boldsymbol{\lambda}^{\prime}}=\operatorname{dim} \rho_{\boldsymbol{\lambda}}$. On the other hand, by Lemma $8.15, H^{2 d_{\boldsymbol{\lambda}}}\left(\mathcal{B}_{z}^{(\mathbf{m})}, \overline{\mathbf{Q}}_{l}\right)$ contains $\rho_{\boldsymbol{\lambda}}$. Thus $H^{2 d_{\boldsymbol{\lambda}}}\left(\mathcal{B}_{z}^{(\mathbf{m})}, \overline{\mathbf{Q}}_{l}\right) \simeq \rho_{\boldsymbol{\lambda}}$. (i) is proved. 
Next we show (ii). Assume that $\mathcal{X}_{\mathbf{m}}$ is of exotic type and $z \in X_{\boldsymbol{\lambda}}$ such that $\operatorname{dim} \mathcal{B}_{z}^{(\mathbf{m})}=d_{\boldsymbol{\lambda}}$. We consider the decomposition (8.15.1) in the case where $z \in X_{\boldsymbol{\lambda}}$. By Theorem 8.7 (iii), we have a similar decomposition

$$
H^{i}\left(\mathcal{B}_{z}, \overline{\mathbf{Q}}_{l}\right) \simeq \bigoplus_{\boldsymbol{\mu} \in \widetilde{\mathcal{P}}(\mathbf{m})} V(\boldsymbol{\mu}) \otimes \mathcal{H}_{z}^{i-d_{\mathbf{m}}^{\prime}+\operatorname{dim} X_{\mu}}\left(\mathrm{IC}\left(\bar{X}_{\boldsymbol{\mu}}, \overline{\mathbf{Q}}_{l}\right)\right)
$$

(8.15.1) shows that $\mathcal{H}_{z}^{i-d_{\mathbf{m}}^{\prime}+\operatorname{dim} X_{\boldsymbol{\mu}}}\left(\operatorname{IC}\left(\bar{X}_{\boldsymbol{\mu}}, \overline{\mathbf{Q}}_{l}\right)\right)=0$ for any choice of $i>2 d_{\boldsymbol{\lambda}}$ and of $\boldsymbol{\mu} \in \widetilde{\mathcal{P}}(\mathbf{m})$. This implies, by (8.16.1), that $H^{i}\left(\mathcal{B}_{z}, \overline{\mathbf{Q}}_{l}\right)=0$ for any $i>2 d_{\boldsymbol{\lambda}}$. Since $\mathcal{B}_{z}^{(\mathbf{m})} \subset \mathcal{B}_{z}$, we conclude that $\operatorname{dim} \mathcal{B}_{z}=\operatorname{dim} \mathcal{B}_{z}^{(\mathbf{m})}=d_{\boldsymbol{\lambda}}$. Now assume that $i=2 d_{\boldsymbol{\lambda}}$. By (i) and (8.15.1), we see that $\mathcal{H}_{z}^{2 d_{\boldsymbol{\lambda}}-d_{\mathbf{m}}^{\prime}+\operatorname{dim} X_{\boldsymbol{\mu}}}\left(\operatorname{IC}\left(\bar{X}_{\boldsymbol{\mu}}, \overline{\mathbf{Q}}_{l}\right)\right)=0$ for any $\boldsymbol{\mu} \neq \boldsymbol{\lambda}$. Hence by (8.16.1), $H^{2 d_{\boldsymbol{\lambda}}}\left(\mathcal{B}_{z}, \overline{\mathbf{Q}}_{l}\right) \simeq V(\boldsymbol{\lambda})$. The proposition is proved.

\section{REFERENCES}

[AH] P. Achar and A. Henderson; Orbit closures in the enhanced nilpotent cone, Adv. in Math. 219 (2008), 27-62. Corrigendum, ibid. 228 (2011), 2984-2988.

[FGT] M. Finkelberg, V. Ginzburg and R. Travkin; Mirabolic affine Grassmannian and chartacter sheaves, Selecta Math. 14 (2009), 607-628.

[K] S. Kato; An exotic Deligne-Langlands correspondence for symplectic groups, Duke Math. J. 148 (2009), 306-371.

[Li] Y. Li; A class of perverse sheaves on framed representation varieties of the Jordan quiver, preprint.

[Lu] G. Lusztig; Intersection cohomology complexes on a reductive group, Invent. Math.75 (1984), 205-272.

[SS1] T. Shoji and K. Sorlin; Exotic symmetric space over a finite field, I, Transformation Groups, 18, (2013) 877-929.

[SS2] T. Shoji and K. Sorlin; Exotic symmetric space over a finite field, III, to appear in Transformation Groups.

[T] R. Travkin; Mirabolic Robinson-Schensted correspondence, Selecta Math. 14 (2009), 727-758.

\section{T. Shoji}

Department of Mathematics, Tongji University

2139 Siping Road, Shanghai 200092, P.R. China

E-mail: shoji@tongji.edu.cn 